\title{
Proceedings of the British Thoracic Society
}

\section{Working for healthier lungs}

\author{
The 1989 winter meeting of the British Thoracic Society was held on 7 and 8 December at \\ Kensington Town Hall, London
}

\begin{abstract}
Computed tomographic scans of the lung help distinguish Pneumocystis carinii pneumonia from Kaposi's sarcoma
\end{abstract}

RF MILLER, AB MILLAR, P SHAW, SJG SEMPLE Department of Medicine, UCMSM, Middlesex Hospital, and Department of Radiology, University College Hospital, London The differentiation between Pneumocystis carini pneumonia (PCP) and Kaposi's sarcoma can be difficult if an HIV positive patien presents with alveolar consolidation and/or interstitial shadowing on the chest radiograph (CXR). We have compared the CXR and thoracic computed tomographic (CT) appearances of $31 \mathrm{HIV}$ positive males during acute respiratory episodes in order to evaluate the role of CT in distinguishing PCP from Kaposi's sarcoma. In group 1 sixteen patients had PCP. Eleven patients in group 2 had Kaposi's sarcoma and in group 3 four patients had both PCP and Kaposi's sarcoma. Both CXR and CT were categorised as being normal $(\mathrm{N})$ or showing one or more of the following abnormalities: interstitial shadowing (INT); alveolar consolidation (ALV); intrapulmonary nodules $\leqslant 1 \mathrm{~cm}$ diameter (NOD); mediastinal and/or hilar lymphadenopathy (LN); pleural effusions (EFF); cystic air spaces (C); and on CT bronchial wall thickening (BT). Results were as follows: Interstitial shadowing, alveolar consolidation and intrapulmonary nodules do not help distinguish Kaposi's sarcoma from PCP. The presence of bronchial wall thickening and cystic air spaces suggest a diagnosis of PCP whereas pleural effusions and lymphadenopathy suggests Kaposi's sarcoma. These abnormalities, which are better demonstrated by CT, help distinguish PCP from intrapulmonary Kaposi's sarcoma.

$N$ INT ALV NODLN EFF C BT

\begin{tabular}{|c|c|c|c|c|c|c|c|}
\hline $\begin{array}{l}\text { Group 1 } \\
(\mathrm{n}=16)\end{array}$ & $\begin{array}{l}\mathrm{CXR} \\
\mathrm{CT}\end{array}$ & $\begin{array}{l}14 \\
14\end{array}$ & $\begin{array}{r}4 \\
10\end{array}$ & $\begin{array}{l}1 \\
7\end{array}$ & $\begin{array}{l}1 \\
5\end{array}$ & $\begin{array}{l}2 \\
2\end{array}$ & $\begin{array}{l}3 \\
6\end{array}$ \\
\hline $\begin{array}{l}\text { Group 2 } \\
(\mathrm{n}=11)\end{array}$ & $\begin{array}{l}\text { CXR } 0 \\
\text { CT } 0\end{array}$ & $\begin{array}{l}9 \\
9\end{array}$ & $\begin{array}{l}5 \\
5\end{array}$ & $\begin{array}{l}5 \\
9\end{array}$ & $\begin{array}{l}2 \\
6\end{array}$ & $\begin{array}{l}7 \\
7\end{array}$ & $\begin{array}{l}0 \\
0\end{array}$ \\
\hline $\begin{array}{l}\text { Group } 3 \\
(n=14)\end{array}$ & $\begin{array}{ll}\text { CXR } & 1 \\
\mathrm{CT} & 0\end{array}$ & $\begin{array}{l}2 \\
2\end{array}$ & $\begin{array}{l}2 \\
2\end{array}$ & $\begin{array}{l}3 \\
3\end{array}$ & $\begin{array}{l}1 \\
1\end{array}$ & $\begin{array}{l}0 \\
0\end{array}$ & $\begin{array}{l}0 \\
1\end{array}$ \\
\hline
\end{tabular}

Pneumocystis carinii: attempted isolation from induced sputum and bronchoalveolar lavage specimens of non-HIV infected individuals using immunofluorescent stains

TR LEIGH, J WIGGINS, J MIDGELEY, N FRANCIS, DC SHANSON, TW EVANS, JV COLlins Westminster and Brompton Hospitals, London Pneumocystis carinii (PC) may be a commensal in non-HIV infected individuals (Pifer. Pediatrics 1978;61:35) and the clinical sig- nificance of detecting PC in induced sputum (IS) and bronchoalveolar lavage fluid (BAL) by highly sensitive immunofluorescent (IF) stains may be questionable (Leigh, BTS, summer 1989). We therefore attempted to isolate PC cysts in IS and BAL specimens of non-HIV infected subjects. IS was obtained following inhalation of $30 \mathrm{mls}$ ultrasonically nebulised (Devilbiss ultraneb 99) hypertonic (3\%) saline. A standard BAL technique was used. All samples were promptly stained using a highly sensitive monoclonal IF stain (Northumbria Biologicals). Twelve IS (from healthy volunteers) and $12 \mathrm{BAL}$ (from patients investigated for possible bronchial carcinoma) samples were studied; cytology confirmed that all specimens sampled the lower respiratory tract. PC was not detected in any samples of IS or BAL. This suggests that, while PC may be a commensal in nonHIV infected individuals, it is not present in sufficient numbers in IS and BAL to be detected by IF. PC detected by this technique is likely to be of clinical significance and studies of asymptomatic HIV individuals are needed.

Continuous positive airways pressure ventilation as an alternative to mechanical ventilation for respiratory failure associated with Pneumocystis carinii pneumonia

RF MILLER, SJG SEMPLE Department of Medicine, UCMSM, Middlesex Hospital, London Despite treatment up to $20 \%$ of patients with Pneumocystis carinii pneumonia (PCP) will develop respiratory failure; in this group of patients mortality is high despite the use of mechanical ventilation. Wachter RM et al. Am Rev Respir Dis 1986;134:891. We have used continuous positive airways pressure (CPAP) ventilation as an alternative to mechanical ventilation in patients with $P C P$ who developed respiratory failure refractory to supplemental oxygen via face mask. Eight HIV positive males with $\mathrm{PCP}$ received CPAP via a tight fitting face mask using a flow generator circuit. On admission patients were treated with intravenous co-trimoxazole (7 patients) or pentamidine (1 patient); admission blood gases breathing room air were $\mathrm{PaO}_{2}$ 32.2-88.9 (mean 58.6) $\mathrm{mm} \mathrm{Hg}$. CPAP was started 1-15 (mean 7) days after admission because of increasing respiratory distress and deteriorating blood gases $\left(\mathrm{PaO}_{2}\right.$ 35.2-78.5 (mean 53.2 ) $\mathrm{mm} \mathrm{Hg}$ while receiving oxygen via face mask at $\mathrm{FiO}_{2}$ 0.6). CPAP was started at $\mathrm{FiO}_{2} 0.6$ and PEEP $5 \mathrm{~cm} \mathrm{H}_{2} \mathrm{O}$ (7 patients) and $10 \mathrm{~cm} \mathrm{H}_{2} \mathrm{O}$ (1 patient). Seven patients showed an improvement in arterial oxygenation $\left(\mathrm{PaO}_{2}\right.$ 50.7-96.4 (mean 78.4) $\left.\mathrm{mm} \mathrm{Hg}\right)$ a mean of four hours after starting CPAP. One patient died one hour after commencing CPAP with deteriorating oxygenation, hypercapnia, and acute right heart failure; at necropsy there was no evidence of pneumothorax. The seven patients made a complete recovery. CPAP was continued for 2-7 (mean 4) days and they were subsequently weaned off it. No major complications (pneumothorax, heart failure) were seen; three patients initially found the CPAP mask claustrophobic but persisted with treatment. Ventilation via mask CPAP has been found to be a useful means of improving oxygenation in patients with PCP who develop respiratory failure and may obviate the need for intubation and mechanical ventilation in such patients.

Aerosol pentamidine as prophylaxis against Pneumocystis carini pneumonia for persons infected with human immunodeficiency virus

ELC ONG, KR NEAL, EM DUNBAR, BK MANDAL Regional Department of Infectious Diseases and Tropical Medicine, Monsall Hospital, Newton Heath, Manchester Pneumocystis carinii pneumonia (PCP) develops in about $80 \%$ of patients with the acquired immunodeficiency syndrome (AIDS); in half of these patients the infection recurs within 12 months. Therefore prophylaxis using inhaled pentamidine aerosol is one method of prevention against subsequent episodes (secondary prophylaxis) or initial episode (primary prophylaxis) for a HIV infected person who has never had PCP. We report our current experience of treating 30 male patients with AIDS or symptomatic HIV infection (median age 36 range 25-56) with weekly nebulised pentamidine isthionate $600 \mathrm{mg}$ dissolved in $6 \mathrm{ml}$ of sterile water using an Antibiotic Tee tube (System 22) and Acorn nebuliser with an Optimist filter (Medic-aid) acting as a baffle delivered at $81 / \mathrm{min}$ pressurised oxygen. This generated aerosol droplets with a mass median aerodynamic diameter of $1.2 \mu \mathrm{m}$ (GSD 2.9) and $89 \%$ were less than $3.9 \mu \mathrm{m}$. On 31 August 198915 patients were receiving pentamidine as secondary prophylaxis. Their mean duration of treatment was 10.8 months (range 4-22). Ten patients who have AIDS and five symptomatic HIV patients whose CD4 + counts were $<200$ / $\mathrm{mm}^{3}$ were treated with this as primary prophylaxis. Their mean duration of treatment was 9.6 months (range 4-16). In both groups there were 22 patients who have been receiving treatment for more than eight months. No new cases or relapses of PCP have occurred in either group of patients. There were three other patients that had PCP previously and received pentamidine as secondary prophylaxis for a mean duration of 10 months (range 9-12) and had since died of other causes. All patients were receiving zidovudine in varying dosages. No serious drug related adverse effects were observed. Our experience suggests that the dose and 
frequency of inhaled pentamidine is a significant factor in determining relapse and initial rate of PCP among HIV infected persons.

Clinical and radiological features of recurrent Pneumocystis carinii pneumonia in patients with the acquired immunodeficiency syndrome (AIDS)

MTA VILLAR, J WIGGINS, P CARVALHO, D SMITH, TW EVANS, JV COLLINS Westminster and Brompton Hospitals, London The classical presentation of first episodes of Pneumocystis carinii pneumonia (PCP) is well recognised. However, atypical features may occur in up to $30 \%$ of patients (De Lorenzo-Chest 1987 ; 91:323) and the features of recurrent PCP have not been described. We compared the clinical and radiological presentation of recurrent episodes (more than six months apart) of microbiologically proved PCP in nine males (mean age (range) 35.5 (29-47) years) with AIDS. All had cough and 8/9 were breathless on both occasions. Atypical presenting symptoms (sputum production, chest pain, sweats, diarrhoea) occurred in $30^{\circ}{ }_{0}$ of patients in each episode but were inconsistent in an individual. In first episodes of PCP, duration of symptoms (means (range) $8.2(1-32)$ and $2.4(1-8)$ weeks respectively) were longer whilst atypical chest radiology (asymmetry, pneumothorax, honeycombing) was less frequent ( 2 of 9 and 5 of 9 respectively). Admission $\mathrm{PaO}_{2}$ (means (range) 11.3 $(9 \cdot 6-17)$ and $10 \cdot 1(8 \cdot 5-12 \cdot 4) \mathrm{kPa}$ respectively) was similar. We conclude that the clinical and radiological features of initial and second episodes of PCP are not comparable in an individual. Reproducibility of these features cannot be used for diagnosis and microbiological confirmation is mandatory when recurrent PCP is suspected.

Pulse mega dose methylprednisolone as adjuvant therapy for the treatment of Pneumocystis carinii pneumonia

RF MILLER, SJG SEMPLE Department of Medicine, UCMSM, Middlesex Hospital, London Initial reports suggested that methylprednisolone in doses of $160-240 \mathrm{mg}$ / day was useful adjunctive therapy in those patients with Pneumocystis carinii pneumonia (PCP) who developed respiratory failure MacFadden DK et al. Lancet 1987;i:1477. Since then the usefulness of this therapy has been questioned Clement M et al. Am Rev Respir Dis 1989;139:A250. In an open prospective study we have used mega dose methylprednisolone in $26 \mathrm{HIV}$ positive males with PCP who became hypoxaemic despite conventional therapy. On admission 20 patients were treated with intravenous cotrimoxazole, five nebulised pentamidine (NP), and one intravenous pentamidine admission $\mathrm{PaO}_{2}$ (breathing room air) 32.696.8 (mean 65.3 ) $\mathrm{mm} \mathrm{Hg}$. Methylprednisolone was begun after 1-18 (mean 4.8) days because of deteriorating $\mathrm{PaO}_{2}$ and worsening dyspnoea/radiographic appearances. One gram of methylprednisolone was given by intravenous infusion over one hour once daily for three days and the five patients receiving NP changed to intravenous co-trimoxazole. Immediately before starting methylpredn- isolone $\mathrm{PaO}_{2}$ (room air) was 29.4-66.9 (mean 45.2) $\mathrm{mm} \mathrm{Hg}$ and 24-36 hours after the first dose of methylprednisolone $\mathrm{PaO}_{2}$ (room air) was $53 \cdot 7-113 \cdot 1 \mathrm{~mm} \mathrm{Hg}$. Twenty three patients $(88 \%)$ responded and subsequently fully recovered; three patients died, one after one dose of methylprednisolone despite an improved $\mathrm{PaO}_{2}$, the two others died after one and two doses of methylprednisolone respectively, with worsening hypoxaemia. No side effects were seen acutely (dyspepsia/gastrointestinal bleeding/sepsis) but one patien had a reactivation of chronic hepatitis $B$ three weeks after the methylprednisolone. Pulse mega dose methylprednisolone appears to be a useful adjuvant therapy for patients with PCP who deteriorate and become hypoxaemic despite conventional therapy.

BTS study of severe community acquired pneumonia in the intensive care unit

MA WOODHEAD for the Pneumonia Standing Subcommittee of the BTS Research Com mittee With the aid of 23 physicians, covering 25 hospitals, a retrospective study of adults admitted in 1987 to an intensive care unit (ITU) with a diagnosis of community acquired pneumonia was undertaken. Fifty nine patients were studied, of whom $34(58 \%)$ were male. The mean age was 54 years (range $19-76)$ with $38(64 \%) \leqslant 65$ and $18(34 \%)$ $\leqslant 44$ years. Twenty three $(39 \%)$ had previously been fit. Median symptom duration before hospital admission was five days (range 1-64). On hospital admission 43 (73\%) had two or more of respiratory rate $\geqslant 30$ diastolic blood pressure $\leqslant 60$ and blood urea $>7 \mathrm{mmol} / 1$. Forty $(64 \%)$ were transferred to the ITU within 48 hours of hospital admission, but eight were admitted to the ITU only after a cardiorespiratory arrest-six $(75 \%)$ of these died. A microbial aetiology was identified in $35(59 \%)$. Pathogens included Streptococcus pneumoniae (11), Legionella pneumophila (7), Haemophilus influenzae (7), Mycoplasma pneumoniae (4) and influenza virus (3); two of which were complicated by infection with Staphylococcus aureus. Dual infection was present in $11(19 \%)$ cases. Fifty two $(88 \%)$ received assisted ventilation (IPPV), $11\left(19 \%\right.$ ) receiving an $\mathrm{Fio}_{2}$ of 1.0 and $28(48 \%)$ positive end expiratory pressure. Twelve of $18(67 \%)$ still receiving IPPV at 14 days survived. Complications were recorded in $43(73 \%)$, but $30(51 \%)$ survived, including all four patients with $M$ pneumoniae and two of three with influenza virus infection. The median duration of hospital stay in survivors was one month. Only $14(24 \%)$ made a complete recovery.

\section{Clinical usefulness of the antineutrophil} cytoplasmic antibody test

CMB HIGgS, MB SAMPSON Chest Unit and Department of Immunology, Royal United Hospital, Bath A recent editorial (Thorax $1989 ; 44: 369-70$ ) states that a positive result in the antineutrophil cytoplasmic antibody test (ANCA) cannot replace the aggressive pursuit of a tissue diagnosis in Wegener's granulomatosis (WG). From a retrospective review of all 44 requests for ANCA in the Bath Health District during March 1987September 1988 , we have assessed the role of
ANCA in diagnosing or excluding WG and the incidence of false negative and positive results. A positive ANCA was defined as the characteristic granular cytoplasmic fluorescence pattern on ethanol fixed neutrophil cytospin preparations present at dilution 1:80. There were nine positive cases: $4 \mathrm{M} 5 \mathrm{~F}$, age 30-81 years, time from onset to diagnosis 2-200 months. All had clinical features entirely consistent with the diagnosis of WG. Eight had a tissue biopsy (4 nasal, 2 renal, 2 lung); in only one (nasal) was the histology regarded as diagnostic of WG, the others being non-specific inflammation or crescentic glomerulonephritis. In three cases the combination of clinical features and histology had established the diagnosis, but in six cases the positive ANCA established the diagnosis and often led to a change in treatment. Of the 35 negative cases, three had previously diagnosed WG now in clinical remission, 32 eventually had other confirmed diagnoses (Churg-Strauss 1, lymphomatoid granulomatosis 1 , connective tissue disorders 6 , glomerulonephritis 6 , others 21 ). There were therefore no false positive or negative results in this study. There were no cases of WG (from diagnostic coding index) diagnosed without ANCA testing. We suggest that in clinical practice, for the diagnosis of WG, the ANCA test, as defined above, could replace tissue biopsy and its reliability is so far excellent.

Progressive lung fibrosis 14 years after childhood BCNU chemotherapy: clinical and physiological features

BR O'DRISCOLL, HR GATTAMANENI, PM TAYLOR, AA WOODCOCK Wythenshawe and Christie Hospitals and University of Manchester BCNU (carmustine) is a cytotoxic drug which is most commonly used to treat malignant gliomas. Like bleomycin and methotrexate, it is a recognised cause of acute lung fibrosis, which may appear up to two years after the completion of therapy. The reported incidence of this complication ranges from $1 \%$ to $20 \%$ (Weiss RB et al, Cancer Treatment Reviews 1981;8:111). Between 1972 and 1976, 30 children (age 2-16 years) with malignant gliomas were treated with surgery, cranial irradiation and BCNU chemotherapy. Thirteen patients died of their brain tumour and two patients died of lung fibrosis within three years of completing therapy. A further four patients have died of "delayed" lung fibrosis 8-13 years after BCNU chemotherapy. Of 11 long term survivors, eight were available for investigation (mean 14 years post treatment, range 13-17). Only two had any respiratory symptoms (breathlessness) and none had finger clubbing or lung crackles. However, all survivors studied had a restrictive spirometric defect (mean FVC $52 \%$ of predicted, range $21-81 \%$ ). The TLCO was reduced (mean $58 \%$ of predicted, range $28-78 \%$ ) but the $\mathrm{Kco}$ was well preserved (mean $117 \%$ of predicted, range $93-137 \%$ ) In one case the FVC had not changed over a five year period, but in three other cases the FVC had declined by 0.1 to 0.8 litres over one to six years. We conclude that "delayed" lung fibrosis is a frequent (possibly universal) consequence of BCNU chemotherapy in childhood. This novel form of lung fibrosis seems to be slowly progressive over a period of at least 15 years. 
Hydrocortisone myopathy in acute severe asthma

CD SHEE Chest Clinic, Medway Hospital, Gillingham, Kent In one hospital over a 15 month period, four out of nine patients ventilated for acute severe asthma developed acute hydrocortisone myopathy. All nine patients had been sedated, and paralysed with vecuronium. All received intravenous salbutamol and aminophylline, nebulised salbutamol, and intravenous hydrocortisone in doses under $1.0 \mathrm{~g} /$ day. Duration of ventilation was 6-19 days in myopathic patients $(3 \mathrm{~F}$, $1 \mathrm{M}$ ) and 2-9 days in the others ( $5 \mathrm{~F}$ ). Affected patients had severe generalised weakness with normal reflexes and sensation. Complete recovery took 1-6 weeks. When myopathic and non-myopathic ventilated asthmatics were compared, there was no clearcut difference with respect to age, sex, types of drug used, serum potassium levels, total dose of vecuronium bromide, or mean daily doses of hydrocortisone. Duration of paralysis was compared to see if neuromuscular blockade might contribute to the development of myopathy. Although myopathic patients had generally been paralysed and ventilated for longer than the unaffected subjects, there was an overlap between the two groups. The main difference between the groups was in total doses of hydrocortisone. Myopathic patients all received $>5.0 \mathrm{~g}$ hydrocortisone (range $5.4-10.2 \mathrm{~g}$ ) and the others $<4.0 \mathrm{~g}$ (range $0.9-3.5 \mathrm{~g}$ ). Hydrocortisone myopathy is probably not an idiosyncratic reaction but rather is dose related. Myopathy can occur on $<1.0 \mathrm{~g} /$ day hydrocortisone, and even with as little as $5.4 \mathrm{~g}$ given over six days.

Methotrexate in steroid dependent asthma: a placebo controlled trial

RJ SHINER, AJ NUNN, F CHUNG, DM GEDDES Brompton Hospital, London Sixty nine steroid dependent asthmatics from 11 specialist centres participated in a randomised, double blind, placebo controlled trial. All patients had been treated with a minimum of $7.5 \mathrm{mg}$, mean (SD) $14 \cdot 17(7 \cdot 10)$, of prednisolone/day, for at least a year in addition to inhaled steroids and bronchodilators. Patients took $15 \mathrm{mg}$ methotrexate or placebo once weekly for 24 weeks. Patients were seen at four weekly intervals by the same physician who reduced the daily prednisolone dose by $2.5 \mathrm{mg}$, depending on satisfactory diary card criteria and spirometry. All other treatment remained unchanged. Prednisolone dosage was compared between the two groups over two four week periods, run in, and 20-24 weeks of treatment. Prednisolone dose was reduced by $50 \%$ in the methotrexate treated group and by $14 \%$ in the placebo group when compared with the run in $(p<0.005)$. Symptom assessment, frequency of night waking, and peak flow measurements did not significantly change in either group during the trial. Abnormal liver function was noted in $12 / 38$, which improved or resolved in seven despite continuation of therapy and persisted or worsened in five. Gastrointestinal side effects were severe in two of 38 and milder and self limiting in six. There were no haematological or pulmonary complications of methotrexate therapy. Methotrexate may have a role in reducing systemic steroid requirement in this group of patients.

(Supported by a grant from the Asthma Research Council.)
Management of recurrent malignant pleural effusion: what are we doing in the UK?

LG MCALPINE, G HULKS, NC THOMSON Department of Respiratory Medicine, Western Infirmary, Glasgow Malignant disease is estimated to account for $25-50 \%$ of cases of pleural effusion. These effusions are often large and recur after simple aspiration. Patients who are reasonably well may benefit from pleurodesis to prevent the need for frequent thoracentesis. Several techniques of pleurodesis have been described and many agents have been subjected to trial yet the approach of practicing clinicians to this problem is not known. We aimed to determine the views of UK clinicians on their approach to the management of recurrent malignant pleural effusion and pleurodesis by means of a postal questionnaire. The 448 clinicians receiving questionnaires consisted of physicians with an interest in respiratory medicine $(n=150)$, general physicians without a respiratory interest $(n=173)$, thoracic surgeons $(n=48)$ and general surgeons $(\mathrm{n}=77)$. There was an overall response rate of $56 \%$. Only 18 general surgeons who saw $>2$ cases/year responded and $44 \%$ of these opted for repeated aspiration alone; further analysis of this group was not performed. Most respiratory physicians $(76 \%)$ would perform pleurodesis in such patients but only $30 \%$ of general physicians would themselves do likewise, with a further $33 \%$ referring the patient to a respiratory specialist and only $15 \%$ managing such patients with repeated aspiration alone. Thoracic surgeons undertake pleurodesis $(81 \%$ ) and this is usually performed under general anaesthetic by both junior and senior staff. Sixty eight percent of respiratory physicians and $90 \%$ of general physicians invariably delegate the task to junior staff. Thoracic surgeons use an intercostal tube drain, usually with suction, while a tube drain was routinely used by only $54 \%$ and $32 \%$ of respiratory and general physicians respectively. The majority of drains are removed within 24 hours. Tetracycline was the agent selected for $2 / 3$ cases of medical pleurodesis while talc was the commonest surgical choice. Local anaesthetic was added to the pleurodesis agent by $52 \%$ and $64 \%$ of respiratory and general physicians respectively. The majority of respondents considered the procedure to be moderately uncomfortable but easily managed with analgesia and to be reasonably effective.

(Supported by an award from the Chest, Heart, and Stroke Association.)

Review of open lung biopsy in 431 patients with diffuse pulmonary lesions

v TSANG, P GOLdSTRAW Brompton Hospital, London Patients with diffuse pulmonary lesions can potentially benefit from the histological information provided by open lung biopsy (OLB), performed via a short inframammary incision (Venn et al. Thorax 1985;40:931). To re-evaluate the diagnostic indications and benefits, the entire series of 431 patients undergoing OLB between July 1979 and August 1989 was reviewed. There were 256 male and 175 female patients, with a mean age of 55 years (range 5 months to 80 years). Twenty four patients $(5.5 \%)$ were immunocompromised (haematological malignancies 17; bone marrow transplants 6; hypogammaglobulinaemia 1). One hundred and twenty five patients $(29 \%)$ were on steroid therapy at the time of operation. OLB provided diagnostic histological material in 409 of the 431 cases $(95 \%)$, cryptogenic fibrosing alveolitis and sarcoidosis being the two most common findings. A diagnosis was obtained in 20 of the 24 immunocompromised cases. An infectious agent was identified in four $(17 \%)$ of the patients (cytomegalovirus 1; Pneumocystis carinii 2; Aspergillus fumigatus 1). Twelve patients $(2 \cdot 7 \%)$ died following OLB, with three $(12.5 \%)$ in the immunocompromised group. All these patients were very ill preoperatively, and only one of the deaths was directly related to the surgical procedure. Twenty one patients $(5 \%)$ suffered complications related to the OLB (wound infection 11; pneumothorax 9; haemothorax 1), with no difference between the immunocompromised and the non-compromised groups. OLB has been shown to be a definitive means of establishing a diagnosis in chronic progressive pulmonary diseases, with minimal risk. In the immunocompromised patients OLB can be performed safely with a significant therapeutic implication.

Mediastinal lymph node size as a predictor of metastatic lung cancer

KM KERR, CG WATHEN, WS WALKER, EW CAMERON, NJ DOUGLAS, D LAMB University of Edinburgh Mediastinal lymph node involvement by tumour has an important bearing on prognosis in lung cancer (Mountain C. Ann Thorac Surg 1977;24:365). Imaging techniques which measure size of mediastinal lymph nodes are often used as a noninvasive method of determining malignancy (eg. Glazer GM et al. AJR 1984;147:1101-5). We have therefore pathologically examined mediastinal lymph nodes in patients with lung cancer as part of a prospective study of the value of mediastinal diagnostic imaging in the detection of mediastinal lymph node metastases. Fifty-six patients with potentially operable histologically proven bronchogenic carcinoma underwent thoracotomy. All accessible mediastinal lymph nodes (N2) were removed at operation and fixed in formalin. The maximum node diameter was measured and each node was then weighed and processed in toto for histological examination. In those which were malignant the proportion of the node replaced by tumour was estimated using a simple point counting technique. Forty-two per cent of the malignant mediastinal nodes $(n=31)$ measured less than $15 \mathrm{~mm}$ (maximum diameter) while $43 \%$ of benign nodes measured $15 \mathrm{~mm}$ or more. Only $23 \%$ of lymph nodes measuring $15 \mathrm{~mm}$ or more were malignant. In this series there is no evidence that malignant mediastinal lymph nodes are larger than benign nodes. These observations help explain the limitations of imaging techniques, which merely record lymph node size in "detecting" mediastinal lymph node metastases.

Survival and prognosis following resection for bronchogenic carcinoma

MA KADRI, JE DUSSEK Brook Hospital and Guy's Hospital, London Between 1980 and 1987495 patients underwent lung resection for primary bronchogenic carcinoma under the care of one surgeon at Guy's Hospital and 
the Brook Hospital, London. The mean age of the patients was 61.9 years and $16.8 \%$ of the population were over the age of 70 . Two hundred and forty-three patients had stage 1 disease, 110 patients stage 2 disease, and 142 patients stage 3 . Lobectomy was performed in 290 patients, pneumonectomy in 197 patients, and wedge resection in 8 patients. Hospital mortality was $5.05 \%$ in all patients, $7 \cdot 11 \%$ following pneumonectomy, $3.79 \%$ after lobectomy; there was no mortality following wedge resection. Overall actuarial survival at one year was $75.8 \%$ and $38.6 \%$ at five years. Actuarial survival for stage 1 disease was $88.5 \%$ at one year and $55.6 \%$ at five years; for stage 2 disease $77.3 \%$ and $34.4 \%$ and for stage 3 disease $57.9 \%$ and $15.4 \%$. With respect to histological type squamous cell carcinoma had the best outlook and oat cell carcinoma the worst. Actuarial survival at five years was $45 \%$ for squamous cell carcinoma, $36.3 \%$ for adenocarcinoma, $31.9 \%$ for dimorphic carcinoma and $21 \%$ for undifferentiated carcinoma. Oat cell carcinoma has a zero four year survival.

Pulmonary resection in children with focal disease due to cystic fibrosis

$P$ HELMS, SK JUSBASCHE, DJ MATTHEW Respiratory Unit, Great Ormond Street Hospital for Sick Children, London Over the period 1975 to 1989 nine cystic fibrosis patients (age range 7 months to $11 \frac{1}{2}$ years) underwent surgical resection of localised bronchiectasis, representing a small proportion $(2.3 \%)$ of the 379 regular clinic attenders. Indications included recurrent severe respiratory exacerbations, failure to thrive and unstable focal disease which was beginning to extend into adjacent areas. During the 14 year period attempts to improve associated collapse/consolidation by bronchoscopy and bronchial lavage were abandoned as no lasting benefit was found. Assessment of the extent of the disease by bronchography was also replaced by radionuclide ventilationperfusion lung scans. There were no perioperative deaths or serious morbidity; the longest period of air leak requiring pleural drainage was 21 days. All patients and or their parents described symptomatic improvements and duration of hospital stay fell from a mean of 36 days (range 1-78) in the year before to 3 days (range $0-10$ ) in the year after surgery. In the five patients old enough to perform reliable spirometry $\%$ predicted FEV 1 and FVC did not change in the year before and after surgery. Lobar resection of localised bronchiectasis can significantly improve the quality of life in severely affected patients with cystic fibrosis; it is a low risk procedure and should be considered in the presence of focal disease with important symptoms.

Diaphragmatic plication for unilateral diaphragmatic paralysis: experience over 10 years and long term follow up

DR GRAHAM, D KAPLAN, CC EVANS, CRK HIND, RJ DONNELLY Regional Adult Cardiothoracic Unit, Liverpool Unilateral paralysis of the

\begin{tabular}{llrl}
\hline & Preoperative & Postoperative & $p$ \\
\hline Dyspnoea (visual analogue scale 0-10) & $7 \cdot 4(0.8)$ & $3.3(0.9)$ & $<0.001$ \\
FVC (sitting) & $2.7(0.7)$ & $3.2(0.5)$ & $<0.001$ \\
FVC (lying) & $1.9(0.5)$ & $2 \cdot 7(0.6)$ & $<0.001$ \\
TLC (sitting) & $4 \cdot 1(1.6)$ & $4.5(1.7)$ & $<0.002$ \\
TLC (lying) & $3.4(0.8)$ & $4.2(1.7)$ & $<0.002$ \\
$\mathrm{PaO}_{2}(\mathrm{kPa})$ & $9 \cdot 4(1.5)$ & $11.5(1.8)$ & $<0.001$ \\
$\mathrm{PaCO}_{2}(\mathrm{kPa})$ & $5 \cdot 1(0.9)$ & $5.1(0.1)$ & $\mathrm{NS}$ \\
\hline
\end{tabular}

diaphragm due to non-malignant disease is an uncommon disorder, which has previously been thought to have benign implications. Some patients, however, experience dyspnoea and orthopnoea accompanied by reduction in pulmonary function tests ( $J$ Thorac Cardiovasc Surg 1985;90:195-8). We have performed unilateral diaphragmatic plication on 17 patients over the last 10 years $(16$ male and one female, mean age 53 (SD 13.8) years range 28-74). Preoperatively each patient was shown to have paradoxical movement of the paralysed diaphragm on sniffing and to have a reduction in FVC and lung volumes. These reductions were greater when the patient was supine. All patients had moderate hypoxaemia (mean 9.45 (SD 1.45) kPa). Plication was performed by imbricating the diaphragm in layers through a thoracotomy incision. After plication all patients showed improvement (table). When six patients were reassessed five or more years after plication (range 5-10 years) all six showed that the improvement which occurred immediately after operation had been maintained. These results suggest that diaphragmatic plication is a safe and effective procedure for adul patients with dyspnoea resulting from unilateral diaphragmatic paralysis. Furthermore, the symptomatic and physiological improvements are maintained long term. Evaluation of silastic endobronchial
stents for bronchial anastomotic
stenosis

V TSANG, M YACOUB, P GOLDSTRAW Brompton Hospital, London Progressive bronchial anastomotic stenosis due to ischaemic dista bronchus after sleeve resection and single lung transplantation (SLT) is a potentially serious complication. In an attempt to avoid traumatic repeated bronchial dilatations and risky rethoracotomy, silastic endobronchial stents were used. There were two male and 1 female patients, with a mean age of 55 years (range 47-65 years). Their original diseases were cryptogenic fibrosing alveolitis, bronchogenic carcinoma, and squamous papilloma. The operations were right SLT, sleeve resection of the right main bronchus, and the left main bronchus respectively. The suture material used for bronchial anastomosis was continuous nonabsorbable monofilament in two and continuous absorbable monofilament in one. Bronchial anastomotic strictures developed within a mean period of 5.3 weeks (range $2-8$ weeks) after the operations with a bronchoscopic appearance of dense florid granulation tissue and concentric fibrosis beneath the bronchial anastomosis. Progressively larger stents $(10-14 \mathrm{~mm}$ diameter) were inserted to maintain the patency of the bronchial anastomosis. Continuous stenting was maintained for a mean period of 16.7 months (range 12-20 months). A simple technique of preparation and inser- tion of the stents was used. There was no complication related to the technique. The endobronchial stents were well tolerated, with clearing of chest infection. Re-expansion of the affected lungs was demonstrated radiographically, associated with improved lung function. The mean $\mathrm{FEV}_{1} / \mathrm{FVC}$ before stenting was $1 \cdot 4 / 2 \cdot 1$ (range $0 \cdot 5-2 \cdot 0 / 1 \cdot 0-2 \cdot 8$ ), and subsequent improvement with one year stenting produced a mean $\mathrm{FEV}_{1} / \mathrm{FVC}$ of $2 \cdot 6$ 3.6 (range 1.8-3.1/2.4-4.9). Our medium term experience with the use of the silastic endobronchial stents as a simple and effective treatment for early bronchial anastomotic strictures is encouraging.

Why are hospital admission and mortality rates for childhood asthma higher in New Zealand than in the United Kingdom?

HR ANDERSON, EA MITCHELL, P FREELING PT WHITE St George's Hospital Medical School, London, and School of Medicine, University of Auckland New Zealand has higher mortality and hospital admission rates for asthma than England and Wales. To determine the reasons for this, available and special survey data from the Auckland Region of New Zealand were compared with those from the South West Thames Region of England. Asthma mortality in children of European descent aged 5-14 years was higher in Auckland than in South West Thames by a factor of 2.5 . The reported lifetime, 12 month and one month prevalences of wheeze were also higher in Auckland $(18.5 \%, 32.1 \%$ and $87.5 \%$ higher respectively). Unexpectedly, the hospital admission rate for asthma in children of European descent aged 5-14 years was $5 \%$ less in Auckland than in South West Thames. Comparative studies of hospital case notes and of general practitioners (using patient simulations) found that in Auckland the duration of illness before admission was greater and that general practitioners were less likely to admit. The overall standard of general practitioner care in Auckland was, if anything, higher than in South West Thames but in both areas there was considerable variation. On balance we conclude that the higher mortality rate in New Zealand is explained by higher levels of morbidity rather than relative deficiencies in care. Nevertheless, the implications of the lower use of hospital care for acute asthma observed in Auckland need further consideration.

Why is the mortality of tuberculosis not improving?

PDO DAvIES South Liverpool Chest Clinic, Liverpool Notification and mortality data for all forms of tuberculosis have been extracted from the relevant Office of Populations Censuses and Surveys Monitors (MB2, DH5) for the years $1974-86$ (the most recent year for which corrected data are available). The 
ratio of deaths due to tuberculosis (excluding late effects) to notifications for each year has been used as a measure of the mortality of tuberculosis. Over the 13 years studied there has been remarkably little change in this "mortality ratio," which has remained at approximately $7.5 \%$ of all notifications. The ratios for the $0-14$ and 15-34 age groups have improved from $1.5 \%$ to $0.7 \%$ for both groups. The ratio for the 35-54 age group has also shown improvement from $6 \%$ to $3 \%$ and for the 55-74 age group from $15 \%$ to $12 \%$. The ratio for the 75 year and over age group has remained constant at approximately $35 \%$. However, the proportion of notifications in the $75+$ group had doubled over the 13 years studied from $4.9 \%$ of all notifications to $10.0 \%$. As this group accounts for around $40 \%$ of all deaths from tuberculosis, despite the apparent improvement in mortality in younger age groups, the overall mortality for all age groups combined has shown virtually no improvement. From this and data published elsewhere (I Sutherland, VH Springette. J Epidemiol Commun Health $1989 ; 43: 15-24)$ it is estimated that the discontinuation of routine BCG within the next two years may result in one "preventable" death from tuberculosis, in the 15-34 age group, every two years, from about 1998 , for 10-15 years. The reason for the lack of improvement in tuberculosis mortality is the increase in the proportion of cases in the very old, where mortality rates are highest.

\section{Postal survey of asthma in the community}

RL LOVE, RM MURDOCH, SJ CAMPBELL, WG MIDDLETON, JS MILLAR, CA SOUTAR Institute of Occupational Medicine, Edinburgh; Bangour General Hospital, West Lothian; and Falkirk Royal Infirmary In the context of a proposed study of asthma and occupation we have tested the response to a postal self administered questionnaire designed to investigate respiratory symptoms compatible with a diagnosis of asthma. Thirteen hundred adults over the age of 16 , selected by stratified random sampling, from the electoral register of urban and rural communities in East Central Scotland, were invited to complete a two page questionnaire, based on that described by Burney and Chinn (Chest 1987;91 (suppl):795). Non-responders were sent reminders and a further questionnaire during the subsequent two months and the response rate at each stage was recorded. In total, 1026 returned completed questionnaires, corresponding to $79 \%$ of those invited. If those who had died or who had moved away were excluded from the total number invited, this would have given a response rate of $82 \%$. The prevalence of symptoms during the last year was examined within strata, and the overall figures are given here. Twenty two per cent reported wheezing and $13 \%$ reported having woken up with tightness in their chest. Ten per cent reported having an attack of shortness of breath and $5 \%$ reported being woken at night by such an attack. Prevalence of these symptoms was generally greater in men than in women, and men reported shortness of breath increasingly more frequently with age. Overall, $6 \%$ reported ever having had asthma. In conclusion, this postal survey has provided data on respiratory symptoms, for use in an epidemiological study, from more than three quarters of those individuals to whom questionnaires were sent.

(This work is supported by the Asthma Research Council.)

\section{Comparison of self admitted smoking} habits and exhaled carbon monoxide measurements in industrial employees in three European countries

WN TRETHOWAN, PS BURGE, I CALVERT, JM HARRINGTON Institute of Occupational Health, Birmingham University, and East Birmingham Hospital, Birmingham As part of an evaluation of smoking history in a respiratory morbidity survey amongst employees in the European ceramic fibres industry, measurements of exhaled carbon monoxide (CO) were collected from 621 participants working in seven manufacturing plants. Overall participation was $87 \%$ and included 268 in the UK, 286 in France, and 67 in West Germany. Participants were classified into never smokers, ex-smokers and current smokers, and current smokers by consumption of cigarettes per day. Comparisons were made between smoking groups in each country for mean exhaled $\mathrm{CO}$ levels and proportions of participants with individual measurements above 8 parts per million (ppm). From the questionnaire response, the proportion of participants in each country who were current smokers was $45 \%$ in the UK, $39 \%$ in France and $63 \%$ in Germany. The relationship between exhaled breath CO and numbers of cigarettes smoked was similar in the three national groups, suggesting that their patterns of smoking were similar. The proportion of ex-smokers with $\mathrm{CO}$ levels greater than $8 \mathrm{ppm}$, suggesting that they might be lying about their current smoking, was $8 \%$ in the UK, $6 \%$ in France, and $9 \%$ in Germany. The study shows that smoking is prevalent in ceramic fibre manufacturers, and that national differences exist in smoking habits in this industry.

Effect of alterations of dietary sodium on the severity of asthma

OJ CAREY, CR LOCK, JB COOKSON Glenfield General Hospital, Leicester Epidemiological and experimental evidence suggests that high levels of salt consumption are associated with increased bronchial reactivity and asthma mortality (Burney PGJ. Chest 1987;91:143S; Burney et al. Thorax 1989;44:36). To investigate the effect of dietary sodium on the severity of asthma, 27 asthmatic men (age range 12-67 y) placed on a low salt $(80 \mathrm{mmol})$ diet underwent a randomised double blind crossover trial to compare slow sodium supplements (SS) (200 mmol daily) with placebo $(\mathbf{P})$. Twenty two subjects completed the study. All parameters improved with $P$ compared with SS (for paired data, $F_{E V}$ : P 2.821 (SEM 0.27), SS 2.61 $1(0.26)$ (p < 0.05); PD $_{20}$ methacholine P $0.38 \mu \mathrm{mol}$ (0.75), SS $0.13 \mu \mathrm{mol}(0.55)$ (p $<0.05)$; puffs per day of bronchodilator: P 5.25 (range 211 ), SS $6.54(2.6-15)$ (p $<0.01)$; symptom scores on a six point scale: P 1.47 (0.01-3.4), SS $2.03(0.5-3.6)$ ( $p<0.01)$. PEF data were analysed as parallel groups for period 1 because of a treatment/period interaction of paired data. With $\mathbf{P}$ morning PEF rose by $2 \cdot 7 \%$ and evening by $5 \%$ above the pretrial value, and fell with SS by $3.4 \%(p<0.05)$ and $2.6 \%(p<0.01)$ respectively. The difference in urinary sodium excretion between treatments was $188 \mathrm{mmol}$. The study shows that changes in salt consumption alter the severity of asthma in men. A high salt intake results in physiological deterioration and increased morbidity.

Fenoterol and death from asthma in New Zealand, 1977-1981: a new casecontrol design

N PEARCE, J GRAINGER, M ATKINSON, J CRANE, C BURGESS, C CULLING, $H$ WINDOM, R BEASLEY Departments of Community Health and Medicine, Wellington School of Medicine, Wellington, New Zealand A previous New Zealand case-control study of asthma deaths in the 5-45 year age group during 1981-3 found that fenoterol by metered dose inhaler (MDI) was associated with an increased risk of death in severe asthmatics (Crane $\mathrm{J}$ et al. Lancet 1989;ii:917-22). A new case-control design has been used to evaluate the same hypothesis during 1977-81 using the same source for drug information for cases and controls. This involved identifying all relevant asthma deaths from national mortality records, and ascertaining those patients who had been admitted to a major hospital for asthma during the 12 month period prior to death. For each of these cases four age matched controls were then selected from persons admitted to hospital for asthma at the time of the case's death who had also had a previous admission for asthma in the past 12 months. For the 58 cases and 227 controls information on prescribed drug therapy was then collected from the hospital records relating to the previous admission. The relative risk of asthma death in patients prescribed inhaled fenoterol was 1.99 (95\% CI $1 \cdot 12-3.55, p=0.02)$. The inhaled fenoterol relative risk was 3.91 (95\% CI 1.79$8.54, \mathrm{p}<0.01$ ) in patients with a previous admission for asthma in the past 12 months, and $5.83(95 \%$ CI $1.62-21.0, p=0.01)$ in patients prescribed oral corticosteroids at the time of admission. In the group of patients with the most severe asthma (defined by a previous admission for asthma during the past 12 months and prescribed oral corticosteroids at time of admission) the inhaled fenoterol relative risk was $\mathbf{9 \cdot 8 2}(\mathbf{9 5} \% \mathrm{CI} 2 \cdot 23$ $43.4, p<0.01)$. These findings add further support to the hypothesis that inhaled fenoterol increases the risk of death in patients with severe asthma.

Potassium channel activation in human airway smooth muscle in vitro

PJ BARNES, CL ARMOUR, L ALOUAN, P JOHNSON, JL BLACK Department of Thoracic Medicine, National Heart and Lung Institute, London, and Department of Pharmacology, University of Sydney, Sydney, Australia Potassium $(\mathbf{K}+)$ channels are involved in recovery of excitable cells after depolarisation. Drugs which block these channels cause an increase in excitability, whereas activation of $\mathbf{K}+$ channels should reduce excitability. There is a great diversity of $\mathbf{K}+$ channels and this makes selectivity of drug action a realistic possibility. We have investigated the effect of a $\mathrm{K}+$ channel activating drug, BRL 38227 (L-enantiomer of cromakalim), on human bronchial smooth muscle in vitro. Subsegmental bronchi obtained at lung surgery were 
suspended in organ baths containing KrebsHenseleit solution at $37^{\circ} \mathrm{C}$ and changes in isometric tension were recorded. BRL 38227 caused a dose related relaxation of airways precontracted with histamine, the mean concentration causing half maximal relaxation (EC50) being $0.21 \mu \mathrm{M}(95 \%$ confidence intervals $0 \cdot 11-0 \cdot 38, n=8)$. BRL was equally effective against similar contraction induced by carbachol and neurokinin $\mathrm{A}$ with $\mathrm{EC}_{\text {s }}$ values of 0.55 and $0.41 \mu \mathrm{M}$ respectively, and gave relaxation responses which were 70 $90 \%$ of the maximal response to isoprenaline (1 mM). The relaxant effect of BRL 38227 was blocked by glibenclamide in a competitive manner, suggesting that an ATP sensitive $\mathbf{K}+$ channel was involved. The calcium channel blocker verapamil, at maximally effective dose $(10 \mu \mathrm{M})$, caused $40 \%$ of isoprenaline relaxation in comparison to $77 \%$ relaxation with BRL 38227 in the same tissues $(n=3)$. This suggests that $K+$ channel activation may not only reverse $\mathrm{Ca}++$ entry via voltage dependent $\mathrm{Ca}++$ channels but may also produce additional relaxation, which may relate to sequestration or extrusion of intracellular $\mathrm{Ca}++. \mathrm{K}+$ channel activators appear to be useful functional antagonists in human bronchi in vitro and may be effective bronchodilators in asthma therapy.

Phorbol myristate acetate (PMA) potentiates responses to cholinergic nerve stimulation in rabbit airway

KG CRABB, JC MCGRATH, NC THOMSON Department of Respiratory Medicine, Western Infirmary, Glasgow, and Autonomic Physiology Unit, University of Glasgow Receptor operated stimulation of different cell types causes hydrolysis of membrane phospholipids to produce inositol triphosphate and diacylglycerol. Diacylglycerol activates protein kinase $C$, which may be involved in the regulation of airway tone. To examine the role of protein kinase $\mathrm{C}$ in airway smooth muscle contraction we have examined the effects of the phorbol ester PMA, which activates protein kinase $C$, on cholinergic stimulation of isolated rabbit airway rings. Bronchial rings were suspended in baths containing oxygenated Krebs-Henseleit solution. Electrical field stimulation $(50 \mathrm{~V}, 16 \mathrm{~Hz}$, $0.1 \mathrm{~ms}$ for $10 \mathrm{~s}$ ) was applied and changes in tension were measured isometrically. PMA $\left(10^{-6}-10^{-4} \mathrm{M}\right)$ did not cause contraction of airway smooth muscle but it potentiated the contractile response to electrical field stimulation, reaching a plateau at approximately $60 \mathrm{~min}$. At this time point PMA $\left(10^{-4}\right.$, $10^{-5}, 10^{-6} \mathrm{M}$ ) produced a mean (SEM) maximum contraction from baseline values of $235(50) \%, 178(27) \%$ and $124(8) \%$ respectively. These responses were significantly ( $p$ $<0.05$ ) greater than the respective time control values: $93(5) \%, 74(10) \%$ and 77 (9) $\%$. Atropine $\left(10^{-7} \mathrm{M}\right)$ abolished the electrical field stimulation response and no potentiation was shown by PMA. These results indicate that the phorbol ester PMA potentiates responses to cholinergic nerve stimulation in rabbit airway smooth muscle.

Involvement of a $\mathbf{G}$ protein in pharmacomechanical coupling in bovine tracheal smooth muscle

IP HALL, $S$ HARDING, SJ HILL, AE TATTERSFIELD Department of Physiology and Pharmacology,
Queen's Medical Centre, and Respiratory Medicine Unit, City Hospital, Nottingham Agents such as histamine are thought to initiate a contractile response in airway smooth muscle by stimulating the hydrolysis of phosphatidylinositol 4,5-bisphosphate by phosphoinositidase $C$ to yield inositol $1,4,5-$ trisphosphate and diacylglycerol. The former is able to release calcium from intracellular stores, and the latter to activate protein kinase C. Activation of phosphoinositidase $C$ in many tissues is dependent on a $G$ protein termed $G_{p}$. In this study we have examined the involvement of $G_{p}$ in pharmacomechanical coupling in bovine tracheal smooth muscle (BTSM) by utilising the ability of fluoroaluminate $\left(\mathrm{AlF}_{4}^{-}\right)$to activate $G_{p}$. At concentrations reported to activate $G_{p}$ in other tissues $(>1 \mathrm{mM}), \mathrm{AlF}_{4}^{-}$produced concentration related contraction of strips of BTSM $(\mathbf{n}=6)$. The maximal contractile response seen with $\mathrm{AlF}_{4}{ }^{-}$accounted for 59 (SEM 7) \% of the response to $10 \mu \mathrm{M}$ carbachol. This contractile response to $\mathrm{AlF}_{4}{ }^{-}$was maintained in calcium free medium, and reversed by isoprenaline ( $50 \mathrm{nM}, \mathrm{n}=4$ ). In addition, $\mathrm{AlF}_{4}{ }^{-}$produced dose related formation of $\left[{ }^{3} \mathrm{H}\right]$-inositol phosphates at concentrations above $1 \mathrm{mM}$. The inositol phosphate response to $20 \mathrm{mM} \mathrm{AlF}_{4}$ accounted for 38 (7)\% of the maximal response to carbachol ( $1 \mathrm{mM}, \mathrm{n}=9$ ). As previously reported, the response to $20 \mathrm{mM}$ $\mathrm{AlF}_{4}^{-}$was inhibited by the beta 2 agonist salbutamol ( IC $_{50} 0.08 \mu \mathrm{M}$; Hall and Hill. $\mathrm{Br} J$ Pharmacol (in press)). Our data demonstrate that $\mathrm{AlF}_{4}^{-}$can induce both a contractile response and an inositol phosphate response in BTSM, and that these responses are both subject to regulation by beta-agonists. These results suggest that $G_{p}$ is involved in pharmacomechanical coupling in BTSM.

We are grateful for financial support from the Asthma Research Council.

Immunolocalisation of glutathione Stransferases in normal lung

D LAMB, DJ HARRISON University Department of Pathology, Edinburgh Glutathione Stransferases (GST) may be important in the regulation of lung injury caused by inflammatory processes involving leukotrienes (Int $J$ Biochem 1988;20:661) and by cigarette smoking (Carcinogenesis 1986;7:751). Individuals nulled for GST mu have an increased susceptibility to lung cancer. Previous studies have mainly relied on biochemical assessment of lung tissue. This fails to distinguish between the three functional compartments of lung: air conducting, gas exchange, and vascular. We have investigated the distribution of GST isoenzymes in lung by immunohistochemistry. Lung blocks were obtained from an uninvolved love of pneumonectomy specimens removed for carcinoma. All patients were smokers. Antibodies against pi class GST stained bronchial and bronchiolar epithelium strongly. Alveolar lining cells and alveolar macrophages in some cases also contained GST pi. Staining for GST alpha was restricted to bronchial and bronchiolar epithelium. GST mu was distributed similarly to GST pi but the intensity of staining was less and there was intercase and intracase variation. Some cases expressed no detectable GST mu. Microsomal GST was present in alveolar lining cells but was heterogeneous within single cases and between cases. Some cases had strong endothelial staining. Overall five cases were strongly stained for microsomal GST, two cases were weakly stained, and three cases had no detectable staining. These findings form the basis for further study of the role of GST in the pathogenesis of lung diseases such as emphysema.

Histamine release in isolated large airway segments of normal and asthmatic subjects in vivo

DL MAXWELL, BA ATKINSON, M BARROS, TH LEE Department of Allergy and Allied Respiratory Disorders, Guy's Hospital, London Asthmatic airways are hyperresponsive to a number of non-specific stimuli such as exercise and dry air hyperventilation. It is suggested that the common pathway is an increase in the osmolarity of fluid lining the bronchial epithelium. Challenge with these stimuli causes mediator release into the peripheral circulation, but little is known about mediator release within the airways in man. Adapting a technique of Smith et al (Eur Respir J 1988;1:792) we have obtained lavage fluid from isolated segments of large airways before, during and following challenge with hypertonic saline in six normal and five mild asthmatics with exercise induced asthma. Under bronchoscopic guidance a multichannel catheter tipped with a balloon was inserted into the left main bronchus. The balloon was then inflated just above the bifurcation of the bronchus. Six aliquots of normal saline (PRE) were instilled and aspirated from above the balloon (dwell time of 45-60 s). This was followed by six aliquots of $3 \mathrm{M}$ saline and then six more of $\mathrm{N}$ saline. Pooled samples from each of pre, hyper and post were assayed for histamine (radioimmunoassay) and geometric mean values (nM) given in the table.

\begin{tabular}{lccc}
\hline & Pre & Hyper & Post \\
\hline Normal & 1.2 & 2.0 & 1.5 \\
Asthma & 5.4 & 10.0 & 10.3 \\
\hline
\end{tabular}

Baseline and stimulated concentrations of histamine were higher in the asthmatics than those of normals ( $p<0.025$ ). One of six normal and four of five asthmatics showed significant increases in lavage histamine during and following hyperosmolar challenge. Two of five asthmatics developed mild bronchoconstriction following the procedure, which was otherwise well tolerated. These data show that the fluid from large airways of asthmatics contain greater concentrations of histamine than that of normals and that asthmatic epithelium responds to hyperosmolar stimuli with increased secretion of histamine.

Assessment of the bronchial mucosal bioelectric responses with a modified Ussing chamber

V TSANG, EWFW ALTON, ME HODSON, M YACOUB Brompton Hospital, London Measurements of bronchial mucosal bioelectric properties and responses to different mediators can provide useful information, particularly in patients with cystic fibrosis, 


\begin{tabular}{|c|c|c|c|c|}
\hline Tissues & & $\operatorname{SCC}\left(\mu A \mathrm{~cm}^{-2}\right)$ & $P D(m V)$ & $R$ (ohms $\mathrm{cm}^{2}$ ) \\
\hline Non-CF: & $\begin{array}{l}2 \mathrm{~mm} \text { sheet }(\mathrm{n}=15) \\
2 \mathrm{~mm} \text { biopsy }(\mathrm{n}=6) \\
4 \mathrm{~mm} \text { sheet }(\mathrm{n}=14) \\
2 \mathrm{~mm} \text { sheet }(\mathrm{n}=14) \\
2 \mathrm{~mm} \text { biopsy }(\mathrm{n}=10) \\
4 \mathrm{~mm} \text { sheet }(\mathrm{n}=8)\end{array}$ & $\begin{array}{l}17(15 \cdot 5) \\
17 \cdot 2(9 \cdot 5) \\
9 \cdot 4(5 \cdot 6) \\
22 \cdot 3(20 \cdot 9) \\
17 \cdot 2(10.5) \\
10 \cdot 3(3.8)\end{array}$ & $\begin{array}{l}0.36(0.26) \\
0.29(0.14) \\
0.56(0.45) \\
0.49(0.45) \\
0.38(0.14) \\
0.64(0.22)\end{array}$ & $\begin{array}{l}29.9(14.2) \\
19.8(8.3) \\
56.5(15.9) \\
25.2(10.6) \\
21.9(9.1) \\
66.2(23.5)\end{array}$ \\
\hline
\end{tabular}

(CF) before and after heart-lung transplantation (HLT). This may demonstrate whether the typical CF biochemical defect recurs in the transplanted lungs after HLT. A preliminary in vivo report suggested this may not be the case (Alton et al. Lancet 1987;i:1026). We have assessed the possibility of in vitro measurements of the basal properties (SCC, short circuit current; PD, potential difference; $R$, tissue resistance) and the pharmacological responses of the bronchial mucosal biopsy specimens obtained from the transplanted lungs. A pilot study involved $\mathrm{CF}$ and non-CF bronchial mucosal sheets with sizes ranging from 2 to $4 \mathrm{~mm}$ diameter, and bronchial mucosal biopsy specimens ( 2 $\mathrm{mm}$ diameter) using a rigid bronchoscopic biopsy forceps, obtained from the explanted lungs at the time of HLT. They were mounted in a modified Ussing chamber (tissue diameter of 2 or $4 \mathrm{~mm}$ ), and the bioelectric properties were assessed (Taylor et al, Gut 1988;29:957-962)-see table. In non-CF biopsy specimens, amiloride $(10 \mu \mathrm{M})$ caused a mean decrease in SCC of $62 \%$, and subsequent stimulation with isoprenaline (10 $\mu \mathrm{M}$ ) produced a mean increase of $60 \%$. In contrast, CF biopsy specimens produced a slightly greater fall in SCC of $66.5 \%$, but with no response from isoprenaline. The typical pharmacological responses of the $\mathrm{CF}$ and non-CF tissues were maintained (Knowles et-al. J Clin Invest 1983; 71:1410). Despite a lowered tissue resistance in the $2 \mathrm{~mm}$ diameter Ussing chamber, the other basal properties of both CF and non$\mathrm{CF}$ bronchial mucosal biopsy specimens did not differ from tissues in the $4 \mathrm{~mm}$ diameter chamber, which suggested the feasibility of this method.

\section{Occult carcinoma in patients with pul-} monary emboli

AG FENNERTY, HG SHETTY, G ROBERTS, IA CAMPBELL, PA ROUTLEDGE Llandough Hospital, Penarth, S Glamorgan Patients with a carcinoma are at an increased risk of developing thrombo embolic disease, but whether patients presenting with thrombo embolic disease should be investigated for underlying malignancy is controversial. To establish if there is an increased risk of carcinoma developing in patients presenting with pulmonary embolus (PE) 100 consecutive patients with PE confirmed by a high probability lung scan (mean age $56 \pm 14$ yrs, 47 female) were compared with 100 consecutive control subjects with low probability scans (mean age 51 (SD 16) y, 61 female) in a prospective study. At the time of presentation seven $P E$ and 11 control patients were known to have had a malignancy and were excluded from follow up. Ninety two PE patients were followed up for a mean of $47(18)$ months and 86 controls for a mean of 39 (16) months, with one and three patients lost to follow up in the two groups. Twenty one PE patients and 18 controls died of documented causes and three
PE patients and four controls developed carcinoma during follow up. The calculated incidence of newly diagnosed carcinoma in each group, taken from figures published in the OPCS cancer registry for 1984 was $2 \cdot 3$ and 2.01 respectively, giving a relative risk of 1.3 for PE patients and 2.0 for controls (NS). While this study cannot exclude the possibility that patients with $\mathrm{PE}$ have an increased risk of developing a malignancy, the incidence of carcinoma in these patients appears to be low $(0.86$ per 100 patient years $)$ and no higher than in patients with low probability scans. We conclude that investigation to detect occult carcinoma is not indicated in patients with high probability lung scans.

Low dose rate endobronchial radiotherapy using caesium-137

PJM GEORGE, BS MANTELL, RM RUDD London Chest Hospital and London Hospital, London Endobronchial radiotherapy has considerable potential as a palliative treatment in advanced tracheobronchial malignancy. Treatment with high activity iridium has been shown to be safe and effective in relieving upper airway obstruction; however, it requires costly equipment which is not widely available within the NHS. We have developed a technique using caesium-137, which employs standard afterloading equipment (Curietron) used to treat gynaecological malignancy. Treatments have been combined with endoscopic laser therapy with the intention of prolonging the palliative response. After completion of laser therapy under general anaesthesia, a flexible polythene catheter was passed into the trachea through a minitracheostomy incision. The catheter was guided into the affected airway with a flexible bronchoscope and, when correctly positioned, clamped to a plastic mount which was taped to the neck. After recovery from the anaesthetic, a caesium source of the control into the catheter. Treatment times were varied from 5-7.5 hours to deliver doses ranging from $2000-3000 \mathrm{cGy}$ at $0.5 \mathrm{~cm}$ from the source. This technique has been performed in seven patients with non-small cell lung cancer. Six derived immediate benefit, although one died after two weeks from a torrential haemoptysis. Two relapsed after seven and 10 weeks and required repeat lase treatments; one of these patients has subsequently been retreated with caesium using a higher radiation dose. The remaining three patients who responded have remained well for 14-30 weeks. The seventh patient, who Although treatment with caesium-137 is more invasive and time consuming than with being more widely available and much less expensive. We believe that the combination of endobronchial radiotherapy and laser therapy may provide durable palliation. appropriate length was loaded by remote did not respond, has not been retreated. high activity iridium, it has the advantages of
Does pulmonary inflammation influence benign lymph node size in patients with bronchogenic carcinoma?

KM KERR, CG WATHEN, WS WALKER, EW CAMERON, NJ DOUGLAS, D LAMB University of Edinburgh Lymph node size is often considered important when using imaging techniques to detect mediastinal lymph node metastases in patients with bronchogenic carcinoma who are being considered for surgery. We wished to examine whether inflammatory changes in the lungs of such patients produced benign lymphadenopathy. Forty four patients with operable bronchogenic carcinoma and no other cause for benign adenopathy (for example, sarcoidosis or anthracosis) had pulmonary resection and removal of all accessible mediastinal lymph nodes. Two pathologists scored, in a semiquantitative manner, the degree of inflammatory change in fixed lung slices and in representative histological sections. Each lymph node was measured and processed in toto. No correlation was found between benign lymph node size and chronic inflammatory changes in the lung, including endogenous lipoid pneumonia. However, maximum node size was greater in the 14 patients with significant acute inflammatory changes in the lung than in those without for both hilar $(p=0.02)$ and mediastinal $(p=0.01)$ lymph nodes. All of those patients with a positive score for acute pulmonary inflammation had at least one mediastinal lymph node of maximum diameter $15 \mathrm{~mm}$ or more. Acute inflammation distal to lung cancers is associated with significant reactive lymph node enlargement in both hilar and mediastinal nodes.

Initial staging of non-small cell lung cancer (NSCLC): value of routine radioisotope bone scanning

F MICHEL, M SOLER, E IMHOF, AP PERRUCHOUD Division of Respiratory Diseases, University Hospital, Basel, Switzerland The skeletal system is a preferred location for distant metastases in primary lung cancer. It is still a matter of controversy, however, whether in the initial staging of NSCLC radioisotope bone scans should be performed routinely or only when there is clinical suspicion of skeletal metastases (SM). The purpose of this study was to compare the sensitivity of clinical indicators of SM (bone pain in history or on physical examination, increased serum calcium, increased alkaline phosphatase) with routine bone scanning. We studied 110 consecutive patients referred for preoperative staging of NSCLC during 1983-5. Ninety five per cent of these patients were followed up for up to five years. Routine staging included history, physical examination, laboratory tests, CT of the thorax and upper abdomen and bone scanning. In patients with positive bone scans additional radiography, conventional and computed tomography, or biopsy was performed to confirm or exclude SM. On initial staging 37 of 110 bone scans $(34 \%)$ showed areas of increased uptake, only nine of which $(8 \%$ ) were confirmed to be metastases. At least one clinical indicator for SM was present in $54(49 \%)$ of the patients, including all patients with proved SM. Compared with bone scanning the sensitivity of these clinical indicators was $100 \%$ with a specificity of $54 \%$. Follow up data on 96 out of 101 patients $(95 \%)$ with no initial SM were available to validate our skeletal staging. 
Within one year three of 27 patients with nonconfirmed positive bone scans had SM. Two of these metastases were located in areas of initially increased uptake. All three patients had signs of SM and all had inoperable, advanced carcinoma. Four of 69 patients with initially negative bone scan developed SM within one year after staging (negative predictive value of routine bone scan $94 \%$ ). We conclude that in NSCLC bone scanning is necessary only in patients with clinica indicators of SM. This approach reduces the number of bone scans and consecutive examinations without loss of sensitivity.

\section{Bronchoscopic cryotherapy for} advanced lung cancer

DA WALSH, OM MAIWAND, AR NATH, P LOCKWOOD, M SAAB Harefield Hospital, Harefield, Middlesex Bronchial obstruction and haemorrhage from intraluminal tumours cause significant morbidity in advanced carcinoma of the lung. Radiotherapy is limited by maximum permitted doses and the insensitivity of some histological types. Endobronchial laser therapy may produce bronchial clearance but is associated with a significant morbidity and mortality. We report a prospective assessment of subjective and objective palliation with 81 bronchoscopic cryotherapy sessions in 37 consecutive patients. Twenty three patients $(70 \%)$ reported overall subjective improvement. Scores for dyspnoea and haemoptysis improved in $37 \%$ and $67 \%$ of patients respectively. Stridor was relieved in four of seven patients $(56 \%)$. Radiographic evidence of collapse resolved in two of 29 patients $(7 \%)$ and improved in a further five $(17 \%)$. Nineteen patients $(58 \%)$ showed improvement in at least one objective measure of lung function. Seven patients $(24 \%)$ showed an improvement of more than $15 \%$ and 0.2 litres in $\mathrm{FEV}_{1}$ and change in $\mathrm{FEV}_{1}$ correlated with change in dyspnoea score $(r=0.36, p<0.02)$. Twenty $(77 \%)$ of 26 patients reviewed by bronchoscopy showed some clearance of endobronchial obstruction. No significant adverse events were attributable to cryotherapy and there was no treatment related mortality. Bronchoscopic cryotherapy provides a safe and useful palliation of dyspnoea, stridor and haemoptysis in advanced endobronchial malignancy.

\section{Malignant mesothelioma in the south east in 1987: clinical and pathological experience of 245 cases}

DH YATES, K O'DWYER, FG WARD Medical Boarding Centre for Respiratory Diseases, Department of Social Security, London Until 1988 the lungs of all cases of suspected or proved mesothelioma in the South East Region were examined by the London Medical Boarding Centre for Respiratory Diseases (formerly Pneumoconiosis Panel). Details of the occupational history, clinical presentation and necropsy were obtained, and lung tissue was examined for type of mesothelioma and presence of asbestos bodies by an independent expert. In most cases good information was available, obtained from a variety of sources: clinical examination and occupational history taken in life, hospital records, and verification of occupational details by correspondence with former employers. A retrospective study was performed on 245 cases of mesothelioma for the year of death 1987. Male to female ratio was 14:1. Necropsies were performed in $98 \cdot 4 \%$. Occupational exposure to asbestos was documented in $78.5 \%$, with definite absence of exposure in $9 \%$ and neighbourhood exposure in $1.6 \%$. Pleural mesothelioma was far commoner than peritoneal or pericardial, being present in $95.5 \%$. The commonest mode of presentation was breathlessness and chest pain with accompanying pleural effusion; but pneumothorax, thrombocytosis and a chest wall mass were also observed. The previously reported predominance of right pleural mesothelioma was again observed (ratio right: left 1:4:1) Asbestosis was commoner in peritoneal mesothelioma, and overall was found in $7 \cdot 3 \%$. Asbestos bodies were present on light microscopy in $54.4 \%$, with plaques in $32.6 \%$. Average time from onset of symptoms to diagnosis was $51 / 2$ months, and diagnosis was confirmed most commonly by open biopsy. In $15 \%$ of cases the diagnosis was not made in life. Spread through the chest wall was documented at necropsy in $24 \%$. Distant metastases were present in $57 \%$, a proportion lower than reported in some other studies but higher than has been previously assumed. Industrial disablement benefit was claimed in less than half of the cases, despite written notice to widows that they were eligible to apply.

Comparison of dosimeter and tidal breathing methods for measuring non-specific bronchial responsiveness (NSBR)

JR BEACH, SC STENTON, CL YOUNG, EH WALTERS, DJ HENDRICK Chest Unit, Newcastle General Hospital, University of Newcastle upon Tyne There have been few attempts to standardise or compare results from the dosimeter and tidal breathing methods of measuring NSBR. We have consequently followed a conventional protocol with methacholine to make two pairs of measurements of NSBR in 20 subjects using (1) a locally designed dosimeter which generates $50 \mu \mathrm{l}$ of aerosol per dose, and (2) a Wright nebuliser from which aerosol is generated continuously and each dose comprises the amount inhaled from two minutes of tidal breathing (Clin Allergy $1977 ; 7: 235$ - - see table. NSBR is expressed as the dose or concentration respectively which provokes a $20^{\circ}{ }_{0}$, decrement in $\mathrm{FEV}_{1}\left(\mathrm{PD}_{20}\right.$, $\mathrm{PC}_{20}$ ). For our own method, $\mathrm{FEV}_{1}$ is taken as the mean of the three best measurements from six made 210-300 seconds after challenge onset. This is greatly influenced by the bronchodilation which occurs after the first $F_{1} V_{1}$ manoeuvre. For the tidal breathing method, $\mathrm{FEV}_{1}$ is taken as the lower of only two measurements - at 150 and 210 seconds. In order to measure $\mathrm{FEV}_{1}$ (and hence $\mathrm{PD}_{20}$, $\mathrm{PC}_{20}$ ) by both methods in this particular study, one measurement was made at 150 seconds and a further six from 210-300

\begin{tabular}{lllr}
\hline & & \multicolumn{3}{l}{ Geometric } \\
Method & $\begin{array}{l}\text { FEV }, \\
\text { measurement }\end{array}$ & $\begin{array}{l}\text { mean } \\
P D_{20}, P C_{20}\end{array}$ & $C R^{\star}$ \\
\hline Dosimeter & Best 3 of 6 & $34 \cdot 6 \mu \mathrm{g}$ & $3 \cdot 0$ \\
Dosimeter & Lower of 2 & $17 \cdot 3 \mu \mathrm{g}$ & $8 \cdot 8$ \\
Tidal & Best 3 of 6 & $0 \cdot 794 \mathrm{mg} / \mathrm{ml}$ & $4 \cdot 0$ \\
Tidal & Lower of 2 & $0.359 \mathrm{mg} / \mathrm{ml}$ & $11 \cdot 2$ \\
\hline
\end{tabular}

${ }^{\star}$ Exp coefficient of repeatability. seconds. The paired methacholine tests were carried out by different investigators, blind to any previous results. CR provides a measure of precision for each method and defines the $95^{\circ}$ o confidence interval for the second of a further pair of readings (first/CR-first $\times$ $\mathrm{CR}$ ). We conclude (1) that the greatest precision was achieved by the dosimeter coupled with the "best three of six" measurement method for $F E V_{1}$, the latter making the larger contribution; (2) that $\mathrm{FEV}_{1}$ measured as "best three of six" compared with the "lower of 2" approximately doubled the $\mathrm{PD}_{20}$ and $\mathrm{PC}_{20}$; and (3) that a $\mathrm{PC}_{20}$ of $1 \mathrm{mg} / \mathrm{ml}$ was equivalent to a $\mathrm{PD}_{20}$ of the order $40-50 \mu \mathrm{g}$.

\section{Assessment of bronchodilator response} by spirometric and impedance methods: a comparison

P MCLOUGHLIN, JS PRICHARD Department of Medicine, Trinity College, Dublin Spirometry is a common method of determining air flow obstruction and response to bronchodilators. Recently, another simple method-change in airway impedance (measured by forced oscillation)-has become available. It requires little cooperation and is an attractive alternative to spirometry. We have compared the two approaches. Spirometry was performed using a Vitalograph and impedance was assessed by the Siregnost (Siemens) system. In 40 normal subjects (ages 24-60,22 male, 18 female) two measurements each of $\mathrm{FEV}_{1}$ and respiratory impedance $(Z)$ were made separated by 20 min. In each case the difference between the first and the second was determined $\left(\triangle \mathrm{FEV}_{1}\right.$, $\triangle \mathrm{Z}$ ) and the mean and SD for the group calculated. Values were $\triangle \mathrm{FEV}_{1}=-5 \mathrm{ml}$ (125); $\Delta \mathrm{Z}=-0.008 \mathrm{kPa} / \mathrm{l} / \mathrm{s}(0.397)$. Thirty patients with obstructive airways disease (aged 26-75, $17 \mathrm{M}, 13 \mathrm{~F}$ ) were examined similarly and $\Delta \mathrm{FEV}_{1}=15 \mathrm{ml}(139) ; \Delta \mathrm{Z}=$ $0.023 \mathrm{kPa} / \mathrm{l} / \mathrm{s}(0.492)$. Each method was found to be equally reproducible in normal subjects and patients $(F=0.8, p>0.05 ; F=1.5, p>$ $0.05)$. The distribution of $\Delta \mathrm{FEV}_{1}$ and $\Delta \mathrm{Z}$ allowed a means of assessing response to bronchodilators. If the change in $\triangle \mathrm{FEV}$, or $\triangle \mathrm{Z}$ before and after a bronchodilator exceeded the $95 \%$ confidence level the patient was considered to show a significant response. Thus in 50 patients with obstructive airways disease, when assessment of reversibility by impedance was compared with spirometry, the former had a sensitivity of $50 \%$ a specificity of $72 \%$, a negative predictive value of $69 \%$ and a positive predictive value of $50 \%$. However, if the conventional spirometric method $(15 \%$ increase in $\mathrm{FEV}_{1}$ ) is applied assessment by impedance change shows sensitivity of $65 \%$, specificity of $83 \%$, positive predictive value $72 \%$, negative predictive value $78 \%$. This comparison in turn raises questions about the definition of significant bronchodilator response.

Effect of airway calibre on the sensitivity of the human cough reflex

NB CHOUDRY, RW FULLER, ST MARY'S BSC GROUP Departments of Medicine and Clinical Pharmacology, Royal Postgraduate Medical School, and St Mary's Hospital (Praed 


\begin{tabular}{|c|c|c|c|c|}
\hline & Before & \multicolumn{3}{|l|}{ After } \\
\hline & & $1 \min$ & 10 mir & \\
\hline $\begin{array}{l}\text { Saline } \\
\text { Methacholine } \\
\text { Salbutamol }\end{array}$ & $\begin{array}{ll}4.55 & (0.2) \\
4.58 & (0.3) \\
4.32 & (0.2)\end{array}$ & $\begin{array}{ll}4.53 & (0 \cdot 2) \\
4 \cdot 17 & (0 \cdot 2)^{\star} \\
4 \cdot 45 & (0 \cdot 2)\end{array}$ & $\begin{array}{l}4 \cdot 54 \\
4 \cdot 22 \\
4 \cdot 58\end{array}$ & $\begin{array}{l}(0 \cdot 2) \\
(0 \cdot 2)^{\star} \\
(0 \cdot 2)^{\star}\end{array}$ \\
\hline $\begin{array}{l}\text { Log } \mathrm{D}_{2} \text { (mean (SEM)) } \\
\text { Saline } \\
\text { Methacholine } \\
\text { Salbutamol }\end{array}$ & $\begin{array}{ll}0.25 & (0.12) \\
0.15 & (0.16) \\
0.30 & (0.18)\end{array}$ & $\begin{array}{ll}0.5 & (0.23) \\
0.4 & (0.26) \\
0.15 & (0.16)\end{array}$ & $\begin{array}{l}0.60 \\
0.21 \\
0.50\end{array}$ & $\begin{array}{l}(0.32) \\
(0.25) \\
(0.35)\end{array}$ \\
\hline
\end{tabular}

ses to exercise. The accentuation of $\mathrm{VE} / \mathfrak{V}^{5} \mathrm{CO}_{2}$ and $\dot{\mathrm{VE}} / \dot{\mathrm{VO}}_{2}$ and the relation to the relatively high VD/VT suggests inequality of ventilation and perfusion. These abnormalities may contribute to the sensation of breathlessness in CCF.

Familial aspects of peripheral chemosensitivity and/or central respiratory drive in the determination of arterial $\mathrm{Po}_{2}$ in chronic obstructive lung disease (COPD)

Street) London The sensitivity of the cough reflex is thought to be determined in part by airway tone. Studies have shown that artificially induced cough may be modified by agents which relax airways such as $\beta$ agonists and antimuscarinic agents. There is also evidence of coughing during bronchoconstriction during attacks of asthma. We have investigated the effects of altering airway calibre in normal volunteers by using the spasmogen methacholine and bronchodilator salbutamol. Baseline $\mathrm{FEV}_{1}$ and capsaicin cough challenge using single breaths of saline or 0.4-50 nmol capsaicin to determine the dose causing two or more coughs was measured in six normal subjects (five male, age 20-21 years). Saline, salbutamol $250 \mu \mathrm{g}$, and methacholine (dose which caused a $40 \%$ increase in airways resistance) were given in a randomised double blind manner. $\mathrm{FEV}_{1}$ and $\mathrm{D}_{2}$ were remeasured 1 and $10 \mathrm{~min}$ after treatment. The results are shown in the table. Significant increases or decreases in airway tone in normal volunteers does not cause a change in sensitivity of the cough reflex. This suggests that changes of airway tone within the normal range cannot explain differences in sensitivity of the cough reflex in patients with cough

We would like to acknowledge the help given by the intercalated BSc students from St Mary's Hospital: D Barron, D Gillen, M Harbord, L Seal, N Spittle, and A Stears.

Recovery from voluntary hyperventilation in normal and asthmatic subjects

SG CHURCH, WN GARDNER Department of Physiology, King's College, London Hyperventilation (HV) with a low end tidal $\mathrm{PCO}_{2}$ $\left(\mathrm{PeTCO}_{2}\right)$ is a recognised complication of mild asthma but the cause is uncertain. During the recovery from voluntary hyperventilation (VHV) in hyperoxia in normal man, there is a "window" during which chemical drive is low or absent and breathing is only under the influence of "feedforward" mechanisms. We studied the recovery from three minutes of VHV to a $\mathrm{PETCO}_{2}$ of $20 \mathrm{~mm} \mathrm{Hg}$ in four normal subjects and four young mild asthmatics in remission. In the latter, symptoms were absent and FEV , VC and PEF within normal ranges, but they were hyperresponsive to methacholine. Subjects breathed a warmed, humidified, hyperoxic gas mixture from an open circuit via a Fleisch pneumotachograph and mouthpiece. PETCO ${ }_{2}$ was measured by mass spectrometer. Respiratory drive and timing variables were averaged by computer over 30 second bins during 20-50 minutes of recovery. The pattern of recovery in the normal subjects was as previously reported (Gardner et al. Am Rev Respir Dis 1987; 135(suppl 4):A372) with an exponential rise of $\mathrm{PErCO}_{2}$ to a plateau and long expiratory times when $\mathrm{PCO}_{2}$ was below the central chemoreceptor threshold. In the asthmatic group, $\mathrm{FEV}_{1}$ and $\mathrm{PEF}$ remained unchanged during VHV recovery. The recovery of PET$\mathrm{CO}_{2}$ was faster over the first few minutes and then increased at a slower rate, prolonging the time to recovery back to normal $\mathrm{PETCO}_{2}$ During resting breathing before VHV, inspiratory time was slightly longer and expiratory time slightly shorter than in the control group. These differences were greatly exaggerated during the recovery from VHV and the long end expiratory pauses seen in normal subjects were absent. These changes were heightened in both groups when VHV was immediately preceded by inhalation of methacholine at a dose of $50 \%$ of $\mathrm{PC}_{20}$ for five minutes. In conclusion, these techniques can unmask abnormalities of respiratory patterning in very mild asthmatics with otherwise normal breathing and lung function. These abnormalities can occur in the absence of chemical drive.

Ventilatory responses to exercise in patients with chronic cardiac failure

JS ELBORN, M RILEY, CF STANFORD, DP NICHOLls Royal Victoria Hospital, Belfast Patients with chronic cardiac failure (CCF) are commonly limited by dyspnoea during exercise. However, whether ventilatory responses to exercise are abnormal in such patients is unclear. We have studied ventilatory responses to progressive and steady state exercise in 45 patients with CCF and 23 matched normal controls. Patients and controls had no evidence of pulmonary disease by resting pulmonary function tests. Ventilatory responses to progressive treadmill exercise were compared between normals and CCF patients. Subsequently comparisons were made during a 20 minute steady state exercise test at $50 \%$ of peak oxygen uptake $\left(\mathrm{PVO}_{2}\right)$. Minute ventilation (VE), carbon dioxide production $\left(\dot{\mathrm{V}} \mathrm{CO}_{2}\right), \dot{\mathrm{V}} \mathrm{O}_{2}$ and end tidal $\mathrm{Co}_{2}$ $\left(\mathrm{PETCO}_{2}\right)$ were measured on line throughout exercise. During progressive exercise, when compared at the same percentage of $\mathrm{PVO}_{2}$, patients with CCF had a significantly higher $\dot{\mathrm{V}} \mathrm{E} / \dot{\mathrm{VO}}_{2}$ and $\dot{\mathrm{V}} / \dot{\mathrm{V}}_{\mathrm{CO}_{2}}$, which was progressive with increasing severity of CCF. During steady state exercise absolute $\dot{V}_{E}$ was similar in the two groups: controls $408(80) \mathrm{ml} / \mathrm{min} /$ $\mathrm{kg}$ and patients $352(85) \mathrm{ml} / \mathrm{min} / \mathrm{kg}$. Differences in $\dot{\mathrm{VE}} / \mathrm{VO}_{2}, \dot{\mathrm{VE}} / \dot{\mathrm{VCO}}_{2}$ and dead space/ tidal volume ratio (VD/VT) are shown below, mean (SD).

\begin{tabular}{llll}
\hline & $\dot{V}_{E} / \dot{V}_{O_{2}}$ & $\dot{V}_{E} / \dot{V} C_{C O}$ & $V D / V T$ \\
\hline Controls & $25(3)$ & $30(4)$ & $0.23(1)$ \\
CCF & $35(7)^{\star}$ & $46(9)^{\star}$ & $0.35(0.05)^{\star}$ \\
\hline
\end{tabular}

$\mathrm{p}<0.01 v$ controls

$\dot{\mathrm{VE}} / \dot{\mathrm{VCO}}_{2}$ strongly correlated with $\mathrm{VD} / \mathrm{VT}(\mathbf{r}=$ $0.87, \mathrm{p} 0.0001)$. We conclude that patients with CCF have abnormal ventilatory respon-
JE HILL, K SKWARSKI, TP KIRBY, W MACNEE, PM Respiratory Medicine, City Hospital, Edinburgh The inherited intensity of the carotid body mediated hypoxic ventilatory response may partly determine the $\mathrm{PaO}_{2}$ of patients with COPD. We have related the $\mathrm{PaO}_{2}$ (stable, on air) of 24 patients with COPD (FEV 12 $43^{\circ}$ o pred; $\mathrm{PaO}_{2} 5 \cdot 1-9 \cdot 5 \mathrm{kPa} ; \mathrm{PaCO}_{2} 4.9-8.7$ $\mathrm{kPa}$ ) to the ventilatory responses to both transient (three breaths N2) and rapid onset, three minute isocapnic step change $\left(\mathrm{FIO}_{2}\right.$ $20 \cdot 9-15 \%$ and $20 \cdot 9-12 \%$ ) hypoxia measured during moderate exercise $\dot{\mathrm{VO}}_{2} 11.9$ (SEM 1.8 ) $\left.\mathrm{ml} \mathrm{min} \mathrm{mg}^{-1}\right)$ in their offspring (20M, 15F, age 18-47 y; FEV $77-112 \%$ pred). Measurements were also made in 24 controls who were age and sex matched with the offspring, but whose parents did not have COPD. The patients' $\mathrm{PaO}_{2}$ correlated with $\dot{\mathrm{V}} / \mathrm{SaO}_{2}$ relationship in response to step change $(r=-0.48, p<0.01)$ but not transient hypoxia in the offspring. The dynamic ventilatory response to the two hypoxic stimuli was analysed by means of a mathematical model consisting of two differential equations in parallel ( 1 and 2 ) with gains $G 1$ and G2. Equation 1 describes the rapid onset response (time constant $<3 \mathrm{~s}$, possibly reflecting peripheral drive) and equation 2 the sustained response (possibly reflecting peripheral and central mechanisms). The patients' $\mathrm{PaO}_{2}$ was best described ( $\mathrm{p}<0.02$ ) by the multiple regression equation:

$\mathrm{PaO}_{2}$ (patient) $\left.=(0.35)(0 \cdot 14)\right) \mathrm{G} 2+(0.30)$

$$
(0 \cdot 15)) \mathrm{Gl}+(6.08)(0.35))
$$

There was no significant difference between the offspring $(\mathrm{O})$ and the age and sex matched controls (C) for the $\dot{\mathrm{VE}} / \mathrm{SaO}_{2}$ relationship to step change hypoxia $(\mathrm{O}, 0.37$ to -1.75 ; $\mathrm{C}$, -0.08 to $\left.-1.59,1 \min ^{-1} \%{ }^{-1}\right), \mathrm{Gl}(\mathrm{O}$, -1.45 to $4.17 ; \mathrm{C}, 0.24$ to $3.94,1 \mathrm{~min}^{-1} \%^{-1}$ ), or $\mathrm{G} 2(\mathrm{O},-1.83$ to 3.85 ; C. -2.27 to 3.09 $\left.1 \mathrm{~min}^{-1} \%{ }^{-1}\right)$. The $\mathrm{PaO}_{2}$ in COPD seems to depend on a genetically determined variation in the normal population of both peripheral hypoxic sensitivity and central respiratory control mechanisms.

Is the carbon dioxide response relevant to ventilation and sensation during exercise in normal man?

JE CLAGUE, MG PEARSON, PMA CALVERLEY Regional Thoracic Unit, Fazakerley Hospital, Liverpool Previous studies have shown that the ventilatory response to hypercapnia $(\dot{V} E /$ $\mathrm{PCO}_{2}$ ) correlates with the rate of increase of ventilation with $\mathrm{Co}_{2}$ production during exercise $\left(\dot{V}_{\mathrm{E}} \dot{V}_{\mathrm{CO}}\right)$ (Rebuck et al. Clin Sci 1972) but neither relates to perceived breathlessness in COPD patients (Robinson et al. Am Rev Respir Dis 1987). We have investigated these relationships in 11 normal subjects (10 M). Each performed duplicate $\mathrm{Co}_{2}$ rebreathes and cycle exercise tests both free breathing WARREN Rayne Laboratory, Department of 
(FB) and with a $10 \mathrm{~cm} \mathrm{H} \mathrm{H}_{2} \mathrm{O} / 1 / \mathrm{s}$ inspiratory resistive load (IRL). We recorded VE, $\mathrm{PETCO}_{2}$, mouth occlusion pressure (Po.1) and inspiratory effort sensation (IES) using a Borg scale. During rebreathing IRL depressed the ventilatory response $\dot{V} \mathbf{E} / \mathbf{P C O}_{2}$ by $25 \%$ while the IES/PCO slope increased by $57 \%$. During exercise IRL depressed $\mathrm{VE} / \mathrm{VCO}_{2}$ by only $9 \%$ yet increased IES $/ \mathrm{VCO}_{2}$ by $95 \%$. Neither the FB nor the IRL ventilatory (VE/ $\mathrm{PCO}_{2}$ ) or effort sensation responses (IES/ $\mathrm{PCO}_{2}$ ) during rebreathing were related to $\dot{\mathrm{V}} \mathrm{E} /$ $\mathrm{VCO}_{2}$ during exercise. Thus the hypercapnic ventilatory response does not predict the ventilatory response to isocapnic exercise. During both rebreathing and exercise IRL had no influence on the slope of the IES/Po.1 response. Differences in individual breathing pattern responses to IRL during exercise and hypercapnic rebreathing may explain the lack of association between the ventilatory responses and perceived effort (IES). Inspiratory effort sensation during both exercise and hypercapnia remained related to respiratory centre output as reflected by Po.1

Relative effects of inspiratory sensation and respiratory drive on peak exercise ventilation

JE ClAGUE, MG PEARSON, PMA CALVERLEY Regional Thoracic Unit, Fazakerley Hospital, Liverpool Exercise performance is often assessed as the maximum ventilation (VEmax achieved. Interpretation of VEmax especially in patients with chronic obstructive lung disease (COPD) is difficult. The respiratory performance of normal subjects is limited by the onset of inspiratory muscle fatigue (Bai et al. JAP 1983) but patients may be more limited by breathlessness. We have investigated 11 normal subjects $(10 \mathrm{M})$ who performed duplicate $\mathrm{CO}_{2}$ rebreathes and progressive cycle exercise tests both free breathing (FB) and with a $10 \mathrm{~cm} \mathrm{H}_{2} \mathrm{O} / 1 / \mathrm{s}$ inspiratory resistive load (IRL). We recorded VE, PET$\mathrm{CO}_{2}$, mouth occlusion pressure (Po.1) and inspiratory effort sensation (IES) using a Borg scale. VEmax, Po.1max and peak heart rate (HR) were reproducible between duplicate exercise tests with a coefficient of variation $(\mathrm{CV})$ of $7 \%, 17 \%$ and $2.5 \%$ respectively. During exercise IRL significantly depressed VEmax (53.2 FB to $31.71 / \mathrm{min}$ IRL) and $\dot{\mathrm{V} C O_{2}} \max$ (1882 FB to $1243 \mathrm{l} / \mathrm{min}$ IRL) while IESmax (4.6 FB to $6.5 \mathrm{IRL}$ ) ar.d Po. 1 max $\left(10.2 \mathrm{FB}\right.$ to $17 \cdot 1 \mathrm{~cm} \mathrm{H}_{2} \mathrm{O}$ IRL) increased (all $p<0.01)$ ). Peak values were not predicted by the $\mathrm{VE} / \mathrm{PCO}_{2}, \mathrm{IES} / \mathrm{PCO}_{2}$ or $\mathrm{Po} .1 / \mathrm{PCO}_{2}$ responses during hypercapnia. However, the "total inspiratory effort per minute" (IESmax $\times$ VEmax) was the same during FB and IRL exercise (242 FB $v 251$ IRL). Similarly the "total inspiratory drive per minute" (Po.1max $\times \dot{V}_{\text {Emax }}$ ) was unaltered by IRL (546 FB $v 590$ IRL). These "product values" varied between individuals (161 to 394 for IESmax $\times$ VEmax) but were reproducible for the four exercise tests (CV $27 \%$ ). The peak respiratory performance is dependent on changes in both IES and $\dot{V}_{E}$ and both need to be considered when assessing the respiratory disability of patients.

Metabolic and ventilatory adaptations to physical training in asthma

CJ CLARK, LM COCHRANE Department of Respiratory Medicine, Hairmyres Hospital, Glasgow In normal subjects physical training produces metabolic adaptations that may reduce the ventilatory demands of exercise. In asthma, however, there may be impairment of a variety of metabolic responses (Haas et al. Am Rev Respir Dis 1988), and beta ${ }_{2}$ selective agonists also produce changes in lactate metabolism (Holgate et al. Clin Sci 1981). It is therefore not clear what impact the disease has on the metabolic and ventilatory adaptations to physical training. This study reports the effects of physical training on 36 asthmatic subjects ( 14 male, 22 female) randomised into training and control groups. Metabolic and ventilatory responses during progressive incremental exercise were measured at 0 and three months. At work rates corresponding to $20 \%$ and $40 \%$ of initial $\mathrm{VO}_{2}$ max no significant change in blood lactate, carbon dioxide output $\left(\mathrm{VCO}_{2}\right)$ or minute ventilation ( $\dot{V E}$ ) occurred. At $60 \%$ of $\mathrm{VO}_{2}$ max there was a significant fall in $\dot{V C O}_{2}$ (p $<0.01)$ and $V_{E}(p<0.01)$. At $80 \%$ and $95 \%$ of initial $\mathrm{Vo}_{2} \max$ there was a significant fall (p $<0.001)$ of all three indices. No significant changes were seen at any of these work rates in the control group after training. This study shows that physical training can produce metabolic adaptations in asthmatic patients similar to those reported in normal subjects. This results in a reduction in the ventilatory response to exercise at high work loads, the magnitude of which may be advantageous for endurance exercise in the asthmatic patient.

\section{Effects of mode of exercise on breathing patterns during progressive incremen- tal exercise testing}

LM COCHRANE, CJ CLARK Department of Respiratory Medicine, Hairmyres Hospital, Glasgow A recent study (Cochrane et al. BTS Proceedings, Thorax 1989;44:885P) showed more breathlessness during progressive incremental exercise with bicycle ergometry than with treadmill walking. This study investigates breathing patterns produced by the two modes of exercise. Forty five healthy subjects ( 25 male, 20 female) performed the two progressive incremental exercise tests in random order within seven days. From a quadratic analysis of the relation of tidal volume, $\mathrm{TV}$ to minute ventilation three characteristics were compared: (1) plateau height (peak tidal volume); (2) turning point (minute ventilation at peak tidal volume); and (3) slope (of ascending curve) Results are given in the table. At equivalent levels of ventilation different patterns of breathing were demonstrated during bicycle and treadmill exercise. Peak tidal volume was reached at lower minute ventilation and the slope of tidal volume related to minute ventilation was greater on the bicycle than on the treadmill. There was no difference in peak tidal volume. The earlier, steeper increase in tidal volume during bicycle exercise may in part explain our previous observation that there is a lower threshold for the onset of breathlessness on bicycle exercise followed thereafter by proportionately greater breathlessness at equivalent ventilation throughout progressive incremental exercise.

\begin{tabular}{|c|c|c|c|c|}
\hline & & Peak TV & $\dot{V}_{E}$ at peak $T V$ & Slope \\
\hline $\begin{array}{l}\text { MALES } \\
\quad(\text { Mean }(\text { SEM }))\end{array}$ & $\begin{array}{l}\text { Bicycle } \\
\text { Treadmill }\end{array}$ & $\begin{array}{ll}2.84 & (0.55) \\
2.78 & (0.49) \\
\text { (NS) } & \end{array}$ & $\begin{array}{rr}99.5 & (34) \\
116.8 & (30) \\
(\mathrm{p}<0.004)\end{array}$ & $\begin{array}{ll}0.003 & (0.000) \\
0.002 & (0.000) \\
(p<0.018)\end{array}$ \\
\hline
\end{tabular}

\begin{tabular}{|c|c|c|c|c|}
\hline (Mean $($ SEM $))$ & $\begin{array}{l}\text { Bicycle } \\
\text { Treadmill }\end{array}$ & $\begin{array}{ll}1.89 & (0.37) \\
1.88 & (0.32) \\
\text { (NS) } & \end{array}$ & $\begin{array}{rr}71.7 & (16) \\
89.9 & (22) \\
(\mathrm{p}<0.001)\end{array}$ & $\begin{array}{l}0.0004 \quad(0.000) \\
0.0002 \quad(0.000) \\
(p<0.002)\end{array}$ \\
\hline
\end{tabular}

R TIPSON, D DUGMORE, A HARDMAN, MF BONE

\section{Effect of posture on regional ventilation} mal, unilateral or bilateral lung disease)
Use of a low intensity submaximal cycle ergometer test to monitor progress of patients with poor exercise tolerance through an exercise training programme Department of Thoracic Medicine, Russells Hall Hospital, Dudley, West Midlands, and Department of PE and Sports Science, Loughborough University, Loughborough Exercise training may improve exercise tolerance in patients with a wide range of chronic disease, totably ischaemic heart disease and obstructive airways disease (COAD). Optimal training depends on an assessment of functional fitness capacity and achievement of $60-70 \%$ work rates. Previously estimation of maximal oxygen uptake from extrapolation of submaximal heart rate data has been used but responses in a patient population are fickle and often inaccurate and a true $\mathrm{Vo}_{2} \max$ is almost impossible in this type of patient with such low exercise capacity. We decided to evaluate the sensitivity of a submaximal steady state cycle ergometer test with low exercise work loads of approximately 25, 50 75,100 watts in monitoring such patients with low exercise tolerance in a mixed training programme of 12 weeks' duration using individualised work intensities of $60-70 \%$ for 30 minutes three times weekly. During the exercise stress test heart rate (HR), minute ventilation $(\dot{\mathrm{V}} \mathrm{E})$, oxygen uptake $\left(\dot{\mathrm{Vo}}_{2}\right)$ and blood lactate levels were measured. After 12 weeks significant reductions in mean (SEM) HR (94 (4) $v 110$ (6) b. $\mathrm{min}^{-1}, \mathrm{p}<0.01$ ); $\mathrm{VE}_{\mathrm{E}}$ $29.8(1.8)$ v $\left.37.8(2.6) 1 . \mathrm{min}^{-1}, \mathrm{p}<0.01\right)$; and blood lactate concentration $(1.34(0 \cdot 17) v 2.50$ $(0 \cdot 16) \mathrm{mmol}^{-1}, \mathrm{p}<0.01$ were seen at stage 4 These results indicate that training effects can be detected with low intensity mixed exercise at a level which may be appropriate to chronic obstructive airways disease patients. In patient groups such a low incremental submaximal stress test is sufficient to enable blood lactate concentration to be employed as an index of fitness. in children and adults

H DAVIES, P HELMS, I GORDON Hospital for Sick Children, London Davies et al (Davies et al. Regional ventilation in infancy: reversal of adult pattern. $N$ Engl J Med 1985;313: 1626) have demonstrated that ventilation is preferentially distributed in infants to uppermost lung regions, the opposite pattern to that seen in adults. Groups of older children, adolescents and adults were therefore studied to determine at what age this pattern changes. Supine, right and left lateral krypton $81 \mathrm{~m}$ ventilation lung scans were performed on 43 children (mean age 10.5 years) attending the Hospital for Sick Children, and $\overline{1} \overline{6}$ adult volunteers (mean age $30 \cdot 7$ ). The children were divided into three subgroups on the interpretation of their chest radiograph (nor- 
Pulmonary function tests were performed in those children able to cooperate. In children aged 2-10 years distribution of the radionuclide to the right lung (VfR) supine was (mean (SEM)) $46 \cdot 1 \%(12 \cdot 6)$. When dependent VfR fell to $36 \cdot 0^{\circ}{ }_{0}(12 \cdot 8 \%)$, rising when uppermost to $56 \cdot 1 \%(9.9 \%)$. Both changes were significant $(\mathrm{p}<0.0005)$. In children aged $10-18$ years $\mathrm{VfR}$ (supine) was $57 \cdot 2 \%$ $\left(7 \cdot 3^{\circ}{ }_{0}\right)$, falling to $48 \%(8.2 \%)$ when dependent and rising to $62.9 \%(7.9 \%)$ when uppermost. These changes were also significant $(\mathrm{p}<0.0005)$. A different pattern was observed in adults. VfR (supine) was $52.4 \%$ $\left(1.5^{\circ}{ }_{0}\right)$, rising when dependent to $53.4 \%$ $\left(4.9^{\circ} \circ\right)$ and falling when uppermost to $48.9 \%$ $\left(7 \cdot 7^{\circ}\right)$. The change in ventilation from supine to uppermost and from dependent to uppermost reached significance $(p<0.05)$, although the change from supine to dependent did not. Neither chest radiograph appearances nor pulmonary function test results altered this pattern. The physiology of these differences and clinical consequences will be discussed.

Increases in nasal and pulmonary resistance in the supine posture in asthmatic subjects

CJ DUgGan, A Watson, SB PHAGOO, NB PRIDE Department of Medicine, RPMS, Hammersmith Hospital, London Subjects with asthma frequently have nasal symptoms and complain of orthopnoea, even when they are awake. However almost all assessments of airways resistance in asthmatic subjects are made in the sitting posture. We have examined changes in total respiratory resistance (Rrs) and midtidal lung volume (MTLV) when breathing via the nose or via the mouth in the sitting and supine posture in 10 subjects with both asthma and nasal symptoms ( 6 males, mean age 62.8 years: range 38 80 years) mean $\mathrm{FEV}_{1}{ }^{\circ}$ o predicted $54.6 \%$ and mean $\mathrm{FEV}_{1} / \mathrm{VC} 64^{\circ}{ }_{0}$. Rrs was measured at $6 \mathrm{~Hz}$ using the pseudorandom noise oscillation technique of Landser ( $J$ Appl Physiol 1976;43:101) and values were obtained breathing via the nose or mouthpiece in the erect and supine posture. In both postures each subject had a higher Rrs via the nose compared to the mouth, and Rrs was higher in the supine posture for each breathing route. In the erect posture resistance breathing via the nose was more than twice the value breathing via a mouthpiece. In the supine posture nasal breathing was again more than twice the resistance of oral breathing in that posture. Normal subjects also have rises in both nasal and pulmonary resistance and a fall in MTLV in the supine posture. However, the increase in resistance both in absolute and percentage terms was larger in our asthmatic subjects. Thus, in these patients with chronic stable asthma, total resistance during nasa breathing in the supine posture reached high levels when awake; during sleep these values are likely to be even higher.

Supported by the Asthma Research Council and a Royal North Shore Hospital Centenary Fellowship.
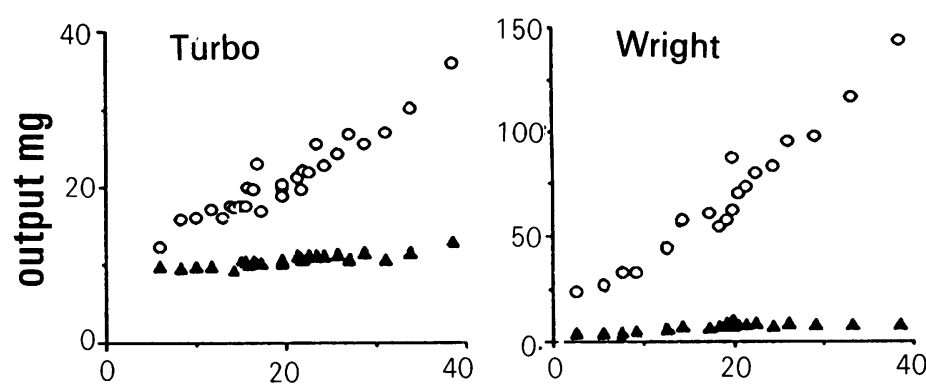

O weight

$\Delta$ aerosol

Effect of temperature on jet nebuliser output

JH DENNIS, SC STENTON, EH WALTERS, DJ HENDRICK Chest Unit, Newcastle General Hospital, and Division of Environmental and Occupational Medicine, University of Newcastle upon Tyne A number of recent reports describe increasing jet nebuliser output with increasing temperature of the reservoir solution, output being measured by the total weight lost during nebulisation. Bradley and Durham further suggest that temperature exerts an important confounding effect when non specific bronchial responsiveness (NSBR) is measured using a Wright nebuliser and the tidal breathing method (BTS proceedings, Thorax $1989 ; 44: 864 \mathrm{P})$. This has stimulated us to use a new technique (impaction of aerosol onto glass fibre filters coupled with assay of a fluoride chemical tracer) for assessing the aerosol component of the weight lost (aerosol + vapour) from both a Wright nebuliser and a Turbo nebuliser. The latter is used in our dosimeter method of measuring NSBR. Output was measured over intervals of 2 seconds at an air flow rate of $7 \mathrm{lpm}$ for the Turbo and of 20 seconds at $9 \mathrm{lpm}$ for the Wright. A thermocouple within the nebuliser reservoir monitored temperature changes over the range $2-40^{\circ} \mathrm{C}$. Weight loss increased markedly with temperature for both Turbo ( 3 fold) and Wright ( 6 fold) nebulisers, but the aerosol component increased only trivially (Turbo $9 \cdot 6-12.7 \mathrm{mg} / 2 \mathrm{~s}$, Wright $4 \cdot 1-8.2 \mathrm{mg}$ / $20 \mathrm{~s})$. Over normal operating temperatures $\left(10-20^{\circ} \mathrm{C}\right)$ this effect on aerosol output is inconsequential. Since dosimeters nebulise only tiny proportions of the nebuliser solutions, vapour loss will not produce any appreciable change in concentration and measurement of NSBR should not be affected by temperature change. With tidal breathing however, a considerable concentrating effect may occur and this would be exaggerated with increases in operating temperaturethereby influencing the measurement of NSBR.

\section{Temperature profile of a maximal} forced expiratory manoeuvre

I MADAN, J LLOYD, AC PINCOCK, MR MILLER University of Birmingham, Department of Medicine, Good Hope Hospital, Sutton Coldfield, West Midlands The temperature profile during a maximal forced expiratory manoeuvre is not known and it may influence the performance of flow measuring devices and the application of any temperature correction. We have used a fine thermocouple (5 $\mu \mathrm{m}$ diameter) placed $10 \mathrm{~mm}$ from the mouth end of a $28 \mathrm{~mm}$ i.d. mouthpiece to record air temperature on expiration. The time constant of the thermocouple was $7 \mathrm{~ms}$ on immersion in cold water, $260 \mathrm{~ms}$ in an air flow of $0.5 \mathrm{l} / \mathrm{s}$ and $60 \mathrm{~ms}$ in a flow of $10.2 \mathrm{l} / \mathrm{s}$. In 12 normal subjects, who performed a rapid inhalation through the mouth followed by immediate exhalation, the temperature at PEF was $33.4(0.9){ }^{\circ} \mathrm{C}$ (mean (SD)) and was also $33.4(1.1){ }^{\circ} \mathrm{C}$ at FVC. At $25 \%, 50 \%$ and $75 \%$ of FVC the temperature was significantly higher ( $p<0.05$, Mann-Whitney), with a peak of $34.4(0.4)^{\circ} \mathrm{C}$ at $75 \%$ of FVC. In 10 patients with chronic airflow limitation (mean $\mathrm{FEV}_{1} 1.0(0.5)$ 1) the temperature profile was not significantly different from that of the normal subjects, being $33.0(1.7)$ ${ }^{\circ} \mathrm{C}$ at PEF and $34.5(0.8){ }^{\circ} \mathrm{C}$ at $75 \%$ of FVC. When the normal subjects inhaled ambient air slowly through the nose all the temperatures, except that at FVC, were significantly higher than before, with the maximum increase of $0.8^{\circ} \mathrm{C}$ being at PEF. Rapid inhalation of air at $6^{\circ} \mathrm{C}$ through the mouth followed by immediate exhalation did not significantly lower the temperature profile as compared to that found when ambient air was inhaled in this way. We conclude that from PEF to FVC the expired air temperature varies by up to $1.0^{\circ} \mathrm{C}$ and it is sensitive to the mode of inhalation but not to a $20^{\circ} \mathrm{C}$ drop in the inspired air temperature.

Distribution of changes in tracheal blood flow in dogs during isocapnic hyperventilation and PEEP

DJ GODDEN, EM BAILE, PD PARE UBC Pulmonary Research Laboratory, St Paul's Hospital, Vancouver We examined the effects on tracheal blood flow of $(a)$ isocapnic hyperventilation of warm dry air and (b) $15 \mathrm{~cm} \mathrm{H}_{2} \mathrm{O}$ positive end expiratory pressure (PEEP) in dogs. Five mixed breed dogs were anaesthetised, paralysed, and ventilated through a tracheostomy. Tracheal blood flow was measured by the radiolabelled microsphere reference flow technique (Baile et al. JAP 1982;53:1044). Measurements of aortic blood pressure, cardiac output and tracheal blood flow were obtained after 20 minutes baseline ventilation (rate $15 / \mathrm{min}, \mathrm{VT} 15 \mathrm{ml} / \mathrm{kg}$ ), 20 minutes' isocapnic hyperventilation (rate $40 /$ $\mathrm{min}, \mathrm{V}_{\mathrm{T}} 25 \mathrm{ml} / \mathrm{kg}$ ) and 20 minutes' ventilation during which $15 \mathrm{~cm} \mathrm{H}_{2} \mathrm{O}$ of PEEP was applied (rate $15 / \mathrm{min}, \mathrm{V}_{\mathrm{T}} 20 \mathrm{ml} / \mathrm{kg}$ ). The dogs were then killed by an overdose of anaesthetic, and the trachea was excised and divided into six sections (right and left upper, middle and lower). Tissues were processed to allow blood flow to the mucosa, the cartilaginous portion, and the adventitia in each section to be separately determined. Under baseline conditions, the ratio of blood flow expressed per unit mass of tissue, in mucosa: cartilage: adventitia was 15:0 6:1. Hyperventilation of warm dry air increased blood flow to the mucosa (mean (SEM) increase from baseline $165(32) \%$ ) but caused no significant overall change in blood flow to cartilage and adven-

\begin{tabular}{|c|c|c|c|c|}
\hline & \multicolumn{2}{|c|}{$\operatorname{Rrs}(6 \mathrm{~Hz})\left(\mathrm{cm} \mathrm{H}_{2} \mathrm{O} / \mathrm{l} / \mathrm{s}\right.$, mean $\left.(S E M)\right)$} & \multicolumn{2}{|c|}{$M T L V(l$, mean $(S E M))$} \\
\hline & Mouth & Nose & Mouth & Nose \\
\hline $\begin{array}{l}\text { Sitting } \\
\text { Supine }\end{array}$ & $\begin{array}{l}3.69(0.22) \\
5.36(0.26)\end{array}$ & $\begin{array}{r}7.56(1.40) \\
11.17(1.85)\end{array}$ & $\begin{array}{l}3.71(0 \cdot 19) \\
3.49(0.20)\end{array}$ & $\begin{array}{l}3.72(0.22) \\
3.51(0.22)\end{array}$ \\
\hline
\end{tabular}


titia. PEEP markedly reduced blood flow to all levels of the airway wall, the reduction from baseline being 65 (5) \% in the mucosa, $58(5) \%$ in the cartilage, and $36(9) \%$ in the adventitia. The results suggest that, during hyperventilation, vasomotion occurs in the mucosal vessels independent of the vessels in the underlying wall. During PEEP substantial falls in blood flow occur through the full thickness of the tracheal wall. The functional significance of this degree of ischaemia requires further investigation.

DG was supported by the Wellcome Trust.

\section{Endobronchial pH}

P MCLOUGHLIN, P BYRNE, A STUART, J PRISCHARD Departments of Medicine and Surgery, Trinity College, Dublin We have measured endotracheal and endobronchial surface $\mathrm{pH}$ in patients without pulmonary disease, with chronic obstructive airways disease and with pneumonia. We used a monocrystant, unipolar antimony electrode (Synectics Ltd, Sweden, $2 \cdot 1 \mathrm{~mm}$ tip diameter). This was inserted through the channel of an Olympus BF3 fibrescope and a $\mathrm{Ag} / \mathrm{AgCl}$ reference electrode was placed on the shoulder. The possibility that such a system might mislead by measuring transepithelial potentials was checked. So results from the monocrystant system were compared with simultaneous measurements from a bipolar glass microelectrode (Radiometer EK2802C diameter $4 \mathrm{~mm}$ ) positioned under visual control, so that the two electrodes were adjacent on the airway surface. No significant difference was observed ( $\Delta$ pH (glassantimony) $+0.15 \pm 0.24(6), p>0.1)$. In other pilot studies it was also shown that the results were influenced neither by $4 \%$ topical lignocaine instillation into the larynx nor by electrical leakage from endoscopic equipment. We found that the endobronchial and endotracheal $\mathrm{pH}$ were acidic. Five patients without pulmonary disease showed: tracheal pH 5.71 (SEM 0.29), right main bronchus $5.62(0 \cdot 18)$, left main bronchus $5.60(0.25)$. These results were not significantly different. Also the main bronchi did not differ from the lobar bronchi. In 21 patients with COAD similar values (which did not vary within the airways) were found: tracheal $5.76(0.33)$ RMB 5.65 (0.50), LMB 5.60 (0.32). In patients with pneumonia no difference could be detected between the bronchi of affected and unaffected lobes. We also studied the $\mathrm{pH}$ of peripheral airways by "wedging" the antimony microelectrode. Results were significantly less acid than the central airways (for example, left lower lobe bronchus 5.34 $(0.11)(5)$, LLL wedge $6.87(0.27)(5)$, right lower lobe bronchus $5.60(0.49)(13)$, RLL wedge $6.89(0.64)(13)$.

Slowing of sternomastoid twitch maximum relaxation rate with inspiratory loaded breathing

VHF MAK, SG SPIRO Rayne Institute, University College Hospital, London We have previously reported slowing of the sternomastoid muscle twitch maximum relaxation rate (TMRR) with fatigue induced by headlift exercise which correlated well with the 20:50 ratio (Mak and Spiro. BTS proceedings, Thorax 1989;44:887P). However, the role of sternomastoid fatigue in respiratory failure is unclear, so we set out to determine if sternomastoid fatigue could be detected when the respiratory muscle system is subjected to a heavy work load. Five normal subjects (mean age 24.6 ) were tested. The techniques used to measure TMRR were as described previously. Fresh state twitches were measured and then the subjects were sat up to perform maximal inspiratory efforts against a fixed inspiratory resistance. Expiration was unloaded and the inspiratory effort was measured on a Bourdon type vacuum gauge. The subjects had several practice attempts before their maximal inspiratory pressure (PImax) was determined. The subjects were then asked to make maximal inspiratory efforts sustained for two seconds every four to five seconds until they could not achieve $70 \%$ of their initial PImax on three successive attempts (inspiratory loaded breathing, ILB). The sternomastoid TMRR was then determined at one minute intervals following ILB for 10 minutes. The mean fresh state TMRR was $8.47 \%$ force loss $/ 10$ ms (range 7.27-9.86) and the mean PImax was $118 \mathrm{~cm} \mathrm{H}_{2} \mathrm{O}$ (range 90-150). The mean duration of ILB was 8.33 min (range $6-12 \cdot 5$ ). The mean fall in the TMRR one min post ILB was $67.2 \%$ of fresh state (SD 9.4) and the maximum fall occurred at two minutes $(66.2 \%$ SD $7 \cdot 3)$. At five minutes the TMRR had recovered to $78.4 \%$ (SD $12 \cdot 2$ ) and at 10 minutes to $87 \cdot 2 \%$ (SD 13.4). We conclude that the sternomastoid TMRR can detect fatigue induced by heavy respiratory loads and therefore may be used to detect fatigue of the accessory muscles in breathless patients.

We thank the Chest, Heart, and Stroke Association for financial support.

Aerosol delivery during mechanical ventilation: nebuliser or nebuhaler?

AK SIMONDS, SP NEWMAN, D COX, SW CLARKE Department of Thoracic Medicine and Intensive Care Unit, Royal Free Hospital, London Aerosol therapy during mechanical ventilation is poorly standardised and often inefficient. Nebulised bronchodilators are used commonly in ventilated patients and it has been suggested that drug delivery from a metered dose inhaler (MDI) via a spacer device is superior to wet nebulisation during mechanical ventilation (Crit Care Med 1989;17:S153). Using a Servo $900 B$ ventilator (MV 10 litres, $\mathrm{R}$ 15/min, I:E ratio $1: 2$ ) connected through an $8.0 \mathrm{~mm}$ endotracheal tube (ETT) to a model lung system, we have compared efficiency of delivery of bronchodilator from a standard nebuliser and $\mathrm{MDI} /$ Nebuhaler combination, each situated between the ventilator circuit and catheter mount. Five milligrams of salbutamol labelled with $T c 99 \mathrm{~m}$ in $4 \mathrm{ml}$ saline was nebulised to dryness over 15 minutes in a Cirrus nebuliser (Intersurgical), driven by a CR-60 compressor (Medic-Aid). For the spacer comparison four puffs of $\mathrm{Tc} 99 \mathrm{~m}$ labelled salbutamol were inserted into a Nebuhaler which was placed in the ventilator circuit for approximately one minute. During the period of incorporation of the nebuhaler into the circuit, minute volume was increased to 20 litres to compensate for increased dead space. Radiolabelled drug was collected on a Pall Ultipor filter situated between the ETT and model lung and counts corrected for background activity. Mean (SD) delivery as a $\%$ of initial dose was $11.04(0.93)$ from the nebuliser and $1.77(0.29)$ from the $\mathrm{MDI} /$ Nebuhaler. Despite the advantages of reducing precipitation and duration of treatment, the $\mathrm{MDI} / \mathrm{Nebuhaler}$ system is less efficient than a conventional nebuliser and temporarily increases the dead space and compressible gas volume of the ventilator circuit, which may be detrimental.

Transbronchial lung biopsy (TBB) appearances before and after treatment for acute lung rejection (AR) in heartlung transplants (HLT)

CA CLELLAND, S STEWART, TW HIGENBOTTAM, JP SCOTT, J WALLWORK Heart-Lung Transplant Research Unit, Papworth Hospital, Cambridge TBB is increasingly being used to differentiate AR from pulmonary infection in HLTs as other methods are less sensitive and less specific. The characteristic histological feature of AR is dense perivascular lymphocytic infiltration. Some HLT patients suffer repeated episodes of AR therefore it is important to be able to distinguish treated rejection (TR) from AR. To aid the interpretation of serial TBBs we have documented the histological features of 28 biopsy specimens taken during AR before institution of treatment and their subsequent paired follow up biopsy specimens after a course of augmented immunosuppression (mean interval between initial and follow-up biopsies was 23.5 days, SD 13.4 days). Infection was strictly excluded by histology and culture of bronchoalveolar lavage and sputum. The frequency of perivascular infiltrates and the presence of lymphocytes, plasma cells, neutrophils and eosinophils in the inflammatory infiltrates were assessed semi-quantitatively as was haemosiderin deposition (-, + , or ++ allocated for each cell type). Lymphocytes before treatment were generally large and blast like and were more numerous than in the follow up biopsies where morphologically there was a change towards smaller, widely spaced lymphocytes. The frequency and size of infiltrates also decreased with treatment (mean size $7 \cdot 1$ (SD 4.8) cell thickness in $A R$ and 3.8 (3.5) after treatment). The lack of plasma cells points towards a cell mediated response in AR. Neutrophils and eosinophils were less numerous after treatment but haemosiderin deposition was increased. The changes in cellular infiltrates of bronchioles paralleled those of the perivascular infiltrates. We conclude analysis of the quantitative and morphological changes in follow up TBB specimens offers some assistance in determining the efficacy of treatment. In addition, two episodes of infection were diagnosed from follow up biopsies.

\begin{tabular}{lllllllll} 
& \multicolumn{2}{l}{ Rejection (\%) } & & \multicolumn{3}{c}{ Treated rejection (\%) } \\
\cline { 2 - 4 } \cline { 7 - 9 } Cell type & - & + & ++ & & & + & ++ \\
\hline Lymphocytes & 11 & 46 & 43 & & 29 & 68 & 3 \\
Plasma cells & 89 & 11 & 0 & & 89 & 11 & 0 \\
Neutrophils & 50 & 39 & 11 & & 68 & 25 & 7 \\
Eosinophils & 57 & 36 & 7 & & 89 & 4 & 7 \\
Haemosiderin & 54 & 32 & 14 & & 39 & 36 & 25 \\
\hline
\end{tabular}


Survival and quality of life following heart-lung transplantation in Eisenmenger's syndrome

G CREMONA, JP SCOTT, N CAINE, L SHARPLES, AT DINH XUAN, TW HIGENBOTTAM, J WALIWORK Papworth Hospital, Cambridge Patients with end stage Eisenmenger's syndrome (ES) represent the largest disease group undergoing combined heart and lung transplantation (HLT) at Papworth Hospital (29.85\%). Although HLT represents the only potentially curative therapy for ES, the selection and timing of ES patients is often difficult as the patients have usually been chronically ill for a long time and with recent deterioration over several years. Between March 1982 and July 1989,42 patients, mean age 29.8 (range 9-50) y, were assessed and accepted for HLT. Twenty of them, mean age 29 (range 14-43) $y$, underwent HLT. All the transplanted patients had severe long standing pulmonary hypertension (mean PAP $79.70 \mathrm{~mm} \mathrm{Hg}$ ) secondary to congenital heart defects and severe functional limitation (classes III/IV; mixed venous oxygen saturation: $73 \%$ ). At the time of last follow up 15 patients have survived. One patient died of cerebrovascular accident 12 days post operation (PO); two patients died of CMV infection at 46 and 85 days PO. One patient died of infection at 9 days PO. Only one patient died of chronic rejection (416 days PO). Two patients have obliterative bronchiolitis. The actuarial probability of survival at one year is $78.95 \%$ (SEM 9.35). Mean rejection rate is 1.32 episodes per patient per year. The Nottingham Health Profile was applied to eight of these patients to assess quality of life before and after HLT. A significant $(p<0.05)$ improvement was found in all areas explored by the profile except for sleep. These results indicate that HLT is a suitable treatment for end stage ES and offers a very significant improvement in quality of life.

Pulmonary function as a predictor of pneumonitis in bone marrow transplant recipients

HJ MILBURN, HG PRENTICE, RM DU BOIS Royal Free Hospital, London If the high mortality associated with pneumonitis following bone marrow transplantation (BMT) is to be reduced, a diagnosis must be made early. In the initial stages of pneumonitis, the patient may have no abnormal clinical signs and a normal chest radiograph leading to a reluctance to investigate at this stage. We have measured pulmonary function (PF) in BMT recipients to attempt to (1) identify those patients at risk of developing pneumonitis, and (2) determine whether changes in PF are good early indicators of pneumonitis. PF was measured before and at intervals after BMT in 39 patients. Fifteen of these later developed pneumonitis and PF was measured when symptoms first developed. Before BMT there was no difference in PF between patients who later developed pneumonitis and those who did not. Six weeks post BMT the carbon monoxide gas transfer (TLCO) was 71 (SEM 11) $\%$ of the pretransplant value in those who went on to develop pneumonitis and 109 (38) $\%$ in patients who did not $(p<0.01)$. These measurements three months after BMT were $77(7) \%$ and $96(26) \%$ respectively (p < $0.05)$. There was no difference between the two groups of patients for $\mathrm{FEV}_{1}, \mathrm{FVC}$, or lung volumes six weeks and three months after BMT. In patients developing pneumo- nitis, all parameters fell a further 25-32\% predicted at the onset of symptoms, even in those with no abnormal signs and a normal radiograph. These results suggest that (1) pulmonary function measurements are good indicators of developing pneumonitis after BMT, and (2) a significant fall in TLCo six weeks and/or three months after BMT compared with pretransplant levels predicts those patients at risk of developing pneumonitis. This further suggests that pneumonitis develops on a background of lung damage presumably sustained at or shortly after transplantation.

Value of pulmonary function testing in the diagnosis of lung rejection or infection following lung transplantation

P NEILL, AD GASCOIGNE, TN STONE, JH DARK, GJ GIBSON, PA CORRIS Departments of Respiratory Medicine and Cardiothoracic Surgery, Freeman Hospital, Newcastle upon Tyne The most important clinical problems following successful single lung or heart lung transplantation lie in the prompt recognition of opportunistic pneumonia and pulmonary rejection. Symptoms and signs, if any, are non-specific and the chest radiograph commonly shows no abnormality. Penketh et al (Thorax 1988;43:762) suggested that spirometric measurements were helpful in this situation but they reported only inconsistent changes in gas transfer. We have evaluated retrospectively the predictive value of changes in $\mathrm{FEV}_{1}$ and carbon monoxide transfer factor ( $\mathrm{TLCO}_{\mathrm{L}}$ ) performed prior to 82 transbronchial lung biopsy and lavage procedures in 18 lung or heart lung transplant recipients at Freeman Hospital. On 63 occasions the patients were investigated because of clinical deterioration and in the remaining 19 instances the only indication was a sustained decline in lung function. In the former group rejection or infection was proved on 51 of 63 occasions. In the prediction of these changes a fall in FEV $>10 \%$ below the previous baseline showed a sensitivity of $80 \%$ and specificity of $75 \%$; the corresponding sensitivity and specificity of a fall in TLCo $>10 \%$ were $86 \%$ and $66 \%$ respectively. When the indication for biopsy was a decline in function alone, a positive biopsy and/or lavage was obtained on 12 of 19 occasions. In these cases the positive predictive value of a $10 \%$ decline in function was $72 \%$ for $\mathrm{FEV}_{1}$ and $69 \%$ for TLco. We conclude that deteriorations in $\mathrm{FEV}_{1}$ or TLCO are equally useful as guides to infection or rejection of transplanted lungs.

Heart-lung transplantation for children with cystic fibrosis

B WHITEHEAD, $P$ HELMS, $M$ GOODWIN, I MARTIN, JP SCOTT, RL SMYTH, TW HIGENBOTTAM, J WALLWORK, M ELLIOTT, M DE LEVAL Hospitals for Sick Children, Great Ormond Street, London, and Papworth Hospital, Papworth Everard, Cambridge Children with cystic fibrosis (CF) remain the largest group of patients $(67 \%)$ referred to the Hospital for Sick Children for heart-lung transplantation (HLT). Between March 1988 and September 1989,24 have been assessed, of whom 17 $(70 \%)$ were accepted on to an active transplant list. Eight have received transplants (age range 5-14 years) while five have died awaiting suitable organs. Donor and recipient were matched by ABO blood group, lung size and cytomegalovirus antibody status. Initial quadruple immunosuppression (cyclosporin A, Azathioprine, methylprednisolone, and antithymocyte globulin) was used reducing to dual or triple therapy. There were six survivors (8-440 days). The two deaths occurred respectively at 36 hours from donor organ failure and 69 days from severe pulmonary infection. Surveillance and grading of rejection was made histologically from transbronchial biopsy specimens obtained through a rigid or fibreoptic bronchoscope. There was a mean incidence of four episodes of rejection in the first six post-operative months. Other complications included: pulmonary infection (total number $=18$ ) of which Pseudomonas aeruginosa was the most common pathogen; diabetes mellitus $(n=3)$; meconium ileus equivalent $(n=3)$ and pancreatitis $(n=1)$. All survivors experienced sustained improvement in quality of life and in dynamic lung function. Postoperative mean $\mathrm{FEV}_{1}$ was $68 \%$ of predicted normal, compared with a mean of $21 \%$ before transplantation.

Vasodilatory properties of prostacyclin aid lung preservation

DK JONES, DW MULVIN, M JOHNSTON University of Colorado Health Sciences Centre, Denver, Colorado, USA Using an isolated rat lung model, we have investigated preservation solutions used in clinical heart-lung transplantation. Prior to the removal of heart and lungs from anaesthetised rats, the lungs were flushed with $50 \mathrm{ml}$ solutions at $4^{\circ} \mathrm{C}$ and then kept in cold saline for 6 hours. They were then reperfused with blood at $37^{\circ} \mathrm{C}$ for 30 minutes. Weight gain and wet to dry lung weight ratios were used as indices of lung injury. We found that an extracellular solution containing blood, albumin, mannitol and prostacyclin (Wallwork's solution: Transplantation 1987;44:654) gave significantly better protection ( $p<0.05$, ANOVA) than an intracellular solution, an extracellular solution with low potassium plus dextran, or a no flush technique. We then compared standard Wallwork's solution (WS) to Wallwork's solution without prostacyclin (WS - PG12) and Wallwork's solution without prostacyclin but with $5 \mathrm{mg}$ and $10 \mathrm{mg}$ of glyceryl trinitrate (WS + 5GTN and WS + 10GTN). Results (mean (SD)) were compared with ANOVA $\left({ }^{\star}=p<0.05\right.$ compared with WS - PG12). These results suggest that the rat model is a simple and efficient method for screening preservation solutions, that Wallwork's solution has advantages over others in lung preservation, and that the addition of prostacyclin confers benefit but that a conventional vasodilator may work just as well.

\begin{tabular}{lll}
\hline & Wt gain $(g)$ & Wet/drywt \\
\hline WS & $1 \cdot 6(0 \cdot 1)^{\star}$ & $6 \cdot 3(0 \cdot 1)^{\star}$ \\
WS - PG12 & $2 \cdot 8(0.2)$ & $8 \cdot 1(0 \cdot 7)$ \\
WS + 5GTN & $2 \cdot 2(0.4)$ & $7 \cdot 1(0 \cdot 3)$ \\
WS + 10GTN & $1.9(0.2)^{\star}$ & $6 \cdot 7(0 \cdot 3)^{\star}$ \\
\hline
\end{tabular}

Role of viral culture in the diagnosis of pneumonia after heart-lung transplantation

JP SCOTT, TW HIGENBOTTAM, G FRADET, RL SMYTH, C CLELLAND, E SOLIS, J WALLWORK Heart-lung Transplant Research Unit, Papworth Hospital, Cambridge Opportunist viral pneumonia has long been a major cause of morbidity and mortality after transplanta- 
tion (Dummer et al. $J$ Infect Dis 1987;155:202). In heart-lung transplant (HLT) recipients most cases have involved infections with cytomegalovirus (CMV) or herpes simplex virus (HSV). We have previously reported the value of transbronchial biopsies both in infection and rejection in these patients (Higenbottam et al. Transplantation 1988;46:532). We have taken serially lung biopsy and bronchoalveolar lavage material for viral culture and transbronchial biopsy tissue for histology on 126 occasions on 42 HLT recipients since November 1988. CMV was cultured from lavage and/or biopsy specimens on 28 occasions in 16 sero positive patients. Lavage specimens more commonly gave positive results, lung biopsy having $65 \%$ of the sensitivity of lavage specimens, but both were required. Of the 14 cases of histology confirmed CMV reactivation, in only five $(36 \%)$ was viral culture positive whereas it was positive in $31 \%$ of the cases histology confirmed lung rejection. Of the CMV positive lung cultures only three out of 27 have preceded, within six months, a need for clinical treatment with ganciclovir. By comparison with five positive cultures for HSV four $(80 \%)$ were followed within two weeks by treatment with intravenous acyclovir for HSV pneumonitis. In contrast to transbronchial biopsy histology, we conclude that CMV lung and lavage culture is of little value, whereas positive culture of $\mathrm{HSV}$ is frequently so. This may reflect the chronic CMV infection of our patients.

\section{Which predicted values for transplan- ted lungs?}

BA OTULANA, KD MACKENZIE, BA MIST, I SHARPLES, TW HIGENBOTTAM, J WALLWORK Papworth Hospital, Cambridge Following lung transplantation pulmonary function tests are commonly expressed as per cent predicted based on the recipient characteristics. Although convenient, no scientific proof has been advanced to support the use of recipient characteristics in preference to the donor's. The transplanted lung retains normal elastic properties (Glanville et al. Am Rev Respir Dis 1988;137:308) but the host thoracic cage determines the ultimate size of the lungs (Otulana et al. Transplantation (in press)). We have studied $\mathrm{FEV}_{1}, \mathrm{FVC}, \mathrm{PEF}$, $T_{L C O}, \mathrm{KCO}, \mathrm{RV}$, and TLC in 17 (11 males), mean age $28 \cdot 1 \mathrm{y}$, longest surviving recipients of heart-lung transplantation (HLT). Donors were selected on the basis of radiological matching as previously described (Hakim et al. J Thorac Cardiovasc Surg 1988;95:474). The PFT measurements were taken before transplantation and then at one, two, and six months and thereafter every three months, periods of lung rejection and infection and patients with obliterative bronchiolitis being excluded. Multiple regression analyses were carried out with the time related measurements as the dependent variable and both the predicted recipient and predicted donor values as independent variables (table) A close relationship is shown by a high and significant $(p<0.05)$ regression coefficient. FVC (and PEF) relates more to the recipient predicted values before transplantation, and again from nine months after transplantation onwards (the $F E V_{1}$ did not assume recipient characteristics until one year). TLco (and Kco) did not show a consistently significant relationship with either predicted value. TLC (and RV) progressively assumes recipient characteristics from immediately after transplantation. These preliminary findings may provide a basis for reference values in lung transplant patients.

Bronchoalveolar lavage cell counts during acute rejection and infection following lung transplantation

HSR HOSKER, P MCARDLE, B SHENTON, J KIRBY, JH DARK, PA CORRIS Departments of Respiratory Medicine and Haematology, Freeman Hospital, Newcastle upon Tyne, and Department of Surgery, University of Newcastle upon Tyne We have performed bronchoalveolar lavage (BAL) on 40 occasions in 12 patients following single lung or heart-lung transplantation as part of a study of lymphocyte activation in rejection. BAL and transbronchial lung biopsy were performed in response to a clinical deterioration suggesting rejection or infection on 31 occasions and as part of routine surveillance on nine occasions. BAL was performed in a subsegment of the right middle lobe or lingula using $180 \mathrm{ml}$ buffered saline. Differential cell counts were made on 300 cells using May-Grünwald-Giemsa stained cytospin preparations. Bacterial or fungal infection was confirmed on 11 , viral infection on seven, and acute rejection on 13 occasions. No evidence of infection or rejection was seen on nine occasions. No significant differences were seen between viral infection and acute rejection but the proportion of lymphocytes was significantly higher in both of these than in normal $(p<0.001)$ or bacterial infection specimens $(p<0.001$ ). Bacterial infection was characterised by a significantly higher neutrophil count than in any other situation. We conclude that BAL \% lymphocyte counts rise in both acute rejection and viral infection following lung transplantation and do not help to distinguish between these two clinical events.

\begin{tabular}{lll}
\hline & \multicolumn{2}{c}{ Mean $(S D)$} \\
\cline { 2 - 3 } Result & $\%$ lymphocytes & \% neutrophils \\
\hline Normal & $5 \cdot 8(3)$ & $5 \cdot 5(2)$ \\
Bacterial infection & $1 \cdot 2(1)$ & $55(27)$ \\
Viral infection & $24(12)$ & $11(6)$ \\
Rejection & $25(18)$ & $9(6)$ \\
\hline
\end{tabular}

Regression coefficients ( $p$ values in parentheses)

\begin{tabular}{|c|c|c|c|c|c|c|c|}
\hline & \\
\hline & & Pre-HLT & 1 & 6 & 12 & 18 & $24 \mathrm{mo}$ \\
\hline FVC & ii & $\begin{array}{l}0.8(0.18) \\
0.1(0.85)\end{array}$ & $\begin{array}{l}0.2(0.46) \\
0.4(0.12)\end{array}$ & $\begin{array}{l}0.4(0.13) \\
0.5(0.03)\end{array}$ & $\begin{array}{l}0.7(0.04) \\
0.4(0.15)\end{array}$ & $\begin{array}{l}0.9(0.01) \\
0.2(0.40)\end{array}$ & $\begin{array}{l}0.8(0.06) \\
0.3(0.18)\end{array}$ \\
\hline TLCo & ii & $\begin{array}{l}1.3(0.05) \\
0.3(0.52)\end{array}$ & $\begin{array}{l}0.7(0.10) \\
0.1(0.75)\end{array}$ & $\begin{array}{l}0.1(0.88) \\
0.7(0.06)\end{array}$ & $\begin{array}{l}0.4(0.26) \\
0.3(0.35)\end{array}$ & $\begin{array}{l}0.4(0.24) \\
0.3(0.28)\end{array}$ & $\begin{array}{l}0.8(0.45) \\
0.3(0.65)\end{array}$ \\
\hline TLC & ii & $\begin{array}{l}1.0(0.07) \\
0.2(0.54)\end{array}$ & $\begin{array}{l}0.7(0.09) \\
0.3(0.34)\end{array}$ & $\begin{array}{l}0.7(0.04) \\
0.6(0.02)\end{array}$ & $\begin{array}{l}0.1(0.01) \\
0.3(0.19)\end{array}$ & $\begin{array}{l}0.8(0.05) \\
0.4(0.15)\end{array}$ & $\begin{array}{l}1.7(0.05) \\
0.3(0.43)\end{array}$ \\
\hline
\end{tabular}

i-recipient predicted; ii-donor predicted.
Detection of malignant cells in pleural fluid using the AgNOR staining method

D BOLDY, JG AYRES, D ROWLANDS, J CROCKER, J YoUNG Departments of Respiratory Medicine and Histopathology, East Birmingham Hospital, Birmingham, and Department of Pathology, University of Birmingham A simple silver staining method to demonstrate nucleolar organiser regions (NORs) showed high AgNOR counts in tumour cells compared to normal tissue (for instance, squamous cell carcinoma of the bronchus $v$ columnar epithelium, mesothelioma $v$ mesothelial cells). In cell imprint preparations, higher AgNOR counts were found in whole cells than in $3 \mu \mathrm{m}$ sections (Boldy $e t$ al. $J$ Pathol 1989;157:75). The clarity of staining suggested that the technique might be usefully applied to cytological preparations eg pleural fluid. Nineteen pleural fluids were examined and slides prepared from each. One slide was stained by the Papanicolaou method in a routine laboratory and reported by an independent pathologist as either malignant or negative. The second slide was stained by the AgNOR method and was classified as probably malignant (many cells with numerous AgNOR dots, without clumping $=++$ ), possibly malignant (few cells with numerous AgNOR dots, no clumping $=+$ ) and negative (no cells with numerous AgNOR dots $=0$ ). The case notes were examined subsequently to determine if the pleural effusion was associated with a malignant course. Good agreement was noted between a positive cytological report and effusion associated with a malignant course $(p=0.0048)$ and between the AgNOR result and clinical diagnosis $(p=0.0048)$. The AgNOR method identified two of three cytology negative, clinically positive cases, but also had three false positive results (two postpneumonic). A prospective study is required to examine more closely whether the AgNOR method may be useful in the diagnosis of malignant pleural effusion.

This work was supported by the Chest, Heart, and Stroke Association.

\begin{tabular}{|c|c|c|c|c|}
\hline & \multicolumn{3}{|c|}{$A g N O R$} & \multirow[b]{2}{*}{$p$} \\
\hline & ++ & + & 0 & \\
\hline Cytology + & $\begin{array}{l}6 \\
3\end{array}$ & $\begin{array}{l}1 \\
2\end{array}$ & $\begin{array}{l}0 \\
7\end{array}$ & 0.023 \\
\hline
\end{tabular}

The AgNOR staining method and prognosis in squamous cell carcinoma of the bronchus

D BOLDY, JG AYRES, D ROWLANDS, J CROCKER, M GILTHORPE, J WATERHOUSE Departments of Respiratory Medicine and Histopathology, East Birmingham Hospital, and West Midlands Regional Cancer Registry, Birmingham Recent work using a silver staining method (AgNOR) to demonstrate nucleolar organiser regions (NORs) has shown that AgNOR counts relate to tumour differentiation in squamous cell carcinoma of the bronchus (SqCCB) and non-Hodgkin's lymphoma. To investigate whether the AgNOR method might be useful as a prognostic indicator, we studied retrospectively 138 patients who underwent surgical resection for a SqCCB at the East Birmingham Hospital in 1977. Pathological T, N and $M$ status were determined by examination of the case notes, operation notes and histo- 
pathology reports and tumours, staged according to the recent IUCC classification. Details of age, sex, site and type of operation, completeness of resection, length of survival and cause of death were also collected. A representative block of tumour tissue was classified histologically and AgNOR counting and DNA flow cytometry were performed with the same paraffin block. Full clinical data were available for all 138 patients. Overall survival, age adjusted, excluding postoperative deaths, was $27.3 \%$ at five years and $20.7 \%$ at 10 years. The five year survival by T status was T1 $36 \%$; T2 $27 \%$; T3 $27 \%$; T4 $10 \%$; by N status NO $40 \%$; N1 $21 \%$; N2 $8 \%$; by stage of disease I $40 \%$; II $22 \%$; IIIa $20 \%$; IIIb $10 \%$; IV $0 \%$. Completeness of resection and operation performed both affected prognosis. Age, DNA ploidy and AgNOR scores were not related to survival, even allowing for stage. of disease. Thus, although the AgNOR counting method may be helpful in the diagnosis of malignancy, it does not appear to be an important prognostic indicator in squamous cell carcinoma of the bronchus.

This work was supported by a grant from the Chest, Heart, and Stroke Association.

DNA ploidy is a prognostic discriminator in early stage, surgically treated, small cell lung cancer

FA CAREY, S PRASAD, D LAMB, CC BIRD Department of Pathology, University of Edinburgh, and Department of Thoracic Surgery, City Hospital, Edinburgh Fifty three surgically resected small cell carcinomas of lung received in Edinburgh University Pathology Department in the years 19827 were analysed for DNA content by flow cytometry. DNA aneuploidy was detected in $41.5 \%$ of the cases while $58.5 \%$ of tumours were either diploid or tetraploid. The relationship of both ploidy and TNM status to two year survival was assessed. Of the patients with diploid/tetraploid tumours, $74.2 \%$ survived for two years or more while only $41 \%$ of the DNA aneuploid group were alive at two years. Nodal status is, however, a better predictor of survival, $72 \%$ of $\mathrm{No} / \mathrm{N} 1$ but only $28.5 \%$ of N2 patients living for two years. When analysis is confined to early stage (No/N1) tumours (39 cases) $20 / 24(83.3 \%$ ) of diploid/tetraploid cases but only $8 / 15$ $(53.3 \%)$ of DNA aneuploid cases were among the survivors. This difference is statistically significant $(p<0.05)$. It is concluded that, while nodal status is the best overall predictor of prognosis in surgically treated small cell lung cancer, DNA ploidy allows for finer prognostic discrimination in early stage tumours.

Stereoisomers of verapamil in drug resistant tumour cell lines

R MILROY, J PLUMB, $S$ BANHAM, $S$ KAYE Department of Respiratory Medicine, Glasgow Royal Infirmary, and Cancer Research Campaign, Department of Medical Oncology, University of Glasgow Verapamil (V), a racemic mixture of the $\mathrm{D}$ and $\mathrm{L}$ isomers (DV and LV) has been used in clinical studies in small cell lung cancer to try and overcome drug resistance (Milroy et al. Lung Cancer 1988;4(suppl):A101). The plasma concentration of $\mathrm{V}$ achievable in the clinic is limited by cardiovascular toxicity. DV is a less potent calcium channel blocker and may be less cardiotoxic than LV (Echizen et al. Am Heart $J$ 1985;109:210). We have studied the resistance modifying activity of both $\mathrm{V}$ and the individual isomers, on the chemosensitivity of the drug resistant tumour cell lines 2780AD, MCF7/AdrR and H69LX10 to doxorubicin (DOX). Neither $\mathrm{V}$, nor the individual isomers had any effect on the drug sensitivity of the parental cell lines (A2780 MCF7, NCI-H69). V increased the sensitivity of all three DOX resistant cell lines. This activity was concentration dependent. The increase in sensitivity to DOX was only 2-3 fold at $2 \mu \mathrm{M}$, the maximum plasma concentration achievable in patients. This activity was maximal at $6-7 \mu \mathrm{M}$, resulting in a 10-12 fold increase in sensitivity for all three cell lines. Both DV and LV were as effective as $\mathrm{V}$ in terms of resistance modifying activity. DV demonstrated the same concentration dependent activity as V. Total cellular DOX accumulation in both 2780AD and MCF7 AdrR was increased 2 fold in the presence of $\mathrm{V}(6.6 \mu \mathrm{M})$. Both DV and LV produced a similar increase in drug accumulation. Use of DV alone in patients could ipcrease the maximum tolerated plasma concentration of verapamil. Thus DV may be a more effective resistance modifier in lung cancer patients. Clinical studies using DV are now under way.

Lung cancer classification: problems of classification based on biopsy and cytological material

D LAMB, E MCGOOGAN (conclusions of working party set up under the auspices of the UKCCR Subcommittee for the Managemen of Lung Cancer and presented on their behalf) Department of Pathology, University of Edinburgh Medical School, Edinburgh The WHO classification of Lung Cancer cannot be applied to small biopsy specimens or to cytological material. Small cell carcinoma is one of the most straightforward diagnoses but even so accuracy is probably no better than $95 \%$. While it is possible to diagnose the better differentiated squamous and adenocarcinomas, it may only be possible to state that the tumour is not of small cell type. Such neoplasms are best described as showing no discernible differentiation but not small cell carcinoma. This description may apply to half the non-small cell carcinomas. We believe that correlation between cytology and histology would be improved if the following categories were used: (1) Squamous cell carcinoma; (2) adenocarcinoma; (3) small cell carcinoma; (4) other specified tumours; (5) no discernible differentiation but not small cell carcinomna; 6 ) no discernible differentiation unclassified carcinoma; (7) not typable for technical reasons. It was decided that the group should attempt to describe criteria for the diagnosis and classification of lung cancer suitable for small biopsies and cytological preparations and audit such a classification with the intention of introducing such a classification on a national basis.

Prospective comparison of disposable and reusable bronchoscopic cytology brushes

WJM KINNEAR, MJ WILKINSON, PD JAMES, IDA JOHNSTON University Hospital, Nottingham We have undertaken a prospective comparison of the two main types of bronchoscopic cytology brushes, reusable bristle and disposable rake, since our impression was that the former yielded superior specimens. Fifty patients undergoing bronchoscopy for suspected malignancy were studied. Samples were taken from each patient with both types of brush in random order. The pathologists' grading of the quality of the specimens (read blind) was similar for both types of brush. A positive diagnosis of malignancy was made in 31 patients with the disposable brush and in 28 patients with the reusable brush ( $p>$ 0.05 ). In six patients the disposable brush gave a positive diagnosis of malignancy but not the reusable bristle, and in three patients the reusable brush was positive but the disposable was negative. The reusable brush gave a positive diagnosis of malignancy in 12 of 25 patients in which it was used first and in 16 of 25 patients in which it was used second (p $>0.05$ ). Prior to this study our usual practice was to use the reusable bristle brush at the end of the bronchoscopy, withdrawing the bronchoscope with the brush still protruding. Of the 25 patients in this study in which this procedure was followed, a positive diagnosis of malignancy was obtained with the reusable brush in 16 patients, whereas the disposable brush specimens taken from the same patients were positive in 19 patients ( $p$ $>0.05$ ). We conclude that the quality of specimens and diagnostic yield from the disposable rake and reusable bristle cytology brushes are similar. The time needed for cleansing the reusable brushes and the risk of transmitting infection may make the disposable rake type the cytology brush of choice, despite the greater cost.

Prevalence of obstructive lung disease in Norwegian communities and the risk associated with occupational titles

P BAKKE, V BASTE, A GULSVIK Department of Thoracic Medicine and Section for Medical Informatics and Statistics, University of Bergen, Norway In a two phased cross sectional survey the prevalence of obstructive lung disease (OLD) was examined in the general population aged 18-73 years of Hordaland county, Norway, in 1988. The first phase was a questionnaire survey of 4992 subjects (response rate $90 \%$ ). In the second phase a stratified sample $(n=1512)$ of those responding in the first phase was invited to a clinical, spirometric, and chest radiographic examination and an occupational history interview covering all jobs held since leaving school. The attendance rate was $84 \%$. The disease rates were compared with those observed in a two phased population survey in Oslo county in 1974, including 1268 subjects aged 20-69 years, using the same diagnostic criteria as the present study. The overall prevalence of OLD was $5.5 \%$ in Oslo 1974 and $7.7 \%$ in Hordaland 1988 ( $p<0.05)$. The mean FEV of those with OLD was $78 \%$ of predicted in Oslo and $74 \%$ in Hordaland $(p>0.05)$. The role of occupational title as a predictor of OLD was examined in a case-control design where the subjects with OLD $(n=103)$ of the Hordaland study were compared with controls $(n=453)$ from a population representative subsample of those attending the second phase of the Hordaland study. Holding a job with high degree or airborne exposure $(\mathrm{OT}++)$ increased the odds for OLD with $6.4(95 \%$ CI 2.3-18.4) compared with holding a job with no airborne exposure (OT - ) after adjustment for sex, age and 
smoking habits. The adjusted odds of OLD in those who had OT ++ jobs and OT + jobs in 1970 was $8.3(95 \%$ CI $2.0-34.4)$ and 1.6 (95\% CI 1.1-2.4) respectively compared with those who had OT - jobs in 1970. These data indicate that the prevalence of OLD is higher in Hordaland county in 1988 than in Oslo county in 1974, and that occupational title is a predictor of OLD.

\section{Protective effect of neonatal BCG may not be detectable by age 14}

CP BREDIN, M GODFREY St Finbarr's and Regional Hospitals, and University College, Cork, Ireland An episode of tuberculosis occurred in a 604 pupil girls suburban secondary school in Cork city in 1986 . The source case was a 14 year old student with smear positive pulmonary tuberculosis (PTB). Five other active cases were detected (two PTB, one pleural, one uterine, one meningitis). Seventy five Heaf positive, radiographic negative pupils were also identified. Routine neonatal BCG was abolished in the city in December 1972. The BCG pupils ( $n=442$, majority age $\geqslant 14$ years) showed no statistical difference in attack rate $(0.94 \%)$ versus the non-BCG (majority age < 14 years) pupils (attack rate $1.09 \%, n=182$ ). In teenage children the waning protective effect of neonatal BCG may reach a non-detectable level by age 14

A comparison of the prevalence of asthma, non-specific bronchial hyperresponsiveness and atopy in Tokelauan children in two environments

J CRANE, TV O'DONNELL, D WAITE, I PRIOR Departments of Medicine and Community Health, Wellington School of Medicine, Wellington, New Zealand We have compared the prevalence of asthma symptoms, non-specific bronchial hyperresponsiveness (BHR), and atopy in 5-15 year old children of Tokelauan migrants living in New Zealand with children living in two of the three remote Pacific atolls that comprise the Tokelau group. Among the younger children (aged 5-9 years) the prevalence of asthma symptoms (NZ 6\% Tokelau 5\%) and BHR (NZ $32 \%$ Tokelau $37 \%$ ) were similar in the two environments. The degree of BHR as measured by geometric mean $\mathrm{PD}_{20}$ was also similar (NZ 2.6, Tokelau $2 \cdot 1 \mu \mathrm{mol} / 1$ ). Atopy as measured by skin weal responses was six times more common among children in New Zealand than in children in Tokelau (NZ 30\%, Tokelau 5\%). Among the older children (10-15 years) asthma symptoms were more prevalent in NZ than Tokelau (NZ $8 \%$, Tokelau 3\%; p = 0.02). BHR was similarly more frequent in New Zealand than in Tokelau (NZ 19\%, Tokelau 11\%; $\mathrm{p}=0.03)$ and was more severe (NZ mean $\mathrm{PD}_{20}$ 3.3, Tokelau $\left.1.6 \mu \mathrm{mol} / \mathrm{l} ; \mathrm{p}=0.04\right)$. Atopy was three times more common in NZ (NZ 33\%, Tokelau 9\%) in this age group. These differences indicate that older but not younger Tokelauan children in New Zealand have more asthma than their atoll peers, associated with a greater frequency and severity of abnormal airway responsiveness. Greater aeroallergen exposure in the New Zealand environment is the most likely cause of these differences.
Vacuum cleaning carpets and airborne levels of Dermatophagoides pteronys sinus

S OWEN, L WALLWORK, R MILLER, J HEPWORTH, R NIVEN, D FISHWICK, S KALRA, A WOODCOCK Wythenshawe Hospital, Manchester House dust mite sensitive asthmatics frequently become symptomatic after vacuum cleaning carpets. This is presumed to be secondary to increased airborne levels of Dermatophagoide pteronyssinus antigen (Der pl) contained in mite faeces (10-40 $\mu \mathrm{m}$ in diameter). We have compared a conventional vacuum cleaner (Hoover Junior) with a new high filtration cleaner (Medivac), which filters $99.99 \%$ of particles down to $0.3 \mu \mathrm{m}$. We sampled air in 16 domestic rooms (Rothero-Mitchell Sampler; Whatman GFF filter; 60 litres per minute for five hours). On the following day rooms were randomised to be vacuumed with either Medivac or Hoover Junior. During vacuuming, and for the next five hours, room air was sampled. Samples were then coded, weighed and analysed blind for Der pl (ELISA). Airborne Der pl levels increased significantly following vacuum cleaning with standard cleaner (control mean (SEM) 1.79 $(0.19) \mathrm{ng}$; after Hoover 7.19 (6.75) ng; $\mathrm{p}<$ $0.01)$. There was no significant change in airborne Der pl after Medivac (control 1.53 (0.65); after Medivac 1.88 (0.79) ng). There was no change in airborne dust weight. Levels of Der pl in carpet dust did not correlate with airborne levels either before or after cleaning. We concluded that Medivac prevents the increase in airborne Der pl levels which follows conventional vacuum cleaning.

\section{Asthma and rhinitis in boiler operators}

AS ROBERTSON, PS BURGE Institute of Occupational Health, University of Birmingham, and East Birmingham Hospital, Birmingham Four boiler operators and one boiler chemist (mean age $47 \cdot 4$, range 35-54 y) from a Birmingham engineering company were referred to outpatients with work related symptoms. All workers complained of acute and chronic respiratory and nasal symptoms. Respiratory symptoms consisted of chest tightness, wheeze, cough and breathlessness and nasal symptoms including blocked or runny nose with pain, crusting and bleeding Their job with the engineering company involved operating and occasionally cleaning oil, coal and gas fired boilers; four were stil employed, one having left one year previously. They had been employed for a mean of 10.6 (range 5-19) years. All had previously been well while working as boiler operators elsewhere (mean $13.8 \mathrm{y}$ ). In November $19844 / 5$ of the workers entered the boiler house after the oil fired boilers had been cleaned by contractors. All were systematically unwell in association with symptoms of asthma and profound nasal irritation that lasted approximately one week. Subsequent to this exposure all workers had variable work related nasal and respiratory symptoms. One worker gave a past history of pneumonia related to boiler cleaning. On examination three had inflamed nasal mucosae and one had a nasal perforation. The mean lung function of those currently work ing was reduced ( $\%$ predicted): $\mathbf{F E V}_{1} \mathbf{7 5} \%$ (SD 18\%), FVC $88 \%$ (SD 11\%). Chest radiographs were normal in all five. Only one had a positive skinprick test response to a common allergen. Serial peak flow recordings in those engaged in routine boiler operations showed occupational asthma in one, small consistent declines in mean peak flow in two, and a normal record in the remaining worker. Although the acute irritant effects of the vanadium contained within oil fired boiler dust is well recognised, the recurrent nasal and respiratory symptoms associated with low level exposure described here have not previously been described.

Establishing threshold values for late asthmatic reactions (LARs) to occupational agents

SC STENTON, JH DENNIS, EH WALTERS, DJ HENDRICK Chest Unit, Newcastle General Hospital, University of Newcastle upon Tyne Inhalation challenge tests are frequently performed in the investigation of occupational asthma but there has been little attempt to establish dose response relationships or threshold levels for the ensuing LARs. The use of a standardised protocol for tests with three different agents has allowed us to determine the thresholds at which LARs first occurred. These comprised the detergent ingredient iso-nonanoyl oxybenzene sulphonate (SINOS) (Thorax 1988;43: 501), the detergent enzyme subtilisin and the antibiotic ceftazidime. Test subjects were exposed workers with symptoms suggestive of occupational asthma. All were challenged with the appropriate agent dissolved in normal saline using a locally designed dosimeter which delivers $50 \mu \mathrm{l}$ of aerosol per dose. Doses were based on environmental measurements in the workplace. The initial dose was chosen to be approximately $1 / 100$ th of the calculated low average cumulative exposure experienced over a normal working shift. Daily increments of $\sqrt{ } 10(3.2)$ fold were used, the challenge sequence finishing when an unequivocal LAR occurred or when the predetermined maximum dose had been administered-generally $10 \times$ the calculated maximum cumulative exposure over a working shift. Saline challenges were interspersed into the challenge sequence in a double blind fashion. $\mathrm{FEV}_{1}$ was used to monitor ventilatory function and a statistical analysis (Thorax 1988;43:866P) was used to determine when LARs had occurred (table).

\begin{tabular}{|c|c|c|c|}
\hline \multicolumn{2}{|c|}{ Subject Agent } & \multirow{2}{*}{$\begin{array}{l}\text { Dose range } \\
0.01-32 \mu \mathrm{g} \\
0.01-100 \mu \mathrm{g} \\
0.01-100 \mu \mathrm{g} \\
0.001-0.1 \mu \mathrm{g}\end{array}$} & \multirow{2}{*}{$\begin{array}{l}L A R \text { threshold } \\
0.032-0.1 \mu \mathrm{g} \\
32-100 \mu \mathrm{g} \\
0.0032-0.01 \mu \mathrm{g} \\
0.01-0.032 \mu \mathrm{g}\end{array}$} \\
\hline & $\begin{array}{l}\text { SINOS } \\
\text { SINOS } \\
\text { SINOS } \\
\text { Subtilisin } \\
\text { Ceftazidime }\end{array}$ & & \\
\hline
\end{tabular}

The extraordinarily wide range encompassed by these thresholds, particularly those for SINOS, indicates the great difficulties which would be encountered in establishing workplace exposure limits once sensitisation has occurred.

Respiratory symptoms in pharmaceutical workers manufacturing opiates

RM AGIUS Institute of Occupational Medicine, Edinburgh Following diagnosis of occupational asthma and rhinitis in a process worker exposed to dust from morphine and other opiates (Agius RM. Br Med J 1989;298:323), a cross sectional survey of 
other employees was carried out using a questionnaire administered by interview opportunistically over a 13 month period. The workers were classified into six occupational groups on the basis of a qualitative assessment of current exposure to opiates. All 112 full time workers who presented responded to the questionnaire. They comprised $69 \%$ of the full time work force at the start of the study. There were no statistically significant differences between the exposure groups in the prevalence of symptoms including the following (overall prevalence in brackets): persistent cough $(14 \%)$, wheeze or dyspnoea $(17 \%)$, persistent itchy nose with sneezing and stuffiness $(21 \%)$ and sneezing not brought on by a cold (56\%) Analysis of the unprompted attribution of symptoms to a particular place, supplemented by temporal relations between symptoms and exposure, showed that 19 workers had one or more of their symptoms in association with opiate exposure. These comprised cough, wheeze or dyspnoea (four), rhinitic symptoms (eight), sneeze (14). Twenty eight reported symptoms, predominantly of sneezing (19) with other occupational exposures known to be associated with such symptoms (the commonest was capsaicin in five). Thirty six reported symptoms in relation to nonoccupational exposures. Analysis of the past history identified commoner or worse previous respiratory symptoms in 43 employees since commencement of this employment. In 18 of them this had been associated with opiate exposure although only seven of them had current symptoms with opiate exposures. Occupational exposure may be associated with symptoms of rhinitis and/or asthma. Further health urveillance and environmental monitoring of these workers is warranted.

Atmospheric air sampling and implication for estimation of antigen exposure in pigeon breeders

K ANDERSON, RAM EWING, G BOYD, G MORRIS Department of Respiratory Medicine, Glasgow Royal Infirmary, and Department of Environmental Health, Strathclyde University, Glasgow Previous reports of factors influencing the development of pigeon breeder's disease have used a calculated estimate of antigen exposure based on the variables of pigeon number and duration of exposure to pigeons with inconsistent conclusions (Fink et al. Chest 1972;62:266; Anderson et al. Thorax 1988;42:798), but no studies have directly measured dust concentrations within lofts to confirm these assumptions. Respirable and non-respirable airborne dust was measured in 21 pigeon lofts, using free standing open face, cyclone, and lapel mounted personal samplers, for comparison with the number of pigeons in the loft and five subjects with pigeon breeder's disease within the group. The number of pigeons (median 40, range 20-100) correlated with total inhalable dust collected over eight hours by the open face sampler (mean $0.51 \mathrm{mg} / \mathrm{m}^{3}$, SEM 0.1 ) when the loft was undisturbed $(R=0.481$, $p=0.041$ ). As expected, dust levels rose after cleaning the loft, doubling on average over the eight hour period. Personal samples demonstrated peaks of exposure to higher levels of dust (respirable: mean $3.59 \mathrm{mg} / \mathrm{m}^{3}$, SEM 0.67 non-respirable: mean $15.25 \mathrm{mg}$ / $\mathrm{m}^{3}$, SEM 4.83), which were independent of the number of pigeons $(R=-0.129$ and
-0.168 respectively; $N_{2} S_{3}$ ). No significant differences in the dust measurements were found between the subjects with and without pigeon breeder's disease. These results explain observed discrepancies between assumed degree of exposure based on the number of pigeons kept and the presence of symptoms reported by others. Dust exposure is not related to pigeon number, which at best relates only to the undisturbed loft, and does not reflect short term peaks of antigen exposure.

Occupational asthma: a surveillance scheme

PFG GANNON, PS BURGE for the Midland Thoracic Society Solihull Hospital, Solihull, West Midlands In January 1989 a surveillance scheme of occupational asthma started in the West Midlands region. Chest physicians and occupational physicians, together with the Health and Safety and Medical Boarding Centre (Pneumoconiosis Panel) doctors, are circulated monthly. For the first year it is looking at both new and old cases, to date 164 cases have been reported. Specific causes of occupational asthma were found in $28 \%$ of cases. The top 10 agents that workers were exposed to include isocyanates $(24 \%)$, colophony $(11 \%)$, flour $(10 \%)$, oil mists $(7 \%)$, epoxy resins $(7 \%)$, wood dusts $(7 \%)$, chrome $(6 \%)$, hard metal $(4 \%)$ humidifiers $(4 \%)$, and zinc $(3 \%)$. In our own unit (129 cases) the diagnosis is made on a history of holiday improvement of respiratory symptoms $(88 \%)$, weekend improvement of symptoms $(78 \%)$, serial peak flow measurement $(72 \%)$, specific IgE antibodies (44\%) and bronchial provocation tests $(8.5 \%)$. At the Medical Boarding Centre (22 cases) the figures are holiday improvement $(86 \%)$, weekend improvement $(95 \%)$, serial peak flow measurement $(50 \%)$, specific IgE antibody $(0 \%)$ and bronchia provocation tests $(5 \%)$. Chest physicians (12 cases) make the diagnosis on holiday improvement $(100 \%)$, weekend improvement $(92 \%)$, serial peak flow $(61 \%)$, specific IgE antibody $(0 \%)$ and bronchial provocation tests $(8 \%)$. Only one case has been reported from the Health and Safety Executive. The outcome of diagnosis was as follows: $32 \%$ were still exposed to the same agent, $27 \%$ were unemployed, $16 \%$ had changed to a new employer, $11 \%$ had been moved within the same employer to avoid exposure, $7 \%$ were off sick, and $6 \%$ had taken early retirement; but in only $2 \%$ had the agent been actually removed from the workplace to prevent further exposure to the individual or their colleagues. This surveillance scheme shows that the diagnosis of occupational asthma is often based on sof data, specific immunology or bronchial challenge testing being used infrequently despite the serious consequences of the diagnosis on the worker's employment.

Correlation between cotton dust levels and the prevalence of byssinosis in Lancashire cotton mills

R NIVEN, D FISHWICK, CAC PICKERING, A FLETCHER Department of Thoracic Medicine, Wythenshawe Hospital, Manchester Nine hundred and fifty-six workers and 407 manmade fibre workers were studied with a respiratory questionnaire to assess the prevalence of byssinosis ( $4.1 \%$ in cotton). Dust levels in the personal breathing zone (PBZ) were estimated for 744 cotton workers using standard (IOM) personal cotton samplers. Current legislation is based on the measurement of work area dust concentrations (WAC) and these were measured in all workrooms. A retrospective dust loading (RDL) was ascribed to each of the 1363 operatives using previous known dust levels over a ten year period for each mill. These three dust parameters (PBZ, WAC, and RDL) were correlated with the percentage of operatives with byssinosis. A significant positive correlation was found with each dust parameter. Pearson's correlation coefficients were: PBZ 0.54, WAC 0.60 and RDL 0.88 . Multiple regression analysis was used to compare RDL with time in the cotton industry, smoking and age. This revealed that only the addition of time in the cotton industry to the equation significantly increased the $\mathbf{R}^{2}$ value. This study suggests that cumulative dust loading is the most important factor associated with the development of byssinosis.

Respiratory symptoms and dust levels in Lancashire weaving mills

SN RAZA, CAC PICKERING, D FISHWICK, A FLETCHER, R NIVEN Department of Thoracic Medicine, Wythenshawe Hospital, Manchester A cross sectional study of 1295 weavers was performed in 15 cotton mills (1202 operatives) and one manmade fibre mill (93). A questionnaire was administered to assess respiratory, nasal and eye symptoms. Dust levels were measured in the personal breathing zone. The mean age was 38 years, 57\% were white and $43 \%$ Asian. The following work related symptom prevalences were found in the cotton mills (manmade fibre mill): Byssinosis $0.33 \%(0 \%)$, productive cough 3\% (3\%), persistent non-productive cough $4 \%(1 \%)$, wheeze $5 \%(6 \%)$, rhinitis $18 \%(8 \%)$ and eye irritation $13 \%(6 \%)$. Only nasal and eye symptoms differed significantly between cotton and manmade fibre. In the cotton weaving mills the mean (SD) dust concentration less fly was $1.63(1.32) \mathrm{mg} / \mathrm{m}^{3}$ and in manmade fibre $0.32(0.24) \mathrm{mg} / \mathrm{m}^{3}$. This difference was highly significant (p $<0.001$ ). The four operatives with byssinosis had a mean age of 43 . The mean dust level in this group was significantly higher at $5.51(5.16) \mathrm{mg} / \mathrm{m}^{3}(\mathrm{p}<0.001)$. This study documents the low prevalence of byssinosis in cotton weaving and recognises the problem of persistent work related nasal and eye irritation.

Occupational asthma due to polyethylene shrink wrapping

PFG GANNON, GFA BENFIELD, PS BURGE Solihull Hospital, Solihull, West Midlands Meat Wrappers asthma due to polyvinyl chloride (PVC) has been described (Soka WN et al. JAMA 1973;226:639). It is believed to be caused by acid anhydrides (from label adhesives) and from pyrolysis products of soybean oil incorporated into the PVC wrapping (Pauli G et al. Clin Allergy 1980;10:263). Occupational asthma due to polyethylene has been described (Skerfving S. Lancet 1980;i:211) but not confirmed by bronchial provocation testing. We have documented occupational asthma in an 18 
year old packer with some pre-existing asthma who worked on a shrink-wrap machine, which briefly heats goods sealed in pure polyethylene packaging to $76^{\circ} \mathrm{C}$ when the wrapping partially shrinks. Two weeks after starting to use this machine he began to get asthmatic attacks six to seven hours after starting work. He had several periods off sick and on the last occasion required hospital admission after 30 minutes at work. The diagnosis was confirmed with serial measurements of peak flow at home and work, and by specific bronchial challenge testing which showed a late asthmatic reaction following an eight minute exposure to polyethylene heated to 76 degrees celsius. Following diagnosis he has been removed from exposure to the shrink-wrap machine with resolution of his symptoms. This case has confirmed polyethylene as a new cause of occupational asthma to be added to the ever expanding list of causes of this preventable condition.

Investigation of the effects of oral administration of vitamin $E$ and $\beta$ carotene on the chemiluminescence responses and the frequency of sister chromatid exchanges in circulating leucocytes from cigarette smokers

R ANDERSON, GA RICHARDS, AJ THERON, CEJ VAN RENSBURG Medical Research Council Unit for the Study of Phagocyte Function, Division of Immunology, Department of Medical Microbiology, Institute for Pathology, University of Pretoria, South Africa Sixty asymptomatic cigarette smokers were randomly allocated into three treatmen groups. Smokers in group 1 received 900 IU of vitamin $E$ (VE) daily for six weeks, while 40 $\mathrm{mg}$ of $\beta$ carotene (BC) daily was administered to those in group 2 for the same period. Individuals in group 3 were treated with a matched placebo. Plasma levels of vitamin $\mathrm{E}$ and $\mathrm{BC}$ as well as circulating leucocyte counts, sister chromatid exchanges (SCEs) and the luminol enhanced chemiluminescence (LECL) responses of blood phagocytes activated with phorbol myristate acetate (PMA) and FMLP with cytochalasin B (FMLP/CB) were measured prior to the administration of the anti-oxidants/placebo after four and six weeks of supplementation and 12 weeks after cessation of treatment. SCEs and leucocyte counts remained unchanged throughout the trial in all three treatment groups. Administration of vitamin $\mathrm{E}$ for four weeks was accompanied by decreased FMLP/CB ( $p<0.005)$ and PMA $(p<0.005)$ activated LECL responses. However, with PMA as stimulant the inhibition of LECL was transient with partial recovery observed after six weeks despite continued administration of vitamin $E$ Administration of $\mathrm{BC}$ was associated with progressive inhibition of both FMLP/CB activated ( $p<0.05$ and $p<0.01$ after four and six weeks respectively) and PMA activated ( $p<0.025$ after six weeks) LECL. No alterations in LECL responses were observed in group 3 (placebo). Vitamin E appeared to inhibit the generation of oxidants by activated phagocytes, while $B C$ scavenged oxidants generated by the myeloperoxidase/ $\mathrm{H}_{2} \mathrm{O}_{2} /$ halide system.
Proteolytic activity and chemotaxis in peripheral blood and pulmonary exudate neutrophils

GM BROWN, DM BROWN, S LANNAN, W MACNEE, $\mathrm{X}$ DONALDSON Institute of Occupational Medicine, and Department of Respiratory Medicine, City Hospital, Edinburgh During pulmonary inflammation neutrophils (PMN) exudate from the vascular space into $t^{\circ}$ tissue. Activation of PMN during this process is thought to cause increased secre tion of proteinases with potential to cause epithelial and connective tissue damage. We therefore measured ${ }^{125}$ I fibronectin proteolysis, epithelial injury and chemotaxin in rat peripheral blood PMN (BPMN) and exudated PMN (EPMN) obtained from the bronchoalveolar space of experimentally inflamed rat lungs. Both populations were around $80 \%$ PMN. The proteolytic activity of the EPMN (5798 (58)), expressed as mean (SEM) cpm degraded ${ }^{125} \mathrm{I}$ fibronectin released, was significantly greater than that of the BPMN (3758 (79); $\mathrm{p}<0.001$ ). At an effector: target cell ratio of $1: 1$ injury to cells of an epithelium like line (A549) caused by EPMN (8014 (398) cpm ${ }^{51} \mathrm{Cr}$ labelled detached cells) was significantly greater than BPMN (5607 (239); p < 0.005). Both the chemotactic and chemokinetic activities of the EPMN were also significantly greater than those of the BPMN: chemotaxis (expresed as mean (SD) migrated cells/high power field) EPMN 92.1 (17.2), BPMN 35.3 (9.0); chemokinesis EPMN $71 \cdot 8$ (17.8), BPMN 4.8 $(1.8) ; p<0.001$. Neutrophils in inflamed lungs have enhanced mobility and increased capacity to damage cellular septal and connective tissue compared with peripheral blood neutrophils.

\section{Research funded by the Colt Foundation.}

\section{Human neutrophil mediated injury to alveolar epithelial cells in vitro}

K DONALDSON, GM BROWN, DM BROWN, W MACNEE Institute of Occupational Medicine, Edinburgh, and Department of Respiratory Medicine, University of Edinburgh Recruitment of neutrophils to the alveolar region of the lung is found in emphysema and in a range of diseases which lead to fibrosis Injury to the alveolar septa may result from the presence of these neutrophils and may be mediated by neutrophil protease or oxidant. We studied the ability of human peripheral blood neutrophils to cause injury to cells of an alveolar epithelial cell line (A549) in vitro. Neutrophils were isolated from the blood of normal volunteers and cocultured with ${ }^{51} \mathrm{Cr}$ labelled alveolar epithelial cells; both lysis and detachment injury were then assessed after four hours of culture. Neutrophils never caused any lysis of the target epithelial cells either spontaneously or on PMA triggering. However, the neutrophils did cause detachment injury to the epithelial cells without PMA (epithelial detachment injury in mean (SD) $\mathrm{cpm}$ of ${ }^{51} \mathrm{Cr}$ in detached epithelial cells: no neutrophils 749 (745), neutrophils at $1: 1$ effector: target ratio 2294 (502), neutrophils at 5:1 $3808(978)$ ). On addition of PMA there was a considerable increase in detachment injury (detachment injury to epithelial cells expressed as above: no neutrophils + PMA 3661 (330), neutrophils at 5:1 3808 (978), neutrophils at 5:1 + PMA 8546 (415)). The detachment injury could be abolished by including antiprotease in the cocultures detachment injury expressed as above: epithelial cells + neutrophils + PMA 1081 (859), epithelial cells + neutrophils + PMA $+\alpha_{1}$ protease inhibitor 41 (70); antioxidants had no effect on detachment injury. Suitably triggered in inflamed lung, neutrophils may have the potential to injure alveolar epithelial cells.

Research funded by the Colt Foundation.

How might smoke affect neutrophils in the pulmonary microcirculation?

E DROST, C SELBY, C LANNAN, W MACNEE Department of Respiratory Medicine, Rayne Laboratory City Hospital, Edinburgh (During cigarette smoking neutrophils are delayed in the pulmonary circulation (MacNee et al. N Engl J Med (in press)). This delay may occur within the pulmonary capillaries from a smoke induced decrease in cell deformability. This can be measured as an increase in the plateau pressure developed by these cells, after smoke exposure in vitro, when passed at a constan flow across a micropore filter whose size mimics the average dimensions of the human pulmonary capillary segments (MacNee $e t$ al. Am Rev Respir Dis 1989:A298). In order to investigate the mechanism of this smoke induced decrease in neutrophil deformability, we compared the plateau filtration pressure produced by smoke exposed neutrophils from normal subjects with that of neutrophils activated with phorbol myristate acetate (PMA). Although the increase in the plateau filtration pressure was similar in cells exposed for four minutes to vapour phase cigarette smoke (control 4.17 (SEM 2.05) cm $\mathrm{H}_{2} \mathrm{O}$; smoked $15.02(7.03) \mathrm{cm} \mathrm{H}_{2} \mathrm{O}, \mathrm{n}=10$; $\mathrm{p}<0.01$ ) when compared with PMA activated cells (control $5.04(1.64) \mathrm{cm} \mathrm{H}_{2} \mathrm{O}_{\text {; }}$ PMA 9.55 (2.43) $\left.\mathrm{cm} \mathrm{H}_{2} \mathrm{O}, \mathrm{n}=7 ; \mathrm{p}<0.01\right)$, both the spontaneous $(\mathrm{Sp})$ and PMA (P) stimulated release of $\mathrm{H}_{2} \mathrm{O}_{2}$ was lower from smoke exposed cells (Sp 1.4 (0.69), P 7.38 $(1 \cdot 85), \mathrm{n}=5$ ) compared with control cells (Sp 4.25 (2.15); P 21.67 (6.38), n $=5$; p $<0.05$ and $<0.01$ respectively). Furthermore, disruption of the cytoskeleton of the neutrophil by the addition of cytochalasin $B$ $\left(10^{5} \mathrm{M}\right)$ reduced the plateau filtration pressure of smoke exposed cells from $16 \cdot 16$ $(5.64) \mathrm{cm} \mathrm{H}_{2} \mathrm{O}$ to $8.67(4.01) \mathrm{cm} \mathrm{H}_{2} \mathrm{O}(\mathrm{n}=5$; $\mathrm{p}<0.05)$, hence increasing cell deformability. Preliminary results also suggest that the decrease in cell deformability induced by smoke can be reduced by adding an antioxidant. These data suggest that the decrease in neutrophil deformability induced by cigarette smoke does not result in cell activation, but may be an effect of smoke on the cytoskeleton as a result of oxidant induced cell injury.

Supported by the Chest, Heart, and Stroke Association and SHERT.

Effects of phospholipase $C$ in the isolated rat lung: injury, eicosanoid production, and von Willebrand factor antigen release

DK JONES, EM PERRY, LM GRAHAM, NF VOELKEL University of Colorado Health Sciences Center, Denver, USA Phospholipase C 


\begin{tabular}{llll}
\hline & Controls $(n=5)$ & $P L C(n=10)$ & $P L C+$ inhibitors $(n=6)$ \\
\hline$\triangle \mathrm{PAP}(\mathrm{mmHg})$ & $0(0)$ & $25(4)^{\star}$ & $10(3)^{\star \star}$ \\
$\mathrm{Wet} / \mathrm{body} \mathrm{wt}$ & $0.007(0.0004)$ & $0.018(0.001)^{\star}$ & $0.012(0.002)^{\star \star}$ \\
$\mathrm{PGF}_{1}(\mathrm{pg} / \mathrm{ml})$ & $4270(450)$ & $8160(1160)^{\star}$ & $2050(500)$ \\
$\mathrm{TXB}_{2}(\mathrm{pg} / \mathrm{ml})$ & $137(23)$ & $2332(695)^{\star}$ & $221(68)$ \\
$\mathrm{LTC}_{4}(\mathrm{pg} / \mathrm{ml})$ & $290(81)$ & $772(340)$ & $185(24)$ \\
VWF: $\mathrm{Ag}(\mathrm{U} / \mathrm{ml})$ & $0.004(0.001)$ & $0.016(0.002)^{\star}$ & $0.014(0.003)^{\star}$ \\
\hline
\end{tabular}

* Significant difference from controls.

$\star \star$ Significant difference from PLC and controls.

(PLC) is a bacterial exotoxin which may be involved in the pathophysiology of acute pulmonary infections. 1 unit of PLC (or control) was injected into isolated saltperfused rat lungs. Measurements were made of increase in pulmonary artery pressure $(\triangle \mathrm{PAP})$ and lung wet weight to body weight ratios (wet/body wt) as indices of lung injury; von Willebrand factor antigen ( $\mathrm{WW}: \mathrm{Ag})$ as an index of endothelial cell damage; and perfusate levels of 6-keto-PGF ${ }_{1 \alpha}\left(\mathbf{P G F}_{1 \alpha}\right)$, thromboxane $\mathrm{B}_{2}\left(\mathrm{TXB}_{2}\right)$ and leukotriene $\mathrm{C}_{4}\left(\mathrm{LTC}_{4}\right)$ as potential mediators. A combination of a cyclo-oxygenase inhibitor and a lipo-oxygenase inhibitor was administered prior to PLC in a further experiment. Results are given in the table as mean (SEM); differences between means were significant at $\mathrm{p}<0.05$ with ANOVA.

These results show that PLC is capable of injuring the pulmonary endothelium directly and that blockade of eicosanoid production only partially ameliorates the lung injury.

Relation between bronchoalveolar lavage (BAL) cell profiles and airway reactivity to histamine after smoke inhalation injury

J KINSELLA, R CARTER, CG GEMMELL, WH REID, CJ CLARK Departments of Respiratory Medicine, Hairmyres Hospital and Royal Infirmary, Glasgow In a study of the number and activity of inflammatory cells in BAL fluid in asthma and their relation to airways reactivity (Kelly et al. Thorax 1988;43:684 a correlation was found between reactivity and both neutrophil numbers and macrophage activity. Acute smoke inhalation is very frequently associated with increased airways reactivity (Kinsella et al. BTS proceedings, Thorax 1989;44:362P). This study investigates the relationship between BAL fluid cell profiles and airways reactivity in a group of 13 patients following acute smoke inhalation injury. BAL was performed within 24 hours of injury and lung function assessment within one week. One patient had normal reactivity (histamine $\mathrm{PC}_{20}>8 \mathrm{mg}$ / $\mathrm{ml}$ ), eight had mildly increased reactivity (histamine $\mathrm{PC}_{20}$ 1-8 $\mathrm{mg} / \mathrm{ml}$ ) and four had moderately increased reactivity (histamine $\mathrm{PC}_{20} 0 \cdot 125-1 \mathrm{mg} / \mathrm{ml}$. Mean $\mathrm{FEV}_{1}$ was $2 \cdot 46$ litres $(76 \%$ pred). There was a significant negative correlation of histamine $\mathbf{P C}_{20}$ with percentage neutrophil count $(r=0.65$, $p<0.01)$. There were no other significant correlations of absolute, percentage cells counts, chemiluminescence of neutrophils (leucigenin enhanced) or macrophage (luminol enhanced) with increased airways reactivity. This study suggests that neutrophil influx into the airways following acute smoke inhalation may be involved in the pathogenesis of increased airways reactivity.
Efiect of dexamethasone on neutrophil function in vitro and in vivo

DA LOMAS, A CHAMBA, RA STOCKLEY Lung Immunobiochemical Research Laboratory, General Hospital, Birmingham Corticosteroids are potent anti-inflammatory drugs and are widely used in the management of pulmonary disease. Their precise mode of action is unclear but they may exert some of their effects by modulation of neutrophil function. In this study we have demonstrated that dexamethasone causes a, significant $(p<0.025)$ dose related fall in neutrophil chemotaxis in vitro from 53.7 (SEM 9.6) to $24.7(8.9)$ cells per high power field (cpf) at $10^{-3} \mathrm{~mol} / \mathrm{l}$. However, dexamethasone had no effect on spontaneous superoxide production in vitro. Six healthy volunteers then took 12 $\mathrm{mg}$ of dexamethasone daily for three consecutive days and neutrophil function was measured for two days prior to therapy, during therapy and one week after treatment. Dexamethasone levels were measured during oral therapy for comparison with neutrophil function. The serum concentrations were $1.26(0.28) \times 10^{-7} \mathrm{~mol} / 1$ on day $1,1.44(0.15)$ $\times 10^{-7} \mathrm{~mol} / \mathrm{l}$ on day 2 and $1.31(0.13) \times 10^{-7}$ $\mathrm{mol} / \mathrm{l}$ on day 3 . Fibronectin digestion fell from $4.3(0.12)$ to $3.24(0.2) \mu \mathrm{g}$ fibronectin/ $10^{5}$ cells during therapy $(\mathrm{p}<0.001$ ), but rose again within one week of cessation of therapy to $3.96(0 \cdot 12)$. A $33^{\circ}$ o change was observed at a plasma concentration $1.44 \times 10^{-7} \mathrm{~mol} / \mathrm{l}$, which was similar to the $30 \%$ change demonstrated previously in vitro at $10^{-7} \mathrm{~mol} / 1$ (Clin Sci 1989;77:35). The average chemotactic response to $10^{-8} \mathrm{M}$ FMLP was $29.5(1.55)$ before therapy and fell $(p<0.025)$ to 13.7 $(1.8) \mathrm{cpf}$ within two hours of taking the first tablets. This was maintained during therapy but rose again to $25 \cdot 75(2.4) \mathrm{cpf}$ one week later. The fall of $46 \%$ was associated with plasma dexamethasone levels of $1.26 \times 10^{-7}$ $\mathrm{mol} / 1$ in vivo compared with a similar fall $\left(46^{\circ}{ }_{0}\right)$ occurring at $10^{-3} \mathrm{~mol} / \mathrm{l}$ in vitro. There was no change in superoxide production $(6 \cdot 13$ $(0.91) \mathrm{nmol} / 10^{5}$ cells/hour prior to therapy and $7 \cdot 77(2 \cdot 17)$ during dexamethasone treatment), confirming the findings of the in vitro experiment.

Effects of extracts of cotton, hemp, and flax dust on human respiratory epi-

A MOGHADDAM, P NICHOLLS, C FELDMAN, RC READ, L-Y HAN, A RUTMAN, H TODD, PJ COLE, R WILsON Host Defence Unit, Department of Thoracic Medicine, National Heart and Lung Institute, London, and Welsh School of Pharmacy, University of Wales, Cardiff Byssinosis occurs among workers exposed to dusts from cotton, flax and soft hemp; fever, bronchoconstriction and hyperreactivity may occur acutely while chronic bronchitis may develop more slowly. The causative agent(s) thelium in vitro may be plant derived, or a product of Gram negative bacterial contamination (for example, lipopolysaccharide). We have examined the effects of aqueous extracts of plant dusts on normal human respiratory epithelium over six hours by measuring ciliary beat frequency (CBF) and examining epithelial structure by light and transmission electron microscopy. The lipopolysaccharide content of the dusts was: cotton $684 \mathrm{ng} / \mathrm{mg}$, flax $240 \mathrm{ng} / \mathrm{mg}$ and hemp $179 \mathrm{ng} / \mathrm{mg}$. All three extracts ( $5 \mathrm{mg} / \mathrm{ml}$ ) slowed $\mathrm{CBF}$ $(p<0.001)$ and caused epithelial disruption in a dose dependent manner. Cotton dust, however, caused only small changes in CBF $(20.5 \%$ ciliary slowing $)$ and minor epithelial disruption, while hemp and flax were much more potent $(89.4 \%$ and $53.6 \%$ respectively) Progressive ciliary slowing occurred before epithelial disruption, but dyskinesia was not seen. The activity of hemp extract was heat stable $\left(70^{\circ} \mathrm{C}, 30\right.$ minutes) and most was removed by dialysis. Although gel filtration yielded some activity in the high molecular weight fraction, the majority was in the fraction below $10 \mathrm{kDa}$. We conclude that although the clinical syndrome produced by the three dusts is similar their effects on respiratory epithelium differ. Hemp activity was mainly due to a low molecular weight factor(s), which could be of plant or bacterial origin.

Effect of recombinant cytokines on $\alpha_{1}$ antitrypsin production by monocytes

CA OWEN, RA STOCKLEY Lung Immunobiochemical Research Laboratory, General Hospital, Birmingham Monocytes and macrophages may be an important source of lung $\alpha_{1}$ antitrypsin $\left(\alpha_{1}\right.$ AT). We have shown that only $20 \%$ of monocytes from normal subjects are spontaneously adherent (Clin Sci 1988;75 (suppl 19):45P). We have compared the effects of recombinant cytokines and LPS purified from Haemophilus influenzae (LPS $\mathrm{HI}$ ) on $\alpha_{1}$ AT production by adherent and non-adherent monocytes. Monocytes were isolated from six normal subjects, the adherent and non adherent subpopulations were separated and cultured for 24 hours with and without interferon gamma (IF, $1000 \mathrm{U}$ $\mathrm{ml}$ ), tumour necrosis factor (TNF, $1000 \mathrm{U} /$ $\mathrm{ml})$, granulocyte macrophage colony stimulating factor (GMCSF, $50 \mathrm{U} / \mathrm{ml}$ ), and LPS HI $(1 \mu \mathrm{g} / \mathrm{ml})$. The average baseline cellular content of the non adherent monocytes (81 (SEM 16) ng/million cells) was greater $(p<0.025)$ than that of the adheren monocytes $(22.9(2.8) ; p<0.025)$. The control adherent cells synthesised $\alpha_{1}$ AT over 24 hours in culture, increasing to $79 \cdot 7(15 \cdot 7)$ $(p<0.025)$ but increased more when treated with all of the cytokines tested (IF 108.6 (17.3); LPS HI 98.9 (17.3) (p < 0.025) and TNF $96.2(14.6)(p<0.05)$. The control non-adherent monocytes also synthesised $\alpha_{1}$ AT in culture, increasing to $149.2(21.5)$ $(\mathrm{p}<0.025)$ but this production was not increased by IF, TNF or LPS HI. GMCSF was the only cytokine which increased $\alpha_{1}$ AT production by both the adherent (control $69 \cdot 1$ (14.8) and GMCSF 96.9 (17.3); p < 0.025) and non-adherent monocytes (control 206.5 (51.3) and GMCSF 228.8 (50.7); p < 0.05). The results indicate that upregulation of $\alpha$ AT production by a variety of cytokines is generally a feature of a subset of monocytes from normal subjects. 
Endothelin (ET), a potent vasoconstrictor, is also a growth factor and chemoattractant for fibroblasts (FB)

PEACOCK AJ, SHOCK A, GRAY AJ, REEVES JT, LAURENT JG Medicine 1, Southampton General Hospital, and Biochemistry Unit, Department of Thoracic Medicine, National Heart and Lung Institute, London Pulmonary hypertension (PHT) from whatever cause is accompanied by pulmonary vascular remodelling and hypertrophy of the pulmonary arteries. This involves deposition of both contractile and connective tissue elements in media and adventitia respectively, but the mechanism of the hypertrophy is unknown. We hypothesised that the same mediator might cause both the vasoconstriction and the hypertrophy. ET is a novel peptide secreted by vascular endothelial cells which is known to be a vasoconstrictor in both the systemic and pulmonary circulations. We tested the effect of ET on the chemotaxis and growth of rat skin FB. Chemotaxis: FB were placed in the upper wells of modified Boyden chambers, separated by filters from lower wells containing $\mathrm{ET}$ in diluting concentrations. After five hours the FB that passed through the filter were counted. ET stimulated chemotaxis occurred over a range of concentrations but was maximal at $3.25 \times 10^{-10} \mathrm{M}(19.0$ (SEM $1 \cdot 1)$ cells/field $v 4.0(1.6)$ field for control; $\mathrm{p}<0.001)$. Growth: FB were grown in 96 well plates in the presence of ET at varying concentrations and, after seven days, cells were counted by measuring light absorbance after staining with methylene blue. ET stimulated growth occurred over a range of concentrations but was maximal at $2.5 \times 10^{-5}$ M $(121 \%(9 \%)$ stimulation relative to control; $\mathrm{p}<0.01)$. We conclude that ET causes chemotaxis and growth of FB but that growth requires higher concentrations of the peptide. These data support the hypothesis that a single endothelial cell product may play a part in both the initial vasoconstriction and the subsequent hypertrophy which occurs in PHT.

Pyocyanin and 1-hydroxyphenazine interact pro-oxidatively with human neutrophils in vitro

GJ RAS, R ANDERSON, GW TAYLOR, R WILSON, PJ COLE MRC Unit for Phagocyte Studies, University of Pretoria, South Africa; Department of Clinical Pharmacology, Royal Postgraduate Medical School, London; and Host Defence Unit, National Heart and Lung Institute, London The effects of the Pseudomonas aeruginosa derived pigments pyocyanin and 1-hydroxyphenazine (1-hp) on the spontaneous and stimulus activated membrane associated oxidative metabolism responses and on the release of lysosomal enzymes by human neutrophils were investigated in vitro. Pyocyanin but not 1-hp increased both the spontaneous and stimulated (F-met-leu-phe, opsonised zymosan, calcium ionophore and phorbolmyristate-acetate) generation of superoxide by neutrophils ( $<<0.05$ ), with maximal effects observed at a $25 \mu \mathrm{M}$ concentration of the pigment. Pyocyanin also increased the rate and duration of oxygen uptake by activated neutrophils $(p<0.05)$. Both agents, but especially $1-\mathrm{hp}$, increased the myeloperoxidase (MPO) mediated iodinating activity of neutrophils ( $p<0.05$ ) which, in the case of 1-hp, was due to stimulation of the release of MPO by activated neutrophils ( $p<$ 0.05 ). In comparison with $1-\mathrm{hp}$, pyocyanin caused only slight enhancement of the release of MPO and lysozyme by stimulated neutrophils, but was found to be more potent with respect to the release of the specific granule marker, vitamin B-12 binding protein. These data demonstrate diverse, proinflammatory interactions of pyocyanin and 1-hp with human phagocytes, which may intensify neutrophil mediated tissue damage during $P$ aeruginosa infections.

In vitro neutrophil "filterability" determines in vivo neutrophil retention in the lungs in man

C SELBY, E DROST, PK WRAITH, GDO LOWE, W MACNEE Departments of Respiratory Medicine, City Hospital, Edinburgh, and Medicine, Royal Infirmary, Glasgow In a gamma camera computer system to follow the reinjection of indium-111 neutrophils (PMN) and technetium-99m erythrocytes (RBC), 11.3 (SEM 3.2\%) of injected PMN in normal subjects are retained in their first passage through the pulmonary circulation (FPR) relative to the passage of RBC. Subsequently, PMN washout from the lungs exponentially. We have shown in both young and elderly normal individuals that regional $P M N$ lung sequestration $10 \mathrm{~min}$ after reinjection (10'S) correlates with local blood velocity measured as RBC lung transit time (TT) (MacNee et al. $N$ Engl J Med (in press); Selby et al. Thorax 1989;44:874P. This relationship is also present five minutes after reinjection $(\mathrm{r}=0.82, \mathrm{p}<0.001)$, suggesting that haemodynamic factors are important in influencing this sequestration. However, FPR does not correlate with RBC TT $(\mathrm{r}=0.09 ; \mathrm{p}=0.7)$ or with $\mathrm{PMN} 10$ 'S $(\mathrm{r}=0.33$; $p=0 \cdot 2)$. In an attempt to model the passage of PMN (diam $7 \mu \mathrm{m}$ ) through the lung capillaries (diam $5 \mu \mathrm{m}$ ), we have measured the plateau pressure that develops when $10^{5}$ PMN ml ${ }^{-1}$ pass at constant flow through a micropore filter (pore diam $5 \mu \mathrm{m}$ ). Aliquots of harvested PMN, from 6 subjects $(3 \mathrm{M}, 3 \mathrm{~F}$; 75-87 y) undergoing eight in vivo measurements of lung kinetics, were taken just prior to radiolabelling. Immediately after reinjection of the radiolabelled cells, the aliquot was filtered. The plateau pressure developed by these cells in vitro correlated significantly with their in vivo lung FPR $(\mathrm{r}=0.89 ; \mathrm{p}=0.003)$ but not with 10 'S $(r=0.47 ; p=0.2)$, nor to rate of washout from the lungs $(r=0.5 ; p=0.2)$. Clearly, neutrophil "filterability" which reflects cell deformability, determines the first pass retention of neutrophils in the lungs as they negotiate the lung microcirculation. By contrast, five to 10 minutes after reinjection, haemodynamic factors are important in neutrophil sequestration, which may reflect cell margination within the larger pulmonary vessels.

N-Acetyl cysteine partially prevents lung fibrosis caused by bleomycin

S SHAHZEIDI, B SARSTRANDT, PK JEFFERY, RJ MCANULTY, GJ LAURENT Biochemistry Unit, Department of Thoracic Medicine, National Heart and Lung Institute, London The $\mathrm{L}$ form of $N$-acetyl cystein (L-NAC) is an acetylated derivative of $\mathrm{L}$-cystein, which is known to have properties of an oxygen radical scavenger. L-NAC has been examined by several investigators in models of lung injury but mostly with histological techniques and its ability to prevent lung fibrosis remains controversial. In this study we assessed the ability of L-NAC to prevent excessive collagen deposition in mice given bleomycin. Mice $\left(B_{6} D_{2} F_{1}\right)$ were given L-NAC in the drinking water ( $1 \%$ solution) for seven days prior to intratracheal administration of bleomycin (150 $\mu \mathrm{g}$ per animal). L-NAC administration was continued and animals were killed after 35 days. Collagen levels were assessed based on hydroxyproline determinations following acid hydrolysis. Total lung collagen content of the animals receiving $L-$ NAC with bleomycin were 2.9 (SD 0.3 ) $\mathrm{mg}$ compared with $3.8(0.5) \mathrm{mg}$ in those receiving bleomycin alone. Lung collagen increased by $41.0 \%(2.3 \%)$ above control in animals receiving $\mathrm{L}-\mathrm{NAC}$ compared with an increase of $84.0 \%(4.7 \%)$ for animals receiving bleomycin alone. The collagen content of the group given bleomycin alone were significantly higher than for animals treated by $\mathrm{L}-$ NAC $(p<0.01)$. These data suggest that $L-$ NAC is partially effective in the prevention of the collagen deposition which follows bleomycin induced lung injury in mice.

Effect of inhaled beclomethasone dipropionate on peripheral neutrophil function in vivo

DC WEIR, S JONES, A CHAMBA, PS BURGE, RA STOCKLEY East Birmingham Hospital and Lung Immunobiochemical Research Laboratory, General Hospital, Birmingham Increased activation and recruitment of peripheral neutrophils (PMN) are thought to play a major role in the pathogenesis of many lung diseases including chronic bronchitis and emphysema. Previous studies have suggested that corticosteroids may reduce PMN chemotaxis and degranulation. We have therefore investigated the effect of inhaled beclomethasone (750 or $1500 \mu \mathrm{g}$ twice daily) in patients with chronic airflow obstruction and PMN function. Twenty patients (seven female) aged 56-77 years were studied (mean (SEM) $\quad \mathrm{FEV}_{1}=1.07 \quad(0 \cdot 10)$ ). Inhaled beclomethasone increased mean (SEM) peak flow rates to $233(19) \mathrm{l} / \mathrm{min}$ from a baseline of 223 (18) and placebo value of 225 (19) (p < $0.05)$. The chemotactic activity of sputum was assessed in eight patients using neutrophils from healthy control subjects. The average number of cells recruited/high power field by the sputum diluted $1: 5$ was 90.8 (SEM 6.5) before treatment and 103.1 (13.4) with placebo, but was lower $72 \cdot 2(6 \cdot 6)$ with inhaled steroids $(p<0.05)$. Spontaneous degranulation of PMN (as measured by fibronectin degradation) was assessed in 10 of the patients. The mean (SEM) baseline values for the patients PMN before treatment was $1.41(0.38) \mu \mathrm{g} / 5 \times 10^{5}$ cells $/ 3$ hours and $1.39(0.19)$ on placebo. There was significant reduction during steroid therapy to 0.73 $(0.17)(p<0.05)$. Finally, the chemotactic response of the patients cells to $10^{-8}$ molar FMLP was reduced during therapy from a baseline value of $67 \cdot 0(6 \cdot 1)$ cells/high power field to $48.1(6.8)(p<0.01)$. The results confirm that inhaled corticosteroids have a small beneficial effect on lung function in patients with chronic airflow obstruction. However, the treatment also reduces the chemotactic activity of sputum and the activation of peripheral PMN. These latter effects may beneficially influence the progression of their lung disease. 
Inflammatory effects caused in the rat lung by inhalation of low levels of ozone

GM BROWN, J SLIGHT, DM BROWN, K DONALDSON Institute of Occupational Medicine, Edinburgh In some cities during the summer the levels of the oxidant gas ozone are sometimes increased. Because of its ability to oxidise, ozone causes injury to the lung but its ability to do so at environmentally plausible levels has been the subject of debate. We examined the ability of ozone, at $0.2-0.8 \mathrm{ppm}$, to cause inflammation, detectable in the bronchoalveolar lavage leucocyte profile, in rats exposed seven hours/day for up to four days. We also assessed the ability of the leucocytes to express proteolytic activity against a fibronectin matrix. Ozone caused a change in the bronchoalveolar leucocyte population only at 0.8 and $0.6 \mathrm{ppm}$ with no effect at 0.4 or $0.2 \mathrm{ppm}$. In addition, the effect was not an increase in total leucocyte numbers but was a change in the percentage neutrophils (data given as mean (SEM) percentage neutrophils): $\mathbf{0 . 8} \mathrm{ppm}$ ozone-control $0.87(0.25), 1$ day $8.53(2.34), 2$ days 4.00 $(0.58), 4$ days $0.32(0.01) ; 0.6 \mathrm{ppm}$ ozonecontrol $0(0), 1$ day $1.99(0.50), 2$ days 3.11 $(0.74), 4$ days $0.11(0.62)$ (significant increase ( $p<0.05$ ) on days 1 and 2). Despite the evidence of low level inflammation, the bronchoalveolar leucocytes did not show increased ability to degrade fibronectin: ${ }^{125} \mathrm{I}$ fibronectin breakdown (mean (SEM)) cpm1 day $0.8 \mathrm{ppm} 6830$ (1086), control 6418 (950); 2 days 0.8 ppm 6042 (999), contro 6165 (928); 4 days 0.8 ppm 4119 (897) control 4055 (829) (NSD). As judged by bronchoalveolar lavage profile, ozone causes detectable inflammation only at 0.6 and 0.8 ppm and an adaptation response is evident, the response decreasing at four days. At these higher levels leucocyte protease mediated injury does not appear to be a likely mechanism in lung injury caused by ozone.

Research funded by the Health Effects Institute.

Lymphoid cell stimulation and interleukin-1 like activity by neutrophils from quartz exposed rat lung

K DONALDSON, $Y$ KUSAKA, RT CULLEN Institute of Occupational Medicine, Edinburgh, and University of Osaka, Osaka, Japan In pneumoconiosis the link between pulmonary deposition of pathogenic dust and immunomodulation, which has been described in several studies, is not at all clear. We therefore examined the effect of inflammatory bronchoalveolar leucocytes (BAL) from quartz exposed rat lung, on lymphocytes in culture. The BAL were added, at varying ratio, to syngeneic $T$ lymphocytes responding to suboptimal PHA. Whole quartz BAL inhibited splenic lymphocyte responses to much the same extent as control BAL (all data as means (SD): ${ }^{3} \mathrm{H}$ thymidine uptake (cpm) in response to mitogen with BAL at 8:1 effector:target ratio: splenocytes alone 7542 (1309), control BAL (97\% macrophages) 346 (43), quartz BAL (50\% macrophages, $50 \%$ neutrophils) 292 (93). On separation of the macrophages and neutrophils into enriched populations we noted that the quartz macrophages were only slightly less inhibitory to $T$ lymphocytes but the neutrophils caused enhanced responses: $T$ lymphocyte response as above with $\mathrm{BAL}$ at 1:8: lymphocytes alone 7714 (756), + quartz macrophages $640(344)(\mathrm{p}<0.001)$, + quartz neutrophils 20518 (1142) (p $<0.001)$. The same quartz neutrophils produced interleukin-1 (IL-1) activity in the thymocyte bioassay: IL-1 level as assessed by enhancement of thymocyte mitogenesis (means (SD) $\mathrm{cpm}$-Control (thymocytes alone) 578 (74), quartz macrophages $1067(116)(\mathrm{p}<0.001)$ quartz neutrophils 1193 (134) ( $p<0.001)$. A role for neutrophil immunostimulation and IL-1 should be considered in situations where neutrophils are recruited to the lung parenchyma.

Research funded by the European Coal and Steel Community.

Routine microbial investigation in community acquired pneumonia: does it help patient management?

J ARROWSMITH, MA WOODHEAD, R CHAMBERLAIN-WEBBER, S WOODING, IP WILLIAMS St George's Hospital, London, and St Albans City Hospital, St Albans The aim of the study was to determine how patients with community acquired pneumonia (CAP) are routinely investigated and whether the results obtained influence management. One hundred and six (78 at St George's, 28 at St Albans) adult (age 21-94) patients with CAP were studied prospectively. Patients were identified at the time of admission and the case notes were then reviewed three or more months later. No attempt was made to influence patient investigation or management. Patient groups and investigations performed were similar in the two hospitals. The following investigations were performed: blood cultures in $86(81 \%)-9(11 \%)$ positive, sputum in $48(45 \%)-19(40 \%)$ positive and paired serology in $14(13 \%)-2$ $(14 \%)$ positive. Microbial aetiology was determined in $29(26 \%)$ and $16(15 \%)$ died. Pathogens included Streptococcus pneumoniae (18), Haemophilus influenzae (8), and Legionella pneumophila (1). Initial antibiotics were prescribed blind and were subsequently altered in $33(31 \%)$ cases, in $13 / 29(45 \%)$ microbiologically positive and $20 / 77(26 \%)$ of those negative $(p>0.05)$. Reasons for change in antibiotics included: clinical changes 18 $(55 \%)$, microbial results $9(27 \%)$, and not known $6(18 \%)$-positive microbiological results emerged in only two of these. Routine microbial tests seldom yield clinically useful information in patients with CAP.

Pulmonary disposition of azithromycin following a single $500 \mathrm{mg}$ oral dose

DR BALDWIN, JP ASHBY, JM ANDREWS, R WISE, D HONEYBOURNE Departments of Thoracic Medicine and Medical Microbiology, Dudley Road Hospital, Birmingham The new macrolide antibiotic azithromycin (AZ) has good activity against common Gram positive

\begin{tabular}{|c|c|c|c|c|c|}
\hline \multirow[b]{2}{*}{$\begin{array}{l}\text { Time } \\
\text { (hours) }\end{array}$} & \multicolumn{5}{|c|}{ Mean concentration $(\mathrm{mg} / \mathrm{l})$} \\
\hline & Serum & Sputum & $\begin{array}{l}\text { Bronchial } \\
\text { mucosa } \\
(\text { mg/kg) }\end{array}$ & $E L F$ & $\begin{array}{l}\text { Alveolar } \\
\text { macrophage } \\
\text { (mg/kg) }\end{array}$ \\
\hline $\begin{array}{l}12 \\
24 \\
48 \\
72 \\
96\end{array}$ & $\begin{array}{l}0.17 \\
0.03 \\
0.03 \\
0.02 \\
-\end{array}$ & $\begin{array}{l}0 \cdot 28 \\
0 \cdot 41 \\
2 \cdot 00 \\
0 \cdot 64 \\
0 \cdot 29\end{array}$ & $\begin{array}{l}1.45 \\
3.50 \\
4.74 \\
3.02 \\
2.77\end{array}$ & $\begin{array}{l}1.01 \\
1.53 \\
1.56 \\
0.48 \\
0.67\end{array}$ & $\begin{array}{c}1.97 \\
6.32 \\
26.59 \\
8.43^{\star} \\
15.55\end{array}$ \\
\hline
\end{tabular}

$\star$ One patient only. and negative bacteria including Legionella pneumophila. In this study we measured serum, bronchoscope aspirated sputum, bronchial mucosal, alveolar macrophage (AM) and epithelial lining fluid (ELF) concentrations of $A Z$ in 16 patients undergoing fibreoptic bronchoscopy for diagnostic purposes. Patients received $\mathrm{AZ}$ as a single $500 \mathrm{mg}$ oral dose between 12 and 96 hours prior to sampling serum, sputum, bronchial mucosa and $200 \mathrm{ml}$ bronchoalveolar lavage fluid (BALF). BALF was centrifuged and the supernatant removed immediately and freeze dried to yield a 20 fold concentration. A tota cell and differential count were performed and the cells were then ultrasonicated following addition of phosphate buffer $\mathrm{pH} 8$. All specimens were assayed using a microbiological plate assay. ELF volume was calculated using total protein concentration of the BALF as a reference. The results are tabulated. AZ achieves high tissue concentrations and even higher AM concentrations well above the $\mathrm{MIC}_{90}$ for common respiratory hours but persisted in bactericidal concentrations beyond 96 hours. These data suggest that $\mathrm{AZ}$ may be useful in the treatment of chest infections and in particular those involving intracellular pathogens.

\section{Psittacosis in pigeon fanciers}

SJ BOURKE, CE FREW, D CARRINGTON, G BOYD Departments of Respiratory Medicine Psittacosis may cause a severe pneumonia (Bourke. J Infect 1989;19:41), and its incidence in the UK seems to be rising. Recently, a new strain, designated Chlamydia pneumoniae TWAR (Taiwan acute respiratory), has been identified and initial reports suggest that-unlike $C$ psittaci-it does not have an avian host (Grayston. Chest 1989;95:664). Many studies have relied upon a complement fixation test (CFT) for diagnosing psittacosis but this test detects antibodies to any member of the genus Chlamydia. We report the results of a detailed study of chlamydial antibody responses in 271 pigeon fanciers, who donated a blood sample and gave details of any previous pneumonia, smoking status, and intensity and duration of avian exposure. Type specific microimmunofluorescent (MIF) tests, incorporating a yolk sac antigen control to exclude false positive cross reactions, showed that 161 $(59 \%)$ fanciers had IgG antibodies to $C$ psittaci; $226(83 \%)$ to TWAR and $33(12 \%)$ to $C$ trachomatis. The CFT result was positive in only $97(60 \%)$ of those with a positive MIF test for $C$ psittaci; while 42 $(28.8 \%)$ sera positive by CFT were negative for $C$ psittaci by the MIF test, indicating the limitations of the CFT in assessing exposure to $C$ psittaci. Only $22(13.6 \%)$ of those with $C$ psittaci and $30(13.2 \%)$ of those with TWAR pathogens. Peak levels appeared in cells at 48 and Microbiology, Royal Infirmary, Glasgow 
antibodies had had pneumonia, suggesting that infection was often mild or subclinical. IgG antibodies to pigeon gammaglobulin were detected in $138(51 \%)$ fanciers but the incidence of TWAR or $C$ psittaci was not related to sensitisation to pigeon derived antigens, number of pigeons kept, time spent in loft, duration of avian exposure, or smoking status. $C$ psittaci infection is common, but usually mild, in pigeon fanciers. The high incidence of TWAR infection at $83 \%$ in this population compared with $19.9 \%$ in general population surveys (Forsey. $J$ Infect $1986 ; 12: 145)$ suggests that this strain might also have an avian reservoir.

High dose inhaled beclomethasone diproprionate in patients with bronchiectasis

JS ELBORN, J CLARKE, B JOHNSTON, F ALLEN, J MCGARRY, DR TAYLOR, G VARGHESE City Hospital, Belfast We have studied the effects of high dose inhaled beclomethasone (B) compared to placebo (P) on symptoms, sputum production and pulmonary function in 20 patients with proved bronchiectasis (mean age 50 years). Eight patients were taking inhaled Salbutamol and two oral theophylline. Beclomethasone $(750 \mu \mathrm{g} / \mathrm{bd})$ or matched placebo was taken double blind for six weeks. Peak expiratory flow rates (PEF) and visual analogue scores (VAS) for cough, wheeze, and shortness of breath were recorded daily in a diary. Sputum was collected daily in sealed containers. Spirometry was measured after three and six weeks. After six weeks patients double blindly crossed over to the alternate therapy and the above measurements were repeated. Mean PEF, $\mathrm{FEV}_{1}$, VAS, and sputum weight for the second 'three week period of each treatment were compared between B and P. Of the 20 patients entered, complete data were available on 15. Three patients were withdrawn owing to infection and two withdrew themselves. $\mathrm{FEV}_{1}$ and $\mathrm{PEF}$ were significantly greater while sputum weight was significantly lower with $B$ than with $P$, as shown in the table, which gives mean (SD) values. A significant improvement in VAS for cough was observed but not for wheeze or SOB. It is concluded that high dose inhaled beclomethasone improves indices of airflow obstruction and reduces sputum volume in patients with bronchiectasis.

\begin{tabular}{|c|c|c|c|}
\hline $\begin{array}{l}F E V, \\
\text { (l) }\end{array}$ & $\begin{array}{l}P E F(a m) \\
(l / s)\end{array}$ & $\begin{array}{l}P E F(p m) \\
(l / s)\end{array}$ & $\begin{array}{l}\text { Sputum } \\
(\mathrm{g})\end{array}$ \\
\hline $\begin{array}{l}\text { B } 2 \cdot 3(1 \cdot 1)^{\star} \\
\text { P } 2 \cdot 2(1 \cdot 1)\end{array}$ & $\begin{array}{l}369(141)^{\star} \\
354(129)\end{array}$ & $\begin{array}{l}383(130) \\
370(116)\end{array}$ & $\begin{array}{l}22(19)^{\star} \\
27(24)\end{array}$ \\
\hline
\end{tabular}

${ }^{\star} \mathrm{p}<0.05 v \mathrm{P}$.

Gentamicin "Rotacaps" achieve therapeutic concentrations in the lung

JM GOLDMAN, SM BAYSTON, $S$ O'CONNOR, RE MEIGH Departments of Respiratory Medicine, Pharmacy, and Microbiology, Castle Hill Hospital, Cottingham Regular administration of nebulised gentamicin and carbenicillin can improve lung function and decrease the frequency of infective exacerbations in patients with cystic fibrosis (Hodson et al. Lancet 1981;ii:1137). Nebulising antibiotics in the home is time consuming, limits mobility, and leads to deposition in the local environment. We investigated a micro- nised gentamicin powder preparation taken via the "Rotahaler" system which is quick clean and convenient to use. We compared the pulmonary deposition of gentamicin achieved by optimal nebulisation to that by the new system. Forty patients attending for routine bronchoscopy were recruited and randomised to inhale gentamicin $160 \mathrm{mg}$ (4 $\mathrm{ml}$ ) from a Turret nebuliser driven at $10 \mathrm{l} / \mathrm{min}$ (group $\mathrm{N}, \mathrm{n}=20$ ) or six capsules each containing $30 \mathrm{mg}$ micronised gentamicin powder via a rotahaler (group $R, n=20$ ). The two groups were similar in age (mean 61 years, range 19-84) and spirometric values (mean (95\% confidence limits) $\mathrm{FEV}, 72 \cdot 1 \%$ predicted (66.0-78.2), FVC $82 \cdot 2 \%$ predicted (76.777.7)). Bronchoalveolar lavage (BAL) of a normal basal segment was performed with $100 \mathrm{ml}$ saline one hour after inhalation. Gentamicin levels were measured on the aspirate and $10 \mathrm{ml}$ of venous blood. There was no significant difference in gentamicin level in BAL fluid between group $\mathrm{N}(\mathbf{9} \cdot 3$ $(13.7-4.9) \mathrm{mg} / \mathrm{l})$ and group $\mathrm{R}(8.0(11.6-4.4)$ $\mathrm{mg} / \mathrm{l})$. The gentamicin level was $<1.0 \mathrm{mg} / 1$ in all serum samples. There was no correlation between time to BAL, volume aspirated, or spirometry, and BAL gentamicin level in the whole population or either group. Gentamicin rotacaps caused cough in some patients, but achieved therapeutic levels in BAL fluid similar to optimal nebulisation of the drug. This novel delivery system for gentamicin may have therapeutic potential.

\section{Long term morbidity from legion-} naires' disease

RS LLOYD, D GUEST, AJ FAIRFAX Department of Thoracic Medicine, Stafford, and Department of Statistics, West Midlands Health Authority In 1985 a major outbreak of legionella pneumonia occurred at Stafford District Hospital. At the time 187 patients were admitted suffering from pneumonia or severe chest infection. Sixty eight had proved Legionella pneumophila infection (22 died) on the basis of exposure to the source, typical clinical features, and positive serological results. We have assessed the morbidity two to three years after recovery in a group of 25 survivors (15 male, 10 female aged 41-85, mean 62.8 years when infected) comparing them with 50 normal controls matched for age, sex and place of residence and with a smaller group of matched patients $(n=12)$ who had suffered pneumonia from othe causes. Assessment comprised a self completed questionnaire, physical examination, and laboratory tests including lung function a quantitative ventilation and perfusion lung scan, and an exercise test. An analysis of variance comparing the three groups indicated persistent disability in the legionella group. The patients were less active and their perceived breathlessness scores and measured walking distances were significantly worse ( $p<0.0001$ ). Spirometry and static lung volumes showed no differences but the TLCo and Kco measurements were significantly lower in the legionella group than in the controls. Minor reductions in ventilation and perfusion were observed on isotope lung scans. These changes were not explained by previous smoking history or prior morbidity. We found no evidence of ong term renal or hepatic damage but there was some evidence of mental impairment in the legionella group from psychometric testing.

\section{A large $Q$ fever outbreak in Birm-} ingham

DL SMITH, JG AYRES, PS BURGE, EG SMITH, MJ wooD Departments of Respiratory Medicine, Microbiology, and Infectious Diseases, East Birmingham Hospital, Birmingham A large urban outbreak of $Q$ fever occurred in April and May 1989 in Birmingham involving 105 identified cases. We present a preliminary report on the clinical findings in 70 cases. Diagnosis was made by a fourfold rise in titre of antibody to phase II antigen of Coxiella burnetti by complement fixation test between acute and convalescent serum samples. A sustained titre of $\geqslant 1: 256$ was also considered diagnostic. Ninety of $105(86 \%)$ were male (mean age 47, range 21-77) and 15 female (mean age 48 , range $8-77$ ); $71 \%$ of patients were admitted to hospital, most of the remainder being seen in hospital clinics. The average length of hospital stay was $7 \cdot 2$ days, range 1-15. In 21 employed individuals an average of 3.8 weeks was lost from work. Some patients remain symptomatic up to four months later. There have been no deaths. The results from 70 questionnaires show fever $(99 \%)$, sweating (93\%) and weight loss $(87 \%)$ to be the most common symptoms. Breathlessness $(67 \%)$ and headache $(66 \%)$, often severe, were frequent; and $63 \%$ had at least one neurological symptom (for example, confusion or speech or vestibular disturbance), in some cases this being the major symptom, a higher frequency than in previous outbreaks. In cases where data are currently available, $93 \%$ had abnormal chest radiographs during their illness, although some had no respiratory symptoms. Twenty five per cent had abnormal liver function test results (mostly elevated transaminases). A case-control study has not indicated a point source of infection; the geographical spread over south east Birmingham suggests airborne spread as the pathway of transmission. Further follow up studies of this, one of the largest $Q$ fever outbreaks reported, are in progress.

Double blind comparison of cefaclor and amoxycillin in the treatment of patients admitted with acute exacerbations of bronchitis and their effect on respiratory tract and oropharyngeal flora

CJ TRIGG, MJ HERDMAN, $M$ WILKS, $S$ TABAQCHALL, RJ DAVIES Departments of Respiratory Medicine and Microbiology, St Bartholomew's Hospital, London Antibiotic treatment is of substantial benefit for patients admitted to hospital with bronchitis, but broad spectrum antibiotics frequently disturb the commensal flora, which provide colonisation resistance, and this may result in susceptibility to respiratory infections. A general practice study (Johanson W et al. Ann Intern Med 1972;77:701) showed a significantly greater prevalence of oropharyngeal colonisation with enterobacteria in amoxycillin treated than in cefaclor treated patients with exacerbations of bronchitis. Cefaclor is increasingly used in such patients because of its efficacy against $H$ influenzae (including $\beta$ lactamase producers) and $S$ pneumoniae without the broad spectrum activity of amoxycillin, which affects the commensal anaerobic flora. We studied 51 patients, mean age 69 (range 46-91) admitted to hospital with exacerbations of severe chronic airflow 
limitation. Twenty six patients received cefaclor ( $250 \mathrm{mg}$ tds for seven days) and 25 amoxycillin (500 $\mathrm{mg}$ tds for seven days) allocated in random, blind fashion. Sputum and throat swabs were collected prior to treatment, on the seventh day, and three weeks after discharge. Infective organisms were isolated in 15 cefaclor (C) and 14 amoxil (A) treated patients. C and A were equally effective at seven days against these organisms but there was a trend towards recurrence in the $\mathbf{A}$ group at follow up. In addition, the $A$ group showed a significantly greater prevalence of abnormal colonisation at follow up $\left(\chi^{2}=4.46, p<0.05\right)$, entirely attributable to the appearance of new organisms ( $C$ albicans, Klebsiella sp and $E$ cloacae.

\section{Airways obstruction: extent of disease by computed tomography and duration of sputum production in bronchiectasis}

BC LEAHY, J WONG, SE CHURCH, PM TAYLOR Departments of Respiratory Medicine and Diagnostic Radiology, Manchester Royal Infirmary, Manchester Diffuse airways obstruction is a common problem for patients with bronchiectasis. The mechanisms responsible for the airways obstruction are not known. This study aims to assess whether the extent of bronchiectasis and the duration of sputum production correlate with the degree of airways obstruction. Twenty five patients with bronchiectasis aged 22 to 75 years (mean 51.8 years) were studied. Each patient was questioned about the duration of their sputum production in years. Forced expiratory volume in one second $\left(\mathrm{FEV}_{1}\right)$ and forced vital capacity (FVC) were measured and compared with predicted values. Computed tomography (CT) of each patient's thorax was performed, the scanning using $10 \mathrm{~mm}$ increments. CT scans were then examined and a score for the extent of bronchiectasis produced according to the number of segments involved and the type of bronchiectasis present. $\mathrm{FEV}_{1}$ was $24-91 \%$ of predicted (mean $48 \%$ ). FVC $45-97 \%$ predicted (mean $68 \%$ ). CT score was 3-26 (mean 13.3). Duration of sputum production was $1-68$ years (mean 29.8). A correlation was found between the severity of airways obstruction $\left(\mathrm{FEV}_{1}\right)$ and both the CT score $(\tau=-0.319)$ and the duration of sputum production $(\tau=$ $-0.364)$. Further analysis showed that $\mathrm{FEV}_{1}$ reduction correlated with duration of symptoms when the effect of the CT score was eliminated $(\tau=-0.30)$ and with CT score when the effect of duration of symptoms was eliminated $(\tau=-0.24)$, suggesting that both the extent of bronchiectasis and the duration of sputum production have an independent effect on the reduction of $\mathrm{FEV}_{1}$.

Influenza vaccination in adults with cystic fibrosis: effect on clinical state and antibody response

ELC ONG, D BILTON, ME ELLIS, EO CAUL, AK WEBB, RA MCCARTNEY Regional Department of Infectious Diseases and Adult Cystic Fibrosis Unit, Monsall Hospital, Manchester, and PHLS Bristol Influenza viruses contribute significantly to the morbidity and mortality of adults with cystic fibrosis (CF). Prevention by immunisation is commonly recommended but there have been no studies assessing the serological response in adults with CF. Twenty nine $C F$ patients ( 18 male, 11 female; mean age 24 years) were immunised with a single dose, intramuscularly, of $0.5 \mathrm{ml}$ of inactivated influenza vaccine (split virion) MFV-JECT (Merieux UK) containing A/ Sichuan/2/76 (H3N2) $15 \mu \mathrm{g} \mathrm{HA}, \mathrm{A} / \mathrm{Sin}-$ gapore/6/86 (H1N1) $15 \mu \mathrm{g} \mathrm{HA}$, and B/Beijing/1/87 $15 \mu \mathrm{g}$ HA during the period October to December 1988. Daily score charts of peak expiratory flow (PEF), temperature, headache, nausea, cough, and pain at site of injection were recorded for 14 days. Antibody response was measured by paired sera at three week intervals using the single radial haemolysis (SRH) test. Eighteen healthy volunteers ( 4 male, 14 female; mean age 44 years) were included. In both $C F$ patients and volunteers there were no significant changes in PEF. Among CF patients two reported fever of $38.5^{\circ} \mathrm{C}$ for the first seven days, five had headaches during the first three days, two had nausea and 15 complained of pain at the site of injection during the first 24 hours Only one patient had increasing cough for the first five days. Significant antibody responses (SRH $>2 \mathrm{~mm}$ were documented in $22 \mathrm{CF}$ patients to $\mathrm{A} / \mathrm{Sich}$ uan/2/87,26 CF patients to B/Beijing $1 / 87$ and $28 \mathrm{CF}$ patients to $A$ Singapore/6/86. Among volunteers, only one had a temperature of $38.5^{\circ} \mathrm{C}$ lasting 24 hours and six had pain at site of injection for three days. Significant antibody responses were documented in 11 volunteers to $\mathrm{A} / \mathrm{Sichuan} / 2$ 87,14 to $B /$ Beijing and 17 to $A /$ Singapore/6 86. In this study the split virion vaccine was well tolerated by CF patients and healthy volunteers and significant antibody responses were documented.

New automated technique for the assessment of emphysema from tissue sections

M GILlOOLY, ASJ FARROW, D LAMB Department of Pathology, University of Edinburgh Quantitative assessment of emphysema requiring manual sampling techniques or using automatic image analysis systems has until now been time consuming. We describe a new automated technique for the assessment of emphysema on histological sections of lung tissue. The fast interval processor (FIP) is a rapid scanning device developed by the staff at the MRC Human Genetics Unit in Edinburgh. The scanning device recognises variations in tissue optical density, and changes in optical density from background to tissue are registered as "hits." Airspace wall surface area per unit volume (AWUV), which we have used to identify loss of surface area and emphysema (Gould et al. Am Rev Respir Dis 1988;137:380), can be derived from the mean linear intercept (LM), where $\mathrm{LM}$ is equal to

\section{$\frac{\text { total test line length }}{\text { number of hits }}$}

The "test line length" is in reality the electronic scan length for each section. AWUV is derived by using the formula

AWUV (surface area) $=\frac{2 \mathrm{~V}}{\mathrm{LM}}$, where $\mathrm{V}=1 \mathrm{~mm}^{3}$ :

$$
\text { that is, } \frac{2}{\mathrm{LM}} \mathrm{mm}^{2} / \mathrm{mm}^{3} \text {. }
$$

The interval between "scan lines" is only 10 $\mu \mathrm{m}$, but the speed of the system is such that sections are scanned at a rate of $2 \mathrm{~mm}^{2}$ per second. The AWUV values obtained with FIP were compared with those measured on the same material with the IBAS automatic image analysis system (Gould et al, 1988). The FIP AWUV results correlated well with the IBAS results $(r=0 \cdot 88)$. The high degree of automation of the process provides very high intraobserver and interobserver reproducibility ( $r=0.996$ and 0.991 respectively) Use of the FIP provides a rapid and accurate technique for the assessment of alveolar surface area and hence microscopic emphysema.

\section{Pulmonary Kaposi's sarcoma}

F MOSS, J FLEMING, J NELSON, D VeALe, B PETERS, D MITCHELL Chest and Allergy Clinic and Wharfside Clinic, St Mary's Hospital (Praed Street), London Pulmonary Kaposi's Sarcoma (KS) is a recognised feature of the acquired immune deficiency syndrome, but its true incidence is not known. Symptoms are usually indistinguishable from respiratory opportunist infections; and transbronchial biopsy is not a good method for identifying parenchymal involvement due to the patchy nature of the disease. However, the distinct lesions of endobronchial $\mathrm{KS}$ may be seen at fibreoptic bronchoscopy and usually do not need biopsy as cutaneous lesions are invariably present. In a series of $122 \mathrm{HIV}$ positive patients with respiratory symptoms who underwent fibreoptic bronchoscopy $30(25 \%)$ had cutaneous KS lesions. In $15(50 \%)$ of these endobronchial KS Lesions were seen. All 15 had ora KS lesions. All had symptoms of cough and dyspnoea. One had haemoptysis. In three patients with extensive endobronchial KS symptoms had been present for more than two months; in one of these no endobronchial lesion was seen at bronchoscopy three months earlier. PCP was found coincidentally in four of the 15 patients $(27 \%)$. The median time from diagnosis of cutaneous KS to the diagnosis of endobronchial $\mathrm{KS}$ was eight months $(0-17)$. Eleven patients have now died. The median survival from diagnosis of cutaneous $\mathrm{KS}$ was 16 months (6-26) and from diagnosis of pulmonary KS four months (1-14). Combination chemotherapy may be associated with both clinical and functional improvement and we have noted regression of mild endobronchial $\mathrm{KS}$ in two patients following chemotherapy. Early diagnosis, before the onset of extensive disease, may result in a favourable response to chemotherapy. The diagnosis of pulmonary $\mathrm{KS}$ should be suspected in HIV positive patients with cutaneous and oral $\mathrm{KS}$ who present with respiratory symptoms.

Tuberculosis and HIV infection in Harare, Zimbabwe

P NEILL, W LEGG, M MAHARI, S RAY, $S$ HOUSTON, L MBENGERANWA City Health Department, Harare, University of Zimbabwe Medical School, Zimbabwe Our object was to examine the clinical picture of tuberculosis in patients with and without HIV infection. Four hundred and forty four patients admitted to the City of Harare Tuberculosis Unit over six months were diagnosed microbiologically (205), clinically (190), or histologically (43). Four hundred and eleven were tested by Elisa for HIV infection. The results are shown in the table.

\begin{tabular}{lcccc}
\hline Age $(y):$ & $<2$ & $3-14$ & $15-40$ & $>40$ \\
\hline Seronegative & 21 & 15 & 142 & 97 \\
Seropositive & 10 & 2 & 105 & 19 \\
\hline
\end{tabular}


In the group 15-40 years the ratio of males to females was 172 to 75 , with a ratio of $75: 30$ in those who were seropositive. Organ involvement: pulmonary 358 (parenchymal 244, hilar nodes 30 , pleural effusion 65 , miliary 19), peripheral lymph nodes 38 , pericardium 15 , meningitis 9 , others $<5$. Significantly more of the following was found in HIV seropositive patients: hilar adenopathy $(\mathrm{p}<0.01)$, peripheral lymphadenitis $(\mathrm{p}<0.001)$, pericarditis $(\mathrm{p}<0.01)$, involvement of two or more organs $(p<$ 0.001 ). No significant difference was found between seropositive and seronegative patients in the following: previous history of tuberculosis, cavitation on chest radiograph, pleural effusion, positive sputum microscopy, Mantoux skin test giving no reaction or $>15 \mathrm{~mm}$ reaction. Numbers of patients with tuberculous meningitis (9), and miliary tuberculosis (19) were small but the trend was for a greater number of seropositive patients with meningitis and no difference for miliary tuberculosis.

\section{Circulating immune complexes and} complement levels in sarcoidosis

DP ROONEY, JS ELBORN, MB FINCH, CF STANFORD Royal Victoria Hospital, Belfast, Northern Ireland Increased levels of circulating immune complexes (CICs) have been described in patients with sarcoidosis but it is unclear if they reflect disease activity. In 31 patients with sarcoidosis CICs (Raji method) and serum complement levels were measured and their relationship to clinical condition examined. Patients were considered to have active disease (group 1) if they presented with bilateral hilar lymphadenopathy and erythema nodosum $(n=5)$ or had worsening of symptoms related to previously diagnosed sarcoidosis $(n=9)$ in addition to either recent deterioration of chest radiographic appearances or reduction in transfer factor for carbon monoxide (TLCO). Patients with no change in symptoms, chest radiography were considered to have stable disease (group 2, $=17$ ). The two groups were well matched for age and sex. Group 1 demonstrated a mean fall in TLCO of $-10 \%$ over the previous 3-6 months whereas it increased in group 2 by $2.7 \%$ ( $p<0.01)$. In group 1 CICs were demonstrated in $11 / 14$ patients, mean 164 (range $60-38) \mu \mathrm{g} / \mathrm{ml}$. In contrast, only $1 / 17$ patients in group 2 had CICs $\left(p<0.001, \chi^{2}\right)$ No abnormalities or differences between groups in serum complement were observed. Serum angiotensin converting enzyme (SACE) was elevated in nine patients from group 1 and four from group 2 ( $p<0.05)$. Clinical improvement was subsequently observed following corticosteroid treatment in nine patients from group 1 with CICs. In all but one of these patients CICs became undetectable 2-6 months later. We conclude that CICs are elevated in patients with sarcoidosis who have clinically active disease, and disappearance of CICs reflects clinical improvement. CICs may be useful in monitoring disease activity in sarcoidosis.

Progressive lung fibrosis 14 years after childhood BCNU chemotherapy: radiological features

PM TAYLOR, BR O'DRISCOLL, HR GATTAMENENI, M PRESCOTT, A WOODCOCK University of Manchester and Wythenshawe Hospital and
Christie Hospital, Manchester We have recently identified a group of eight patients with physiological evidence of pulmonary fibrosis as a late consequence of $\mathrm{BCNU}$ (carmustine) treatment. All eight patients have been studied with thoracic CT, in addition to conventional chest radiography. Scans were performed on an IGE 9800 instrument with $3 \mathrm{~mm}$ sections obtained at $1 \mathrm{~cm}$ increments through the thorax and processed using a high definition "bone" algorithm. Two patients had normal chest radiography and CT scans despite restrictive spirometric defects. The remaining six all showed similar abnormalities but of varying severity. The chest radiographs showed patchy linear upper zone opacities with evidence of volume loss. CT sections demonstrated coarse linear strands lying predominantly in the upper lobes but involving the lower and middle lobes in the two most severely affected patients. The strands lay in the periphery of the lobe and in areas became confluent, producing a high attenuation subpleural band. Although this appeared similar to pleural thickening, one patient was studied in the presence of a spontaneous pneumothorax, and CT scans showed normal parietal pleura and confirmed the sub-pleural band. Seven patients had gallium lung scans; all were normal. These radiological and CT changes are unlike those observed in previously reported cases of drug induced pulmonary fibrosis.

Atypical chest pain in patients with chronic airflow limitation: does 24 hour ambulatory ST segment monitoring facilitate assessment?

R LIM, MJ WALSHAW, $s$ SALTISSI, CRK HIND Department of Medicine, Royal Liverpool Hospital, University of Liverpool It has been suggested that continuous ambulatory STsegment monitoring may help to assess atypical chest pain in patients with chronic airflow limitation (CAL), a group of patients in whom exercise electrocardiographic testing is often unsatisfactory. To investigate this further, we studied 40 such CAL patients (21 males; mean age 61 years, range 24-82). Twenty one patients $(53 \%)$ had ischaemic heart disease (IHD) on the basis of history (typical chest pain) or pathological $Q$ waves on ECG; 13 of these $21(62 \%)$ had stable angina pectoris. We found that $30(75 \%)$ reported recent atypical chest pain which in 21 cases was associated with an acute deterioration of their CAL. All 40 patients then underwent continuous 24 hour ambulatory ST segment monitoring using the Oxford Medilog 4000-II ECG recorder (once in 20 patients, twice in 14 patients, and three times in six patients). Symptom diaries were kept in all 66 recordings. We found 93 episodes $(87 \%$ silent) of significant ST depression (STD) in 13 patients (17 recordings). A greater proportion of these 13 patients had IHD (10/21) than did not (3/19) $\left(\chi^{2}=2 \cdot 15, p<0.02\right)$. Of 148 episodes of chest pain, $131(88 \%)$ were atypical and occurred in 33 recordings. Atypical chest pain correlated with STD in only three recordings (out of 33), whilst angina correlated with STD in four of the nine recordings in which it was reported $\left(\chi^{2}=2.52, p<0.001\right)$. Overall, only 12 episodes of chest pain correlated with STD. The proportion of episodes of atypical chest pain which correlated with STD was markedly lower than that for angina (3/131 $v$ $\left.9 / 17 ; \chi^{2}=7 \cdot 20, \mathrm{p}<0.001\right)$. These observations suggest that 24 hour ambulatory ST segment monitoring may be a useful investigation in elucidating the cause of chest pain in patients with CAL.

\section{Seven years of the British Thoracic} Society: an audit of topics presented

PDO DAvIES South Liverpool Chest Clinic, Liverpool Abstracts of all presentations given at the BTS, which have been published in Thorax, since the creation of the Society in 1982, through to the Winter meeting of 1988 have been analysed by disease studied. The total number of presentations has increased from 59 in summer 1982 to 220 in summer 1988. The commonest disease studied has been asthma, accounting for a relatively constant proportion of all presentations (approximately $25 \%$ ) at most meetings. Certain other diseases, such as chronic bronchitis $(10 \%)$, bronchiectasis/cystic fibrosis $(5 \%)$, lung cancer $(10 \%)$ and tuberculosis $(4 \%)$, have also maintained a reasonably constant proportion of presentations over the seven year period. Other diseases, such as sarcoidosis and pneumonia, have received successively less attention while presentations of animal studies and, not surprisingly, HIV/ AIDS and lung transplant studies have appeared only in recent years. When subjects were analysed further by the experimental technique or topic used in the study of these diseases, studies of treatment were consistently most popular, accounting for between a quarter and a third of all presentations at most meetings. Certain techniques, such as pharmacology (mainly of asthma) and epidemiology (of most diseases), have recently seen an upsurge of interest. Others, such as histology and bacteriology, have received relatively little attention. Though audit of this type may be subject to some interobserver variation, it may provide a useful guide to those seeking worthwhile areas of future research.

Increased plasma concentrations of leucine enkephalin in apical bronchial neoplasms affecting the sympathetic chain

MA GOSNEY, JR GOSNEY, M LYE, CRK HIND Departments of Geriatric Medicine, Pathology, and Medicine, University of Liverpool Leucine (leu-) enkephalin is an opioid peptide present in greatest amounts in the central and autonomic nervous systems, particularly the adrenal medulla. It has also been demonstrated in the normal gut and lung, and in certain neuroendocrine neoplasms including some cases of small cell bronchial carcinoma. Plasma levels of leu-enkephalin can now be measured by radioimmunoassay, which we have therefore performed in 30 patients with histologically proved bronchial carcinoma. We found that plasma levels did not relate to tumour type, or to metastatic disease but did closely relate to the site of the primary growth. Thus in the 23 patients with nonapical neoplasms, plasma leu-enkephalin levels were not significantly different from those found in 10 control subjects without bronchial carcinoma (mean 422, range 218$883 \mathrm{pg} / \mathrm{ml} v$ 426, 322-565). However, in the remaining seven subjects with an apical neoplasm, levels were significantly higher (mean 3050 , range $1259-5820 \mathrm{pg} / \mathrm{ml})(\mathrm{p}<0.001)$. Three of these seven subjects with apical 
disease had one or more components of Horner's syndrome. In no patient was leuenkephalin subsequently demonstrated in their tumour tissue by immunochemistry. These results suggest that elevated levels of leu-enkephalin in subjects with bronchial carcinoma do not reflect neuroendocrine differentiation of the tumour, or tumour metastases in the central nervous system or adrenal glands. Raised concentrations do, however, suggest direct invasion of sympathetic ganglia, from which presumably the peptide is released into the circulation.

This work is supported by the North West Cancer Fund.

Reversal of the adverse effects of nifedipine by aminophylline: a calcium channel facilitator in cor pulmonale

MICHAEL F BONE, LALIT KALRA, ADRIAN PHILLIPS, S ARIARAJ Department of Medicine, Russells Hall Hospital, Dudley, West Midlands Nifedipine, a calcium antagonist with predominant effect on vascular smooth muscle, has been used as a vasodilator in the treatment of heart failure, where it may reduce both preload and afterload. It has also been used to reduce right ventricular strain in cor pulmonale when it may reduce pulmonary artery pressure. Occasionally adverse effects occur with its usage and we describe four cases of cor pulmonale, whom had undergone Swan-Ganz catheterisation and developed adverse haemodynamic effects following the oral administration of nifedipine $20 \mathrm{mg}$. In all four cases intravenous aminophylline, a phosphodiesterase inhibitor which acts as a calcium channel facilitator, was used and immediately corrected these adverse changes with marked improvements in the clinical states. Case 1 developed an acute rise in wedge pressure from 14 to $25 \mathrm{~mm} \mathrm{Hg} 45$ minutes after the oral administration of nifedipine $20 \mathrm{mg}$. This fell to $12 \mathrm{~mm} \mathrm{Hg}$ after aminophylline $250 \mathrm{mg}$ intravenously. $\mathrm{PaO}_{2}$ had fallen from 10.3 to 8.0 and returned to $10.1 \mathrm{kPa}$ similarly and the shunt fraction changed from 15.8 to 34.6 back to $17 \cdot 2 \mathrm{kPa}$ over the same period. In case 2 the adverse effect accompanied marked bradycardia and low output cardiac state, which was immediately corrected by the use of aminophylline. Cases 3 and 4 also developed marked breathlessness following the use of nifedipine and their data showed severe increases in the shunt fraction QS/QT (mean $39.5 \%$ to $53.6 \%$ ) with falls in arterial oxygen saturation $(6.01$ to $5.15 \mathrm{kPa})$ which again responded to intravenous aminophylline (QS/QT $36.7 \%$ and $\mathrm{PaO}_{2}$ 6.4). Calcium antagonists like nifedipine are thought to block the outer voltage dependent gate of the slow channel for calcium. The inner gate, which is functionally different, is phosphorylation dependent and drugs like theophylline which increase cyclic AMP concentration may facilitate calcium flux. This interaction is important to recognise and may be used beneficially or may explain their inefficacy.

Haemodynamic efiects of flosequinan in patients with hypoxic pulmonary hypertension

JS ELBORN, G RICHARDSON, P MURPHY, J MACMACHON City Hospital, Belfast The effects of a new vasodilator, flosequinan (F), on haemodynamics and blood gases was examined in eight patients of mean age 63 years with pulmonary hypertension secondary to hypoxic chronic obstructive lung disease. All patients had a previous hospital admission with heart failure and were stable on diuretic therapy. All had an FEV of $<1.01, \mathrm{PaO}_{2}<8 \mathrm{kPa}$ and a mean pulmonary artery pressure (PAP) $>20 \mathrm{~mm} \mathrm{Hg}$. Patients with a left ventricular ejection fraction $<45 \%$ were excluded. A Swan-Ganz catheter was inserted at least 12 hours prior to the first study day. On day 1, after haemodynamic and blood gas measurements, a matched placebo (P) was given single blind. Measurements were repeated at 4, 8 and 24 hours. The same procedure was repeated the following day after $100 \mathrm{mg}$ of F. All other drug therapy, oxygen administration, daily activity and meal times were kept constant throughout the study days. Significantly lower PAP and pulmonary vascular resistance (PVR) were observed with flosequinan than with placebo (see table).

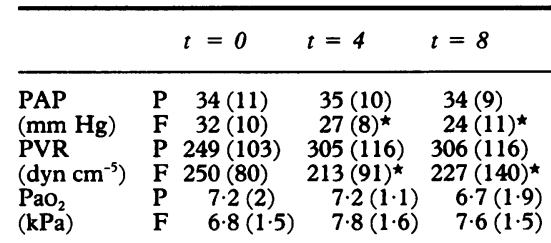

${ }^{\star} \mathrm{p}<0.05 v$ placebo, mean (SD).

A small increase in cardiac output and $\mathrm{SvO}_{2}$ was observed at $\mathrm{t}=4$ and $\mathrm{t}=8$ but differences were not significant. We conclude that flosequinan improved pulmonary haemodynamics without adversely effecting systemic haemodynamics or blood gases in patients with hypoxic pulmonary hypertension. The haemodynamic profile of flosequinan is promising as a pulmonary vasodilator.

\section{Ozone increases bronchial blood flow in} conscious sheep

CE CROSS, ES SCHELEGLE, R GUNTHER, SR COLBERT, GH PARSONS Departments of Medicine and Surgery, University of California School of Medicine, Davis, California, USA The physiological effects of inhaled oxidant gases are generally considered in terms of airway bronchoconstrictive responses. However, it is becoming increasingly evident that air pollutants have effects on other airway functions such as mucus secretion and airway clearance rates. In the present work we have undertaken studies to determine whether ozone $\left(\mathrm{O}_{3}\right)$ could affect bronchial blood flow. Twelve nasally intubated restrained sheep were exposed to $1 \mathrm{ppm}(\mathrm{n}=3), 2 \mathrm{ppm}(\mathrm{n}=3), 3$ $\operatorname{ppm}(n=3)$, or $4 \mathrm{ppm} \mathrm{O}_{3}(n=3)$ for three to four hours. Mean aortic pressure, cardiac output and bronchial artery blood flow (Qbr), measured with a chronically implanted ultrasonic flowmeter (Transonic) or a 20 $\mathrm{MHz}$ pulsed Doppler flow probe, were monitored during $\mathrm{O}_{3}$ exposures. Linear regression analysis revealed a significant correlation $(r=0.94 ; p<0.002)$ between increasing $\mathrm{O}_{3}$ concentration and Qbr. At 3 ppm $\mathrm{O}_{3}$ for three hours the mean increase in Qbr was $77 \%$. Changes in mean aortic pressure and cardiac output were not significantly correlated with increasing $\mathrm{O}_{3}$ concentration ( $\mathrm{r}$ $=0.06$ and 0.47 respectively). Further studies will be required to ascertain the mechanism(s) involved in causing the observed $\mathrm{O}_{3}$ induced airway vasodilation. The results show that $\mathrm{Qbr}$ increases in response to acute $\mathrm{O}_{3}$ inhalation in a dose related manner and that oxidant pollutant induced increases in airway blood flow can be assessed in conscious sheep.

Endothelial cell derived nitric oxide modulates hypoxic pulmonary vasoconstriction

SF LIU, PJ BARNES, TW EVANS Department of Thoracic Medicine, National Heart and Lung Institute, London Decreased production of endothelium derived relaxant factors (EDRF) may play a part in the regulation of hypoxic pulmonary vasoconstriction (HPV). Recent studies have demonstrated that $\mathrm{L}$ arginine is the source of endothelium derived nitric oxide (NO) and that $\mathrm{N}^{\mathrm{G}}$-monomethyl$\mathrm{L}$-arginine (L-NMMA) is a specific inhibitor of NO formation from $\mathrm{L}$-arginine. In the present study we tested the hypothesis that endothelium derived $\mathrm{NO}$ is involved in the regulation of $\mathrm{HPV}$. Lungs from Wistar rats $(300-350 \mathrm{~g})$ were perfused with blood at a constant flow rate and ventilated with gas mixtures of $21 \% \mathrm{O}_{2} / 5 \% \mathrm{CO}_{2} / 74 \% \mathrm{~N}_{2}$ (normoxia), or of $3 \% \quad \mathrm{O}_{2} / 5 \% \quad \mathrm{CO}_{2} / 92 \% \mathrm{~N}_{2}$ (hypoxia). In the first series of experiments, the pulmonary vascular beds were precontracted by adding $15 \mu \mathrm{g}$ prostaglandin $\mathrm{F}_{2 \alpha}$ to the circulation. Acetylcholine (ACh) was then added to the circulation in the presence or absence of $30 \mu \mathrm{M}$ L-NMMA. L-NMMA caused a significant reduction of the endothelium dependent relaxation in response to ACh from 59 (SD 6) \%, 76 (2) \% and 89 (3) \% to $28(6) \%, 48(8) \%$ and $64(5) \%$ at $\mathrm{ACh}$ concentrations of 1,10 and $100 \mu \mathrm{m}$ respectively $(n=4)$, indicating that L-NMMA acts via EDRF. In a second series of experiments two reproducible HPV responses were recorded before and after adding either vehicle, $L-$ NMMA, L-arginine, or L-NMMA plus Larginine. L-NMMA caused a dose dependent augmentation in HPV of $20(5) \%, 32(8) \%, 34$ (8) \% and $77(23) \%$ at concentrations of 30 $300,1,000$ and $2,000 \mu \mathrm{M}$ respectively ( $p<0.05$ compared with control group at all concentrations, $\mathrm{n}=3-5$ ). L-arginine $6 \mathrm{mM}$ reduced HPV significantly (from -11 (4) $\%$ in the control group to $-47(12) \%$ in the $L$ arginine group; $p<0.05, n=5)$. The augmentation of HPV by $30 \mu \mathrm{M}$ L-NMMA was completely reversed by $1 \mathrm{mM}$ L-arginine (\% augmentation, $-11(4) \%,+20(5) \%$ and -12 (3) $\%$ for control, L-NMMA and LNMMA plus L-arginine group respectively; $p<0.05, n=5$ ). We conclude that (1) LNMMA inhibits endothelium dependent relaxation to $\mathrm{ACh}$ and potentiates $\mathrm{HPV}$ in the rat; (2) L-arginine inhibits HPV and reverses the augmentation induced by L-NMMA on HPV; (3) endothelium derived $\mathrm{NO}$ is involved in the regulation of HPV.

Histamine causes dose dependent relaxation and contraction of human bronchial arteries in vitro

SF LIU, DG MCCORMACK, TW EVANS, PJ BARNES Department of Thoracic Medicine, National Heart and Lung Institute, London The role of bronchial vessels in asthma is receiving increasing attention but little is known of the factors that regulate bronchial 
blood flow in man. We have therefore studied the effects of histamine on human bronchial arteries in vitro and analysed the histamine receptors present. Human bronchial arteries (100-200 $\mu \mathrm{M}$ id) were obtained from patients undergoing heart-lung transplantation. The vessels were cut into rings $3 \mathrm{~mm}$ in length and mounted over a pair of fine rigid wires connected to a force transducer and suspended in an organ bath containing KrebsHenseleit solution $\left(37^{\circ} \mathrm{C}\right)$. To study relaxation, the vessel rings were precontracted with prostaglandin $\mathrm{F}_{2 \alpha}\left(10^{-6} \mathrm{M}\right)$ and cumulative dose-response curves for histamine $\left(10^{-2}-10^{-3} \mathrm{M}\right)$ obtained. To study contraction, histamine was added to the organ bath in a cumulative manner when the vessels were at resting tone. In some experiments, the bronchial artery rings were preincubated with $10^{-6} \mathrm{M}$ mepyramine $\left(\mathrm{H}_{1}\right.$ antagonist $)$ or $10^{-4} \mathrm{M}$ cimetidine $\left(\mathrm{H}_{2}\right.$ antagonist) before studying relaxation or contraction. In precontracted vessels, histamine caused dose dependent relaxation at low concentrations $\left(10^{-12}-10^{-6}\right.$ $M)$ and contraction at high concentrations $\left(10^{-4}-10^{-3} \mathrm{M}\right)$. The relaxant response was significantly antagonised by mepyramine with a $\mathrm{pK}_{\mathrm{B}}$ value of 8.3 and the contractile response was also significantly antagonised by mepyramine. In the vessels at resting tone, histamine had no effect at low concentrations $\left(10^{-12}-10^{-6} \mathrm{M}\right)$ and induced dose dependent contraction at high concentrations $\left(10^{-4}-10^{-}\right.$ ${ }^{3} \mathrm{M}$ ). This contraction was virtually abolished after pretreating the vessels with mepyramine. Preincubating the vessels with cimetidine had no significant effect on either the relaxant $\left(-\log \mathrm{IC}_{50} 7.46(\mathrm{SEM} 0.65) v\right.$ $6.23(0.47))$ or the contractile responses. Our data demonstrates that histamine induces dose dependent effects on human bronchial arteries, causing relaxation at low concentrations, and contraction at high concentrations. Both the relaxant and contractile responses are mediated via $H_{1}$ receptors.

L-NMMA reverses the relaxation induced by acetylcholine in rat pulmonary artery

DE CRAWLEY, TW EVANS, PJ BARNES National Heart and Lung Institute, London Evidence increasingly suggests that many vasodilators, such as acetylcholine ( $\mathrm{ACh}$ ), act by releasing a relaxant factor from endothelial cells. Endothelium derived relaxing factor (EDRF) has now been identified as nitric oxide (NO) and is generated via an unknown pathway from the terminal guanido nitrogen atom of the amino acid L-arginine. Recent studies have shown that the $L$-arginine analogue NGmonomethyl-L-arginine (L-NMMA) is an inhibitor of the production of NO from Larginine. In order to study the role of endogenous $\mathrm{NO}$ in the control of pulmonary vessels, we have investigated the effects of $L$ NMMA and L-arginine in the rat pulmonary artery. The right and left pulmonary arteries from male Wistar rats (300-350 g) were dissected free of surrounding tissue and cut into rings $3 \mathrm{~mm}$ in diameter. These were mounted on two parallel wires and pretensed to $500 \mathrm{mg}$, suspended in oxygenated Krebs solution. Relaxation was studied by preconstricting the tissue with $1 \mu \mathrm{M}$ phenylephrine and constructing a cumulative dose-response curve to ACh. L-NMMA, L-arginine and Darginine were added to the baths 10 minutes prior to adding ACh. L-NMMA caused a dose-dependent reversal of the relaxation induced by $\mathrm{ACh}$ at concentrations above $30 \mu \mathrm{M}(\mathrm{p}<0.05)$. Pretreatment with Larginine reversed the inhibition of the ACh induced relaxation caused by $100 \mu \mathrm{M}$ LNMMA ( $<<0.05$ at doses from $10 \mu \mathrm{M}$ to $10 \mathrm{mM} \mathrm{L}$-arginine). Ths isomer of L-arginine, D-arginine $(100 \mu \mathrm{M})$ was ineffective in this respect. We have shown that L-NMMA inhibits endothelium dependent relaxation to $\mathrm{ACh}$ in the rat pulmonary artery, an effect that can be blocked by L-arginine. This suggests that the production of $\mathrm{NO}$ generated in pulmonary vascular tissue can be blocked by L-NMMA, which may help elucidate the role of EDRF in pulmonary vascular control in both health and disease.

Work supported by the Chest, Heart, and Stroke Association

Effect of treatment with high dose inhaled corticosteroids on bronchoalveolar lavage (BAL) fluid infiammatory cells in asthma

M DUDDRIDGE, C WARD, DJ HENDRICK, EH WALTERS Chest Unit, Newcastle General Hospital, University of Newcastle upon Tyne Airway inflammation is thought to be important in the pathophysiology of asthma with increased inflammatory cell numbers and activity in BAL fluid. The effect of inhaled steroids, efficacious as asthma therapy, on airway inflammation is poorly understood. We have assessed the effects of treatment with inhaled beclomethasone $2000 \mu \mathrm{g}$ daily on the findings in serial BAL in 20 volunteer asthmatic patients clinically requiring increased therapy: age $20-52$ years, three regular smokers, 16 initially on only salbutamol $200 \mu \mathrm{g}$ as needed and four on beclomethasone $<200 \mu \mathrm{g}$ daily in addition. Airways responsiveness $\left(\mathrm{PD}_{20} \mathrm{FEV}_{1}\right.$ methacholine) was measured 1-5 days before a $3 \times$ $60 \mathrm{ml} \mathrm{BAL}$ was performed in a segment of the middle lobe. Total and differential cell counts were made and pulmonary macrophage (AM) and granulocyte (Gran) reactive oxygen species generation assessed by lucigenin and luminol amplified unstimulated and latex stimulated chemiluminescence respectively, geometric means expressed as cps $/ 10^{3}$ cells Serial studies were performed a median 2.5 months (range 2-10 months) after steroid therapy was commenced. All patients showed a symptomatic improvement, mean FEV increased by $7 \%$ (SEM $4 \%$ ) of predicted, $\mathrm{p}<0.05$, and geometric mean $\mathrm{PD}_{20} \mathrm{FEV}$ improved from 132 to $254 \mu \mathrm{g}, \mathrm{p}<0.05$ Geometric mean cell counts ( $\times 10^{3} / \mathrm{ml}$ BAL $)$ before and after therapy shown in the table. In contrast to these cellular changes, there were no significant changes found in either unstimulated or stimulated AM or granulocyte activity after therapy. No simple relationship was found between the changes in cell profile or cell activity and improvement in airways responsiveness.
Response to oral corticosteroid treatment in sarcoidosis as assessed by serial bronchoalveolar lavage (BAL)

M DUDDRIDGE, C WARD, DJ HENDRICK, EH WALTERS Chest Unit, Newcastle General Hospital, University of Newcastle upon Tyne Lymphocytosis and increased reactive oxygen species (ROS) release from pulmonary macrophages (AM) and neutrophils (PMN) are found in BAL in sarcoidosis (Thorax 1988;43:787). We have investigated the BAL cell profile and release of ROS by both unstimulated and maximally latex stimulated AM and granulocytes before and immediately after 4-5 weeks' treatment with oral prednisolone $40 \mathrm{mg}$ daily by serial BAL in 12 patients with histologically proved sarcoidosis. They were heterogeneous with respect to their radiological grade, 1-3, and baseline pulmonary function, mean FVC $92 \%$ (SEM 5\%) and TLCo $71 \%(5 \%)$ of predicted. A $3 \times 60 \mathrm{ml}$ BAL was performed in a segment of the middle lobe and total and differential cell counts were made. Luminol and lucigenin amplified chemiluminescence (CL) were measured as indices of ROS generation by granulocytes and AM respectively, and results expressed as cps $/ 10^{3}$ cells. Geometric means before and after treatment were compared by paired $t$ test for 10 patients in whom sufficient cells were harvested on both occasions. There were significant increases in $\mathrm{FEV}_{1}(4 \%(2 \%))$ and FVC $(5 \%$ $(2 \%), \mathrm{p}<0.05)$ and in TrCo $(11 \%(2 \%), \mathrm{p}<$ $0.0001)$. The total cell count did not significantly change, although the absolute lymphocyte count significantly fell from 75 to 46 $\times 10^{3} / \mathrm{ml}\left({ }^{\star}\right)$, the eosinophil count from 1.0 to $0.4 \times 10^{3} / \mathrm{ml}\left({ }^{\star}\right)$ and the epithelial cell count from 2.2 to $0.9 \times 10^{3} / \mathrm{ml}\left(^{\star}\right)$ after steroid therapy $\left({ }^{\star} p<0.05,{ }^{\star \star} p<0.01\right)$.

\begin{tabular}{llllll}
\hline & \multicolumn{2}{l}{$\begin{array}{l}\text { Granulocytes } \\
\text { activity }\end{array}$} & & \multicolumn{2}{c}{ AM activity } \\
\cline { 2 - 3 } \cline { 5 - 6 } & Before & After & & Before & After \\
\hline Unstimulated & 196 & $68^{\star}$ & & $19 \cdot 1$ & $8 \cdot 3^{\star \star}$ \\
Stimulated & 3610 & $1950^{\star \star}$ & 447 & $321 \mathrm{NS}$
\end{tabular}

Thus the effects of steroids in sarcoidosis are complex, involving changes in both cell number and cellular metabolic activity. Further evaluation of the total inflammatory insult to the lung (cell number $x$ cell activity) is required in assessment of the disease and its response to treatment.

Expression of markers of cell activation by bronchoalveolar lavage (BAL) cells from heart-lung transplants (HLT)

JA MONK, TW HIGENBOTTAM, CA CLELLAND, JP SCOTT, J WALLWORK Heart-Lung Transplant Research Unit, Papworth Hospital, Cambridge BAL lymphocyte counts and specifically counts of $\mathrm{T}$ cells expressing CD8 positivity may be increased during acute lung rejection (AR) (Clelland et al. BTS proceedings. Thorax 1989;44:873P). There may also be small increases in total BAL lymphocyte

\begin{tabular}{|c|c|c|c|c|c|c|c|}
\hline & Total & $A M$ & Lymph & $P M N$ & Eos & $E p i$ & Mast \\
\hline $\begin{array}{l}\text { Before } \\
\text { After }\end{array}$ & $\begin{array}{l}210 \\
185\end{array}$ & $\begin{array}{l}183 \\
85 \cdot 5 \% \\
154 \\
81 \cdot 3 \%\end{array}$ & $\begin{array}{l}17 \\
9 \cdot 7 \% \\
22 \\
12 \cdot 5 \%\end{array}$ & $\begin{array}{l}1.8 \\
0.9 \% \\
2.3 \\
1.5 \%\end{array}$ & $\begin{array}{l}1 \cdot 1 \\
0 \cdot 6 \% \\
0.5 \star \\
0 \cdot 3 \%\end{array}$ & $\begin{array}{l}3.5 \\
1.9 \% \\
2.1 \star \\
1.4 \%\end{array}$ & $\begin{array}{l}0.13 \\
0.08 \% \\
0.07 \star \\
0.05 \%\end{array}$ \\
\hline
\end{tabular}

${ }^{\star} \mathrm{p}<0.05$ for an increased lymphocyte or decreased other count. 
counts during pulmonary infection. In an attempt to further differentiate lung infection from AR we have examined 50 cytospin preparations from $30 \mathrm{HLT}$ recipients without knowledge of the clinical diagnosis. The cytospin preparations were stained for so called markers of cell activation, HLA-DR and interleukin-2 (IL-2) using an APAAP technique. Each lavage episode was allocated a category: well (W), AR, treated rejection (TR), infection (I) or chronic rejection (CR) on the basis of the following: clinical state, culture results from sputum and BAL and the histological appearances of transbronchial lung biopsy specimens (TBB). The amount of positive staining was assessed semiquantitatively.

\begin{tabular}{|c|c|c|c|c|c|c|}
\hline & \multicolumn{3}{|c|}{$H L A-D R$} & \multicolumn{3}{|c|}{$I L-2$} \\
\hline & - & + & ++ & - & + & ++ \\
\hline $\begin{array}{ll}\text { W } & (\mathrm{n}=7) \\
\text { AR } & (\mathrm{n}=15) \\
\text { TR } & (\mathrm{n}=6) \\
\mathrm{I} & (\mathrm{n}=8) \\
\text { CR } & (\mathrm{n}=13)\end{array}$ & $\begin{array}{l}0 \\
0 \\
0 \\
0 \\
0\end{array}$ & $\begin{array}{l}4 \\
6 \\
3 \\
2 \\
5\end{array}$ & $\begin{array}{l}3 \\
9 \\
3 \\
6 \\
8\end{array}$ & $\begin{array}{r}7 \\
10 \\
4 \\
5 \\
10\end{array}$ & $\begin{array}{l}0 \\
5 \\
2 \\
2 \\
3\end{array}$ & $\begin{array}{l}0 \\
0 \\
0 \\
1 \\
0\end{array}$ \\
\hline
\end{tabular}

All lavage fluid from HLT patients expressed HLA-DR, principally on macrophages but also on other cells. Staining appeared more extensive during $A R$ and $I$ and in patients with CR, although the differences were not marked. The extent of HLA-DR staining of BAL cells in non-transplant controls (Costabel et al. Thorax 1986;41:261) is similar to our experience of HLT patients. By contrast, IL-2 positive cells, all morphologically lymphocytes, were scarce and were seen in the lavage fluid of some patients in each category except the well group. We have previously demonstrated low CD4 cell counts in HLTS, which may account for the paucity of IL-2 staining.

Immunopathological comparison of matched bronchoalveolar lavage and biopsy samples from asymptomatic asthmatics

LW POULTER, C POWER, A NORRIS, B SCHMEKEL, C BURKE Department of Immunology, Royal Free Hospital School of Medicine, London James Connelly Memorial Hospital, Dublin; and Draco Pharmaceutical, Lund, Sweden

Bronchoalveolar lavage is well established as a valuable research technique in the investigation of interstitial lung disease. Its value in investigating asthma has been restricted to studies performed after episodes of bronchospasm where rises in the properties of eosinophils and soluble mediators have been repeated. As the presence of subclinical peribronchial chronic inflammation in asymptomatic asthmatics has been reported previously to this society, the study investigated whether BAL could be of value in identifying and further analysing this phenomena thus avoiding the need for biopsy. Five asymptomatic asthmatic patients underwent bronchoscopy, during which a full BAL $(3 \times 60 \mu \mathrm{l}$ aliquots of saline $)$ was performed on the right middle lobe. Subsequently a right lower lobe bronchial biopsy specimen was removed from the same patient. Cytospins were prepared from the BAL and frozen sections obtained from the biopsy. Both sets of samples were then investigated with immunopathological tests. All biopsy samples showed a mononuclear cell infiltrate in the bronchial lamina propria with a T lymphocyte dominance. The majority of these cells expressed activation markers (CD7, HLA-DR and the "memory cell" associated molecule identified by MoAb UCHL1. All tissues strongly expressed HLA-DR and raised numbers of RFD1 + dendritic cells were present. In contrast, the BAL samples showed a cytological and phenotypic profile not significantly different to normal. It is concluded that BAL does not sample the appropriate lung areas for investigating peribronchial inflammation in asthma.

Bronchoalveolar lavage profile and ${ }^{99}$ Tc DTPA clearance as prognostic factors in asbestos exposed subjects with and without asbestosis

N AL JARAD, AR GELLERT, RM RUDD London Chest Hospital, London We investigated changes with time in breathlessness, lung function and radiological appearances in 30 patients with asbestosis (AS) and 20 asbestos workers without asbestosis (AW). We related the findings to the results of bronchoalveolar lavage (BAL) and epithelial permeability ( T $1 / 2$ LB), measured by half time clearance from lung to blood of an inhaled aerosol of diethylene tetramine pentacetate labelled with technetium ${ }^{99 \mathrm{~m}}$ Tc DTPA), obtained a mean of $4 \cdot 2$ (range $2 \cdot 3-5 \cdot 8$ ) years previously, when we had found that some AS and AW had increased BAL neutrophil percentages and reduced T $1 / 2$ LB. All subjects had undergone $\mathrm{T} 1 / 2 \mathrm{LB}$ and 33 subjects had undergone BAL. There were 12 smokers, 28 ex-smokers and 10 non-smokers. Nineteen healthy smokers (HS) without asbestos exposure were used as a control group for changes in spirometric values. The annual declines in $\mathrm{FEV}_{1}, \mathrm{FVC}$, TLCO and KCO in AS and AW were greater than predicted annual declines, and annual declines in FEV 1 and FVC in AS and AW were greater than in HS (see footnote to table):

\begin{tabular}{lllll}
\hline & $A S$ & $A W$ & $H S$ & $\begin{array}{l}\text { Predicted } \\
\text { decline/y }\end{array}$ \\
\hline $\mathrm{FEV}_{1}(1 / \mathrm{y})$ & 0.14 & 0.11 & 0.05 & 0.02 \\
$\mathrm{FVC}(1 / \mathrm{y})$ & 0.16 & 0.14 & 0.036 & 0.03 \\
$\mathrm{TLCO}_{(\mathrm{mmol} / \mathrm{min} / \mathrm{kPa} / \mathrm{y})}$ & 0.14 & 0.17 & 0.07 \\
$\mathrm{Kco}_{(\mathrm{mmol} / \mathrm{min} / \mathrm{kPa} / \mathrm{y})}$ & 0.06 & 0.08 & & 0.013 \\
\hline
\end{tabular}

Decline in AS and AW compared with HS and with predicted $p<0.002$ in each case; differences between AS and AW were NS in each case.

The annual decline in TLCo correlated with percentage of BAL neutrophils $(r=0.50, p$ $<0.05$ ), and correlated inversely with BAL lymphocytes $(\mathrm{r}=-0.42, \mathrm{p}<0.05)$. In smokers the decline in TLCO correlated inversely with $\mathrm{T} 1 / 2 \mathrm{LB}(\mathrm{r}=-0.50, \mathrm{p}<0.05)$. BAL profiles and T $1 / 2$ LB did not correlate with declines in FEV, and FVC, deterioration of breathlessness or change in radiographic appearance in AS or AW. Annual declines in TLCo and Kco in smokers $(0.34(0.25)$ and $0.12(0.09)$ SI units respectively) were significantly greater than in ex- and nonsmokers combined $(0.14(0.17)$ and 0.06 $(0.06), \mathrm{p}<0.05$ in each case). We conclude that in asbestos exposed subjects with and without asbestosis decline in lung function is accelerated and both BAL and ${ }^{99 m}$ Tc DTPA clearance have predictive value for the decline in lung function.

\section{Inhaled corticosteroids and asthma}

A NORRIS, C POWER, B SCHMEKEL, A CONDEZ, LW POULTER, CM BURKE Department of Respiratory Medicine, James Connolly Memorial Hospital, Dublin, Eire; Department of Immunology, Royal Free Hospital, London; Draco Pharmaceutical Ltd, Sweden We studied five patients with asthma (ATS criteria) on two occasions, a mean eight months apart (range 6-13). After the first visit inhaled budesonide ( $400 \mu \mathrm{g} \mathrm{BD}$ ) was added to their bronchodilator regimen. Computerised spirometry, bronchial hyperreactivity (BHR) to histamine bronchodilator response (DBR); and endobronchial biopsies were performed at each visit for immunopathological investigation of the presence of $\mathrm{T}$ lymphocyte subsets (CD4/CD8), activation markers (CD7, CD25, HLA-DR), and macrophage subsets (RFD1/RFD7) using immunohistological methods on frozen tissue sections. At the second visit spirometry and BDR $\left(\mathrm{FEV}_{1}\right)$ were unchanged, but BHR was reduced almost fourfold ( $\mathrm{PD}_{20} \mathrm{FEV}$ of $7.45 \mathrm{mg} / \mathrm{ml}$ ). Immunopathological analysis of endobronchial biopsies showed persistence of the peribronchial tissue inflammation in all cases but a significant reduction in the involvement of $\mathrm{T}$ lymphocytes. A reduced proportion of the remaining $T$ lymphocytes expressed UCHL1 and a significant reduction in the numbers of RFD1 + macrophages was noted (in two cases no cells of this phenotype were seen). A significant reduction in HLA-DR expression was also observed despite the persistence of inflammatory cells within the lamina propria. We conclude that (1) a cell mediated immune response is present in asthma and (2) this immune response is modified by inhaled steroids.

Reproducibility of acute effects and subsequent changes in bronchial responsiveness following platelet activating factor challenge in normal man

DA SPENCER, SE GREEN, JM EVANS, PJ PIPER, JF COSTEllo Department of Thoracic Medicine, King's College Hospital, and Department of Pharmacology, Royal College of Surgeons, London Platelet activating factor (PAF) causes acute bronchospasm and neutropenia when inhaled by healthy subjects and may increase bronchial responsiveness. $\mathrm{Re}$ producibility of the effects of nebulised PAF $(400-1600 \mu \mathrm{g} / \mathrm{ml} \mathrm{C18PAF})$ was studied in six normal subjects on two separate days, and lyso-PAF was used on a control day. Responsiveness to methacholine $(\mathrm{MCh})$ was recorded before and between six hours and 14 days following PAF or lyso-PAF. Specific airways conductance (sGaw) and flow at $30 \%$ of vital capacity above residual volume $\left(\dot{V} \max _{30}\right)$ were measured and bronchial responsiveness determined as the provocation concentration of $\mathrm{MCh}$ which reduced sGaw by $35 \%\left(\mathrm{PC}_{35} \mathrm{sGaw} \mathrm{MCh}\right)$ and $\dot{\mathrm{V}} \max _{30}$ by $30 \% \quad\left(\mathbf{P C}_{30} \operatorname{Vmax}_{30} \mathbf{M C h}\right)$. Inhaled PAF produced a similar degree of acute bronchospasm and neutropenia on both challenges in five out of six subjects (mean (SD) maximum percentage fall in sGaw: PAF1: $31(23 \cdot 1) \%$; PAF2: 39 (13.1)\%; $\dot{V} \max _{30}$ : PAF1: 28 (26.7) \%; PAF2: 26 (11.9)\%; mean percentage fall in neutrophil count at $5 \mathrm{~min}$ : PAF1: $34(24 \cdot 4) \%$; PAF2: $28(26 \cdot 6) \% ; n=6)$. There was no significant change in either the geometric mean $\mathrm{PC}_{35} \mathrm{sGaw} \mathrm{MCh}$ or $\mathrm{PC}_{30}$ $\dot{\mathrm{V}} \max _{30} \mathrm{MCh}$ for the whole group of in any 
individual subject following PAF. Lyso-PAF had no acute effects and did not alter methacholine responsiveness. This suggests that a single dose of inhaled PAF can provoke a reproducible degree of acute bronchospasm and neutropenia, but does not significantly increase bronchial responsiveness in normal man.

Urinary excretion of thromboxane and prostacyclin metabolites in normal subjects following inhaled thromboxane $\mathbf{B}_{2}$

P WARD, I TAYLOR, $K$ O'SHAUGHNESSY, G TAYLOR, R FULLER, N PRIDE, C DOLLERY Department of Clinical Pharmacology, Royal Postgraduate Medical School, London Increased production of thromboxane (TX) $A_{2}$ may be important in induction of bronchial hyperresponsiveness. We have previously demonstrated that urinary excretion of $\mathrm{TXB}_{2}$ and its metabolites is significantly increased in patients with severe acute asthma but not in atopic volunteers following antigen provocation to the lung (Taylor et al. BTS proceedings. Thorax $1989 ; 44)$. We investigated whether urinary $\mathrm{TXB}_{2}$ and its metabolites reflect $\mathrm{TXA}_{2}$ formation in the lung in a single blind crossover study in eight non-asthmatic male volunteers. Subjects received either $50 \mu \mathrm{g} \mathrm{TXB}$ or placebo vehicle $(5 \%$ ethanol/saline) via a breath activated dosimeter. Inhaled TXB did not affect FEV $_{1}$ at any time. Urine was collected for two specific protective effect towards a wide range of bronchoconstrictor stimuli, we studied the ability of this drug to displace concentrationresponse curves with adenosine 5'-monophosphate (AMP) and methacholine in a randomised, placebo controlled, double blind study of 12 asthmatic subjects. AMP and methacholine were administered as a nebulised solution and diluted in $0.9 \%$ sodium chloride to produce a concentration range of $0.39-400$ and $0.03-64 \mathrm{mg} / \mathrm{ml}$ respectively. Airway response was measured as $\mathrm{FEV}_{1}$. We compared pretreatment with inhaled $F$ ( $c$ $14 \mathrm{mg}$ ) and nebulised placebo matched for tonicity on bronchospasm induced either by AMP or methacholine. Inhaled F administered five minutes prior to challenges reduced significantly ( $p<0.01$ ), the airways sensitivity to AMP ( $\mathrm{PC}_{20} \mathbf{F E V}$ increasing from 30 to $96 \mathrm{mg} / \mathrm{ml}$ ) and that to methacholine $\left(\mathrm{PC}_{20} \mathrm{FEV}_{1}\right.$ increasing from $1 \cdot 1$ to $1.8 \mathrm{mg} / \mathrm{ml}$ ) When expressed as concentration ratios, $F$ produced a protection of the airways of 1.7 and 3.2 fold against bronchoconstriction provoked by methacholine and AMP respectively, the concentration ratio for AMP being significantly greater than those for methacholine $(p<0.05)$. No significant correlation was found between the concentration ratio for each of the agonists and the baseline $\mathrm{PC}_{20}$ values for methacholine and AMP. No side effects were reported throughout the study. We conclude that the present data indicate that inhaled $F$ protects against an indirect bronchoconstrictor stimulus (namely, AMP) and, to a lesser extent, against a direct bronchial agonist (namely, methacholine).

\begin{tabular}{|c|c|c|c|c|c|c|c|c|}
\hline \multirow[b]{2}{*}{$\begin{array}{l}\mathrm{TXB}_{2}(0-4 \mathrm{~h}) \\
\text { Saline }\end{array}$} & \multicolumn{2}{|c|}{$T X B_{2}$} & \multicolumn{2}{|c|}{ 2,3-dinor- $T X B_{2}$} & \multicolumn{2}{|c|}{ 11-dehydro-TXB } & \multicolumn{2}{|c|}{ 6-oxo-PGF $1 \alpha$} \\
\hline & & $\begin{array}{l}(4 \cdot 8-9 \cdot 5) \\
(0 \cdot 8-4 \cdot 2)\end{array}$ & $\begin{array}{l}50 \cdot 9 \\
11.6\end{array}$ & $\begin{array}{r}(36 \cdot 3-71 \cdot 3) \\
(7 \cdot 0-19 \cdot 0)\end{array}$ & $\begin{array}{l}47 \cdot 7 \\
15 \cdot 4\end{array}$ & $\begin{array}{l}(33 \cdot 6-67 \cdot 7) \\
(11 \cdot 1-21 \cdot 4)\end{array}$ & $\begin{array}{l}4 \cdot 7 \\
5 \cdot 2\end{array}$ & $\begin{array}{l}(3 \cdot 3-6 \cdot 2) \\
(2 \cdot 8-9 \cdot 0)\end{array}$ \\
\hline $\begin{array}{l}\operatorname{TXB}_{2}(4-8 h) \\
\text { Saline }\end{array}$ & $\begin{array}{l}1.8 \\
1.5\end{array}$ & $\begin{array}{l}(1 \cdot 2-2 \cdot 6) \\
(0 \cdot 9-2 \cdot 5)\end{array}$ & $\begin{array}{l}12 \cdot 7 \\
15 \cdot 0\end{array}$ & $\begin{array}{l}(9 \cdot 9-16 \cdot 2) \\
(9 \cdot 9-22 \cdot 5)\end{array}$ & $\begin{array}{l}17 \cdot 3 \\
17 \cdot 9\end{array}$ & $\begin{array}{l}(12 \cdot 7-23 \cdot 6) \\
(13 \cdot 7-23 \cdot 2)\end{array}$ & $\begin{array}{l}3 \cdot 3 \\
3 \cdot 3\end{array}$ & $\begin{array}{l}(1 \cdot 8-4.8) \\
(2 \cdot 2-4.5)\end{array}$ \\
\hline
\end{tabular}

consecutive four hour periods. Thromboxane and prostacyclin metabolites were measured by GC-MS (Barrow et al. Biochim Biophys $A c t a$, in press). Urinary prostanoids (ng/ mmol creatinine) are shown as geometric means $(95 \% \mathrm{CI})$ except for 6-oxo-PGF (arithmetic mean). Urinary $\mathrm{TXB}_{2}$ and its metabolites were significantly raised in the first four hours following $\mathrm{TXB}_{2}$ compared with the saline control $(p<0.001)$. This increase was no longer apparent in the second $4 \mathrm{~h}$ collection. 6-Oxo-PGF $\mathrm{P}_{1 x}$, the hydrolysis product of prostacyclin was not affected by inhaled $\mathrm{TXB}_{2}$. We conclude that lung derived $\mathrm{TXA}_{2}$ will appear in urine as unmetabolised $\mathrm{TXB}_{2}$, together with 2,3-dinor-TXB ${ }_{2}$ and 11dehydro- $\mathrm{TXB}_{2}$. This suggests that the high urinary prostanoid levels found in asthmatics may not arise from the lung.

The MRC and ARC provided financial support.

Effect of frusemide inhalation on methacholine and adenosine 5 '-monophosphate induced bronchoconstriction in asthmatic subjects

R Polosa, LCK LAU, ST holgate Medicine 1, Southampton General Hospital, Southampton Some studies have demonstrated that inhaled frusemide $(F)$ has some protective effect towards various bronchoconstrictor stimuli. In order to clarify whether F may have a non-
Differences in receptor stimulated changes in inositol $(1,4,5)$ trisphosphate (INS $\left.(1,4,5) \mathbf{P}_{3}\right)$ mass and $\left[{ }^{3} \mathbf{H}\right]$ INS $(1,4,5) P_{3}$ binding in airway smooth muscle (ASM) and neuronal tissue

ER CHILVERS, RAJ CHALliss, PJ BARNES, SR NAHORSKI Departments of Pharmacology and Therapeutics, University of Leicester; and Thoracic Medicine, National Heart and Lung Institute, London Ins $(1,4,5) \mathbf{P}_{3}$, formed through agonist induced hydrolysis of phosphatidylinositol $(4,5)$ bisphosphate (PtdIns $\left.(4,5) \mathrm{P}_{2}\right)$ binds to intracellular receptors causing release of $\mathrm{Ca}^{2+}$ from internal stores and ASM contraction. We have examined differences in $\left[{ }^{3} \mathrm{H}\right] \operatorname{Ins}(1,4,5) \mathrm{P}_{3}$ binding properties in cerebellar and ASM membranes and compare these differences to varying patterns of agonist-stimulated accumulation of Ins $(1,4,5) \mathrm{P}_{3}$ concentration observed in corresponding tissue slice preparations. Ins $(1,4,5) \mathrm{P}_{3}$ mass were determined using a radioreceptor assay (Challiss et al. Biochem Biophys Res Commun 1988;157:684) in neutralised TCA extracts from bovine trachealis (BT) or rat cerebral cortex slices (CCS) $(350 \times 350 \mu \mathrm{m})$ that had been preincubated in oxygenated Krebs-Henseleit buffer at $37^{\circ} \mathrm{C}$ and stimulated with carbachol (BT 0.1 mM; CC $1 \mathrm{mM}$ ). [ $\left.{ }^{3} \mathrm{H}\right] \operatorname{Ins}(1,4,5) \mathrm{P}_{3}$ binding was examined in " $P_{2}$ " membrane fractions from rat cerebellum and BT homogenates with non-specific binding determined in the presence of $10 \mu \mathrm{M}$ unlabelled DL-Ins $(1,4,5) \mathrm{P}_{3}$. In CCS, carbachol stimu- lated a rapid and sustained increase in Ins $(1,4,5) \mathrm{P}_{3}$ mass (19.8 (SEM 0.9), $28.3(1.4)$ and $30.7(1.9) \mathrm{pmol} / \mathrm{mg}$ protein at 0,10 and $300 \mathrm{~s}$ ), whereas in BT a transient increase was observed (12.9(0.8), $27(1.5), 12.8(1.0) \mathrm{pmol} /$ $\mathrm{mg}$ protein at 0,5 and $30 \mathrm{~s})$ despite continued PtdIns(4,5,) $\mathrm{P}_{2}$ hydrolysis (Takuwa et al. J Biol Chem 1986;261:14670). A marked difference was also observed in the affinities of the membrane binding sites for Ins $(1,4,5) \mathrm{P}$ (cerebellum $\mathrm{K}_{\mathrm{D}} 29.7(4 \cdot 1) \mathrm{nM}$; BT $3.8(0.2)$ $\mathrm{nM}$ ) and in the degree of inhibition of specific $\left[{ }^{3} \mathrm{H}\right] \operatorname{Ins}(1,4,5) \mathrm{P}_{3}$ binding by $\mathrm{CA}^{2+}$ (cerebellum 0, 41, 78\% inhibition; BT 0, 14, $8^{\circ}$ inhibition by $10 \mathrm{nM}, 5 \mu \mathrm{M}$ and $1 \mathrm{mM}$ $\left[\mathrm{Ca}^{2+}\right]$ respectively). These data suggest distinct differences in the way Ins( 1 , $4,5) P_{3}$ induced $\mathrm{Ca}^{2+}$ release is regulated in neuronal and peripheral tissues.

Effect of glucocorticoids in the human monocyte function in vivo and in vitro

G MANSO, AJ BAKER, IK TAYLOR, RW FULLER Department of Clinical Pharmacology, Royal Postgraduate Medical School, London Glucocorticoids, through a presumed antiinflammatory action, are effective treatment of asthma. Dexamethasone inhibits the release of inflammatory mediators in vitro from human macrophages. To determine whether this effect occurs in vivo we have compared the effect of oral prednisolone on the ex vivo generation of thromboxane $\left(\mathrm{TXB}_{2}\right)$ with the effects of dexamethasone on human monocytes in vitro. In vivo study: six normal subjects took $30 \mathrm{mg}$ of prednisolone or placebo for three days before blood was drawn and the monocytes separated by density centrifugation and adherence culture. The cells were then challenged with various concentrations of opsonised zymosan. In vitro study: Monocytes from six drug free subjects were isolated and challenged with opsonised zymosan ( $1 \mathrm{mg} / \mathrm{ml}$ ) after culture with dexamethasone $10^{-9}-10^{-5} \mathrm{M}$ for 16 hours. $\mathrm{TXB}_{2}$ was measured by radioimmunoassay. Results are shown in the table (mean (SEM)).

In vitro

\begin{tabular}{|c|c|c|c|}
\hline \multicolumn{2}{|c|}{ Dexamethasone (M) } & \multicolumn{2}{|c|}{$\begin{array}{l}T X B_{2} \\
\text { (\% inhibition) }\end{array}$} \\
\hline $\begin{array}{l}10^{-9} \\
10^{-8} \\
10^{-7} \\
10^{-6} \\
10^{-5}\end{array}$ & & $\begin{array}{r}104 \\
88 \\
70 \\
57 \\
49\end{array}$ & $\begin{array}{l}(6 \cdot 7) \\
(5 \cdot 4) \\
(6 \cdot 0) \\
(6 \cdot 6) \\
(8 \cdot 1)\end{array}$ \\
\hline \multicolumn{4}{|l|}{ In vivo } \\
\hline $\begin{array}{l}\text { Zymosan } \\
(\mathrm{mg} / \mathrm{ml})\end{array}$ & Placebo & $\begin{array}{l}\text { Pred } \\
(30 n\end{array}$ & $\begin{array}{l}\text { (nisolone } \\
\text { mg/day) }\end{array}$ \\
\hline $\begin{array}{l}0 \cdot 1 \\
0 \cdot 25 \\
0 \cdot 5 \\
0 \cdot 75 \\
1 \cdot 0\end{array}$ & $\begin{array}{ll}42 & (6.4) \\
68 & (8.4) \\
84 & (5.9) \\
94 & (3.4) \\
93 & (3.7)\end{array}$ & $\begin{array}{l}40 \\
63 \\
89 \\
94 \\
75\end{array}$ & $\begin{array}{l}(13 \cdot 6) \\
(26 \cdot 3) \\
(38.9) \\
(35 \cdot 2) \\
(25 \cdot 8)\end{array}$ \\
\hline
\end{tabular}

Dexamethasone in vitro significantly inhibits $\mathrm{TXB}_{2}$ release by $56 \%$ at $10^{-6} \mathrm{M}$; however, prednisolone in vivo had no effect. It has therefore not been possible to confirm an effect of prednisolone on mediator release in vivo. 
Response to inhaled histamine, methacholine, bradykinin, and metabisulphite in smokers with bronchial hyperresponsiveness

CJ DUGGaN, CMS DIXON, PW IND, NB PRIDE Department of Medicine, Royal Postgraduate Medical School, London Most studies of bronchial hyperresponsiveness (BHR) in smokers have examined response to inhaled histamine $(\mathrm{H})$ or methacholine $(\mathrm{Mch})$ which in humans have mainly direct actions on bronchial smooth muscle. Subjects with smoking related airway disease are claimed to be more responsive than asthmatic subjects to anti-cholinergic agents, suggesting a greater role for neurogenic mechanisms in sustaining airway narrowing in smokers. We have compared airway response to directly acting agents ( $\mathrm{H}$ and $\mathrm{Mch}$ ) with the response to inhaled metabisulphite (MBS) and bradykinin (BK), both of which are believed to cause airway narrowing via neurogenic mechanisms, in 10 male smokers with known BHR to $H$ (mean age $61 \cdot 1$ years: range $57-68$ years; mean baseline $\mathrm{FEV}_{1}$ :FVC $71 \%$, range $52-85 \%$ ). Bronchial challenge was by the dosimeter method using doubling concentrations of $\mathrm{H}, \mathrm{Mch}, \mathrm{BK}$ and MBS. Responsiveness was assessed by the dose $\left(\mathrm{PD}_{35}\right)$ resulting in a $35 \%$ fall in specific airway conductance (sGaw) using a computerised constant volume plethysmograph. The four challenges were single blind and performed on two different days, with two hours separating the challenges on each day. Geometric mean $\mathrm{PD}_{3}$ values were $2.57 \mu \mathrm{mol}, 5.86 \mu \mathrm{mol}, 0.81 \mu \mathrm{mol}$ and $85.11 \mu \mathrm{mol}$ for $\mathrm{H}, \mathrm{Mch}, \mathrm{BK}$ and MBS respectively. The slightly higher sensitivity to H compared with Mch ( $\mathrm{p}=0.09$ ) supports previous studies in smokers. Although smokers with BHR to $\mathrm{H}$ respond to doses of MBS and $B K$ which produce no airway narrowing in normal subjects, responsiveness to MBS and $B K$ is probably less intense than in asthmatic subjects. Previous studies in asthmatic subjects in our laboratory have shown mean $\mathrm{PD}_{35} \mathrm{H}: \mathrm{PD}_{35} \mathrm{BK}$ ratios of 14 , and $\mathrm{PD}_{35}$ Mch: $\mathrm{PD}_{35}$ MBS ratios of $0 \cdot 2$, corresponding ratios in these smokers being 3.5 and 0.07 respectively. These studies therefore suggest neurogenic bronchomotor reflexes are relatively less enhanced in smokers than in asthmatic subjects.

Supported by a Royal North Shore Hospital Centenary Fellowship.

Effect of cetirizine on exercise induced bronchoconstriction in patients with asthma

SK GHOSH, C DE VOS, KR PATEL Department of Respiratory Medicine, Western Infirmary, Glasgow G11 6NT and UCB Pharmaceuticals, Belgium The effect of cetirizine, a potent and specific $\mathrm{H}_{1}$ receptor antagonist given orally and by inhalation, was studied in 21 patients with exercise induced asthma (EIA). Twelve patients ( $5 \mathrm{M})$, mean (SEM) age 35.2 (4.3) y, were given either placebo or cetirizine $10 \mathrm{mg}$ bd orally for one week in a double blind randomised order and then exercised on a treadmill for 6-8 min at submaximal workload $2 \mathrm{~h}$ after the final dose. Changes in FEV 1 were recorded and analysed using Student's $t$ and Wilcoxon's tests for significance. There were no significant changes observed in the baseline $\mathrm{FEV}_{1}$ before and after treatment and cetirizine also failed to block EIA. In a further nine patients ( $5 \mathrm{M}$ ), mean age $41.7(3.3) \mathrm{y}$, the effect of $1 \mathrm{ml}$ of nebulised cetirizine $(5 \mathrm{mg} / \mathrm{ml}$ and $10 \mathrm{mg} / \mathrm{ml}$ ) through a Wright nebuliser was compared with a placebo in a double blind trial. Cetirizine significantly modified EIA at both the concentrations. In three patients cetirizine had no effect and in the remaining six responders the inhibitory effect appeared to be dose related; the mean maximum fall $(\%)$ with placebo and cetirizine $5 \mathrm{mg} / \mathrm{ml}$ and $10 \mathrm{mg} / \mathrm{ml}$ were $22.8(4.3), 13.3(1.8)$ and 3.8 (3.9) respectively. Cetirizine $(10 \mathrm{mg} / \mathrm{ml})$ given by inhalation was locally active in the lung as it displaced geometric mean $\mathbf{P C}_{20}$ histamine (Cockcroft's method) by $13 \cdot 1$ fold to the right compared with placebo.

\begin{tabular}{|c|c|c|c|}
\hline & \multicolumn{2}{|c|}{ Baseline $F E V_{l}(l)$} & \multirow{2}{*}{$\begin{array}{l}\text { Maximum } \\
\% \text { fall }\end{array}$} \\
\hline & Before & After & \\
\hline \multirow{3}{*}{$\begin{array}{l}\text { Oral } \\
(n=12) \\
\text { Placebo } \\
\text { Cetirizine } \\
10 \mathrm{mg} \text { bd }\end{array}$} & & & \\
\hline & $3.05(0.22)$ & $3 \cdot 18(0 \cdot 27)$ & $28 \cdot 2(4 \cdot 1)$ \\
\hline & $2.94(0.22)$ & $3 \cdot 16(0 \cdot 21)$ & $\begin{array}{l}27 \cdot 0(3.5) \\
\text { NS }\end{array}$ \\
\hline $\begin{array}{l}\text { Inhaled } \\
(\mathrm{n}=9) \\
\text { Placebo } \\
\text { Cetirizine }\end{array}$ & $2 \cdot 87(0 \cdot 26)$ & $3.00(0.29)$ & $23.4(2.9)$ \\
\hline $5 \mathrm{mg} / \mathrm{ml}$ & $2.99(0.25)$ & $2.99(0.25)$ & $14 \cdot 2(2 \cdot 1)^{\star}$ \\
\hline $10 \mathrm{mg} / \mathrm{ml}$ & $2 \cdot 84(0 \cdot 26)$ & $2.99(0.32)$ & $10 \cdot 2(5 \cdot 1)^{\star \star}$ \\
\hline
\end{tabular}

Sodium metabisulphite induced bronchoconstriction in guinea pigs: role of acetylcholine and tachykinin release

JO LÖTVALL, B-E SKOOGH, RJ LEMEN, PJ BARNES, KF CHUNG Department of Thoracic Medicine, National Heart and Lung Institute, London, UK, and Department of Pulmonary Medicine, Gothenburg University, Gothenburg, Sweden Sodium metabisulfite (MBS), a commonly used perservative, is known to cause bronchoconstriction in asthmatics. We aerosolised MBS or control solution ( $\mathrm{pH} \mathrm{2.7)} \mathrm{to} \mathrm{anaes-}$ thetised guinea pigs pretreated with propranolol ( $1 \mathrm{mg} / \mathrm{kg})$. Lung resistance $(\mathrm{RL})$ was measured after administration of increasing concentrations of MBS in an ultrasonic nebuliser $\left(1 \times 10^{-2}, 2 \times 10^{-2}, 4 \times 10^{-2}\right.$, and $8 \times 10^{-2} \mathrm{M} ; 20$ breaths). Full dose-response provocations were performed for studies of tachyphylaxis. For reproducibility and drug effects we stopped the provocation when RL had increased by $350 \%$, and a second doseresponse challenge was performed in the same animal 15 minutes later, when RL had returned to stable values. $\mathrm{PC}_{350}$ was calculated from all dose-response curves. No animal responded at $1 \times 10^{-2} \mathrm{M}$, but all animals but two responded at $2 \times 10^{-2} \mathrm{M}$ or $4 \times 10^{-2} \mathrm{M}$. There was a high variability in the increase in RL (350-3900\%). Peak RL was reached 67 (SEM 4) seconds after beginning of nebulisation, and recovered to $42(10) \%$ of peak RL one minute later. Peak RL with the control solution was $28(9) \%$. Tachyphylaxis was observed when the highest doses of MBS were given. With partial dose-response challenges, repeated challenges were reproducible. Atropine ( $1 \mathrm{mg} / \mathrm{kg} \mathrm{IV}$ ) had no effect on MBS responses in five animals, compared with eight controls. To determine the role of tachykinins, animals were pretreated with capsaicin $(50 \mathrm{mg} / \mathrm{kg} \mathrm{SC}$ ) and studied one week later. $P_{350}$ in seven capsaicinised animals was not significantly different from that of six controls. Our data show that aerosolised MBS causes bronchoconstriction in guinea pigs, and that this effect is unlikely to be due to release of acetylcholine or tachykinins from airway nerves.

Nocturnal asthma: combined vagal and alpha sympathetic blockade fails to correct completely nocturnal dip

C TEALE, JFJ MORRISON, SB PEARSON Respiratory Unit, Killingbeck Hospital, Leeds Vagal blockade with atropine (At) corrects most of the nocturnal fall in PEF in asthma suggesting that diurnal variation in vagal tone is important in nocturnal asthma although other factors are likely to be involved $(\mathrm{Br} \mathrm{Med}$ $J$ 1988;296:1427-9). We have previously shown that diurnal variation in plasma adrenaline does not contribute (BTS proceedings. Thorax $1989 ; 44: 889 \mathrm{P}$ ); in this study we have given the alpha-blocker phentolamine $(\mathrm{Ph})$ plus At to determine if combined vagal and alpha-sympathetic effects fully explain nocturnal deterioration in asthma. Eight asthmatics with a diurnal variation in PEF of $>20 \%$ were studied. Inhaled bronchodilators were stopped 10 hours before each study. At 4 am we measured baseline PEF. Subjects then received IV $\mathrm{Ph} 30 \mathrm{mg}$ followed by IV At $30 \mathrm{mg} / \mathrm{kg}$ and PEF was measured 10 min later. The procedure was repeated at 4 $\mathrm{pm}$. Results were analysed by paired $t$ tests. Doses of drugs used were determined by previously established dose-response curves. Mean PEF at 4 am was $359 \mathrm{l} / \mathrm{min}$, rising to $4741 / \mathrm{min}$ at $4 \mathrm{pm}(\mathrm{p}<0.01)$. Combined $\mathrm{Ph}$ and At improved PEF at 4 am by $1181 / \mathrm{min}$ (p $<0.001)$ and PEF at $4 \mathrm{pm}$ by $651 / \mathrm{min}(\mathrm{p}<$ 0.01 ). However, combined $\mathrm{Ph}$ and At at nigh improved PEF to only $467 \mathrm{l} / \mathrm{min}$, significantly below the PEF of $5031 / \mathrm{min}$ achieved by $\mathrm{Ph}$ and At during the day $(\mathrm{p}<0.05)$. Our results suggest that $\mathrm{Ph}$ and At producing combined alpha sympathetic and vagal blockade fails to fully correct nocturnal deterioration in asthmatics. The small gap previously demonstrated remains to be explained.

Muscarinic receptor affinity is an important determinant of human bronchial responsiveness to methacholine

MH BOSKABADY, PD SNASHALL Department of Medicine, Charing Cross and Westminster Medical School, London The degree of muscarinic antagonism produced by atropine (A) depend only on its concentration at the receptor and receptor affinity (Arunlakshana and Schild. Br J Pharmacol 1959;14:48). Responsiveness to methacholine $(M)$ is more complex, but concentration and receptor affinity must be determining factors. To determine the importance of these two factors, we have compared responsiveness to $M$ and $A$ in 9 normal and 10 asthmatic subjects. After baseline measurements of specific airways conductance (sGaw) in a body plethysmograph, the subject inhaled stepwise increasing concentrations of $M$ until $\mathbf{s G a w}$ had fallen by over $35 \%$. Sensitivity to $M$ was measured as the cumulative dose required to produce a $35 \%$ fall in $\mathbf{s G a w}\left(\mathrm{PD}_{35}\right)$. On different days over a two week period three measurements of $P_{35}$ were made on each subject. Repeat measurements of $\mathbf{P D}_{35}$ were also made after inhalation of $0.14 \mathrm{mg} \mathrm{A}$ (on two occasions) and intravenous injection of $0.32 \mathrm{mg} \mathrm{A}$. The antagonist effect of $\mathrm{A}$ was measured as (dose 
ratio-1), where $D R=P D_{35}$ after $A /$ baseline $\mathrm{PD}_{35}$. In relating $(\mathrm{DR}-1)$ to $\mathrm{PD}_{35}$ we have used a different estimate of $\mathbf{P D}_{35}$ from that used to calculate (DR-1). In five estimates of the correlation between $\mathrm{PD}_{35}$ and (DR-1) INHALED mean $\mathrm{r}^{2}=0.63(\mathrm{p}<0.002)$; in three estimates of that between $\mathrm{PD}_{35}$ and (DR-1)IV mean $r^{2}=0.45$ ( $\left.<<0.005\right)$. Our results suggest that approximately $60 \%$ of the intersubject variance of methacholine sensitivity is due to variation of factors that control the degree of antagonism produced by inhaled atropine (delivery, receptor affinity) and that $45 \%$ of variance is due to factors that control the antagonist effect of IV atropine (mainly receptor affinity).

Prolonged bronchodilation and protection from methacholine induced bronchoconstriction by the long acting inhaled beta agonist formoterol

GM NICHOL, A NIX, A ROBSON, PJ BARNES, KF CHUNG Department of Thoracic Medicine, National Heart and Lung Institute, London We compared the effect of inhaled formoterol (F, $12 \mu \mathrm{g}$ ) and terbutaline ( $\mathrm{T}, 250 \mu \mathrm{g}$ ) on $\mathrm{FEV}_{1}$ and responses to inhaled methacholine in 12 mild asthmatics (6 M/6 F, mean (SEM) age 25.4 (4) years, $\mathrm{FEV}_{1} 88$ (15) \% predicted) for five hours after inhalation. The study was randomised, placebo controlled, and double blind. Subjects were studied on three separate days over a period of 1-2 weeks after an initial familiarisation visit. FEV 1 and FVC were measured before and again $0.5,1,2,3,4$ and 5 hours after inhalation of the coded medication. Responses to methacholine were determined at 2 and 5 hours by administration of increasing doubling concentrations of inhaled methacholine in $0.9 \%$ saline $(0.125$ $32 \mathrm{mg} / \mathrm{ml}$ ) by the dosimeter method. $\mathrm{PC}_{20} \mathrm{MC}$, the concentration of methacholine causing a $20 \%$ fall in $\mathrm{FEV}_{1}$ from baseline, was calculated by linear interpolation of the log dose-response curve. $\mathrm{FEV}_{1}$ was allowed to recover spontaneously after the 2 hour challenge. F caused significant bronchodilation at 2 hours $\left(F E V_{1}\right.$ rose from $3.34(0.26) 1$ at baseline to $3.68(0.2) 1$ at 2 hours; $p<0.05)$, while neither placebo $(P)$ nor $T$ caused any significant change in $\mathrm{FEV}_{1}$ compared with baseline. There was a significant $2 \cdot 23$ fold increase in $\mathrm{PC}_{20} \mathrm{MC}$ after $\mathrm{F}$ compared with $\mathrm{P}$ at 2 hours ( $p<0.05$ ), while $T$ caused no significant change in $\mathrm{PC}_{20} \mathrm{MC}$ compared with $P$. By 5 hours $F E V_{1}$ after $F$ had recovered to a level similar to that at 2 hours $(3.59(0.2) 1 ; \mathrm{NS}$ compared with 2 hour level). FEV also recovered to the 2 hour level after $P$ and $T$. $P_{20} M C$ after $F$ at 5 hours was 2.92 fold greater than after $P(p<0.05)$, while methacholine reactivity after $T$ at 5 hours was not significantly different from that after $P$. We conclude that as formoterol effectively bronchodilates and protects against induced bronchoconstriction for at least 5 hours it may be clinically useful-for instance, in preventing nocturnal asthma.

Effect of inhaled frusemide on adenosine 5 '-monophosphate and histamine induced bronchoconstriction in asthmatic subjects

BJ O'CONNOR, YM CHEN-WORSDELL, RW FULLER, KF CHUNG, PJ BARNES Department of Thoracic Medicine, National Heart and Lung Institute and Brompton Hospital, London Inhaled frusemide (F) inhibits broncho-

\begin{tabular}{|c|c|c|c|c|}
\hline & \multicolumn{2}{|l|}{$A M P$} & \multicolumn{2}{|l|}{ HIST } \\
\hline & $\log P C_{20}$ & $P C_{20}(\mathrm{mg} / \mathrm{ml})$ & $\log P C_{20}$ & $P C_{20}(\mathrm{mg} / \mathrm{ml})$ \\
\hline $\begin{array}{l}\mathbf{P} \\
\mathbf{F}\end{array}$ & $\begin{array}{l}0.98(0.29) \\
1.59(0.24)^{\star}\end{array}$ & $\begin{array}{r}9 \cdot 5 \\
39 \cdot 1\end{array}$ & $\begin{array}{l}0.09(0.22) \\
0.09(0.16)\end{array}$ & $\begin{array}{l}1.2 \\
1.2\end{array}$ \\
\hline
\end{tabular}

Results are mean (SEM) $\log \mathrm{PC}_{20}$ and geometric mean $\mathrm{PC}_{20}$ with analysis by paired $t$ test. ${ }^{\star} \mathrm{p}<0.01$.

constriction induced by indirect challenge and cough induced by low $\mathrm{Cl}^{-}$solutions. Its mechanism is uncertain. Nebulised adenosine 5'-monophosphate (AMP) produces a bronchoconstriction in atopic and asthmatic subjects which is blocked by $\mathrm{H}_{1}$ receptor antagonists and cromoglycate/nedocromil. In order to further assess the mode of action of $F$ we studied its effects on AMP and histamine challenge in nine asthmatic subjects (table). Each inhaled F, $30 \mathrm{mg}$, or saline placebo (P) - in a randomised double blind manner30 minutes before challenge with doubling concentrations of AMP $(0.04-400 \mathrm{mg} / \mathrm{ml})$ aerosol from a nebuliser attached to a dosimeter. Seven of these subjects also underwent histamine challenge $(0 \cdot 125-32 \mathrm{mg} / \mathrm{ml})$ in similar manner following $F$ and $P . P_{20} A M P$ and $\mathrm{PC}_{20}$ histamine, the concentration of each agent needed to lower $\mathrm{FEV}_{1}$ by $20 \%$, were calculated by linear interpolation of log dose response curves. Pretreatment with $F$ did not affect histamine responsiveness, but caused a significant fourfold reduction in responsiveness to AMP when compared with $P$. Thus $F$ inhibits bronchoconstriction induced by AMP but not by histamine, providing further evidence for an effect independent of airway smooth muscle, which may involve sensory nerves and/or mast cells.

Comparison of rigid and fibreoptic bronchoscopy: the patient's view

SA GOMM, M JONES, AM HILTON, PV BARBER Department of Thoracic Medicine and Surgery, Wythenshawe Hospital, Manchester An assessment of the acceptability and side effects experienced by 100 patients $(72$ male and 28 female) who underwent fibreoptic bronchoscopy (FB) compared with 100 patients (58 male and 42 female) who had rigid bronchoscopy (RB) was obtained by each patient completing a questionnaire one week after the examination. Prior to bronchoscopy, in both groups $94 \%$ received an adequate explanation and there was no significant difference in the degree of anxiety experienced. The commonest symptoms during FB were cough (76\%), dyspnoea (38\%), nasal pain $(37 \%)$, wheeze $(34 \%)$, lightheadedness $(33 \%)$, nausea $(25 \%)$, tremor $(12 \%)$, and chest pain (10\%). Following RB a significant proportion had a sore throat (91\%) compared with 58\% FB ( $<<0.001$ $\chi^{2}=22 \cdot 8$ ). However, after FB a significantly higher incidence of nausea and vomiting occurred, $28 \%$ and $18 \%$ compared with $16 \%$ and $5 \%$ after $\mathbf{R B}\left(\mathrm{p}<0.01, \chi^{2}=8.3\right)$. The incidence of haemoptysis was comparable $28 \%$ and $30 \%$ respectively. Furthermore, the major symptom after $\mathbf{R B}$ was muscular aches and pains $(21 \%)$, which did not occur in the FB group. By choice $38 \%$ of RB patients would not agree to a FB and conversely $23 \%$ of FB patients would not agree to a RB Following bronchoscopy, 75\% FB and $86 \%$ RB would have a repeat examination. In the 19 patients who had an RB prior to $F B, 12$ preferred FB and three had no preference, and in the $27 \mathrm{RB}$ who had had a preceding FB
21 preferred the RB. In conclusion, doctors seriously underestimate the lack of acceptability and side effects experienced by patients whether undergoing fibreoptic or rigid bronchoscopy.

Bronchoscopic local anaesthesia: intratracheal cocaine and lignocaine appear equally effective

C TEALE, PJ GOMES, MF MUERS, SB PEARSON Respiratory Unit, Killingbeck Hospital, Leeds Intratracheal injection of cocaine or lignocaine may provide superior local anaesthesia during bronchoscopy compared with bronchoscopic administration (BTS proceedings. Thorax 1988;43:805P). No studies have compared these two drugs by the intratracheal route. In a double blind study of 60 patients we have compared the effects of intratracheal cocaine and lignocaine. Sixty consecutive patients aged $40-80$ years (mean 66 ) undergoing their first bronchoscopy were studied. All received lignocaine spray to nose and pharynx and IV atropine $0.6 \mathrm{mg}$ but no sedation. They were then randomised to receive cocaine $4 \mathrm{ml}$ $2 \cdot 5^{\circ}{ }_{0}$ or lignocaine $4 \mathrm{ml} 4 \%$ by two intratracheal injections. Local anaesthesia was assessed by cough count, operator acceptability (scale $0=$ very good to $4=$ very poor) and patient discomfort (scale $0=$ none to $3=$ severe). The table shows the mean scores for 30 patients in each group analysed using the unpaired $t$ test. Although the table shows a trend towards better results using cocaine, differences were small and none reached statistical significance. One patient developed transient hypotension following cocaine. We conclude that intratracheal cocaine and lignocaine are equally effective in producing local anaesthesia during bronchoscopy.

\begin{tabular}{llrl}
\hline & Cocaine & Lignocaine & $p$ \\
\hline Coughs & 7.7 & 11.4 & $0.3 \mathrm{NS}$ \\
Operator score & 1.1 & 1.4 & $0.2 \mathrm{NS}$ \\
Patient score & 1.3 & 1.5 & $0.3 \mathrm{NS}$ \\
\hline
\end{tabular}

Variability of inspired oxygen concentration with nasal cannulas

EA BAZUAYE, TN STONE, PA CORRIS, GJ GIBSON Department of Respiratory Medicine, Freeman Hospital, Newcastle upon Tyne Nasal cannulas are often used for delivering supplemental oxygen in acute respiratory failure even though the precise inspired oxygen concentration $\left(\mathrm{FrO}_{2}\right)$ is unknown. We have adapted the expired gas sampling method of Leigh (Anaesthesia 1970;25:210) to estimate $\mathrm{FrO}_{2}$ and its variation within and between seven normal subjects and seven hypoxaemic patients with COPD breathing supplemental oxygen by Venturi mask (" $24.5 \%$ " and " $28 \%$ "), nasal cannulas (1 $\left.1 \mathrm{~min}^{-1}, 21 \mathrm{~min}^{-1}\right)$ 


\begin{tabular}{|c|c|c|c|c|c|c|}
\hline & \multicolumn{2}{|c|}{ Venturi mask } & \multicolumn{2}{|c|}{ Nasal cannulas } & \multicolumn{2}{|c|}{$M C$ mask } \\
\hline & “24.5\%" & “28\%" & $18 \mathrm{~min}^{-1}$ & $2 \operatorname{lmin}{ }^{-2}$ & $2 l \min ^{-1}$ & $4 l \min ^{-1}$ \\
\hline $\begin{array}{l}\text { NORMAL } \\
\text { Mean } \mathrm{FIO}_{2} \% \\
\text { Mean } \mathrm{COV} \% \text { WS } \\
\mathrm{COV} \% \text { BS }\end{array}$ & $\begin{array}{r}25 \cdot 2 \\
0.9 \\
1 \cdot 2\end{array}$ & $\begin{array}{r}30 \cdot 1 \\
0.6 \\
1.7\end{array}$ & $\begin{array}{r}26 \cdot 7 \\
1.9 \\
10 \cdot 1\end{array}$ & $\begin{array}{r}30 \cdot 1 \\
3 \cdot 1 \\
8 \cdot 6\end{array}$ & $\begin{array}{r}37.9 \\
2.0 \\
5.8\end{array}$ & $\begin{array}{r}46.5 \\
3.0 \\
10.5\end{array}$ \\
\hline $\begin{array}{l}\text { Mean } \\
\text { Mean } \mathrm{FIO}_{2} \% \\
\text { MeV \% WS } \\
\text { COV \% BS }\end{array}$ & $\begin{array}{r}25.5 \\
0.9 \\
2.7\end{array}$ & $\begin{array}{r}30 \cdot 3 \\
0 \cdot 9 \\
3 \cdot 0\end{array}$ & $\begin{array}{r}28.9 \\
2.9 \\
14.2\end{array}$ & $\begin{array}{r}29 \cdot 3 \\
3 \cdot 1 \\
13 \cdot 7\end{array}$ & $\begin{array}{r}38.5 \\
3.8 \\
11 \cdot 1\end{array}$ & $\begin{array}{r}47 \cdot 2 \\
4.9 \\
13 \cdot 1\end{array}$ \\
\hline
\end{tabular}

and an uncontrolled oxygen (MC) mask $(2,41$ $\left.\mathrm{min}^{-1}\right)$. Expired air was sampled at the lips by a mass spectrometer and expired oxygen and $\mathrm{CO}_{2}$ concentrations were plotted on an $\mathrm{O}_{2}-$ $\mathrm{CO}_{2}$ diagram, from which $\mathrm{FIO}_{2}$ was extrapolated assuming a respiratory exchange ratio of $\mathbf{0} \cdot \mathbf{8}$. Comparison of the calculated $\mathrm{FIO}_{2}$ with directly sampled inspired air when room air or oxygen was breathed via a Venturi mask confirmed the validity of the method; in addition, in the patients the calculated $\mathrm{Fro}_{2}$ values were consistent with changes in arterial oxygen saturation measured by an ear oximeter. In the table variations in $\mathrm{FIO}_{2}$ are expressed in terms of coefficient of variation (COV) both within (WS) and between (BS) subjects. Although low flow oxygen via nasal cannulas gives similar mean $\mathrm{FrO}_{2}$ to $24.5 \%$ and $28 \%$ Venturi masks, the variation in $\mathrm{FrO}_{2}$ both within and between subjects is considerably greater with cannulas and of similar order to that seen with uncontrolled oxygen delivery.

Oxygen conserving devices: overused in the United States or underused in Britain?

ADRIENNE MARTIN, ANNE BALLINGER, J MOOREGILLON Department of Respiratory Medicine, St Bartholomew's and Homerton Hospitals, London Oxygen conserving devices (OCDs) are designed to co-ordinate oxygen delivery with inspiration, in theory reducing the mean flow rate necessary to relieve hypoxaemia. We surveyed 150 British respiratory physicians, chosen to be representative of all geographical areas and types of practice, to establish the extent of knowledge and use of OCDs. There were 111 respondents, none of whom had any patients currently using an OCD. Sixty nine per cent had heard of OCDs, $43 \%$ recalled seeing publications on them and $35 \%$ described themselves as familiar with the principles of operation of one or more devices. Thirty three per cent recalled seeing them advertised. Thirty seven respondents $(33 \%)$ felt qualified to comment in detail on indications and usefulness. Of these, $23(62 \%)$ thought OCDs could extend the range of portable oxygen equipment and $59 \%$ thought they could reduce the cost of delivering oxygen from fixed installations. Sixty five per cent believed OCDs were underused in Britain and $70 \%$ would like to use them in some patients. Forty six per cent also felt, however, that currently available published data were not sufficiently convincing to merit widespread introduction of OCDs. An estimated 50000 patients in the USA use some form of $O C D$, but so far as we can tell not a single Briton. It may well be the case that some-or even much-American use is inappropriate, but are some British patients and health authorities failing to benefit from genuinely increased convenience and financial savings? The topic deserves wide discussion and further investigation.
Tuberculosis screening of new immigrants

LP ORMEROD Chest Clinic, Blackburn Royal Infirmary, Blackburn The Blackburn DHA was informed of 2033 new immigrants during $1983-8$, but only $55 \%$ were identified by the official port of arrival (POA) system. In all, $1691(83 \%)$ were traced, over $80 \%$ of whom were aged under 30 ; and $29.9 \%$ of those aged under 30 had a negative tuberculin test response on entry and were given BCG vaccination. Of the children under $16,12.8 \%$ required chemoprophylaxis. Only 11 cases of tuberculosis were found on entry. The rate of tuberculin positivity rose from $42 \%$ in the under $5 \mathrm{~s}$ to $91 \%$ in the over $65 \mathrm{~s}$. The problems with the official POA system for reporting new immigrants will be discussed. Entry screening of new immigrants is valuable, allowing preventive action, BCG vaccination, or chemoprophylaxis in a substantial proportion of those screened.

\section{Treatment of pulmonary tuberculosis} in England and Wales

$P$ WILKINSON (on behalf of the British Thoracic Society Research Committee and the Medical Research Council Cardiothoracic Epidemiology Group) Brompton Hospital, London Regimens currently recommended for the treatment of pulmonary tuberculosis (PTB) are: (1) rifampicin (R) and isoniazid $(\mathrm{H})$ for nine months, with ethambutol (E) for the first two months [HRE/HR], (2) isoniazid and rifampicin for six months, with ethambutol and pyrazinamide (Z) for the first two months [HRZE/ $\mathrm{HR}$ ], and (3) isoniazid and rifampicin for six months, with pyrazinamide alone for the first two (HRZ/HR], although this last regimen has never been studied in Britain. To determine the prescribing practices in England and Wales, the treatments of all 1136 identified adult cases of PTB notified in the first six months of 1988 were surveyed. The preliminary data, which are available for 617 culture proved cases, show that many patients failed to complete therapy as planned: of the 454 patients who were known to have completed therapy at the time of the survey, 82 $(18 \%)$ had not completed as intended (information not available on a further 13 cases). Drug toxicities occurred with similar frequency in each regimen $14 \%$ of those starting on HRZE, $13 \%$ of those starting on HRZ and $9 \%$ of those starting on HRE). There was wide variation in the durations of both the initial and continuation phases of chemotherapy: only $90(46 \%)$ of the patients on HRZE/HR and $50(25 \%)$ of the patients on $H R Z / H R$ received therapy approximating to suggested schedules (initial phase of 6-12 weeks, total duration of 5-7 months), with $51 \%$ of patients receiving therapy for more than seven months. Despite introduction of pyrazinamide into therapeutic regimens, there has not been a general move towards shortening these treatments to six months.
Does a positive Heaf test response predict later breakdown of pulmonary tuberculosis in Asian schoolchildren?

JB COOKSON, AGI cookson Glenfield General Hospital, Leicester, and Central Community Unit, Leicester The Department of Health recommends that those children with a positive tuberculin test response when tested in schools at the age of 12 or 13 should receive chemoprophylaxis against tuberculosis. In Leicester, with a large Asian population, this poses major resource problems. We have therefore investigated the likely "breakdown" of such patients to assess the potential benefits of such a policy. Total tuberculosis notifications in Leicester in all ages varied from 159 to 241 per year for the years 1983-7. Notification forms for the years 1983-7 were scanned for subjects with Asian sounding names who would have been aged around 12 and 13 in 1982 and 1983 . These names were then compared with the lists of those children who had had grade 3 or 4 Heaf test responses in 1982 and 1983. Birth dates and addresses were used to confirm a subject's identity. Those on both lists were deemed to have "broken down." In 1982-3 there were 760 Asian children with a grade 3 or 4 Heaf test response. During the years 1983-7 four subjects were notified as having tuberculosis who had also had a positive Heaf test response in 1982-3. Of these, three were identified on routine radiography performed as a result of the positive test result. Thus prophylactic chemotherapy could have prevented "breakdown" in only one subject over the following four and a half years. We conclude that chemoprophylaxis is not justified in this group.

Neonatal BCG vaccination: its protective effect against childhood tuberculosis

P kelly, J Stinson, L Clancy Peamount Hospital, Newcastle, County Dublin There is a lack of uniformity in policy towards BCG vaccination in Ireland (Irish Med $1988 ; 81: 7)$. To assess the value of neonata BCG vaccination we examined the incidence and spectrum of disease due to tuberculosis in areas which $(a)$ have a policy of neonata BCG; $(b)$ vaccinate at $12-14$ years; $(c)$ never use BCG vaccination (table). We estimate that $\mathbf{8 6}$ cases of tuberculosis were prevented by neonatal BCG vaccination and that 600 800 infants need to be vaccinated to prevent one case of tuberculosis in the under 15 age group in Ireland. We conclude that (1) BCG vaccination protects against tuberculosis in the paediatric ( $<15 \mathrm{y}$ ) age group; (2) because of a relatively high incidence of tuberculosis in Ireland continued use of BCG vaccination in the neonatal period is recommended.

$\begin{array}{lc}\text { Neonatal BCG } & 5 \cdot 33 / 100000^{\star} \\ \text { BCG 12-14 yrs } & 15 \cdot 01 / 100000 \\ \text { No BCG } & 18 \cdot 69 / 100000 \\ \text { No of cases } \leqslant 15 \text { yrs nationally } 77 \text { cases } \\ & 7 \cdot 51 / 100000\end{array}$

${ }^{\star} \mathrm{p}<0.001\left(\chi^{2}\right.$ test $)$

Generation of mycobacterial antigen specific cytotoxic $T$ lymphocytes by tuberculosis patients is related to clinical presentation

AD PITHIE, JA INNES, CJ ELLIS, PB ILES, M RAHELU, DS KUMARARATNE East Birmingham Hospital and Dudley Road Hospital, 
Birmingham Recent animal studies suggest that cytotoxic $T$ lymphocyte (CTL) mediated killing of infected macrophages is important in protective immunity against intracellular bacteria, in particular mycobacteria (Kaufmann. Immunol Today 1988;9:168). We have studied the generation of CTL from the PPD stimulation of peripheral blood mononuclear cells (PBMC) in 33 tuberculosis patients and 22 BCG vaccinated volunteers. Lysis of autologous macrophages by these cells was measured by release of radiolabelled chromium $\left({ }^{51} \mathrm{Cr}\right)$. PPD stimulated CTL from Mantoux positive volunteers show considerable cytotoxic ability, which is maximal after $18 \mathrm{~h}$ incubation. Macrophages which are pulsed first with PPD are significantly more susceptible to lysis than those unpulsed with antigen $(40.3 \%$ and $14.7 \%$ respectively; $p<$ 0.01 ). Mantoux negative controls had significantly less ability to generate CTL ( $p<$ $0 \cdot 01$ ). Generation of antigen specific CTL by tuberculosis patients was related to clinical presentation; those with diffuse non-cavitating disease had relatively poor ability (mean (SD) lysis of PPD pulsed macrophages 18.9 $(7 \cdot 3) \%$ ), while those with cavitating pulmonary or lymph node disease had significantly more ability $(45 \cdot 4(7 \cdot 6) \% ; p<0.01)$. Patients with miliary disease had intermediate ability to generate CTL. The capacity of patients to generate mycobacterial antigen specific CTL may be important in protective immunity against tuberculosis. CTL mediated lysis of infected macrophages, lacking adequate antimycobacterial activity, may expose bacteria to newly recruited and more immunologically active monocytes and therefore contribute to granuloma formation and control of disease. However, uncontrolled lysis of both infected and uninfected macrophages may result in tissue damage and contribute to caseous necrosis and cavitation.

\section{Variations in nebuliser output}

D GRAHAM, K REES, MG PEARSON Regional Thoracic Unit, Fazakerley Hospital, Liverpool Beta agonist administration via jet nebulisers has increased. Physicians specify the drug and the dosage when prescribing but rarely consider the type of nebuliser device. Nebuliser output is known to vary with gas driving pressure, the viscosity of the fluid and temperature. We held these variables constant while assessing the within and between brand variability of nebuliser output. Fifty samples from a single batch of each of three types of jet nebuliser (Cirrus-Intersurgical, System 22-Medicaid and Aeroflow-Unoplast) were studied. Each was filled with $2 \mathrm{ml}$ saline, then weighed before and after nebulisation with $5 \mathrm{l} / \mathrm{min}$ oxygen for two minutes. Subsets of 10 were retested on a second day and then these same subsets were tested again on a third day before and after turning the central jet cone through 90 degrees to assess the effect of acentric positioning of the central jet orifices during mass production. Changing the brand of nebuliser could result on average in changes of $60 \%$ in nebuliser output. However, the aerosol output of individual nebulisers from the same manufacturing batch can vary by over $100 \%$. How these changes are reflected in altered particle size is not known. Overall reproducibility of individual nebulisers was significantly better for each brand (Cirrus $6 \%$, System $229 \%$ and Aeroflow 13\%). However, variation in output of individual nebulisers was con- siderable when jet alignment was changedcirrus fared worst with half the nebulisers varying by over $30 \%$. Thus even when care is taken to specify dosage and air flow rates drug aerosol delivery to the patient can vary widely. Decisions (often taken by non-clinicians) to change hospital suppliers could result in unexpected clinical problems.

\begin{tabular}{|c|c|c|c|}
\hline & \multicolumn{2}{|c|}{ Output ( $\mathrm{ml} / \mathrm{min})$} & \multirow{2}{*}{$\begin{array}{l}\text { Co- } \\
\text { efficient } \\
\text { of varia- } \\
\text { tion }(\%)\end{array}$} \\
\hline & $\begin{array}{l}\text { Mean } \\
\text { (SEM) }\end{array}$ & Range & \\
\hline $\begin{array}{l}\text { Cirrus } \\
\text { System } 22 \\
\text { Aeroflow }\end{array}$ & $\begin{array}{l}0.33(0.04) \\
0.23(0.03) \\
0.19(0.04)\end{array}$ & $\begin{array}{l}0.26-0.42 \\
0.15-0.28 \\
0.13-0.37\end{array}$ & $\begin{array}{l}11 \\
13 \\
22\end{array}$ \\
\hline
\end{tabular}

Humidifier use in patients undergoing long term oxygen treatment (LTOT) using an oxygen concentrator

N PENDLETON, MJ WALSHAW, CRK HIND Department of Medicine, Royal Liverpool Hospital, University of Liverpool A previous study in this district showed that only $46^{\circ}$ of patients prescribed an oxygen concentrator for LTOT were using their machines for the recommended minimum time ( $>15$ h/day) ( $B r$ Med J 1988:297:1030). Among the reasons given by patients for this poor compliance was the complaint of nasal dryness and/or bleeding caused by the therapy, presumably as a result of the low humidity of the oxygen delivered by the concentrator. Humidifier attachments are available to try to increase the humidity of the delivered gas, though it was our clinical impression that few patients were using them. To study this further, all 48 adult patients using an oxygen concentrator within the Liverpool District were interviewed on their use of humidifier attachments using a standard questionnaire. We found that 15 patients $(32 \%)$ had tried humidification at some stage, and that all of these patients were using nasal cannulas. The indications given were: nasal dryness/bleeding ( 11 cases; in five cases this had been recommended by the attending doctor); to simulate the oxygen previously supplied by cyinder (three cases) and humidifier routinely supplied at installation (one case). However, seven patients had stopped using their humidifier attachments because of lack of benefit (four cases), noisy operation (one case), cleaning difficulties (one case), or water collection in the tubing (one case). Of the eight patients still using their humidifier attachments, only three noticed any benefit (all of these had suffered from nasal dryness or bleeding). None of the remaining 33 patients were aware that a humidification facility existed. This study confirms our clinical impression that most LTOT patients are unaware that humidification of oxygen delivered by a concentrator is possible. Furthermore, in those who have tried it only a minority of patients found it to be of any benefit.

Hazards associated with blood gas analysis

PM TWEEDDALE, MB ALLEN, GJR MCHARDY Respiratory Function Laboratory, City Hospital, Edinburgh A short questionnaire on arterial blood gas analysis which included questions on health and safety, was sent to clinical chemistry or cardiorespiratory laboratories in a sample of 95 hospitals in Great Britain. Replies were received from 40 $(42 \%)$. In 25 hospitals unsupervised medical staff were permitted to perform analyses. Formal training was offered to doctors in 22 hospitals, but it was recognised in 15 that self instruction or instruction by medical colleagues took place. Trouble shooting instructions were displayed alongside analysers in 15 of the hospitals. Gloves were provided for users in 22 hospitals but in 10 no special instructions for dealing with high risk samples were provided. Hazards reported by technical staff included syringes submitted with the needle attached in 25 and with the needle resheathed in 23 hospitals. Blood spilt on the analyser or its surroundings was reported in 23 hospitals. In the 15 hospitals where only technical staff performed the analyses, gloves were worn for all analyses in eight. In five hospitals gloves were worn only for high risk specimens but in four of these staff reported that high risk specimens had been submitted without this hazard being indicated. The answers received and the low response rate to the questionnaire suggest that inadequate attention is being given by medical and technical staff to the health and safety aspects of blood gas analysis.

\section{Audit of long term corticosteroid} therapy in a chest clinic

MJ WALSHAW, C BLEASDALE, $S$ WATKIN, CC EVANS Regional Adult Cardiothoracic Unit, Broadgreen Hospital, Liverpool Patients with chronic respiratory diseases are often placed on long term oral steroids, which have potent side effects. Over a four month period we prospectively recorded the way we manage such patients in the outpatient department, with respect to the need for steroids and the monitoring of side effects. Four hundred and eighty nine patients attended a weekly regional chest clinic over the study period, $50(10.2 \%)$ of whom were taking long term oral steroids. These had a mean age of 57 years (range 32-82) and 26 had smoked (mean 30 pack years, range 6-80). Twenty three were females, and 16 of these $(70 \%)$ were postmenopausal. Thirty eight patients had a diagnosis of chronic airflow obstruction; a trial of steroids had initially been carried out in 35 of these and 33 had evidence of reversibility. Ten patients had interstitial lung disease and two bronchiolitis. All were taking prednisolone (mean daily dose $9 \mathrm{mg}$, range 2.5 to 40 ). The mean length of oral steroid use was $6 \frac{1}{2}$ years (range 1 month to 35 years). Forty five patients carried steroid warning cards but only eight increased the dose during other illnesses. Only 19 of those with reversible airflow obstruction increased their steroids during exacerbations. In terms of routine monitoring, only 20 patients were weighed at their clinic visit and 15 had urinalysis. Blood pressure was routinely measured in 26 patients, seven of these by the GP alone and 16 by the chest clinic alone. Significant side effects occurred in 28 patients: 11 had hypertension, one diabetes, four a buffalo hump, four cataracts, six striae, 18 purpura, and five bone problems (three fractures). Most patients also reported weight gain. Review of each patients progress suggested benefit from steroids in 33 cases only. These observations suggest that a significant proportion of patients are on long term oral steroids without deriving benefit, and that side effects are common and not well monitored. 
Intranasal budesonide once daily in seasonal allergic rhinitis

MS BHATIA, LM CAMPBELL, JRM ROSS, MD TAYLOR, EM PEERS, PDI RICHARDSON Astra Pharmaceuticals Limited, King's Langley, Watford Intranasal administration of the glucocorticosteroid budesonide $200 \mu \mathrm{g}$ bid is well established in patients with seasonal allergic rhinitis (Rhinology 1980;18:171) This study evaluated the efficacy of budesonide, in an aqueous base, once daily, a metered dose nasal pump spray being used. In a randomised, double blind parallel group, multicentre trial in the UK, budesonide $400 \mu \mathrm{g}$ mane and $200 \mu \mathrm{g}$ bid were compared in symptomatic seasonal allergic rhinitis patients. Treatment was for three weeks, after a one week run in period. All patients in the $400 \mu \mathrm{g}$ mane group (26M, 21F; mean (SEM) age $28.6(11 \cdot 1) \mathrm{y}$ ) and in the $200 \mu \mathrm{g}$ bid group (27M, 26F; mean (SEM) age 29.0 (13.8) y) had a hay fever history of at least two season (median 7.0 y for both groups). At the final visit the proportions of symptom free patients were: $400 \mu \mathrm{g}$ mane, $40.4 \% ; 200 \mu \mathrm{g}$ bid, $37.7 \%$ (NS, $\chi^{2}$ test). Nasal blockage, running nose, nasal itching and sneezing were rated (0-3 scale) daily by patients, and at entry and follow-up clinic visits. Nasal symptom scores in the two treatment groups, at entry and on completion, did not differ (stratified Wilcoxon test). Nasal symptom scores were related to pollen counts by the pollen interval method (Allergy 1984;39:463); mean symptom scores for pollen quartiles did not differ between treatment groups ( $t$ test). Overall patient assessment of efficacy ( $0-10$ scale) was: $400 \mu \mathrm{g}$ mane, $7 \cdot 8(2 \cdot 1) ; 200 \mu \mathrm{g}$ bid, $7 \cdot 7$ (2.3) (NS, $t$ test). Patients preferred once daily to twice daily treatment $(p<0.001$, sign test). This study suggests that treatmen with aqueous budesonide $400 \mu \mathrm{g}$ mane is as effective as $200 \mu \mathrm{g}$ bid in the treatment of seasonal allergic rhinitis, and is preferred by patients.

Lung function abnormalities following smoke inhalation six months after the King's Cross fire

PW FOGARTY, PJM GEORGE, M SOLOMON, SG SPIRO, RF ARMSTRONG University College Hospital, London Information available on the long term effects of smoke inhalation is confined to firemen with occupational exposure. We describe findings in 14 survivors from the King's Cross underground fire, which occurred on 18 November 1987. There were 11 males and three females. The average age was 36 with a range of 21-55 years. Seven were smokers and seven nonsmokers. Their estimated mean duration of smoke exposure was 80 minutes with a range of one minute to nine hours. Eleven survivors required hospitalisation for periods ranging from one day to six months. Two required endotracheal intubation for laryngeal oedema, one of whom developed ARDS and required ventilation for 24 days. When studied six months after the fire, nine survivors admitted to one or more symptoms, which included hoarseness (one case), cough (five cases), breathlessness (six cases) and worsening of asthma (one case). Dynamic lung volumes were assessed by flow volume loop and static volumes by whole body plethysmography and the results were expressed as percentages of predicted normal values. Mean values for peak expiratory flow, spirometry, total lung capacity, and functional residual capacity were within $20 \%$ predicted. However, $\dot{V}_{50}$ and $\dot{V}_{75}$ were $73 \%$ and $41 \%$ predicted respectively, while residual volume was $149 \%$ predicted. No differences were apparent between smokers and non-smokers. There was no obvious relationship between the survivors' estimated duration of smoke inhalation and the degree of functional impairment. This study shows that significant damage to lung function can persist long term after an acute episode of smoke inhalation.

Implementation of recommendations for a uniform infection control policy: a survey of UK practice

PJV HANSON, SV BAUDOUIN, JV COLLINS Brompton Hospital, London Surveys among bronchoscopists in the UK have revealed a wide diversity of infection control practices apparently resulting from ignorance of the requirements for preventing the spread of HIV, tuberculosis (MTb) and hepatitis B virus (HBV). Recommendations from a working group of the BTS Research Committee have now been published in the society's newsletter (winter 1988) and in the Lancet (1989;ii:270). In a recent questionnaire on bronchoscopic practice in the UK we assessed whether respiratory physicians have changed their infection control precautions. Questionnaires were sent to 310 respiratory physicians in the UK. Forms were completed and returned by $162(52 \%)$. Seventy six per cent of units have at least two fully immersible bronchoscopes. Forty two of 162 units $(26 \%)$ have an automated disinfecting machine, an increase of $8 \%$ from 18 months ago. Seventy five per cent of units still adopt different infection control precautions for patients with MTb, and $67 \%$ do for HIV and HBV. The median duration of disinfection for equipment used on non-infected patients is 20 minutes (range 5-120 minutes). After use on patients with HIV, HBV or MTb the median duration of disinfection is 60 minutes. Glutaraldehyde is used by $153 / 162(94 \%)$ of units; alcohol alone is used by four and Dettox by one; one unit reported not disinfecting the bronchoscope between patients. Fifty of $162(31 \%)$ units have staff who are sensitised to glutaraldehyde-little improvement over the last eight years. We conclude that recommendations on a uniform infection control policy and on reducing glutaraldehyde exposure have not been widely implemented.

Accuracy of oximetry in the investigation of the sleep apnoea-hypopnoea syndrome

MA ALLAN, MF FITZGERALD, J MOLLOY, NJ DOUGlas Rayne Laboratory, Department of Respiratory Medicine, University of Edinburgh Oximetry is being increasingly used in the diagnosis and as a guide to management in patients with the sleep apnoea/hypopnoea syndrome. However, there is a paucity of data comparing the frequency of desaturations with the frequency of apnoeas and hypopnoeas or arousals in such patients. We have therefore examined the frequency of $4 \%$ decreases in oxygen saturation with apnoea plus hypopnoea frequency (Gould. Am Rev Respir Dis 1988;137:895), and with arousal frequency, defining an arousal as a 1.5 second or longer period of return of alpha or theta rhythm associated with increased EMG tone. These relationships were examined in 28 consecutive patients referred with the sleep apnoea/hypopnoea syndrome who had more than 15 apnoeas plus hypopnoeas per hour of sleep. Overall the patients had a mean of 21 (SD) $274 \%$ dips in $\mathrm{SaO}_{2}$ /hour with 39 (24) apnoeas plus hypopnoeas/hour and 21 (12) arousals per hour. There were fair correlations between the number of $4 \%$ dips in $\mathrm{SaO}_{2}$ per hour with apnoea plus hypopnoea frequency $\left(r^{2}=0.72, p<0.001\right)$ and with arousals per hour $\left(r^{2}=0.53, p<0.001\right)$. The mean difference between the number of $4 \%$ dips in $\mathrm{SaO}_{2}$ per hour and apnoeas plus hypopnoeas per hour was - 18 (SD 15), and between $4 \%$ dips and arousals $0.3(20)$. Ten patients had fewer than five falls in $\mathrm{SaO}_{2} /$ hour despite having more than 15 apnoeas plus hypopnoeas per hour, and these patients tended to have a higher baseline saturation $(95 \% v 93 \%, p=0.05)$. Thus the number of $4 \%$ desaturations per hour cannot be directly equated with the number of apnoeas plus hypopnoeas per hour nor with the number of arousals per hour and this must be taken into account in clinical practice.

Cognitive correlates of severity in the sleep apnoea-hypopnoea syndrome

KE CHESHIRE, I DEARY, CM SHAPIRO, NJ DOUGLAS Departments of Psychiatry and Respiratory Medicine, University of Edinburgh There is an association between neuropsychological deficits and hypoxaemia in patients with chronic obstructive pulmonary disease (Fix. Int $J$ Neurosci 1982;16:99). Patients with the sleep apnoea-hypopnoea syndrome report impaired daytime performance, although hitherto this has not been adequately correlated with indices of severity of sleep disturbance or hypoxaemia. Eighteen patients $(16 \mathrm{M}, 2 \mathrm{~F}$; mean age 55 years) with the sleep apnoea-hypopnoea syndrome completed a battery of psychometric tests as did 18 age, sex and educationally matched controls. The patients performed significantly worse than the controls on the Wechsler digit symbol test, a measure of motor persistence, sustained attention, response speed and visuomotor coordination. Patients' test results were also correlated with three measures of illness severity obtained during all night sleep studies: the apnoea-hypopnoea index, frequency of arousals and lowest oxygen saturation levels. When effects due to age were excluded, indices of severity of the sleep apnoea-hypopnoea syndrome correlated positively with poorer test performance on the Wechsler block design and digit symbol tests, with the paced serial addition test, with trail making, and with the simple reaction time $(p<0.05)$. These tests assess functions similar to those of the digit symbol test plus visuospatial organisation, problem solving, and complex conceptual tracking. The apnoea and hypopnoea index correlated most frequently with the severity of psychometric dysfunction. The effect of treatment on these abnormalities can now be studied.

Sleep and breathing pattern in myotonic dystrophy: effect of mazindol

JH COAKLEY, RHT EDWARDS, PMA CALVERLEY University Department of Medicine, Royal 
Liverpool Hospital, Liverpool Patients with myotonic dystrophy (MyD) are known to have sleep disordered breathing (SDB), which has been thought to occur mainly in REM sleep (Coccagna et al. J Neurol Neurosurg Psychiatry 1976). Whether this explains their frequently reported hypersomnulence is unknown. We studied eight patients with a clinical and EMG diagnosis of MyD (age 45 y, FVC 4.3 (0.9) 1). All complained of excess sleepiness on direct questioning but blood gas tensions were normal in all bar one $\left(\mathrm{PaCO}_{2} 6.4 \mathrm{kPa}\right)$. We recorded breathing pattern during EEG confirmed wakefulness and sleep after acclimatisation. Four subjects entered a double blind cross over study of the central stimulant mazindol receiving either placebo or $4 \mathrm{mg}$ of drug orally for four weeks. Central apnoea (CA), obstructive apnoea $(\mathrm{OA})$, and hypopnoea $(\mathrm{H})$ were defined conventionally, as was sleep staging. Baseline results are presented as means (SD) in the table. CA was twice as frequent as $\mathrm{OA}$ and no commoner in REM than when awake. All patients had more than five $\mathrm{A}+\mathrm{H} / \mathrm{hr}$ but SDB severity did not relate to reported symptoms. Breathing abnormality was least in stage $3 / 4$, when compared with wakefulness. Mazindol did not suppress REM but did reduce mean $\mathrm{A}+$ $\mathrm{H}$ from 16 to $6 / \mathrm{h}$. Symptom benefit was greatest in those with most SDB. Irregular breathing is common in $\mathrm{MyD}$, worst in stage 1 with least in 3/4. CA predominates and $O A$ only occurs during sleep. SDB is not easily related to symptoms but can be improved by mazindol.

\begin{tabular}{lrrrl}
\hline $\begin{array}{l}\text { Sleep } \\
\text { stage }\end{array}$ & Min & $C A(\mathrm{No})$ & $O A(\mathrm{No})$ & $A+H / h$ \\
\hline $\mathrm{W}$ & $38(26)$ & 7 & 0 & $18(9)$ \\
1 & $60(34)$ & 20 & 10 & $34(23)$ \\
2 & $131(44)$ & 12 & 3 & $15(12)$ \\
$3 / 4$ & $77(23)$ & 2 & 1 & $4(5)$ \\
REM & $71(40)$ & 16 & 10 & $20(19)$ \\
\hline
\end{tabular}

\section{Value of nocturnal oxygen saturation as} a screening test for sleep apnoea

BG COOPER, D VEALE, CJ GRIFFITHS, GJ GIBSON Departments of Respiratory Medicine and Medical Physics, Freeman Hospital, Newcastle upon Tyne Because of the expense and inconvenience of polysomnographic studies many centres use overnight oxygen saturation $\left(\mathrm{SaO}_{2}\right)$ by oximetry as a screening test in patients with suspected sleep apnoea. We have evaluated recordings of $\mathrm{SaO}_{2}$ in recognising the presence of disorders of breathing during sleep in 46 subjects who were being investigated for possible sleep apnoeahypopnoea syndrome. $\mathrm{SaO}_{2}$ was measured using an ear probe, Biox IIa oximeter and a chart recorder during an "acclimatisation" night immediately prior to a detailed polysomnographic study. The oximetry records were analysed "blind" by two experienced observers; they were classified in three groups: (i) positive: sleep breathing disorder present, (ii) negative: sleep breathing disorder not present, or (iii) uninterpretable: technically unsatisfactory or "don't know." The severity of the condition was graded in terms of the apnoea plus hypopnoea index (AHI) per hour of sleep recorded by polysomnography. The two observers disagreed in the assessment of the oximetry records on $16 / 46$ occasions; in 14 of these cases one or other observer regarded the record as unin- terpretable. Using an $\mathrm{AHI}>5$ as the defining criterion for the presence of sleep apnoea syndrome and regarding inconclusive or uninterpretable records as negative, screening oximetery has a sensitivity of $60 \%$ and a specificity of $96 \%$. If, however, a definition of AHI > 15 per hour is used the sensitivity of oximetry increases to $75 \%$ with a specificity of $88 \%$; the presence of sleep apnoea was confidently recognised from the $\mathrm{SaO}_{2}$ record in the six patients in this series who were most severely affected (AHI 28.7-93.7). We conclude that oximetry is a useful method for the recognition of moderate and severe sleep apnoea. In routine practice, however, an appreciable number of equivocal results is likely, so that repeat oximetry or more detailed polysomnography is required if clinical suspicion is high.

Comparison of two membranes used in the measurement of transcutaneous carbon dioxide during sleep

D CRAMER, M ELLIOTT, S WARD, M BRANTHWAITE Brompton Hospital, London Teflon membranes used with the Hewlett-Packard (HP) Capnometer 47210A for measuring transcutaneous carbon dioxide tensions $\left(\mathrm{TcCO}_{2}\right)$ are no longer manufactured. This expensive equipment is therefore effectively rendered obsolete. Silastic membranes are used in the Corning 178 analyser for measuring arterial $\mathrm{CO}_{2}$ tensions and their use as an alternative to HP membranes has been evaluated. Having established that simultaneous readings from two sites using HP membranes were the same nine patients undergoing routine sleep studies had simultaneous recordings of $\mathrm{TcCO}_{2}$ using the two membranes. Skin preparation was the same at both sites. The Corning membrane was modified by cutting away the thick outer rim so that it could be laid flat upon the skin without wrinkling. A heater and infra-red sensor were then placed upon each membrane and record ings started simultaneously and continued for eight hours. Readings were stored continuously on a BBC "B" microcomputer and values obtained by the two methods were compared at half hourly intervals. The regression equation was $\mathrm{TcCO}_{2}(\mathrm{HP}$ $=0.5+0.998 \quad \mathrm{TcCO}_{2}($ Corn $) \mathrm{mm} \quad \mathrm{Hg}$ $\left(\mathbf{R}^{2}=93 \%\right)$. An analysis of variance between the two methods showed no statistically sig nificant difference $(F=0.05, p=0.83)$. Plotting the differences between the two methods against the average of the two methods showed the differences to be consistent across the range of measurement (45$93 \mathrm{~mm} \mathrm{Hg}$ ). The differences showed a skewed distribution with a median value of $1 \mathrm{~mm} \mathrm{Hg}$ (range +5 to -8 ). We conclude that the Corning membrane accurately reflects changes in transcutaneous $\mathrm{CO}_{2}$ and allows the continued use of equipment which would otherwise be rendered obsolete.

\section{Does nocturnal urinary acid:creatinine} predict nocturnal hypoxaemia?

M ELLIOTT, M BRANTHWAITE Brompton Hospital, London Facilities for investigating suspected nocturnal hypoxaemia are not widely available and a screening test is needed. Correlation between nocturnal hypoxaemia and an overnight increase in the ratio of urinary uric acid to creatinine $(\mathrm{U} / \mathrm{Cr})$ has been demonstrated in patients normoxic by day and not using medication known to influence uric acid ( $A m$ Rev Respir Dis $1987 ; 135: 534)$. The validity of this observation and hence its value as a screening test has been evaluated in a heterogeneous group of patients with suspected hypoxaemia occurring or worsening by night, many of whom were hypoxic by day or were using diuretics. Urine samples were collected on retiring and awakening from 28 patients (16 kyphoscoliosis, five COPD, seven suspected obstructive sleep apnoea) undergoing a routine sleep study. Daytime hypoxaemia was present in 20 and 17 used diuretics regularly. Twenty one patients breathed spontaneously and seven were ventilated mechanically overnight using the nasal route. The change in $\mathrm{U} /$ $\mathrm{Cr}$ was calculated using the formula $\Delta \mathrm{U} /$ $\mathrm{Cr}=[\mathrm{U} / \mathrm{Cr}(\mathrm{am})-\mathrm{U} / \mathrm{Cr}(\mathrm{pm})] \mathrm{U} / \mathrm{Cr}(\mathrm{pm})$

$\times 100 \%$. Oxygen saturation was recorded continuously using an Ohmeda Biox III pulse oximeter and a desaturation score calculated as $\Sigma\left[\left(90-\mathrm{SaO}_{2}\right) \times \mathrm{T} / \mathrm{T}\right.$ tot $\left.\times 100 \%\right]$, where $\left(90-\mathrm{SaO}_{2}\right)$ was the magnitude of the desaturation below $90 \%$ and $\mathrm{T} / \mathrm{T}$ tot was the proportion of time spent at that saturation. A score greater than 20 was considered significant. Significant desaturation was recorded in 19 studies (median score 117 , range $22 \cdot 5-1135$ ) The median change in the overnight $\mathrm{U} / \mathrm{Cr}$ ratio was $-81 \%$ (range -47 to $+80 \%$ ) in the hypoxaemic patients and $-6 \cdot 7 \%$ (range -96 to +21 ) in the non-hypoxaemic group. There was no correlation between nocturnal desaturation and overnight change in $\mathrm{U} / \mathrm{C}$ ratio ( $r$ squared adjusted $1.5 \%$ ). It is concluded that the overnight change in urinary $\mathrm{U} / \mathrm{Cr}$ ratio is of no value in predicting the occurrence or severity of nocturnal desaturation in patients with daytime abnormalities of gas exchange or requiring diuretic therapy.

Nocturnal hypoxaemia and polycythaemia in patients with extrapulmonary restrictive disorders

MW ELLIOTT, JA WEDZICHA, MA BRANTHWAIT Brompton Hospital, London Intermitten hypoxia has been shown to cause right ventricular hypertrophy and polycythaemia in rats (Clin Sci 1985;69:595). Nocturnal hypoxaemia has been postulated as the cause of otherwise unexplained pulmonary hypertension and polycythaemia in patients with COPD and obstructive sleep apnoea. Patients with chest wall deformity and neuromuscular disease often have profound nocturnal desaturation with relatively well preserved arterial oxygen tensions by day. Pulmonary arterial hypertension is common in these patients but polycythaemia is seldom seen clinically. A retrospective study of all sleep studies performed over the last two years revealed 17 patients not previously treated with oxygen or mechanical ventilation who had profound nocturnal arterial oxygen desaturation. The median percentage of night with saturation less than $90 \%$ was $87.5 \%$ (range $35-100 \%$ ). Fourteen patients had no evidence of polycythaemia, mean haemoglobin $14.5 \mathrm{~g} / \mathrm{dl}$ (SD 1.7), mean packed cell volume 0.45 (SD 0.05), despite considerable nocturnal hypoxaemia. The mean daytime $\mathrm{PaO}_{2}$ was $8.5 \mathrm{kPa}$ (SD 1.4 ) and $3 / 14$ had daytime oxygen saturation $<90 \%$. Three patients showed mild elevation of $\mathrm{Hb}(15 \cdot 1$ $18,17.7 \mathrm{~g} / 1)$ and/or packed cell volume $(0.54$, $0.54,0.52)$. All three were taking diuretics and were hypoxic by day $(4 \cdot 8,6 \cdot 9,7 \mathrm{kPa})$ None of the patients were current smokers 
and most were hypercapnic (mean $\mathrm{PaCO}_{2} 7$ $\mathrm{kPa}(\mathrm{SD} \mathrm{0.8)}$; these may be important factors in explaining the surprisingly low incidence of polycythaemia. Further prospective studies in this group of patients may be helpful in elucidating the role of nocturnal hypoxaemia as a cause of polycythaemia.

Sleep quality and daytime cognitive performance in nocturnal asthma

MF FITZPATRICK, K CHESHIRE, KF WHYTE, I DEARY, CM SHAPIRO, NJ DOUGLAS Departments of Respiratory Medicine and Psychiatry, University of Edinburgh Patients with nocturnal asthma suffer significant sleep disruption (Catterall JR, et al. Lancet 1982;i:301). In order to elucidate whether this sleep disruption might lead to demonstrable impairment in daytime cognitive performance, we studied nine patients with nocturnal asthma (NA), defined as an at least $15 \%$ overnight fall in peak flow rate plus waking with respiratory symptoms at least once per week, nine age and intellect matched normal subjects (NL), and nine age matched asthmatics (A) taking regular drug therapy who did not have nocturnal asthma. All subjects had two consecutive overnight sleep studies for objective assessment of sleep quality and a multiple sleep latency test (Carskadon MA, et al. Sleep 1986;9:519) and completed a programme of psychometric tests. Patients with nocturnal asthma performed less well than either of the other two groups on two tests, trail making (joining a sequence of numbers in a complex spatial arrangement) and the paced serial addition test (PASAT). Trail making times (mean (SD), seconds) were: NA 95 (32), A 60 (23), NL 62 (16); p < 0.02, ANOVA. PASAT scores (number of correct additions out of 60) were: NA 34 (12), A 42 (5), NL 47 (9); $\mathrm{p}<0.01$, ANOVA. There were no significant differences between the three groups in any of the other psychometric tests or in multiple sleep latency tests. We conclude that patients with nocturnal asthma do not have any objective evidence of daytime sleepinesss but do have impaired daytime cognitive performance of a magnitude which might affect performance at work. We are unable, as yet, to discern whether this impairment is a result of sleep disruption or their drug therapy (six patients with NA and three with A were taking oral theophyllines).

\section{Comparison of four nasal CPAP sys- tems}

AH KENDRICK, JR CATTERALL Respiratory Department, Bristol Royal Infirmary, Bristol Several commercial nasal CPAP systems are now available for treatment of the sleep apnoea syndrome, but we are aware of no work comparing them. We have compared four devices: (1) Sefam Mini Pression Plus, single (1a) and double (1b) tube system; (2) Thomas Respiratory Systems; (3) Respironics Sleepeasy II; and (4) ACI Medical-Systems. Measurements were made on four patients $(3 \mathrm{M}, 1 \mathrm{~F} ; 27-58 \mathrm{y})$ with documented sleep apnoea syndrome and included $(a)$ sound level (dB); (b) flow rate from the pump $(1 / \mathrm{s}) ;(c)$ pressure range $\left(\mathrm{P}\right.$ range, $\left.\mathrm{cm} \mathrm{H}_{2} \mathrm{O}\right)$ at the nose; $(d)$ relative humidity $(\%)$ and temperature $\left({ }^{\circ} \mathrm{C}\right)$ inside the nasal mask; and $(e)$ inspired and end tidal $\mathrm{CO}_{2}$ and $\mathrm{O}_{2}$ concentrations (\%) in the mask. Measurements were

\begin{tabular}{|c|c|c|c|c|c|}
\hline Device & $1 a$ & $1 b$ & 2 & 3 & 4 \\
\hline $\begin{array}{l}\text { Sound level } \\
\text { Flow rate } \\
\text { Temperature } \\
\text { Humidity } \\
\text { P range } \\
\text { FETO }_{2} \\
\mathrm{FIO}_{2} \\
\mathrm{FEICO}_{2} \\
\mathrm{FICO}_{2} \\
\end{array}$ & $\begin{array}{l}51 \\
1.2 \\
31 \cdot 2(0.2) \\
84 \\
2 \cdot 1-17 \cdot 3 \\
16 \cdot 4(0.4) \\
20.2(0.2) \\
4 \cdot 49(0.4) \\
0.80(0.3)\end{array}$ & $\begin{array}{l}51 \\
1 \cdot 2 \\
31 \cdot 3(0.2) \\
85 \\
2 \cdot 2-16.9 \\
16 \cdot 1(0.4) \\
20 \cdot 2(0.2) \\
4 \cdot 46(0.3) \\
0.49(0.4)\end{array}$ & $\begin{array}{l}84 \\
2.67 \\
27.9(0.3) \\
84 \\
2.5-16.9 \\
17.2(0.4) \\
20.4(0.2) \\
5.07(0.5) \\
0.18(0.2)\end{array}$ & $\begin{array}{l}60 \\
2 \cdot 00 \\
30 \cdot 7(0 \cdot 2) \\
81 \\
2 \cdot 1-16 \cdot 1 \\
16 \cdot 6(0 \cdot 4) \\
20 \cdot 4(0.2) \\
4 \cdot 37(0 \cdot 7) \\
0.37(0.3)\end{array}$ & $\begin{array}{l}70 \\
4 \cdot 33 \\
31 \cdot 1(0 \cdot 3) \\
66 \\
1 \cdot 5-20 \cdot 3 \\
16 \cdot 3(0 \cdot 5) \\
20 \cdot 5(0 \cdot 2) \\
5 \cdot 15(0 \cdot 6) \\
0 \cdot 16(0 \cdot 1)\end{array}$ \\
\hline $\begin{array}{l}\text { Cost }(f): \\
\text { Capital } \\
\text { Annual }\end{array}$ & $\begin{array}{l}750 \\
13.40\end{array}$ & $\begin{array}{l}750 \\
13.40\end{array}$ & $\begin{array}{l}650 \\
111 \cdot 70\end{array}$ & $\begin{array}{l}790 \\
10.60\end{array}$ & $\begin{array}{l}560 \\
28 \cdot 30\end{array}$ \\
\hline
\end{tabular}

made over $40-60$ breaths after $30-60$ minutes acclimatisation with each system. The table gives results as means (SD) of all breaths. The capital outlay includes mask and tubing and the annual running costs are based on eight hours use per night. The end tidal gas concentrations did not alter despite increasing pressure. The main differences between the devices were in flow rate, humidity of the inspired air, sound level and running costs. Despite differences in flow rate, $\mathrm{CO}_{2}$ retention did not occur with any device and the double tube system had no obvious advantage over the single tube system.

\section{Pharyngeal function in patients with OSA}

S WRIGHT, N ZAMEL, $\mathbf{v}$ HOFFSTEIN Royal Victoria Hospital, Belfast, and Department of Respiratory Medicine, University of Toronto, and St Michael's Hospital, Toronto. Previous investigations have indicated that patients with obstructive sleep apnoea have abnormal pharyngeal function even when measured in the awake state. We studied 94 unselected patients referred to a sleep clinic because of snoring and a suspicion of sleep apnoea, to assess two different measurements of pharyngeal function: (1) changes in pharyngeal area with lung volume (PLVD-pharyngeal lung volume dependence), and (2) changes in pharyngeal area with externally applied positive pressure (COMP-pharyngeal compliance). All patients had pharyngeal areas measured by the acoustic reflections technique at functional residual capacity $\left(\mathrm{A}_{\mathrm{FRC}}\right)$, residual volume $\left(A_{R V}\right)$, and at FRC after a $4 \mathrm{~cm} \mathrm{H}_{2} \mathrm{O}$ positive pressure was applied to the pharynx $\left(\mathrm{A}_{4}\right)$. From these measurements we calculated PLVD $\left(\mathrm{A}_{\mathrm{FRC}}-\mathrm{A}_{\mathrm{RV}} / \mathrm{ERV}\right.$, where $\mathrm{ERV}=$ expiratory reserve volume) and $\operatorname{COMP}\left(\mathrm{A}_{4}-\mathrm{A}_{\mathrm{FRC}} / \Delta \mathrm{P}\right.$, where $\Delta \mathrm{P}=4 \mathrm{~cm}$ $\mathrm{H}_{2} \mathrm{O}$ ). On the basis of the results of nocturnal polysomnography we divided the patients into two groups: 59 apnoeics (apnoea/ hypopnoea index (AHI)>10) and 35 nonapnoeics $(\mathrm{AHI}<10)$. We found no significant difference in age, weight or body mass index (BMI) between the two groups or in pharyngeal size or COMP. However, PLVD in the apnoeic patients $(1.5 \pm 2.5)$ was significantly higher than in the non-apnoeic patients $(1.1$ $\pm 1.5, p<0.01)$. Linear regression analysis on all 94 patients revealed that only BMI, age and PLVD were significant determinants of AHI. We conclude that PLVD (1) is significantly higher in apnoeic than in nonapnoeic snorers; (2) is a significant determinant of AHI; and (3) is not correlated with pharyngeal compliance. Finally, although PLVD and COMP both may reflect pharyngeal function, they are independent measurements, possibly determined by different mechanism.
Cephalometric measurements in erect and supine postures on patients with sleep apnoea

N YILDIRIM, MF FITZPATRICK, R JALLEH, AJ Wightman, NJ DOUglas Departments of Respiratory Medicine and Radiology, University of Edinburgh Cephalometric measurements taken in the erect position are often used to describe upper airway anatomy in patients with obstructive sleep apnoea (OSA) (Partinen $\mathrm{M}$ et al. Chest 1988;93: 1199). However, OSA is caused by dynamic upper airway obstruction during sleep, usually in the supine position. We have therefore performed 23 cephalometric measurements on each of 29 patients with OSA in both erect $(E)$ and supine $(S)$ postures. There were no differences in any of the bony dimensions between erect and supine postures. However, the airspace behind the uvula became much narrower in the supine posture: uvula-pharyngeal wall (mandibular plane) (mean (SD) mm) (E) 5.1 (2.2), (S) 3.5 $(3.0), \mathrm{p}=0.01$, with the uvula becoming thicker, (E) 11.7 (2.6), (S) 13.0 (2.6), p = 0.002 . At the same time, the posterior airways space (behind the tongue and below the uvula) widened in the supine posture: (E) 11.5 (4.5), (S) 13.4 (4.8), p = 0.003; with widening also of the distance between hyoid and pharyngeal wall: (E) $39.5(6.4),(S) 41.3$ $(6.3), p=0.01$. The angle of the neck (between anterior wall of $\mathrm{C} 2$ and $\mathrm{C} 4$ ) was held in a position of greater flexion in the supine posture: (mean (SD) degrees) (E) 19 (11), (S) $14(8), p=0.0001$. We conclude that the supine posture has a substantial effect on upper airway dimensions in patients with OSA, predisposing to obstruction at the level of the uvula.

Systolic blood pressure changes reflect inspiratory effort during simulated obstructive apnoea

S LEA, N ALI, M GOLDMAN, L LOH, J FLEETHAM, J STRADLING Osler Chest Unit, Churchill Hospital, Oxford We have measured changes in systolic blood pressure ( $\triangle \mathrm{SBP}$ ) by using a continuous non-invasive monitor of the arterial pressure waveform (Finapres, Ohmeda 2300) during simulated obstructive age 27, range 17-45 y). Subjects made repeated inspiratory efforts at FRC against an 5 seconds, until unable to maintain the breath hold. Mouth pressure behind the obstruction was recorded and could be seen by the subject. During each run they were asked to generate a fixed negative pressure with as "square wave" a profile as possible. This was done at six different pressures $(-5,-10$, sleep apnoea in 11 healthy volunteers (mean obstructed mouthpiece for 2.5 seconds every 
$-20,-30$ and $-40 \mathrm{~cm} \mathrm{H}_{2} \mathrm{O}$ ) in random order with a control run of just breath hold alone. Each run averaged about six breaths and three runs at each pressure were performed. SBP reflected mouth pressure changes $(\triangle M P)$ and therefore by inference changes in pleural pressure since no flow was generated during the inspiratory efforts. The relationship between $\triangle \mathrm{SBP}$ and $\triangle \mathrm{MP}$ was closest at small $\triangle \mathrm{MPs}$. At higher $\triangle \mathrm{MPs}$ (over $20 \mathrm{~cm} \mathrm{H}_{2} \mathrm{O}$ ) the slope of $\Delta \mathrm{SBP}$ versus $\Delta \mathrm{MP}$ flattened so that only about $73 \%$ (range 50 $100 \%$ ) of the swings in MP were reflected in SBP swings. During tidal breathing synchronous swings of about $6 \mathrm{~mm} \mathrm{Hg}$ were observed (which disappeared during breath holds with no inspiratory effort) and presumably reflected the pleural pressure swings of quiet ventilation. Non-invasive monitoring of beat by beat SBP provides a record of respiratory rate and a qualitative estimate of respiratory effort (swings in pleural pressure). Thus this device in conjunction with an oximeter may be useful in monitoring sleep apnoea and, by estimating respiratory effort, differentiate between the obstructive and central variants.

Effect of intravenous atrial natriuretic peptide on bronchomotor tone in normal subjects

G HULKS, A JARDINE, JMC CONNELL, NC THOMSON Department of Respiratory Medicine and MRC Blood Pressure Unit, Western Infirmary, Glasgow We have previously demonstrated that the intravenous infusion of atrial natriuretic peptide (ANP) is able to produce marked bronchodilation in asthmatic subjects and wished to see if a similar response could be seen in normal subjects. Eight (2F) non-asthmatic subjects (mean age $31.6 \mathrm{y}$ ) were studied, each of whom had previously shown $\geqslant 35 \%$ increase in specific airways conductance (sGaw) to inhaled salbutamol. Either ANP or placebo were given by continuous intravenous infusion in a randomised, double blind crossover manner on two study days. Doses of ANP corresponding to $0.5,2.0$ and $10 \mathrm{pmol} /$ $\mathrm{kg} / \mathrm{min}$ were given for 20 minutes, each in incremental fashion. Plasma, ANP, catecholamines, pulse, blood pressure and sGaw were measured prior to infusion and at the end of each infusion period. Mean (SEM) baseline sGaw was similar on the two study days: $0.24(0.04)\left(\mathrm{s}^{-1} \mathrm{kPa}^{-1}\right)$ and $0.215(0.03)$, placebo and ANP respectively (see table above). Having been unable to demonstrate a significant bronchodilator response at those doses, we wished to determine whether alteration of airway calibre could be achieved at any dose. A second study was therefore performed on six of the eight original subjects, who received sequential 20 minute infusions of placebo, ANP ( $50 \mathrm{pmol} / \mathrm{kg} / \mathrm{min}$ ) and then placebo again in a single blind manner; sGaw changed by $0.3(3.5) \%, 31$ $(9.5) \%$ and $-1.7(7 \cdot 2) \%$ with successive infusions ( $p<0.01$ ANP $v$ placebo). During active infusion plasma ANP rose to 2056 (154) $\mathrm{pg} / \mathrm{ml}$. These studies demonstrate that at pathophysiological plasma levels ANP produces no significant bronchodilation in normal subjects. At pharmacological levels, however, a marked response can be seen.

$\mathrm{GH}$ was supported by a Wellcome medical graduate fellowship.

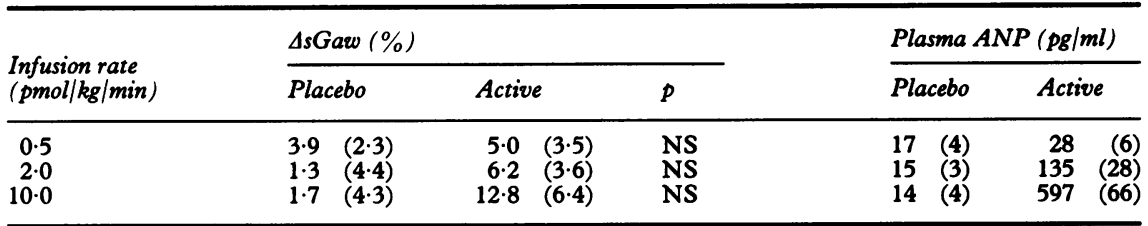

Response of atrial natriuretic peptide to exercise in asthmatic subjects

G HULKS, AF MOHAMMED, A JARDINE, JMC CONNELL, NC THOMSON Department of Respiratory Medicine and MRC Blood Pressure Unit, Western Infirmary, Glasgow Atrial natriuretic peptide (ANP) is known to rise in normal individuals in response to exercise. Having previously demonstrated that elevated plasma levels of ANP may produce significant bronchodilation in asthmatic subjects, we wished to determine whether their ANP response to exercise was the same as normal subjects. The subjects were eight men, mean (SEM) age $32 \cdot 1$ (3.2), with a previously documented fall of $\geqslant 20 \% \mathrm{FEV}_{1}$ in response to exercise, and a control group of eight age matched non-asthmatic males. Each subject underwent graded treadmill exercise to exhaustion according to the Bruce protocol, during which time heart rate and minute oxygen consumption $\left(\dot{\mathrm{VO}}_{2}\right)$ were continually monitored. Prior to the commencement of exercise, and during the final 30 seconds of each subsequent stage, $15 \mathrm{ml}$ blood were withdrawn via an indwelling intravenous cannula for the estimation of plasma ANP. $\mathrm{FEV}_{1}$ was recorded before exercise and at 2,5 , 10 and 20 minutes after completion, at which times samples were again withdrawn for peptide estimation. There was a $>3$ fold increase in ANP post-exercise in each group but we were unable to demonstrate any significant difference between the two groups. These results suggest that aberrations of the ANP response are not instrumental in the aetiology of exercise induced asthma.

GH was supported by a Wellcome medical graduate fellowship.
ANP were taken with the subject recumbent at a time when the patients were clinically stable. Assay was performed in a single batch following preliminary florisil extraction and by an established radioimmunoassay. Spirometry, 12 minute walk and estimation of arterial blood gas tensions were performed within one week of venesection. All subjects were hypoxaemic (mean (SD) $\mathrm{Po}_{2} 7 \cdot 35$ (1.55) $\mathrm{kPa}$ ) and five subjects had $\mathrm{PCO}_{2}$ greater than $7 \mathrm{kPa}$. Mean FEV, was $0.59(0.29) 1$ and FVC was $1.46(0.51) 1$. Mean 12 minutes walking distance was 537 (203) m. Mean plasma ANP was $13.6(6.7) \mathrm{pmol} / \mathrm{l}(\mathrm{NR}>15 \mathrm{pmol} / \mathrm{l})$. There was no correlation between the blood gases, ANP, and physiological variables. We have previously shown elevation of plasma ANP in primary pulmonary hypertension (Thorax 1987;42:720), but were unable to exclude hypoxia as a mechanism of release in these patients. These data demonstrate that respiratory failure per se does not cause elevation of plasma ANP.

The ANP assay is supported by the British Lung Foundation. We thank JMS, JES, TWH and JW for permission to study their patients.

Atrial natriuretic peptide release in obstructive sleep apnoea

ID PAVORD, D SPENCE, $S$ DUTT, RF BING, P EBDEN Department of Medicine, Glenfield General Hospital, Leicester Obstructive sleep apnoea (OSA) is characterised by repeated upper airways occlusion and arterial desaturation during sleep. A nocturnal diuresis and natriuresis has been observed in patients with OSA, which tends to normalise when

\begin{tabular}{|c|c|c|c|c|}
\hline \multirow[b]{3}{*}{$\begin{array}{l}\text { Stage } \\
\text { Baseline } \\
1 \\
2 \\
3 \\
4 \\
5 \\
2 \text { min post } \\
5 \text { min post } \\
10 \text { min post } \\
20 \text { min post }\end{array}$} & \multicolumn{2}{|c|}{ Plasma ANP (pg/ml) } & \multicolumn{2}{|c|}{$\triangle F E V,(\%)$} \\
\hline & Control & Asthmatic & Control & Asthmatic \\
\hline & $\begin{array}{ll}20 & (4) \\
15 & (2) \\
16 & (2) \\
22 & (2) \\
37 & (8) \\
45 & (8) \\
69 & (12) \\
66 & (10) \\
53 & (11) \\
41 & (8)\end{array}$ & $\begin{array}{lr}20 & (5) \\
16 & (2) \\
19 & (3) \\
26 & (4) \\
39 & (10) \\
57 & (14) \\
63 & (11) \\
61 & (17) \\
44 & (9) \\
31 & (5)\end{array}$ & $\begin{array}{ll}3 & (2) \\
4 & (1) \\
3 & (2) \\
2 & (2)\end{array}$ & $\begin{array}{ll}-19 & (4) \\
-22 & (3) \\
-22 & (4) \\
-21 & (6)\end{array}$ \\
\hline
\end{tabular}

Plasma concentrations of atrial natriuretic peptide in adult cystic fibrosis

AH MORICE, K THOMPSON, A ROSSER Chest Medical Unit, Papworth Hospital, Cambridge Severe hypoxia in vitro has been shown to cause release of atrial natriuretic peptide (ANP). Adult cystic fibrosis (CF) is characterised by severe bronchiectasis and arterial hypoxaemia. We have investigated 21 patients, aged 18-37 years, with proved CF to determine whether the disturbance of lung mechanics or blood gas tensions seen in this condition causes an increased secretion of ANP. Venous plasma samples for assay of treated with continuous positive airways pressure (CPAP). The mechanism may be atrial natriuretic peptide (ANP) release secondary to atrial distension. This may occur during the large negative intrathoracic pressure observed in OSA or as a result of raised right heart pressure due to hypoxaemia. Previous studies have shown conflicting results. Recently Kreiger et al (Clin Sci 1989;76:31) have shown significantly higher urinary guanosine $3^{\prime}, 5^{\prime}$-cyclic monophosphate excretion (suggesting raised ANP activity) in patients with OSA off CPAP than on CPAP. We have measured plasma ANP levels during sleep in five patients with moderate, symptomatic OSA. Seven blood 
samples were taken between 1 and 2 am on both OSA and CPAP nights. ANP was assayed using the Amersham radioreceptor assay. All subjects showed obstruction and arterial desaturatin (mean fall in $\mathrm{O}_{2}$ saturation $12 \%$ ) before and during sampling on the OSA night. CPAP was successful in abolishing obstructive episodes in all subjects on the CPAP night. Higher mean plasma ANP levels were found on OSA nights than on CPAP nights (31.0 (SEM 9.8) $v 19.7$ (9.6) $\mathrm{pmol} / 1$ respectively; $\mathrm{p}<0.05$ ). The effect was observed in all five subjects. Mean overnight urinary sodium excretion was higher on OSA nights $(53.8 \mathrm{mmol})$ than CPAP nights $(32.8$ mmol). These results confirm that plasma ANP is raised in OSA during periods of obstruction and is corrected by treatment with CPAP. This provides an explanation for the natriuresis and diuresis observed in this condition.

Atrial natriuretic peptide levels during intravenous saline infusion in hypoxic cor pulmonale

PA BARDSLEY, AG STEWART, SV BAUDOUIN, JC WATERHOUSE, AH MORICE, P HOWARD University Department of Medicine, Royal Hallamshire Hospital, Sheffield The pathogenesis of the oedematous state in hypoxic cor pulmonale is poorly understood. A failure of atrial natriuretic peptide (ANP) release, leading to salt and water retention is a possible mechanism. Plasma ANP levels were measured during and for three hours after an intravenous saline load $(0 \cdot 1 \mathrm{ml} / \mathrm{kg} / \mathrm{min} 2 \cdot 7 \%$ saline over $60 \mathrm{~min}$ ) in 14 patients with COAD. Seven patients had cor pulmonale, typical "blue bloaters" with previous acute exacerbations associated with peripheral oedema (mean age 69 (SEM 5) y, FEV 10.7 $(0.1) 1, \mathrm{PaO}_{2} 6.6(0.5) \mathrm{kPa}$ and $\mathrm{PaCO}_{2} 6.7(0.4)$ $\mathrm{kPa}$ ) and seven control patients had the "pink and puffing" form of COAD (age 64 (1) $y$, FEV 1 1.1 (0.1) 1, $\mathrm{PaO}_{2} 9 \cdot 2(0.5) \mathrm{kPa}$ and $\mathrm{PaCO}_{2}$ $5 \cdot 1(0.3) \mathrm{kPa}$ ) with no previous episodes of oedema. All patients were studied fasting and after diuretics had been stopped for three days. No supplemental oxygen was given. Mean four hourly urine sodium excretion was $31(10 \mathrm{mmol}$ ) (NS), $46 \%$ of the load (NS) in the controls. Initial mean plasma ANP values were higher in the cor pulmonale patients, $20.4(1.4)$ and $10.0(1.5) \mathrm{pmol} / 1$ respectively $(\mathrm{p}<0.01)$ and the mean peak rises in ANP were $18.7(8.0) \mathrm{pmol} / \mathrm{l}$ in the cor pulmonale patients and 9.1 (2.3) pmol/1 in the controls NS). There were no significant differences in plasma and urinary osmolality, blood pressure or creatinine clearance between the groups. In conclusion, the previously described impaired ability of patients with hypoxic cor pulmonale to excrete an intravenous saline load is not explained by a lack of ANP secretion and probably reflects defective renal sodium handling.

We thank the British Lung Foundation for their support of the ANP assay.

Phrenic nerve cold injury: cause of diaphragm paralysis following open heart surgery

J EFTHIMIOU, AB HASSAN, CH WOODHAM, S WESTABY, MK BENSON John Radcliffe and Churchill Hospitals, Oxford Diaphragm paralysis, usually unilateral and left sided, is increasingly recognised following open heart surgery and occurs in between 30 to $75 \%$ of patients, as determined radiologically (Large et al. Thorax 1985;40:444; Esposito and Spencer. Ann Thorac Surg 1987;43:503). Phrenic nerve cold injury due to the use of ice/slush topical hypothermia has been suggested as the cause, although other mechanisms such as surgical trauma have also been implicated. We studied 100 consecutive patients undergoing open heart surgery in our unit, half of whom received ice/slush topical hypothermia (group 1) and half of whom did not (group 2). The two groups were similar in terms of age and sex. Twenty eight $(56 \%)$ group 1 patients and $34(68 \%)$ group 2 patients had valve replacements, $18(36 \%)$ group 1 and $11(22 \%)$ group 2 patients had coronary artery bypass grafts and four $(8 \%)$ group 1 and five (10\%) group 2 patients had atrial septal defect repairs. Aortic cross clamp time was less in group 1 (61.5 (SD 15.6) $\mathrm{min}$ ) compared with group $2(74.4(20.8) \mathrm{min})$, although this difference was not significant. Significant differences, however, were found for radiological evidence of partial left lower lobe collapse $(82 \% v 32 \%, \mathrm{p}<0.01)$ and for radiological evidence of diaphragm paralysis within the first postoperative week $(36 \% v$ $2 \%, \mathrm{p}<0.001)$. Eighteen group 1 patients developed unilateral diaphragm paralysis $(17$ left sided, one right sided) compared with only one patient in group 2 . In these 18 group 1 patients diaphragm paralysis was present in $13(26 \%)$ at one month and in seven $(14 \%)$ at one year postoperatively. There were no significant differences between the two groups in terms of postoperative arrhythmias, myocardial infarction or mortality. The use of ice/slush topical hypothermia during open heart surgery is associated with a high incidence of diaphragm paralysis and its myocardial protective value could be more selectively applied.

\section{Bronchodilation detected by the inter-} rupter technique

R JOHNSON, PJ CHOWIENCZYK, $S$ LANE, GM COCHRANE, N WILSON, M SILVERMAN Guy's Hospital and Royal Post Graduate Medical School, Hammersmith Hospital, London The interrupter method for measuring airway resistance (Rint) has important practical advantages. It does not require cooperation by the patient and can thus be used in young children or older subjects, unable to cooperate with conventional methods. We have recently described a new portable instrument for measuring Rint, a new device for measuring airway resistance based on the interrupter technique (Eur Respir $J$ 1989;2:648). We have investigated the sensitivity of this new device in detecting bronchodilation. In 25 subjects (17 adults and eight children) with reversible airways obstruction pre and post bronchodilator measurements of $R$ int were compared with measurements of $\mathrm{FEV}_{1}$. In the adults changes in Rint were also compared with changes in airway resistance measured using the body plethysmograph (Raw). A $20 \%$ or greater change in $F E V_{1}$ postbronchodilator was seen in only $5 / 25$ patients whereas a similar change in Rint was obtained in 19/25 patients. Changes in Rint were significant in $21 / 25$ patients $(p<0.05)$. A significant change in Raw was seen in 16 of the 17 adults A significant change in Rint was seen in all 17 adults. The mean percentage change in Rin $\left(37^{\circ}\right)$ was not significantly different from the mean percentage change in Raw (39\%). During an ongoing study to investigate bronchial hyperresponsiveness in 3 year olds we observed an increase in Rint following methacholine and a decrease following bronchodilator in $10 / 10$ subjects.

Placebo controlled comparison of the bronchodilator performance of salmeterol and salbutamol over 12 hours

G BOYD, K ANDERSON, R CARTER Department of Respiratory Medicine, Glasgow Royal Infirmary, Glasgow The efficacy and duration of action of salmeterol hydroxynaphthoate $50 \mu \mathrm{g}$ administered both as dry powder by Diskhaler and by metered dose inhaler were studied in 11 asthmatic patients and compared with salbutamol aerosol $200 \mu \mathrm{g}$ and placebo over a 12 hour period. The patients (five male, six female; aged 23-69 years, median 45), who all demonstrated at least $15 \%$ reversibility to inhaled salbutamol 200 $\mu \mathrm{g}$, were studied on four different days using a double blind, double dummy crossover technique. All inhaled bronchodilator therapy was withheld for eight hours prior to the study and, after baseline measurement of $\mathrm{FEV}_{1}$, the study medication was given and $F E V_{1}$ recorded every 15 minutes for one hour and hourly thereafter up to 12 hours. Rescue bronchodilator was provided as required. In terms of the onset of action of the drugs as assessed by $15 \%$ increase in $\mathrm{FEV}_{1}$, the median response for salbutamol $(7 \mathrm{~min}$ ) was quicker but not significantly so than salmeterol dry powder ( $11 \mathrm{~min}, \mathrm{p}=0.07)$ but significantly quicker than salmeterol aerosol (14 min, $\mathrm{p}=0.04)$. Peak levels of bronchodilation were the same for all active treatments (salmeterol 2.25 1 (SE 0.236) and salbutamol 2.21 1 (0.231). Salbutamol produced a greater bronchodilator effect than placebo for up to five hours (mean FEV 1.89 (0.28); $\mathrm{p}=0.006)$; however, salmeterol aerosol and dry powder produced significant bronchodilation at 12 hours (mean $\mathrm{FEV}_{1} 1.89$ $1(0.163), p=0.03)$. Salmeterol aerosol and dry powder produce significant bronchodilation for 12 hours compared with only five hours following salbutamol and this longer duration of action may afford greater control of asthma when given as a twice daily treatment.

Large volume spacer devices can reduce hypothalamo-pituitary-adrenal axis suppression associated with high dose beclomethasone dipropionate

PH BROWN, AP GREENING, GK CROMPTON Department of Respiratory Medicine, Northern General Hospital, Edinburgh High doses of beclomethasone dipropionate (BDP) may be associated with hypothalamo-pituitaryadrenal (HPA) axis suppression. From a survey of HPA function in 53 adult asthmatics taking 1500-2500 $\mu \mathrm{g}$ BDP daily via metered dose aerosol, 12 patients with significant HPA suppression were identified. HPA function tests performed were 9 am serum cortisol, short tetracosactrin test and $24 \mathrm{~h}$ urine free cortisol excretion. Subnormal test results were defined as 9 am serum cortisol $<190 \mathrm{nmol} / \mathrm{l}$; rise in serum cortisol following tetracosactrin $<200 \mathrm{nmol} / 1$ and/or achieved serum cortisol $<500 \mathrm{nmol} / 1$; urine free cortisol excretion $<80 \mathrm{nmol} / 24 \mathrm{~h}$. Significant HPA suppression was judged to be 
present if results of at least two tests were subnormal. Of the 12 patients $(7 F, 5 M)$, five had previously required long term prednisolone, discontinuing its use 3.6-10 years prior to assessment. Only one patient had required a short course of prednisolone in the preceding three months. Ten of these 12 patients were instructed in the use of a $750 \mathrm{ml}$ spacer device and their BDP dose left unchanged. Four patients were taking $1500 \mu \mathrm{g}$, five $2000 \mu \mathrm{g}$ and one $2500 \mu \mathrm{g}$ BDP daily. HPA function tests were repeated nine days to eight weeks later. Patients took their usual morning dose of BDP prior to all blood tests. Both $9 \mathrm{am}$ serum cortisol and $24 \mathrm{~h}$ urine free cortisol increased with use of the spacer $(p<$ 0.01 , Wilcoxon signed rank test). The rise in serum cortisol in response to tetracosactrin did not change significantly. In six patients the HPA assessment while they were using the spacer was normal. The greatest improvement in HPA function occurred in those who had not previously required long term prednisolone. We conclude that large volume spacer devices may reduce the HPA suppression associated with high doses of beclomethasone dipropionate.

Role of intravenous aminophylline in the management of acute severe asthma

MS CONNELLY, PF JENKINS, BDW HARRISON West Norwich Hospital, Norwich A double blind placebo controlled trial was undertaken to assess the value of intravenous aminophylline in the management of acute severe asthma. All patients were treated with regular nebulised bronchodilators (terbutaline $5 \mathrm{mg}$ four hourly) and oral steroids ( $40 \mathrm{mg}$ daily) or, if vomiting, intravenous steroids $(3 \mathrm{mg} / \mathrm{kg}$ IV followed by $0.5 \mathrm{mg} / \mathrm{kg} / \mathrm{h}$ ). Patients were then randomised to receive either intravenous aminophylline $(1 \mathrm{mg} / \mathrm{kg} / \mathrm{h})$ or normal saline. The intention was to study 50 patients, half receiving aminophylline and half receiving placebo. However, the study was discontinued because of the results obtained. Seven patients received placebo and five were withdrawn from the study within six hours: in three their condition failed to improve objectively or actually worsened and a fourth required ventilation; in the two patients who were not withdrawn objective improvement was only slight. Of the 10 patients receiving aminophylline treatment all improved objectively during the first six hours, five showing increases of peak flow of $30 \%$ or more, and none was withdrawn. In view of these findings we felt it unethical to continue with the study. These results support the view that most patients with severe asthma benefit from the addition of intravenous aminophylline to standard treatment with steroids and nebulised beta agonist.

Peak flow measurement in general practice: comparison of the miniWright peak flow meter and the turbine spirometer

KEVIN JONES, MARK MULLEE Faculty of Medicine, University of Southampton The turbine spirometer is a relatively new instrument which provides not only FEV, and FVC measurements, but also PEF and percentages of predicted values. Its cost makes it potentially useful in general practice and so this study has compared peak expiratory flow recordings made using this machine and the

\begin{tabular}{|c|c|c|c|c|}
\hline \multirow{3}{*}{$\begin{array}{l}\text { Salb prep } \\
\text { (at } 8 l / \text { min) }\end{array}$} & \multicolumn{2}{|c|}{ Laboratory results $(n=5)$} & \multirow{3}{*}{$\begin{array}{l}\begin{array}{l}\% \text { Prep } \\
\text { left }\end{array} \\
22 \cdot 0 \\
14 \cdot 8 \\
28 \cdot 8 \\
50.9\end{array}$} & \multirow{3}{*}{$\begin{array}{l}\text { Time for } \\
\text { neb (min) }\end{array}$} \\
\hline & Original wt (mg) & Wt after neb (mg) & & \\
\hline & $\begin{array}{ll}5.05 & (0.02) \\
5.34 & (0.04) \\
5.26 & (0.05) \\
2.68 & (0.03)\end{array}$ & $\begin{array}{ll}1.19 & (0.04) \\
0.82 & (0.11) \\
1.50 & (0.20) \\
1.39 & (0.02)\end{array}$ & & \\
\hline $\begin{array}{l}\text { Resp soln } \\
0 \cdot 2 \% \text { no BKC }\end{array}$ & $\begin{array}{l}\text { Patient results (n } \\
4.75 \quad(0.26) \\
5.02 \quad(0.27)\end{array}$ & $\begin{array}{ll}0) & \\
0.91 & (0.40)^{\star} \\
1.74 & (0.47)^{\star}\end{array}$ & $\begin{array}{l}19 \cdot 1 \\
35 \cdot 3\end{array}$ & $\begin{array}{l}15 \cdot 9 \\
11 \cdot 3\end{array}$ \\
\hline
\end{tabular}

${ }^{\star} \mathrm{p}<0.001$

more commonly used mini-Wright peak flow meter. Measurements of peak flow rates were taken on patients with asthma or obstructive airways disease during routine surgeries. The patient's age and sex were recorded, and the order of use of the two instruments was randomly decided. The readings obtained on the mini-Wright meter (Wright) were usually higher than those on the turbine spirometer (Turbine). Therefore the differences between the readings of the two meters were calculated as follows: Wright minus Turbine. The differences followed a normal distribution and so a paired $t$ test was performed to compare 192 pairs of observations. The mean difference was 87.77 with a standard deviation of 43.87 ( $p<0.001)$. Thus there is a marked and highly significant difference between the PEF readings obtained on the two instruments. When the percentage differences between the readings of the two meters were calculated the range of differences was found to be from $33 \%$ to $39 \%$. The order of use of the two instruments and the type of blow performed (PEF type or FVC type) were not found to be significant in either actual values or percentage differences. The two instruments clearly do not produce peak flow readings which are comparable. Considerable variation suggests that comparisons are likely to be misleading for the patient. The mini-Wright peak flow meter is likely therefore to remain the preferred instrument for the measurement of peak flow in general practice.

Effect on nebulisation of removing the preservative from salbutamol nebuliser solutions

DR GRAHAM, SD KING, C JACKSON, CRK HIND Regional Adult Cardiothoracic Unit and Pharmacy, Broadgreen Hospital, Liverpool The original salbutamol nebuliser solution, Ventolin respirator solution, contained the preservative benzalkonium chloride (BKC), which has surfactant like properties. Because BKC may cause bronchoconstriction, there has been a switch to preservative free nebules $(0.1 \%: 5 \mathrm{mg}$ in $5 \mathrm{ml}$, or $0.2 \%: 5 \mathrm{mg}$ in 2.5 $\mathrm{ml})$. However, some patients have reported decreased effectiveness of the BKC free $0.2 \%$ nebules. A possible explanation for this is that a greater percentage of the intended dose of salbutamol remains in the nebuliser apparatus. To study this further, we have per- formed two experiments to estimate the amounts of salbutamol delivered with each formulation. Four different preparations ( $5 \mathrm{mg}$ salbutamol in each) were nebulised under the same conditions (at 5 and $81 / \mathrm{min}$ ) in the laboratory using both the Wright and the Acorn nebuliser. Each nebuliser and tubing were weighed dry, when loaded with solution, and then after nebulisation had finished. The experiment was then repeated using patients (at $8 \mathrm{l} / \mathrm{min}$ ). These results (table above) suggest that removal of the preservative (BKC) and reduction in volume of salbutamol nebuliser solutions significantly increases the proportion remaining within the nebuliser apparatus. Such an effect may reduce the efficacy of the nebulisation.

Trial of daily peak expiratory flow rate monitoring in the management of asthma

JP JAMISON, RK MCKINLEY, WG IRWIN Departments of Physiology and General Practice, Queen's University, Belfast With ethical committee approval, asthmatic patients selected from general practitioner lists were shown how to measure their own peak expiratory flow rate (PEF) and requested to monitor their PEF three times daily, taking the best of three readings on each occasion. All patients had a preliminary two week period of monitoring. The maximum within day difference in PEF expressed as a percentage of the minimum PEF on that day in 12 consecutive days of monitoring (\% variability), and the overall mean PEF for the same period were computed ( $J$ Physiol 1989 (in press)). The patients were assigned randomly to either monitor or not to monitor their PEF for the next six months. Those who continued monitoring were taught self management responses to low PEF readings. The measurements of $\%$ variability and mean PEF were then repeated over a further 12 day period in both groups. There was a highly significant improvement in \% variability in both groups after six months ( $p<0.001$, analysis of variance) but no significant difference with six months' monitoring (table below). Mean PEF showed no significant change. By these PEF criteria, it appears that there was no additional benefit from prolonged routine monitoring after the first two weeks. The variability of PEF may be a more sensitive index than mean PEF in the assessment of asthma.

\begin{tabular}{|c|c|c|c|c|c|}
\hline & \multicolumn{5}{|c|}{$\begin{array}{l}\text { Geometric mean of the maximum within-day \% variability in } P E F \text { and mean } \\
P E F \text { over } 12 \text { days, before and after six months, with and without monitoring }\end{array}$} \\
\hline & \multirow[b]{2}{*}{$n$} & \multicolumn{2}{|c|}{$\%$ variability } & \multicolumn{2}{|c|}{ Mean PEF $\left(l s^{-t}\right)$} \\
\hline & & Before & 6 mon & Before & 6 mon \\
\hline $\begin{array}{l}\text { Without monitoring } \\
\text { With monitoring }\end{array}$ & $\begin{array}{l}46 \\
32\end{array}$ & $\begin{array}{l}45 \\
37\end{array}$ & $\begin{array}{l}32 \\
26\end{array}$ & $\begin{array}{l}5 \cdot 7 \\
5 \cdot 4\end{array}$ & $\begin{array}{l}5 \cdot 8 \\
5 \cdot 8\end{array}$ \\
\hline
\end{tabular}


Use of bronchodilators in conjunction with evidential breath alcohol testing devices by patients with respiratory diseases

N MCI JOHNSON, K UPTON, PJ GOMM, CG BROSTER, MD OSSELTON Central Research and Support Establishment, Home Office Forensic Science Service, Aldermaston, Reading, and University College and Middlesex School of Medicine, and Whittington Hospitals, London Patients with respiratory diseases who experience difficulty in providing adequate breath samples for evidential breath alcoho testing devices have on occasions had to resort to the use of bronchodilators. Previous work has shown that the contents of respiratory aerosol inhalers containing bronchodilators and nasal sprays gave no apparent alcohol reading when used in conjunction with any of the breath alcohol testing devices approved for police use in Great Britain. Experiments were performed to assess what effects, if any, the use of bronchodilators would have on the pulmonary function test results, and breath alcoho test results of patients suffering from respiratory diseases who had consumed alcohol Ten patients were selected from those attending the chest clinics at the Middlesex and Whittington Hospitals. The subjects were divided into small groups and included a control subject for the duration of the testing schedule. Pulmonary function tests and evidential breath alcohol readings were conducted at the start of the testing schedule and after the consumption of measured quantities of an alcoholic beverage. Further tests were then carried out following the use of a bronchodilator and at timed intervals up to one hour after its use. Blood samples were also taken during the experiment so that an assessment could be made of any effect that the use of a bronchodilator would have on the blood: breath ratio of alcohol in the body. The results showed that, although the use of a bronchodilator generally improved the subject's ability to perform the pulmonary function tests, it did not have any effect on the results from the evidential breath alcohol testing device. The blood:breath ratios obtained from the subjects tested were within the normal range expected.

ECG changes with inhaled salbutamo following treatment with bendrofluazide and the effects of replacement with potassium, magnesium, and triamterene

BJ LIPWORTH, DG McDEVITT, RA CLARK, EG SERVICE, AD STRUTHERS Departments of Respiratory Medicine and Clinical Pharmacology, Ninewells Hospital, Dundee Beta agonists and diuretics are often prescribed together in patients with airflow obstruction, and both cause hypokalaemia. This in turn may predispose to the development of ventricular arrhythmias, particularly in patients with coexistent ischaemic heart disease (Stewart $e$ al. Br Heart J 1985;54:290). We studied 12 healthy volunteers ( 28 (SD 2) y), who were given five days' treatment with placebo, bendrofluazide $5 \mathrm{mg}$ (B), B + potassium 24 mmol (K), B + magnesium $24 \mathrm{mmol}(\mathrm{Mg})$ $\mathrm{B}+\mathrm{K}+\mathrm{Mg}$, and $\mathrm{B}+$ triamterene $50 \mathrm{mg}$. After each treatment subjects were given two doses (each of $1 \mathrm{mg}$ ) of inhaled salbutamol (S). ECG parameters and plasma $\mathrm{K}$ and $\mathbf{M g}$ were measured 25 minutes after each dose.
Treatment with $\mathrm{B}+\mathrm{S}$ produced a lower absolute level of potassium than $\mathrm{S}$ alone (means and 95\% CI): 2.91 (2.65-3.16) $\mathrm{mmol} / 1$ v $3.38(3.12-3.64) \mathrm{mmol} / \mathrm{l}$ (p < $0.001)$. The addition of $\mathrm{K}, \mathrm{Mg}$ or triamterene did not attenuate this effect. Plasma magnesium was unchanged. Salbutamol produced $T$ wave flattening ( $p<0.001$ ), Q-T prolongation ( $p<0.001$ ), and in some subjects $U$ waves and $S-T$ segment depression. Pretreatment with B potentiated Q-T prolongation due to salbutamol $(p<0.001)$, but only augmented $T$ wave flattening in six cases $(p<0.002)$. The addition of triamterene attenuated the $T$ wave response to $B+S$ in five subjects ( $p<0.01$ ). Bendrofluazide also increased the frequency of salbutamol induced $U$ waves, and this was attenuated by triamterene in some subjects. Supplementation with $\mathrm{K}$ and $\mathrm{Mg}$ had no effect of ECG changes. Thus the hypokalaemic and ECG sequelae of inhaled salbutamol were potentiated by diuretic, and were unaffected by supplementation with $\mathrm{K}$ and $\mathrm{Mg}$. The addition of triamterene attenuated ECG effects, but only in some subjects. These changes may predispose to cardiac arrhythmias in susceptible patients, particularly during acute episodes of airflow obstruction, when hypoxaemia and adrenergic drive may further sensitise the myocardium.

Peak flow responses to home nebuliser treatment

BRC O'DRISCOLL, RJ TAYLOR, EA KAY, H WEATHERBY, MCP CHETTY, A BERNSTEIN Hope Hospital, Salford Fifty patients (16 asthma, mean $\mathrm{FEV}_{1} / \mathrm{FVC} 1 \cdot 3 / 2 \cdot 1 \quad$ 1; 34 COPD, mean FEV,/FVC $0.7 / 1.8$ ) were referred for consideration of home nebuliser treatment. All were monitored for two weeks while using their usual inhaled treatment followed by two weeks using a "Nebuhaler" spacer to deliver $1 \mathrm{mg}$ of terbutaline and 80 $\mu \mathrm{g}$ of ipratropium bromide (IB) four times daily. They then borrowed a System 22 nebuliser to self administer salbutamol (S) nebuliser solution ( $5 \mathrm{mg}$ ), IB unit dose vials $(0.5 \mathrm{mg})$ or a mixture of these drugs four times daily for one month each. We have previously reported that 33 of these patients derived long term subjective benefit from home nebuliser treatment (Thorax 1989; 44:845P). Their mean daily PEF recordings are shown in the table. Of 33 patients who chose home nebuliser therapy for long term use, 25 (including 15 COPD patients) had their highest mean daily PEF while using a home nebuliser. Six of seven patients who preferred Nebuhaler to nebuliser treatment had their highest PEF during Nebuhaler treatment. We conclude that domiciliary trials will identify optimal bronchodilator treatment for individual patients with severe airflow obstruction. Many such patients will derive subjective and objective benefit from home nebuliser treatment.

\begin{tabular}{|c|c|c|c|c|c|}
\hline & $\begin{array}{l}\text { Usual } \\
\text { treatment }\end{array}$ & Nebuhaler & $S N e b$ & $I B N e b$ & Mix Neb \\
\hline $\begin{array}{l}\text { Asthma } \\
\text { COPD }\end{array}$ & $\begin{array}{l}205 \\
168\end{array}$ & $\begin{array}{l}215 \\
186\end{array}$ & $\begin{array}{l}246 \\
180\end{array}$ & $\begin{array}{l}244 \\
178\end{array}$ & $\begin{array}{l}274 \\
196\end{array}$ \\
\hline \multicolumn{6}{|c|}{ Statistical analysis-Wilcoxon matched pairs, $p$ values } \\
\hline $\begin{array}{l}\text { Nebuhaler } \\
\text { Salb nebuliser } \\
\text { IB nebuliser } \\
\text { Mixed nebuliser } \\
\text { Mixed nebuliser }\end{array}$ & $\begin{array}{l}>\text { usual treatment } \\
>\text { usual treatment } \\
>\text { usual treatment } \\
>\text { usual treatment } \\
>\text { Nebuhaler }\end{array}$ & & \multicolumn{2}{|c|}{$\begin{array}{l}0.07 \\
0.02 \\
0.07 \\
0.03 \\
0.03\end{array}$} & $\begin{array}{l}0.0004 \\
0.03 \\
0.02 \\
0.01 \\
\text { NS }\end{array}$ \\
\hline
\end{tabular}

Dermal thinning and purpura associated with high dose inhaled corticosteroids

S REYNOLDS, S CAPEWELL, D SHUTTLEWORTH, C EDWARDS, AY FINLAY Departments of Chest Diseases and Dermatology, University of Wales College of Medicine, Llandough Hospital, Cardiff High dose inhaled corticosteroid therapy for asthma can cause a variety of systemic effects (Stead and Cooke. $\mathrm{Br}$ Med J 1989;298:403). In order to assess potential effects on the skin, we studied 68 outpatients with asthma or chronic bronchitis, grouped according to their corticosteroid therapy and matched for age and sex. Skin thickness, measured by pulsed A-scan ultrasound, was reduced by $31 \%$ ( $95 \%$ confidence interval $19-43 \%, p<0.001)$ in 15 patients on long term prednisolone compared with 17 control patients. Skin thickness was reduced by $14 \%$ (CI $2.5-25.5 \%, \mathrm{p}<0.05)$ in 21 patients taking long term high dose inhaled corticosteroids, but was unchanged in 15 patients on low dose inhaled corticosteroids. The incidence of purpura was also increased in patients on prednisolone $(80 \%, p<0.001)$ and in those on high dose inhaled corticosteroids $(48 \% v 12 \%$ in controls, $p<0.05$ ). This skin thinning and purpura represents further evidence of systemic effects from high dose inhaled corticosteroids.

Asthmalog: communication between patient and doctor

AR TANSER, SR YARDE, H GIMBLETT, PA JONES, CMB HIG Royal United Hospital, Bath We have not been satisfied with commercially available material designed to aid communication with asthmatic patients. We will demonstrate a system based on a $180 \times 105 \mathrm{~mm}$ notebook, containing pages of information relevant to the individual. The pages are printed with a desktop publishing program and a laser printer. With an 8 point Swiss font, 300 words can be printed on each side with good legibility. Charts for peak flow records and for medication details are folded twice and measure $155 \times 280 \mathrm{~mm}$. The Asthmalog contains general information, details of drugs, and information about gauging the severity of asthma. On a yellow page there are instructions for emergency treatment for attending doctors which contain warnings about overuse of xanthines if oral medication has been prescribed. Patients are encouraged to write comments and record events on diary pages. Forty five out of 63 patients returned a questionnaire. Forty four were enthusiastic and one had not read it. Thirty seven had shown their Asthmalog to friends or relatives and 27 to their GP or nurse. As a result of advice from patients or others, we are easily able to change the content, and the pages can be updated regularly and reissued. We plan to encourage

.


general practitioners and nurses to use the book as a method of communication both with the patient and with the hospital.

Reversibility testing in COAD: poor correlation with response to a home nebuliser

C TEALE, JFJ MORRISON, PC JONES, MF MUERS Respiratory Unit, Killingbeck Hospital, Leeds We previously reported the effects of home nebuliser therapy in non-steroid responsive COAD (BTS Proceedings. Thorax $1989 ; 44: 339 \mathrm{P})$. We now describe a further analysis of these patients responses to determine the reproducibility of their reversibility tests and any correlation between short term reversibility and response to a home nebuliser. Twenty patients with severe COAD (mean age 66, mean $\mathrm{FEV}_{1} 0.87 \mathrm{l}$ ) and little reversibility ( $<20 \%$ increase in $\mathrm{FEV}_{1}$ after two puffs salbutamol or fenoterol via MDI and $<25 \%$ increase in PEF on oral steroids recorded home PEF throughout the study. During a run in patients continued normal medication and performed three reversibility tests at weekly intervals. Spirometry, lung volumes and sGaw were recorded before and after a mixture of nebulised ipratropium 0.5 $\mathrm{mg}(\mathrm{I})$ and fenoterol $1.25 \mathrm{mg}(\mathrm{F})$. Patients then received nebulised $I+F$ or saline placebo $(P)$ for three week blocks in a double blind crossover study. Repeat reversibility tests (PEF, FEV, FVC) were poorly reproducible ( $p>0.05$ ). Mean PEF on home nebuliser rose from $164(\mathrm{P})$ to $196(\mathrm{I}+\mathrm{F})$, $p=0.0001$. Reversibility of sGaw correlated with improvement in home PEF on nebulise (mean PEF $(\mathrm{I}+\mathrm{F})$ - mean PEF (P)) $r=0.82, p<0.001 ;$ correlations for all other indices were not significant. We conclude reversibility tests are poorly reproducible in patients with severe COAD and, excepting sGaw, do not correlate with response to regular nebuliser therapy. Home measurements of PEF may be the most suitable objective way of assessing suitability for and response to a home nebuliser in patients with COAD.

Inspiratory flow responses to exercise and terbutaline in asthmatic children

PC SEDDON, PI MACFARLANE, DP HEAF Alder Hey Children's Hospital, Liverpoo Recurrent stridor in childhood ("allergic croup") is associated with atopy and asthma. To investigate whether upper airway obstruction occurred more widely among childhood asthmatics, maximal flow-volume loops were recorded in response to exercise, in asthmatic and normal children aged 7-14 years, and inhaled terbutaline (asthmatics only). Peak inspiratory flow (PIF), maximum inspiratory flow at $50 \%$ of vital capacity $\left(I_{50}\right)$, and ratio of maximum expiratory to inspiratory flow at $50 \%$ VC $\left(\mathbf{R}_{50}\right)$ were measured from the curves, and changes in these indices were compared in 10 asthmatics who showed exercise induced bronchoconstriction (EIB: $\geqslant 15 \%$ fall in $\mathrm{FEV}_{1}$ ), 10 asthmatics who did not show EIB, and 10 normal children with no atopic family history. The results are shown in the table. During exercise all three groups had small increases in PIF and $I_{50}$. After exercise the asthmatics with EIB had a sustained fall in PIF and $I_{50}$, which improved after terbutaline. $R_{50}$ was unchanged during exercise but increased in both asthmatic groups after bronchodilator. Our results suggest that extrathoracic airway narrowing may occur during exercise in asthmatic children.

\begin{tabular}{llll}
\hline $\begin{array}{l}\text { Mean \% } \\
\text { change from } \\
\text { baseline }\end{array}$ & $\begin{array}{l}\text { During } \\
\text { exercise }\end{array}$ & $\begin{array}{l}9 \mathrm{~min} \\
\text { after } \\
\text { exercise }\end{array}$ & $\begin{array}{l}10 \mathrm{~min} \\
\text { after } \\
\text { terbutaline }\end{array}$ \\
\hline $\mathrm{I}_{50}$ & & & \\
Asthma +EIB & +6.4 & -17.7 & +13.4 \\
Asthma -EIB & +9.7 & -3.9 & +2.1 \\
Normal & +6.6 & -2.6 & \\
& & & \\
$\mathrm{R}_{\text {s0 }}$ & & & \\
Asthma +EIB & +5.8 & +15.9 & +43.4 \\
Asthma-EIB & -3.5 & -4.8 & +28.3 \\
Normal & -4.4 & -3.2 & \\
\hline
\end{tabular}

Use of a management plan for the treatment of asthma in an emergency department

I TOWN, T KWONG, $P$ HOLST, $R$ BEASLEY Department of Medicine, Wellington School of Medicine, Wellington, New Zealand We have investigated the effect of a standardised management protocol on the medical assessment and treatment of adult asthmatic patients attending the Wellington Hospital emergency department. The management protocol consisted of an assessment sheet for the recording of the essential features of the history and examination and a treatment flow sheet with guidelines for initial management, which were based on the objective assessment of airflow obstruction. The study was based on the retrospective analysis of emergency department records before and one year after the introduction of the protocol. We observed that during both study periods the emergency department accepted a primary care role for the treatment of acute asthma, most patients $\left(90^{\circ}{ }_{0}\right)$ being self referred. The use of a standardised assessment sheet prompted enquiry about high risk features such as previous life threatening episodes. Although objective measurement of airflow obstruction was regularly performed during both periods $\left(84^{\circ}{ }_{0}\right.$ in $1986 v 88^{\circ}$ in 1987$)$ the introduction of the protocol, which was based on FEV recordings, resulted in a shift to the use of the spirometer and encouraged a greater use of serial measurements to assess the response to treatment $\left(69^{\circ}\right.$ in $1986 v 85^{\circ}$ in $1987, \mathrm{p}<$ $0.01)$. The institution of specific guidelines for management resulted in a change in the emphasis of treatment, with an increased use of intravenous corticosteroids $(36 \%$ in $1986 v$ $61^{\circ}{ }_{0}$ in 1987, $p<0.01$ ) and the use of a second dose of nebulised bronchodilator $\left(25^{\circ}{ }_{0}\right.$ in $1986 v 60^{\circ}$ o in $\left.1987, \mathrm{p}<0.01\right)$. In 1987 a greater proportion of patients discharged from the emergency department were prescribed a course of oral corticosteroids and had follow up arrangements documented. We conclude that the implementation of a protocol, in which guidelines for treatment are based on the objective assessment of airflow obstruction, results in management which is in line with current recommendations for the treatment of acute asthma.

\section{Airways effects of inhaled chlorbutol in} asthmatic subjects

H WINDOM, C BURGESS, J CRANE, R BEASLEY Department of Medicine, Wellington School of Medicine, Wellington, New
Zealand The presence of preservatives in bronchodilator nebuliser solutions may influence the magnitude of the airways response. Problems have been identified with sulphites, benzalkonium chloride and EDTA, agents which can cause bronchoconstriction when inhaled by asthmatic subjects. One preservative whose airways effects are unknown is chlorbutol, the antibacterial and antifungal agent which is incorporated in terbutaline nebuliser solution in a concentration of $5 \mathrm{mg} / \mathrm{ml}$. In this study we have undertaken bronchial provocation testing with chlorbutol to determine its airways effects. Ten stable atopic asthmatic subjects (mean age 25 years, baseline FEV, $95 \%$ predicted) attended the laboratory on two occasions to undertake bronchial challenge testing with either methacholine or chlorbutol according to a double blind protocol. Subjects inhaled doubling concentrations of methacholine $(0 \cdot 13-4.0 \mathrm{mg} / \mathrm{ml})$ or chlorbutol $(0 \cdot 16-5.0 \mathrm{mg} / \mathrm{ml})$ through a face mask, during tidal breathing, for two minutes. Increasing concentrations of each solution was inhaled until the FEV , had fallen by $>20 \%$, or the final concentration was administered. The nine subjects completing the study had a geometric mean $\mathrm{PC}_{20}$ methacholine of $0.24 \mathrm{mg} / \mathrm{ml}$ (range $<0.13-0.50$ ). One subject experienced a fall in $\mathrm{FEV}_{1}>$ $20 \%$ after inhalation of $2.5 \mathrm{mg} / \mathrm{ml}$ chlorbutol. In the remaining eight subjects inhalation of chlorbutol did not affect airway calibre, with a mean change in $\mathrm{FEV}_{1}$ of $+3 \%$ after inhalation of the $5 \mathrm{mg} / \mathrm{ml}$ solution. We conclude that chlorbutol, in the dose present in terbutaline nebuliser solution, has no significant effect on airway calibre when inhaled by most asthmatic subjects. Although bronchoconstriction may occur with chlorbutol in some asthmatic subjects, it remains to be determined whether this would affect the overall airways response when inhaled in combination with a potent beta adrenergic $^{2}$ agonist such as terbutaline.

Self administration of inhaled beta agonist drugs during severe asthma

H WINDOM, C BURGESS, J CRANE, N PEARCE, I KWONG, R BEASLEY Departments of Medicine and Community Health, Wellington School of Medicine, Wellington, New Zealand Interviews were conducted with 99 consecutive adult patients admitted to Wellington Hospital with a diagnosis of asthma in order to assess the extent to which beta agonist drugs are self administered by asthmatic patients during severe asthma. The mean admission $\mathrm{FEV}_{1}$ was $29 \%$ predicted. Regular prescribed medication included inhaled beta agonists in $100 \%$, inhaled steroids $76 \%$, oral steroids $18 \%$, oral theophylline $40 \%$, oral beta agonist $13 \%$, and a short course of oral steroids was prescribed in $19 \%$ of patients. Patients were subdivided into two groups: group A comprising 79 patients prescribed a beta agonist for inhalation via a metered dose inhaler (MDI) alone and group B comprising 20 patients prescribed beta agonist for inhalation via both an MDI and nebuliser. In group A the attacks of asthma lasted $>24$ hours in $64 / 79$ patients, and $22 \%$ of these patients reported taking more than 60 puffs and $51 \%$ more than 30 puffs of their MDI during the 24 hour period prior to admission. In group B the attacks of asthma lasted $>24$ hours in 17/ 20 patients, and $35 \%$ of these patients self administered their nebuliser more than six 
times, and $76 \%$ more than four times during the 24 hour period prior to admission. In addition to their nebuliser use, these patients also took a median 22 puffs of their MDI during this 24 hour period. This use of inhaled beta agonist contrasts with the recommended practice in both the USA (Hodgkin JE. Chest 1986;90(suppl 5):62) and Europe (Vermeire PA, et al. Chest 1986;90(suppl 5):58), where most physicians recommend no more than 15 puffs of a beta agonist as the maximal dose per day. We conclude that asthmatic patients in New Zealand self administer high doses of inhaled beta $_{2}$ agonist drugs during severe exacerbations of asthma.

\section{Inhaled salbutamol in COAD: wide range of optimal doses}

C TEALE, JFJ MORRISON, SB PEARSON Respiratory Unit, Killingbeck Hospital, Leeds Patients with COAD are often treated with increasing doses of bronchodilators although this raises concerns over side effects and possible development of tolerance. In an open study we have determined the range of doses required to produce at least $90 \%$ bronchodilatation in patients with COAD. We studied 26 patients with stable COAD, mean age 54 years (range 31-82), and mean $\mathrm{FEV}_{1}$ 1.9 (SEM 0.21) 1 , all of whom showed $<20 \%$ improvement in FEV, following salbutamol (S) $0.2 \mathrm{mg}$ by metered dose inhaler (MDI). Inhaled bronchodilators were omitted 12

\begin{tabular}{llllllll}
\hline & \multicolumn{8}{c}{ Minimal dose $(\mathrm{mg})$ of salbutamol to produce } & $90 \%$ bronchodilatation $(n=26)$ \\
\cline { 2 - 8 } & 0.2 & 0.4 & 0.6 & 0.8 & 1.0 & $1 \cdot 2$ & $>1 \cdot 2$ \\
\hline No (\%) of subjects & 3 & 6 & 8 & 3 & 2 & 2 & 3 \\
& $(12)$ & $(23)$ & $(31)$ & $(12)$ & $(8)$ & $(8)$ & $(12)$ \\
\hline
\end{tabular}

hours before each study. Baseline $\mathrm{FEV}_{1}$ was recorded (best of nine blows over one hour) Subjects then took $S 0.2 \mathrm{mg}$ by $\mathrm{MDI}$ and $\mathrm{FEV}_{1}$ was measured 15 minutes later. The procedure was repeated until a cumulative dose of $1.2 \mathrm{mg}$ had been given. Responses were analysed by linear regression to calculate the dose of $\mathrm{S}$ to produce $90 \%$ of maximal bronchodilatation. These results suggest that in COAD there is wide individual variation in the dose of $S$ required to produce $90 \%$ bronchodilatation, most patients $(58 \%)$ achieving this level with only $0.6 \mathrm{mg} \mathrm{S}$ and few $(12 \%)$ requiring more than $1.2 \mathrm{mg}$. This supports a trial of high dose MDI bronchodilator therapy before a home nebuliser in COAD.

Regular nebulised terbutaline in COAD: baseline improves without tolerance

C TEALE, SB PEARSON Respiratory Unit, Killingbeck Hospital, Leeds There is uncertainty over the long term effects of high dose nebulised bronchodilator therapy in COAD and conflicting evidence on the possible development of tolerance to beta, agonists. This study addresses these problems by examining dose responses in 10 subjects with $\mathrm{COAD}$ before and after starting regular nebulised terbutaline (T). We studied 10 subjects with severe $\mathrm{COAD}$, mean age 63 years and mean $\mathrm{FEV}_{1} 0.81$. We measured cumulative dose-response curves for six incremental doses of $\mathrm{T}(0.5-8 \mathrm{mg})$ given by Turbohaler. Dose responses were repeated 1 , 4, 8 and 12 weeks after starting nebulised $T 5$ mg qds. Other medication remained constant; inhaled bronchodilators were omitted at least six hours before each study. Maximal bronchodilatation (Emax) was calculated by linear regression and the results were analysed by paired $t$ test. Mean baseline $F_{1}, F V C$ and $P E F$ all rose after one week of nebulised $T(p<0.05)$-for example, PEF rose from 142 to $170 \mathrm{l} / \mathrm{min}$, with non-significant trends to further improvement up to two months with nebulised T. Baseline TLC fell from 7.7 to 7.31 (p $<0.05$ ) on nebulised T. Initial mean Emax PEF was 208 1/min, with T $8 \mathrm{mg}$ producing a mean bronchodilatation of $100 \cdot 2 \%$ of Emax; there was no significant change in either parameter after starting nebulised $T$. We conclude that regular nebulised $T 5 \mathrm{mg}$ qds improves baseline lung function in severe COAD as seen by improvements in $\mathrm{FEV}_{1}, \mathrm{FVC}, \mathrm{PEF}$ and TLC. Neither Emax nor \% Emax achieved by $\mathrm{T} 8 \mathrm{mg}$ changed after the start of regular $T$ by nebuliser, implying that tolerance did not develop. 